REINFORCING MECHANISMS OF

SILICA / SULFIDE-SILANE VS. MERCAPTO-SILANE FILLED

TIRE TREAD COMPOUNDS 
YOKOHAMA This research is a joint project between the University of Twente and The Yokohama Rubber Co., Ltd.

\section{Graduation committee}

\begin{tabular}{|c|c|c|}
\hline Chairman: & Prof. Dr. G.P.M.R. Dewulf & University of Twente, ET \\
\hline Secretary: & Prof. Dr. G.P.M.R. Dewulf & \\
\hline Supervisor: & Prof. Dr. A. Blume & University of Twente, ET \\
\hline \multirow[t]{5}{*}{ Members: } & Prof. Dr. A.J.A. Winnubst & $\begin{array}{l}\text { University of Twente, ET / University of } \\
\text { Science and Technology of China }\end{array}$ \\
\hline & Dr. M.A. Hempenius & University of Twente, TNW \\
\hline & Prof. Dr. U. Giese & Deutsches Institut für \\
\hline & & Kautschuktechnologie e. V., Germany \\
\hline & Prof. Dr. M.S. Galimberti & Politecnico di Milano, Italy \\
\hline Expert: & Mr. A. Hasse & $\begin{array}{l}\text { Evonik Resource Efficiency GmbH, } \\
\text { Germany }\end{array}$ \\
\hline Referee: & Dr. N. Amino & The Yokohama Rubber Co., Ltd., Japan \\
\hline
\end{tabular}

Reinforcing mechanisms of silica / sulfide-silane vs. mercapto-silane filled tire tread compounds

By Masaki Sato

Ph.D. Thesis, University of Twente, Enschede, the Netherlands

With references - With summary in English and Dutch.

Copy right (C) Masaki Sato, 2018.

All rights reserved.

Printed at IPSKAMP printing, Auke Vleerstraat 145, 7547 PH Enschede, the Netherlands.

ISBN: 978-90-365-4593-8

DOI: $10.3990 / 1.9789036545938$

USL: https://doi.org/10.3990/1.9789036545938 


\title{
REINFORCING MECHANISMS OF SILICA / SULFIDE-SILANE VS. MERCAPTO-SILANE FILLED TIRE TREAD COMPOUNDS
}

\author{
DISSERTATION
}

\author{
to obtain \\ the degree of doctor at the University of Twente, \\ on the authority of the rector magnificus, \\ prof.dr. T.T.M. Palstra, \\ on account of the decision of the graduation committee, \\ to be publicly defended \\ on Wednesday, the $18^{\text {th }}$ of July, 2018 at $14: 45$ hours
}

by

Masaki Sato

born on the $7^{\text {th }}$ of October, 1981

in Miyagi, Japan 
This dissertation has been approved by:

Supervisor: $\quad$ Prof. Dr. A. Blume 


\section{Table of contents}

Chapter 1: Introduction

Chapter 2: Literature survey;

Overview of reinforcing mechanism of silica filled rubber and silane chemistry

Chapter 3: Model compound study-1;

Reactivity study of mercapto-silane and sulfide-silane with various types of olefin

Chapter 4: $\quad$ Model compound study-2;

Influence of silica, $\mathrm{ZnO}, \mathrm{CBS}$ and sulfur on the reaction between mercapto-silane and model olefin

Chapter 5: Model compound study-3;

Reactivity study of mercapto-silane and sulfide-silane with squalene or liquid butadiene

Chapter 6: Rubber compound study-1;

Working mechanism of mercapto-silane or sulfide-silane in silica filled SBR/BR compounds

Chapter 7: Rubber compound study-2;

Comparison of silica filled NR or IR compounds with silica filled SBR/BR compounds in both silane systems

Chapter 8: Summary

Samenvatting 



\section{Chapter 1}

\section{Introduction}

\subsection{Introduction}

Although a wide variety of performances are required for a tire, the significance of a low rolling resistance tire has increased over the years because of an increased consciousness to aim for an eco-friendly society. Low rolling resistance tires can lead to the reduction of fuel consumption resulting in the contribution of a preservation of petroleum resources, and even important to a reduction of the $\mathrm{CO}_{2}$ emission.

It is necessary to reduce the energy dissipation of rubbers in the tire components during deformation for the improvement of the rolling resistance level. The energy dissipation of the rubber correlates with the loss tangent $(\tan \delta)$ of the rubber. However, there is a certain risk that a reduction of the loss tangent of the rubber causes a reduction of the grip performance which is another very important property of a tire. Three properties, which are rolling resistance, wet grip performance and also abrasion resistance form the "magic triangle", which means that it is very difficult to shift all three properties at the same time to a high level. There are many trade-offs between these three properties. In addition to the above mentioned relationship between wet grip and rolling resistance, for instance, the decrease of the $\mathrm{Tg}$ of the compound is also a possibility to improve the abrasion resistance, however this leads directly to a loss in the wet grip performance.

One of the biggest technical innovations to expand the magic triangle was the "Green Tire Technology", which was introduced by Michelin in 1992. In this technology, they introduced a highly dispersible silica as a reinforcing filler instead of carbon black together with a silane coupling agent in a solution SBR/ BR blend. This technology enables to improve wet grip performance and rolling resistance at the same time without having a negative effect on the abrasion resistance. The challenge was: Silica has a polar functionality on the surface, but those rubbers which are normally used for tires are non-polar, olefinic hydrocarbon materials. Therefore, the hydrophobation of the silica surface is one of the key 
factors to enable the silica clusters to be well dispersed inside the rubber matrix. Furthermore, to gain a sufficient reinforcing effect by silica, an interaction between the rubber and silica has to be established. Bi-functional silane coupling agents, containing one side which can react with the silica surface and the other side which can couple to the rubber, are very effective and suitable materials for this purpose. As the silica / silane reaction needs to be conducted during the mixing process, this technology brought a new challenge for mixing of the rubber compound, including now a chemical reaction process. Technical developments in order to realize an efficient and sufficient reaction have been carried with different focuses such as compounding, mixing conditions and also machine geometries as one of the current hot topics in the tire technology. More than 25 years have passed since the technology was firstly introduced, nowadays, silica filled compounds have been used very widely in the tire industry. However, in order to meet the increasing requirements for various tire performances, a further performance improvement is essential.

In addition, depending on the requirements of the desired tire performance, the magic triangle has to be enlarged in one direction in the actual tire development process. One of the important parameters to reach this, is the choice and blending of polymers. For instance, on one hand a high $\mathrm{T}_{\mathrm{g}}$-SBR is mainly used with a high blending ratio for emphasizing the wet performance, on the other hand the amount of $B R$ is increased when the abrasion resistance needs to be improved. Furthermore, the silica / silane system is mainly applied only for passenger car tire treads up to now. Inside the tread of trucks or busses a different polymer, $N R$, is used. But due to the fact that the silica-silane system does not react in the same way in a NR compound than in a solution SBR/BR compound, it is up to now not introduced inside the truck tread compound. Therefore, an understanding of the working mechanism between various types of polymers and silanes is crucial in order to overcome this limitation.

\section{1-2 Aim of this thesis}

The magic triangle can be enlarged further by improving a silica dispersion level especially when using a high surface area of silica, which is normally difficult to disperse in the rubber matrix because of the higher self-cohesive forces. Mercapto-silanes have received recently increased attention to improve the silica dispersion level in a rubber matrix. However, they cause also processability problems such as high Mooney viscosity and premature scorch. 
The mechanism behind them has not been clarified yet. Therefore, the aim of the present thesis is to find the way to improve the balance of the silica dispersion level, which leads to better viscoelastic properties, and the processability by understanding the reaction mechanism of silanes with polymers as well as the resulting internal reinforcing structure of the compounds. The reactivity and the mechanism between a silane and a polymer are expected to vary depending on the structure of them. Therefore, various combinations between different structural varied (model) polymers and silanes are investigated. These evaluations should lead to a solution to overcome the limitations in the use of mercaptosilanes in tire tread compounds. The clarification of the relationship between the reinforcing structure and compound properties including the processability would lead to the ideal internal structure to balance all properties on the highest possible level.

\section{1-3 Structure of this thesis}

The studies described in the present thesis focus on the reinforcing mechanism of silica filled rubber, as well as the related silane chemistry. Chapter 2 gives an overview of key points to provide a reinforcing effect such as silica characteristics itself, characteristics of silanes, the silica / silane reaction, the rubber / silane reaction and also the reaction mechanisms of sulfur and rubber.

This thesis encompasses five experimental chapters.

Chapter 3: the results of a model olefin study is described. Various types of olefins having different double bond structures in combination with mercapto-silane or sulfide silane are compared in terms of reactivity, reaction speed and the resulting structures. The model olefin which has only one double bond inside the structure is used in order to identify the reaction products and the mechanisms.

Chapter 4: the influences of silica, $\mathrm{ZnO}, \mathrm{CBS}$ and sulfur on the reaction between mercaptosilane and model olefin are investigated. By identifying all influencing parameters and ingredients on the reaction, the way to control it adequately is tried to find.

Chapter 5: the reactions between model substances which have more than one double bond in the structure and two silanes respectively are investigated. Two liquid butadienes 
which have a different vinyl content and squalene which has an isoprene structure (but with trans-double bonds) are used as model substances. As these systems are considered to be much closer to the actual rubber compound compared to the model olefin systems, by comparing the results here with the one in Chapter 3, the phenomena which might occur in the rubber compound systems are predicted.

Chapter 6: the mixing of the typical passenger car tire tread compounds which are SBR / BR blend silica filled compounds in combination with a mercapto-silane or a sulfide-silane is carried out, and the obtained internal structure and compound properties are explained based on the findings in the model study described in the previous chapters. The different silica reinforcing structures between two silane systems are summarized and the mechanism to provide a low tan $\delta$ and a high Mooney viscosity is explained.

Chapter 7: the mixing of silica filled NR or IR compounds instead of SBR / BR blends done in the previous chapter 6 in combination with a mercapto-silane or a sulfide- silane is carried out, and the results are compared with the one in the SBR / BR compounds. The characteristic differences between the NR or IR compound and the SBR / BR compounds are summarized. A possible mechanisms of the isoprene structure with silanes are proposed.

Chapter 8: an overall conclusion of this thesis is given. 


\section{Chapter 2}

\section{Literature survey; \\ Overview of reinforcing mechanism of silica filled rubber and silane chemistry}

\subsection{Introduction}

Viscoelasticity of a rubber compound for a tire tread considerably affects various tire performances. Since the time (frequency) - temperature superposition principle can be applied for the viscoelasticity of rubber compounds, several indications enabling to predict tire performances can be obtained by performing viscoelasticity measurements at different temperatures. The deformation frequency of a tire during the normal rotation is known to be ca. $10 \mathrm{~Hz}$ and for sliding over the unevenness surface of the road between $10^{3}-10^{6} \mathrm{~Hz}$, respectively. $10 \mathrm{~Hz}$ corresponds to ca. $60^{\circ} \mathrm{C}$ and $10^{3}-10^{6} \mathrm{~Hz}$ correspond to the range of -10 to $+10{ }^{\circ} \mathrm{C}$ under the normal condition of the viscoelasticity measurement (ex. $20 \mathrm{~Hz}$ ). Therefore, the values of $\tan \delta$ at these temperatures can be used as indicators for the rolling resistance and the wet grip performance of the tire. Herein, tan $\delta$ at $60^{\circ} \mathrm{C}$ works quite well to predict precisely the rolling resistance, while $\tan \delta$ at $0{ }^{\circ} \mathrm{C}$ has a certain limitation due to the complexity of the friction mechanism.[1] The relationship between viscoelastic properties of rubbers at different temperatures and tire performances are shown in Figure 2-1.[2]

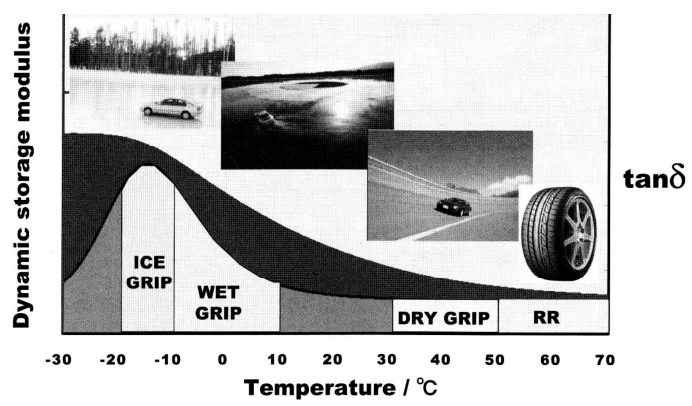

Figure 2-1: Relationship between viscoelastic properties and tire performances at different temperatures [2] 
In general, the following three reasons can be considered to cause an energy loss by the deformation of rubber compounds: [3]

1. Friction between filler clusters, which are used for the reinforcement, associated with the breakdown of the filler-filler network (Payne effect)

2. Mobility of free terminal groups in the rubber, which is more pronounced when the compound generates heat by the deformation: with increasing temperature, due to the kinetic energy which is released, this mobility of terminal group can be increased even more

\section{Friction between segments in the polymer chain}

In the case of rolling resistance, since $60^{\circ} \mathrm{C}$ is much higher than the $\mathrm{Tg}$ of the normally used compound, the first and second reason are dominant and especially the first reason plays a major role. Therefore, to reduce the filler-filler interaction with fixing the terminal group of the polymer in the rubber compound seems to be the most effective way to improve the rolling resistance. On the other hand, taking the wet grip into account, the third reason could be the most dominant factor for the tan $\delta$ value because of the closeness of the compound's $\mathrm{Tg}$ to $0^{\circ} \mathrm{C}$. In other words, the compound $\mathrm{Tg}$ significantly influences the wet grip performance.

It has been accepted that there are mainly two contributions for rubber friction on a rough surface, which are the hysteresis term and the adhesive term.[4] The hysteresis term is explained as an energy dissipation originating from the viscoelastic nature of rubber, as described above, during a periodical deformation caused by road surface asperities. The adhesion term corresponds to the shear force to overcome the adhesive force between molecular interactions by van der Waals bonding, which are generated at the actual rubber / road interface. It has been reported that silica filled compounds using silane coupling agents have a higher adhesive term compared to carbon black filled compounds.[5] Amino has suggested that this advantage attributes to the different internal structures of filler clusters inside the rubber, based on the observation by X-ray scattering, neutron scattering and Transmission Electron Microscopy (TEM). The proposed schematic models for the dispersed structure of carbon black and hydrophobized silica in rubber are shown in Figure 2-2. Both filler clusters form a fractal structure, however, that of hydrophobized silica does not develop agglomerates compared to that of carbon black and exists more independently 
in the rubber matrix. This internal structure of silica clusters enables polymer chains inside the compound to deform easily along the small surface asperities of the road, which leads to a higher actual contact area resulting in a higher adhesion friction. The isolated silica clusters can also generate lower friction between fillers resulting in low rolling resistance. In summary, dispersing silica clusters to the very fine level is the key point to improve the performance balance of the rolling resistance and the grip performance.

(a)

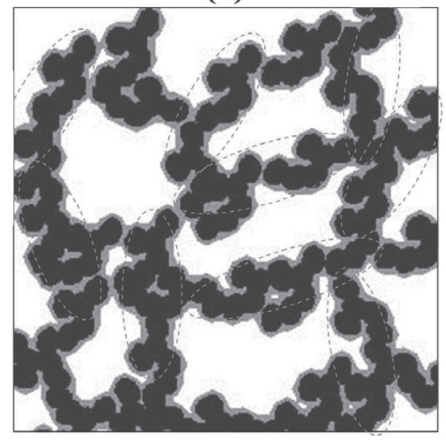

(b)

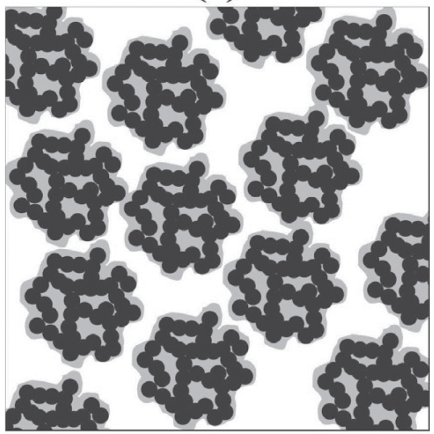

Figure 2-2: Proposed schematic models for dispersed structure of (a) carbon black and (b) silica in rubber [5]

The dispersion of silica into the rubber is conducted normally by using silane coupling agents which are accompanied by chemical reactions during mixing stages. For this, not only the properties of silica itself play an important role but also the mixing temperature, the structure of the silane and the presence or absence of other additives which may influence the reaction. Furthermore, the reinforcement of silica has been considered to be established finally through the vulcanization process involving the coupling reaction of rubber with silica via silane (which will be described in 2-3-5). However, these complicated mechanisms have not been fully understood. In the present chapter, a literature review regarding the reinforcing mechanism of the silica / silane system with the emphasis of possible chemical reactions will be displayed. 


\section{2-2 Silica as reinforcing filler}

\section{2-2-1 General Introduction of silica}

In the tire industry, hydrated amorphous silicas are normally used. They are prepared by a precipitation process of a water-soluble sodium silicate ("water-glass") with an acid. Various acids may be used, including mineral acids such as sulfuric acid and hydrochloric acid. By-product of the reaction is a sodium salt, which has to be washed out. The chemical reaction is carried out in precipitation tanks, equipped with stirrers to achieve excellent mixing of the components. Typical reaction times are between one and four hours at temperatures between 50 and $90^{\circ} \mathrm{C}$. However, the choice of temperature, $\mathrm{pH}$, dosing time and concentration of each raw material and mixing conditions determines the properties of the resulting silica regarding structure and surface area.[6-8]

In the very beginning of the precipitation process, dense and spherical colloidal isolated silica particles, named primary particles, are formed by condensation. With increasing time, the amount and size of these particles increase. Subsequently, when the viscosity comes to the gel-point where the salt concentration reaches a critical value, the primary particles start to form aggregates, characterized by Si-O-Si bonds between primary particles. These bonds are very stable. The ongoing process results in continuous growth of the number and size of these aggregates, until the aggregates are held together by reversible hydrogen bonds, the resulting particles are called agglomerates. The low salt concentration dominates a particle growth, while the high salt concentration leads to an aggregate growth as well as a preferred agglomeration process, as shown in Figure 2-3. The aggregate structure can be strengthened by increasing the number of siloxane bridges in a broader range between primary particles, which can occur in an aging process and / or an increase of the $\mathrm{pH}$ value. The strong aggregate structure can keep the structure by suppressing compression during the drying process resulting in larger void size inside aggregates. Moreover, the intense shearing during precipitation increases the void volume inside aggregates. [6-8] 


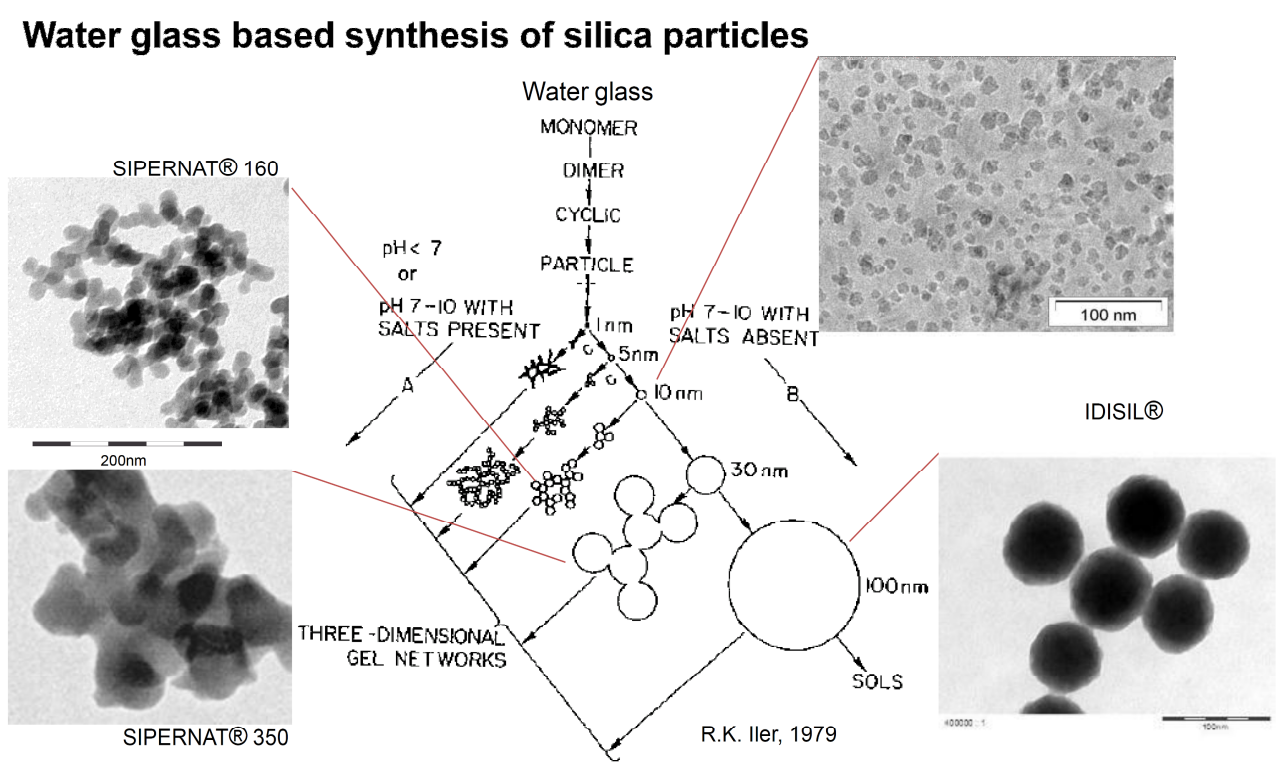

Figure 2-3: Production process of silica [9]

The typical classification of precipitated silica structure with the size is depicted in Figure 2-4.[8] However, since there is no strict boarder in terms of the size between aggregate and agglomerate, both of them are often called silica cluster.

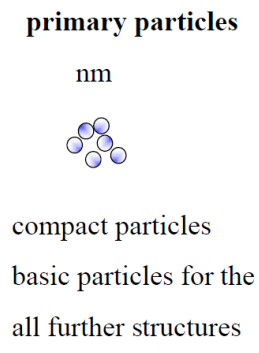

$$
\begin{aligned}
& \text { aggregates } \\
& \text { sub- } \mu \mathrm{m} \\
& 888008
\end{aligned}
$$

intra-linkage chemical bonding (covalent) agglomerates

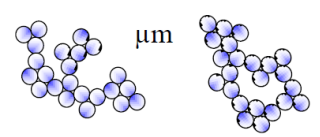

loosely connected;

hydrogen-bridges;

Figure 2-4: Classification of the silica structure [8]

\section{2-2-2 Characterization of silica}

The reinforcement ability and dispersibility of silica in rubber used for tire treads can be determined by combinations of many physical and chemical properties of the silica. In this 
chapter, the most significant properties of silica as well as the methods to characterize them are summarized.

\section{2-2-2-1 Silica surface area and structure}

The surface area of silica is one of the most crucial factors to determine the reinforcement ability of the silica, therefore has a big influence on the physical properties of filled rubber. The specific surface area of silica is generally determined by using two methods; the $\mathrm{N}_{2}$-adsorption, the Brunauer-Emmett-Teller method (BET), and the adsorption of $\mathrm{N}$-cetyl- $\mathrm{N}, \mathrm{N}, \mathrm{N}^{\prime}$-trimethylammonium-bromide, the CTAB method.[10] In the BET measurement, by using liquid nitrogen at low temperature the adsorbed amount of nitrogen gas is measured. This method measures the sum of the outer geometrical surface and the inner surface, that is, the surface within the porous silica structure, as shown in Figure 2-5. The micropores are generally small in size : $<2 \mathrm{~nm}$, into which only low molecular weight chemical compounds can penetrate, but polymers or coupling agents not. Therefore, this method has a potential to overestimate the accessible surface for coupling agents or polymers due to their inability to penetrate into these pores.

In case of the CTAB measurement, CTAB molecules are so large that they cannot penetrate into the micropores as shown in Figure 2.5. Therefore, the preferred adsorption site for these large CTAB molecules is the outer, geometrical surface, which correlates quite well with the surface area accessible to the rubber and silanes. As a result, the physical properties of the filled rubber compound strongly correlate with the CTAB surface area, better than with the BET surface area.[11]
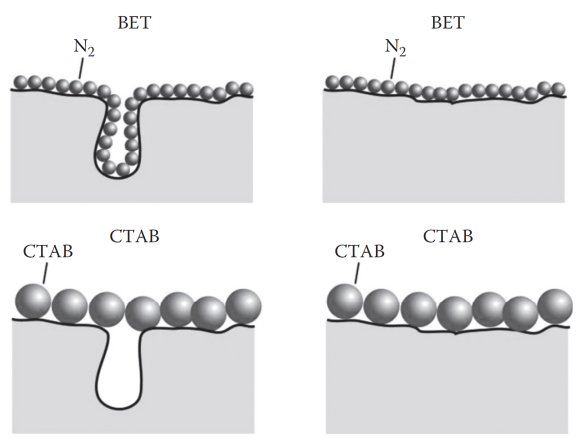

Figure 2-5: Difference of nitrogen and CTAB adsorption on porous and nonporous silica [10] 
During the production process of silica, as mentioned before, primary particles condense into aggregates which are the real reinforcing species in rubber compounds. When the particles are close together, the condensation between the primary particles can take place. The degree of condensation in aggregates, normally described as "structure", determines the free volume inside the aggregates, which is called "void volume". The most commonly used measurement of this "structure" is based on the adsorption of Dioctyl Adipate (DOA), generally called Oil Absorption Number (OAN). For a better dispersion, it is important for the silica aggregates to have sufficient amount of voids which can be easily penetrated by rubber polymers. Therefore, there is a general tendency that high dispersion types of silica has higher OAN values compared to conventional silica.[7]

Moreover, the void volume measurement has also been used to predict the silica dispersibility. During the measurement, the sample which is put in a cylindrical glass chamber is subject to the pressure to be coarse crushed. The void volume is calculated by the difference between the measured volume at a certain pressure and the theoretical volume of the sample. It was reported that the high dispersion types of silica has higher void volume together with less fragility to enable the polymer to penetrate into the voids compared to that of conventional types of silica.[7]

The more reliable prediction method for the silica dispersibility in rubber compounds is still a hot subject for the research. Recently, Grunert et al. introduced an analytical method to predict the silica dispersibility in combination with reproducing the silica dispersion process during mixing. In the method, silica is pre-treated in water by ultrasonic, subsequently the particle size distribution of silica during sedimentation is measured by $\mathrm{X}$ ray absorption. The amount of particle smaller than $2 \mu \mathrm{m}$ in the measurement correlates well with the achieved level of macro dispersion in the silica filled compound.[12]

\section{2-2-2-2 Silica surface chemistry}

The silica surface is composed of siloxane and silanol groups. The chemical characteristics of silica surface are mainly determined by the amount of silanol groups, the amount of absorbed water and the $\mathrm{pH}$. The silanol groups present on the surface of silica can be divided into three different types; depending on the hydroxyl group, which are;

- Isolated silanol group : a single hydroxyl group on a silicon atom

- Vicinal silanol group : two hydroxyl groups on adjacent silicon atoms 
- Geminal silanol group : two hydroxyl groups on the same silicon atom

Adjacent silanols such as the geminal type of silanol groups are highly capable of absorbing water. For highly dispersible silica, it is found that the geminal silanol content is less than ca. $20 \%$. In addition, a siloxane bridge is formed when one oxygen atom is shared by two silicon atoms as shown in Figure 2.6(4). The silanol groups are detectable by Infrared (IR) spectroscopy and / or ${ }^{29} \mathrm{Si}$ Nuclear Magnetic Resonance (NMR) spectroscopy as presented in Table 2.1.[13-16]

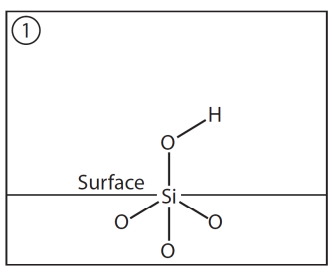

Isolated

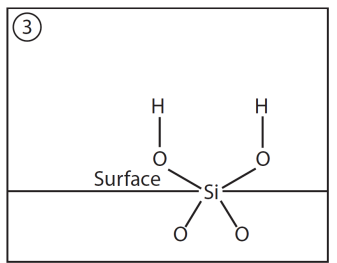

Geminal
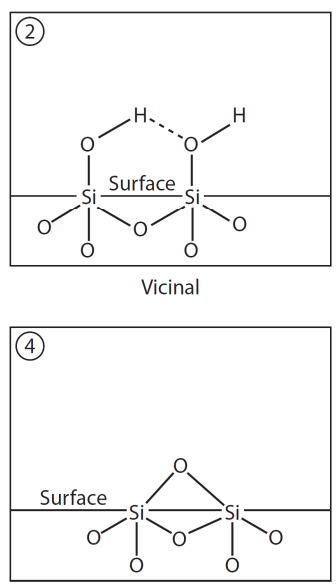

Siloxane bridge

Figure 2-6: Types of silanol groups on the silica surface

Table 2.1: Characterization of silanol groups by IR and ${ }^{29} \mathrm{Si} \mathrm{NMR}$

\begin{tabular}{lcc}
\hline \hline & Infrared band $\left(\mathrm{cm}^{-1}\right)$ & ${ }^{29}$ Si NMR $(\mathrm{ppm})$ \\
\hline \hline isolated silanol & 3740 & 100 \\
vicinal silanol & 3500 & 110 \\
geminal silanol & $3700-3740$ & 90 \\
siloxane bridge & - & 110 \\
inner silanol & $3670-3680$ & - \\
adsorbed water & 1630,5260 & - \\
\hline \hline
\end{tabular}

Blume reported that isolated and geminal silanol groups are responsible for the reaction with alkoxy groups of silanes (which will be described in 2-3-2).[14] The high dispersible silica ULTRASIL ${ }^{\circledR} 7000 \mathrm{GR}$ has a lower absolute content of silanol groups per $\mathrm{nm}^{2}$, but a 
higher content of isolated silanol groups in comparison with the conventional silica ULTRASIL ${ }^{\circledR}$ VN3.[13] Molecular modeling studies have shown that if the distance between two adjacent silanol groups is below $2.5 \AA$, they form a vicinal silanol group with stabilization by a hydrogen bond. In other words, isolated silanol groups can be formed with having over $2.5 \AA$ distance from the adjacent silanol group. However, for the ethoxy group, which is the most widely used alkoxy group in silane coupling agents for the tire industry, the requisite minimum distance to react with both adjacent silanol groups is $4 \AA$, which means that the reactivity of the silica with silane coupling agents depends not only on the isolated silanol number but also on the distance that separates these silanol sites.[13]

\section{2-3 Silane Chemistry}

\section{2-3-1 Introduction of silane}

Silica is incompatible with the non-polar tire polymers such as polybutadiene (BR) and styrene / butadiene copolymers (SBR) due to the high concentration of polar hydroxyl groups at the silica surface. Therefore, the application of an organofunctional silane is necessary to overcome the polarity differences in order to achieve a higher desirable silica dispersion level in the rubber compound.

Mainly, bi-functional-organosilanes based on the structure shown in Figure 2-4 are used.

$$
\mathrm{R}=\mathrm{CH}_{3}, \mathrm{C}_{2} \mathrm{H}_{5} ; \mathrm{X}=\text { functional group }
$$

Figure 2-4: Basic structure of bi-functional-organosilanes [10]

One function is for the hydrophobation of the silica surface, which is achieved by the reaction of hydrolyzable groups such as alkoxy groups of the silane with the silanol groups on the silica surface. Normally, triethoxysilanes are selected because the released ethanol is classified as non-toxic. The hydrophobized silica becomes easier to be mixed with nonpolar tire polymers. Another function of the silane is to couple to the rubber. The organofunctional part of the silane such as sulfide, mercapto, amino, epoxy, vinyl or isocyanate groups can provide the chemical bonding with the polymer. By these two coupling reactions, a chemical bridge between the silica and the rubber is formed, therefore, the reinforcement effect of silica can be increased significantly. 
The bi-functional-organosilanes which have been most widely used in the tire industry so far are bis(triethoxysilylpropyl)tetrasulfide (TESPT) and bis(triethoxysilylpropyl)disulfide (TESPD). The structures of them are shown in Figure 2-5. They are actually not pure tetraor di-sulfides but a mixture of polysulfides. The average sulfur rank of TESPT is ca. 3.8 and that of TESPD is between 2.15 and 2.35.

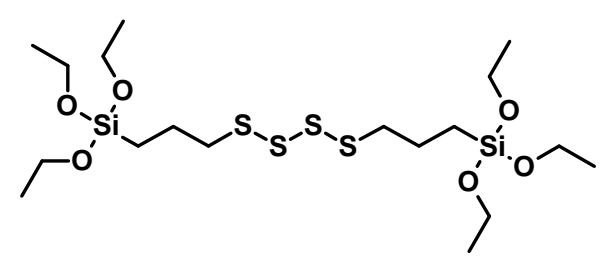

(a)

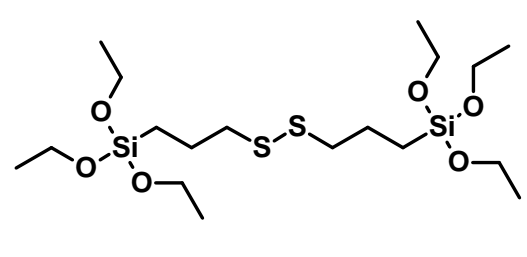

(b)

Figure 2-5: The structure of (a) Bis(triethoxysilylpropyl)tetrasulfide (TESPT),

(b) bis(triethoxysilylpropyl)disulfide (TESPD) [10]

\section{2-3-2 Mechanism of the silanization reaction}

After the adsorption of the silane coupling agent onto the silica surface, hydroxyl groups on the silica surface start reacting with alkoxyl groups of the silane coupling agent. This reaction is named "silanization" and was investigated intensively.[17-20]

It was postulated that the reaction mechanism of the silanization can be divided into two stages, which are a primary and secondary reaction as schematically summarized in Figure 2-6.[21,22] The primary step is the reaction of one alkoxyl group of the silane with preferably an isolated or a geminal silanol group at the silica surface.[13] Two possible mechanisms were reported as shown in Figure 2-6(a).

1. Direct reaction of silanol groups of the silica with the alkoxy group of the coupling agent

2. Hydrolysis of the alkoxy group to form a reactive hydroxyl-group with the release of ethanol.

The reactions (in No. 2) occur slowly on the silica surface in the presence of water. It has been reported that the amount of ethanol generated during the mixing process increases with an increasing moisture content of the silica, but that a moisture content higher than 
$7 \%$ does not lead to any further acceleration. Furthermore, the reaction rate of hydrolysis increases with increasing temperature, as well as by using a catalytic agent such as an acidic or alkaline medium. After the hydrolysis, the activated silane is capable of reacting with silanol groups on the silica surface following a condensation reaction. The reaction rate of the condensation is faster than that of the hydrolysis. This means that the hydrolysis reaction is the rate-determining step of the primary reaction of the silanization.

After the primary reaction an intermolecular condensation between silanes on the silica surface, named as secondary reaction, takes place as shown in Figure 2-6(b), caused by unreacted, adjacent ethoxy groups of the silanes. The reaction rate of the secondary reaction is rather slow compared to that of the primary reaction. The secondary reaction is also accelerated by water and increasing temperature.

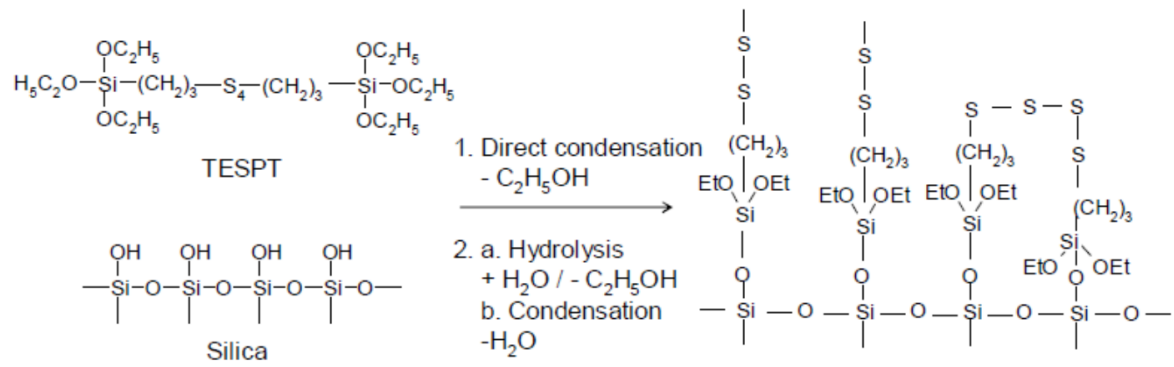

(a) : Primary reaction of silica with TESPT

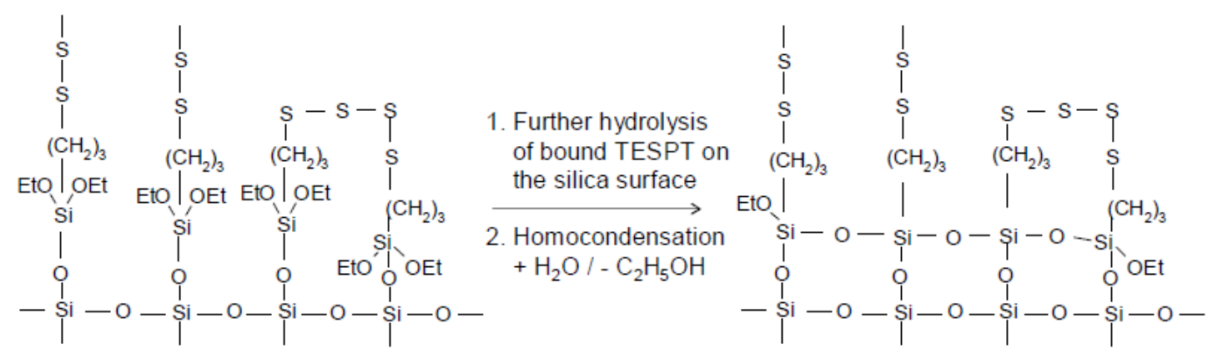

(b) : Secondary reaction of silica with TESPT

Figure 2-6: Reaction mechanism of the silanization [21,22] 


\section{2-3-3 Kinetics of the silanization reaction}

Kinetic studies were carried out to increase the understanding of the silanization mechanism. The kinetic parameters of the silanization reaction can be estimated by a quantitative analysis of the released amount of ethanol during processing or by the remaining amount of silane after processing. Based on the assumption that the primary reaction is a first order reaction, the kinetic parameters of the reaction between TESPT and silica were estimated as follows;

$$
\begin{gathered}
-\frac{d[\text { TESPT }]}{d t}=k_{a}[\text { TESPT }]=\frac{1}{2} \frac{d[E t O H]}{d t} \\
\ln k_{a}=\ln A-\frac{E_{a}}{R T}
\end{gathered}
$$

where $t$ is the time, $k_{a}$ is the rate constant of the primary reaction, $E_{a}$ is the activation energy, $R$ is the gas constant and $T$ is the absolute temperature. In this equation, it is assumed that the conversion of TESPT accompanies the reaction of one ethoxy group on each triethoxysilyl group at both sides in a molecule at the same time, therefore, 1/2 is considered in equation (1) for the change of the EtOH-concentration over the time. For the reaction of TESPT with silica, the kinetic parameters are summarized in Table 2.3. The rate of the primary reaction increases with increasing reaction temperature. The activation energy by using the Arrhenius equation (2) was calculated as $47 \mathrm{~kJ} / \mathrm{mol}$.[17]

Table 2.3 Kinetic rate constants for the primary reaction $k_{1}$ and the secondary reaction $k_{2}$ at different temperatures [19]

\begin{tabular}{llll}
\hline Temp. $\left({ }^{\circ} \mathrm{C}\right)$ & 120 & 140 & 160 \\
\hline $\mathrm{k}_{1}\left(\mathrm{~min}^{-1}\right)$ & 0.061 & 0.122 & 0.229 \\
$\mathrm{k}_{2}\left(\mathrm{~min}^{-1}\right)$ & 0.006 & 0.008 & 0.012 \\
\hline
\end{tabular}

The reaction rate between silica and silane is one of the crucial factors to increase the achievable silica dispersion level in the compound. However, since it is necessary for silanes to adsorb on the silica surface first in order to start the silanization, the adsorbability of silanes is the most dominant factor to determine the total silanization reaction rate.[13] 
Blume investigated the reaction rate between various types of silane and silica by observing the turnover of silanes during a reaction in a model system, and reported as follows; a shorter alkyl chain of the silane leads to a higher silanization rate. Furthermore, linear alkyl chain silanes react faster than branched ones. This suggests the significant role of the steric hindrance effect of the alkyl chain of the silanes in the reaction mechanism. The longer or the branched alkyl chain decrease the possibility for alkoxy groups to be adsorbed onto the silanol group on the silica surface. In addition, the presence of a thiocyanato-group, the vinyl-group and long and polymeric amphiphilic substituents enhances the silica / silane reaction rate significantly. These results indicate that the chemical structure of the whole silane dominates the silica / silane reaction rate (Figure27).[23]

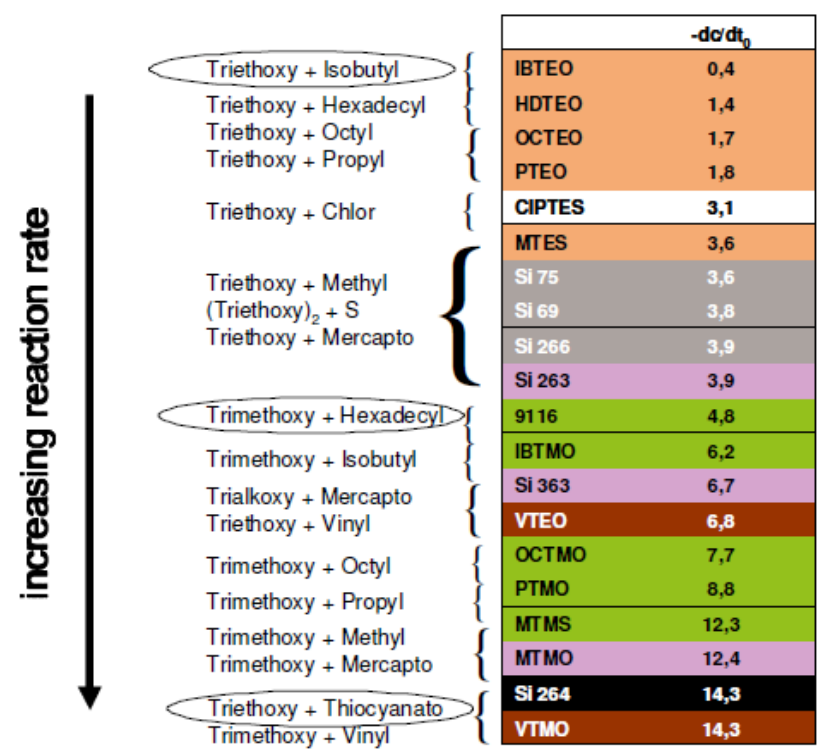

Figure 2-7: Start reaction rates of various types of silane with silica [23]

The influence of zinc oxide ( $\mathrm{ZnO}$ ) and stearic acid (St-Ac), which act as curing activators, on the rate of the silica / silane reaction has also been reported.[24-27] These chemicals can be adsorbed onto the silica surface, therefore compete with the adsorption of silanes. As a result, the rate of silica / silane reaction decreases resulting in a reduced hydrophobation effect. Furthermore, the effectiveness of the above mentioned chemicals and additionally of the accelerators, which can be also adsorbed on the silica surface during the vulcanization process, decreases, which causes a reduction of the cross-link density as 
well as the vulcanization rate. As another reason for reducing the silca / silane reaction rate in the system with $\mathrm{ZnO}$, the decrease of the effective amount of silane by formation of a complex between the sulfur part of the silane and $\mathrm{ZnO}$, has been proposed. [25]

\section{2-3-4 Silanization in natural rubber system}

Natural rubber (NR) has a lot of unique properties which are completely different from synthetic rubbers. One of them is the higher content of non-rubber constituents. It was found that those non-rubber constituents influence the silanization rate.[28,33]

The fundamental structure of a linear NR chain consists of a long sequence of 1000 to 3000 cis-1,4 isoprene units, with specific other groups at the $\alpha$ - and $\omega$-terminals (Figure 2 8). The $\alpha$-terminal consists of the reactive groups such as hydroxyl, carbonyl and aldehyde groups, which are associated with the amino-acid in protein, and the phosphates at the $\omega$ terminal couple with phospholipids via hydrogen and / or ionic bond (Figure 2-9). Both of them provide an effect like a cross-linking points, which results in higher molecular weight as well as higher gel content in NR.[29-32]

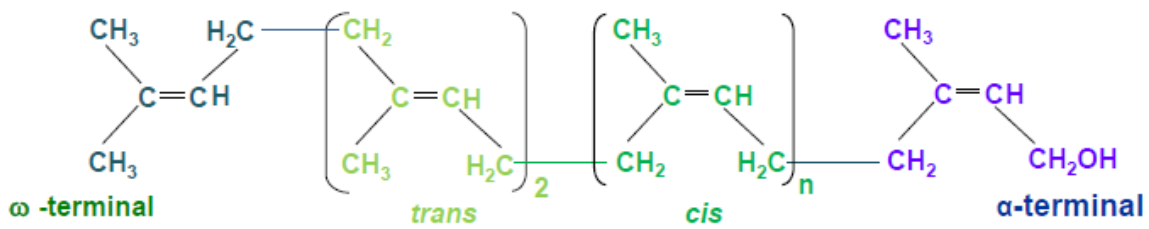

2 trans-1,4 isoprene units vs $1000-3000$ cis-1,4 isoprene units

Figure 2-8 : Basic structure of natural rubber [30,32]

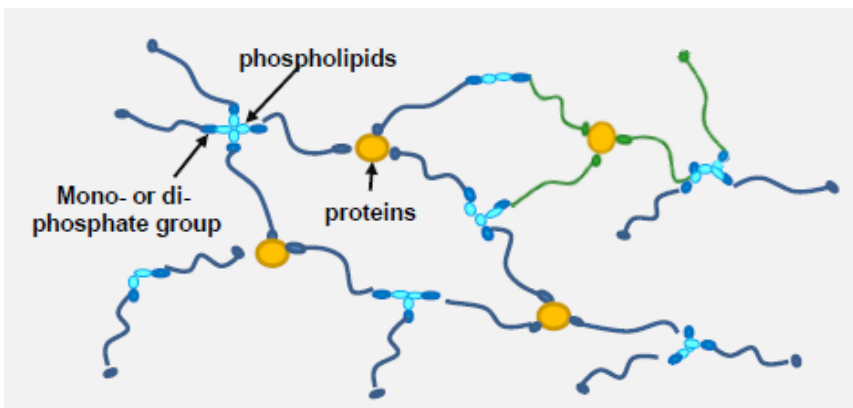

Figure 2-9: Linear rubber chain structure with the naturally occurring network associated with proteins and phospholipids [29,31] 
The protein content of NR varies upon its source and methods of the production. The nitrogen content of NR is related to the protein level. Typical raw NR has a nitrogen content in the range of $0.3-0.6 \%$. One of the most successful attempts to purify NR is the "Deproteinized Natural Rubber" (DPNR), which is produced by the treatment of natural rubber latex with bioenzyme (proteinase) which transferred the proteins into water soluble forms to separate them from the rubber phase. In comparison to this, skim rubber which is produced by coagulation of the serum phase after the centrifugation with sulfuric acid has a very high protein content, where the nitrogen content has values in the range of $1.5-2.5 \%$.

Sarkawi investigated the influence of the protein content on silica filled NR systems both with and without a silane coupling agent by using NR, DPNR and Skim Rubber. In the absence of silane, she found that the proteins are adsorbed at the silica surface. But as soon as silanes are present, there is a competition between the adsorption of the silanes and the proteins on the silianol groups of the silica, therefore, the silanization is getting suppressed with increasing amount of proteins.[28]

Blume also investigated the influence of an amino acid on the silanization reaction using a model system, and reached the same conclusions that higher amounts of an amino acid causes a decrease of the reaction efficiency of the silanization reaction because of a competition between the amino acid and the silane for the adsorption on the silica surface.[33]

\section{2-3-5 Silane / Rubber coupling}

In order to implement the reinforcing effect of silica, the sulfur part of the silane, which is already attached to the silica, has to react with the rubber. Many studies were carried out to investigate the reaction mechanism-of the sulfur part of TESPT or TESPD with rubber. The investigation of the reaction in real rubber compounds is rather complicated due to many possible structures as reaction products. Therefore, model study techniques have been applied widely. Using small molecular weight chemicals such as olefins and additionally squalene which is a model substance of rubber (Figure 2-10), instead of rubber enables the detection of the educts as well as of the reaction products by different analytical methods. These studies have helped to understand the principle reaction mechanism better and supported the better understanding of the processes in rubber as well. 


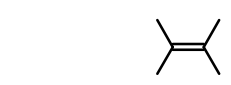

2,3-dimethyl-2-butene (tetra-methyl-ethylene (TME))

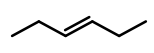

trans-3-hexane (T3H)

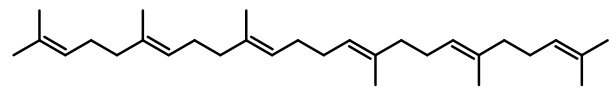

Squalene

Figure 2-10 Examples of small molecular weight chemicals for model study

In the case of TESPT and TESPD, which are widely used in the tire industry, it is possible to detect the concentration of each component having different sulfur chain length by Highperformance Liquid Chromatography (HPLC). Therefore, the method to track the change of the sulfur length distribution and the total remaining amount of silane (to determine the reaction efficiency) by HPLC under the heating condition has been often used to understand the reaction mechanism of the silanes in the model study.

For example, by heating only sulfur and TESPT, the ratio of longer sulfur silanes increases by heating, which leads to an increase of the average sulfur length of TESPT, as shown in Figure 2-11. This behavior was confirmed by other researchers.[34] Therefore, it is assumed that TESPT acts as a sulfur acceptor showing a fast incorporation rate.[35]

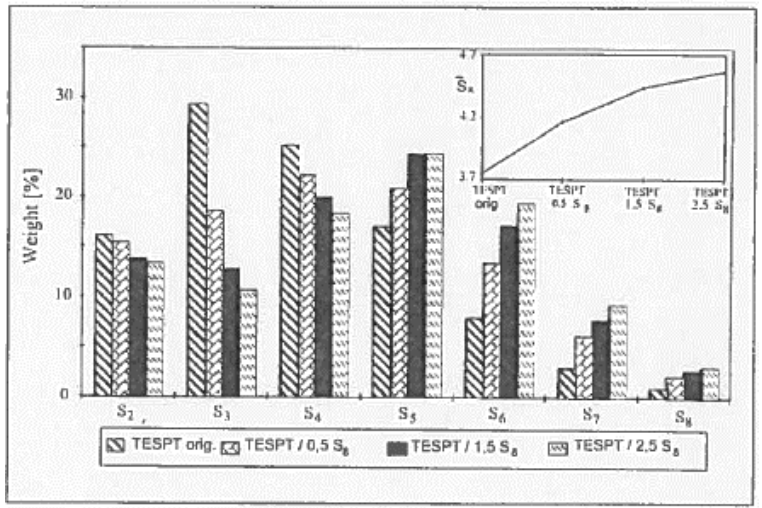

Figure 2-11: Influence of sulfur on the TESPT polysulfide chain distribution and the average sulfur chain length (small figure) by heating at $150{ }^{\circ} \mathrm{C}$ for $40 \mathrm{~min}$. [35]

However, when the TESPT is heated together with the accelerator N-Cyclohexyl-2benzothiazole sulfenamide (CBS), stearic acid (St-Ac) and $\mathrm{ZnO}$ but without sulfur, a different behavior is observed. The average sulfur length of silanes is under these conditions shortened by heating, and the total concentration of TESPT is also decreased (Figure 212).[35] This different behavior is explained in the literature as following: CBS reacts 
preferably with the long chain polysulfide silanes forming accelerator / TESPT reaction products.

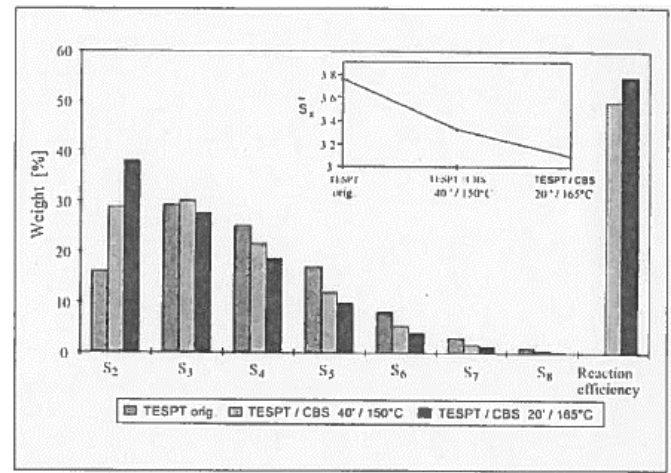

Figure 2-12: Change of the TESPT polysulfide chain distribution and the average sulfur chain length after heating at $150{ }^{\circ} \mathrm{C}$ for $40 \mathrm{~min}$ or $165^{\circ} \mathrm{C}$ for $20 \mathrm{~min}$ in the presence of CBS, stearic acid and zinc oxide (without sulfur) [35]

This intermediate product between TESPT and CBS is assumed to react directly with the rubber without adding free sulfur. This is supported by the following results. The reaction amount between TESPT and CBS in the absence of an olefin was $50 \%$ after heating at 165 ${ }^{\circ} \mathrm{C}$ for 20 minutes, but by adding squalene (compare Figure 2-10), which contains several double bonds, the reaction efficiency of TESPT increases up to $80 \%$ having the same heating conditions (compare Figure 2-13 and 14).

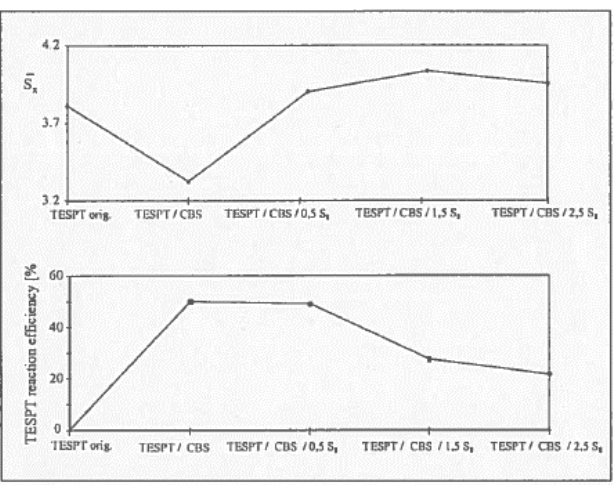

Figure 2-13: Average sulfur chain length and reaction efficiency of TESPT after heating at $165{ }^{\circ} \mathrm{C}$ for 20 min with CBS, $\mathrm{ZnO}$ and St-Ac in the absence and presence of sulfur [35]

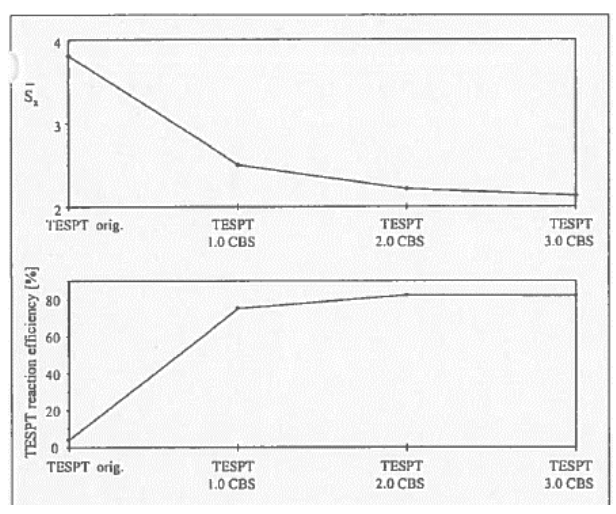

Figure 2-14: Average sulfur chain length and reaction efficiency of TESPT after heating at $165{ }^{\circ} \mathrm{C}$ for $20 \mathrm{~min}$ with squalene, CBS, $\mathrm{ZnO}$ and St-Ac in the absence of sulfur [35] 
Furthermore, when a mixture of sulfur (with varying contents), CBS, ZnO, St-Ac and TESPT are heated at $165^{\circ} \mathrm{C}$ for $20 \mathrm{~min}$ (again in the absence of squalene) the average sulfur length is increased and the total amount of TESPT is decreased at the same time as shown in Figure 2-13. Therefore, it was concluded that sulfur has reacted with both, TESPT and CBS, and that those reactions are competing.

In the case of TESPD, the incorporation rate of sulfur into the di-sulfide structure is very slow due to the higher dissociation energy of the di-sulfide bond compared to the polysulfide bond and elementary sulfur in the form of a $S_{8}$ ring. Therefore, almost no incorporation of sulfur occurs when heating only sulfur and di-sulfide silane even at a higher temperature than $160{ }^{\circ} \mathrm{C}$. The presence of sulfur and additionally CBS, ZnO and St-Ac together with higher temperature than $140{ }^{\circ} \mathrm{C}$ is necessary to enable the di-sulfide silane to incorporate sulfur into the structure. Moreover, to investigate the possible direct coupling from TESPD to an olefin, TESPD was heated only with CBS, ZnO and St-Ac and additionally with squalene, but in the absence of sulfur. No coupling reaction could be observed. Only in the presence of sulfur, after the incorporation of sulfur inside the $\mathrm{S}_{2}$ bond, the subsequent reaction with squalene becomes possible.[36]

All the results of the model system can be used now to understand better the reaction mechanisms inside the rubber compound. The di-sulfide accelerator reacts with the tetrasulfide group of the TESPT / silica intermediate product and forms an asymmetric polysulfide. In the subsequent reaction the polysulfide is added to the rubber in allylic position by releasing mercapto benzthiazole (MBT) (Figure 2-15).

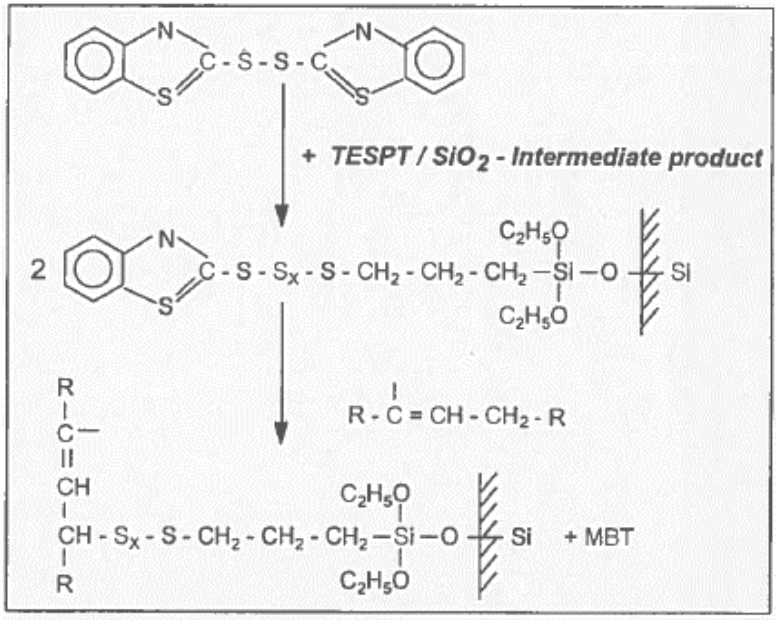

Figure 2-15:

Possible mechanism of silane crosslinking with CBS without sulfur [35] 
The above explained reaction mechanisms of silanes to a rubber were the cases which contain crosslinking agents. However, it has also been considered that the TESPT sulfur function is able to react thermally with rubber even in the absence of crosslinking agents. For example, the Mooney viscosity at the $1^{\text {st }}$ mixing stage increases when the mixing temperatures are above $150{ }^{\circ} \mathrm{C}$ in the SBR / BR silica filled rubber compounds. This increase goes hand in hand with an increase in the bound rubber content (Figure 2-16, 17). Since the $\mathrm{G}^{\prime}$ at high strain increases at the same temperature, it is assumed that the dissociated sulfur containing part of the silane and / or active sulfur released from silane starts to react with rubber at that temperature, which leads to the formation of silica-silane-rubber covalent bonds and / or rubber crosslinked products. This is called "pre-scorch" and might cause the processability difficulties.[22,37]
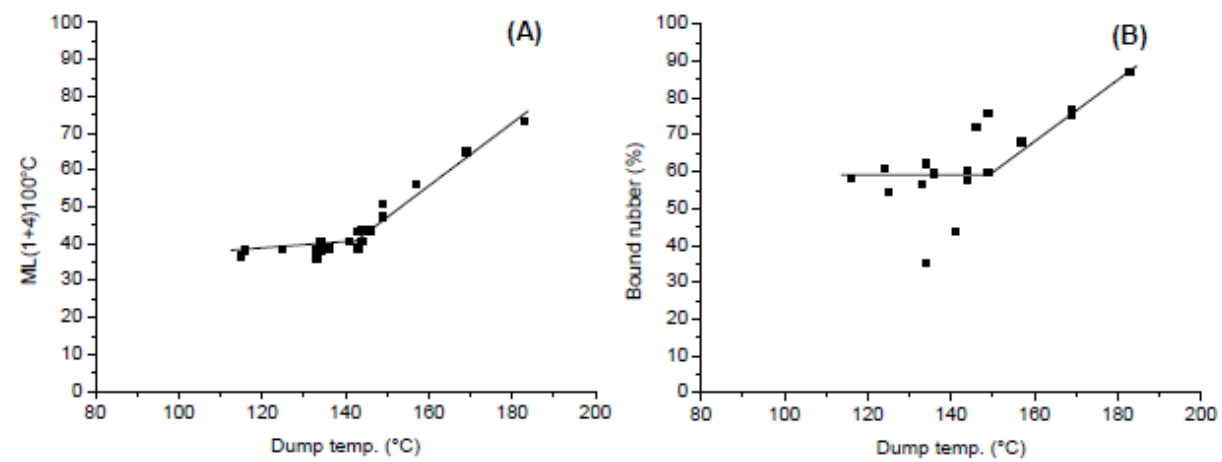

Figure 2-16: Mooney viscosity (A) and bound rubber (B) as a function of dump temperature [37]
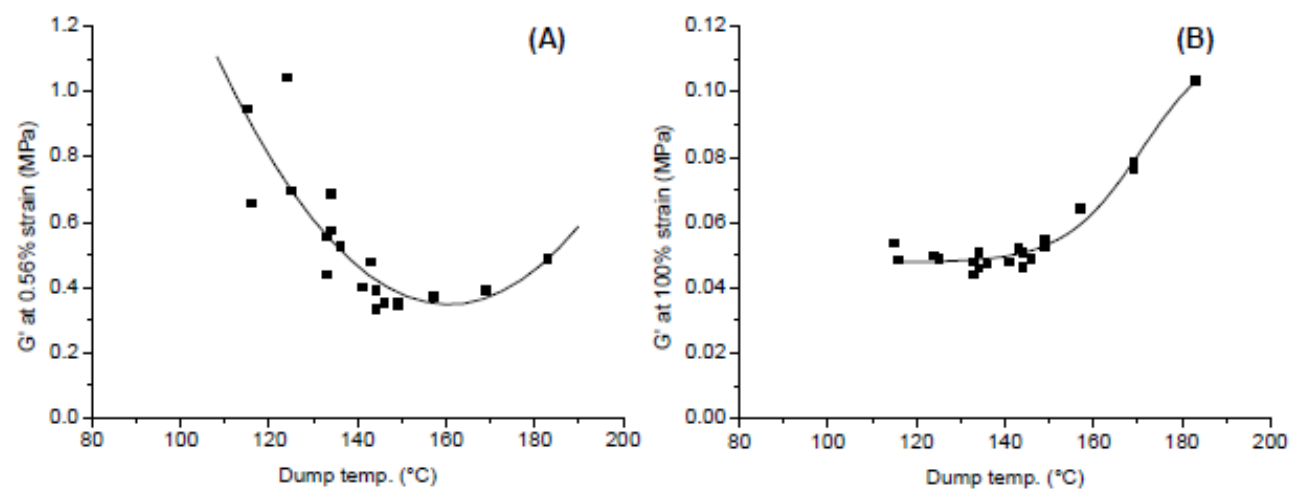

Figure 2-17: Influence of dump temperature on the storage modulus at $0.56 \%$ strain $(A)$ and $100 \%$ strain (B) [37] 
Starting temperature of those reactions could be much lower in NR / silica filled compounds. The cure curve measurement of a NR, TESPT and DPG mixture shows a torque rise even at $120^{\circ} \mathrm{C}$, while it starts from $150{ }^{\circ} \mathrm{C}$ in the case of the SBR / TESPT mixture, as shown in Figure 2-18.[38]
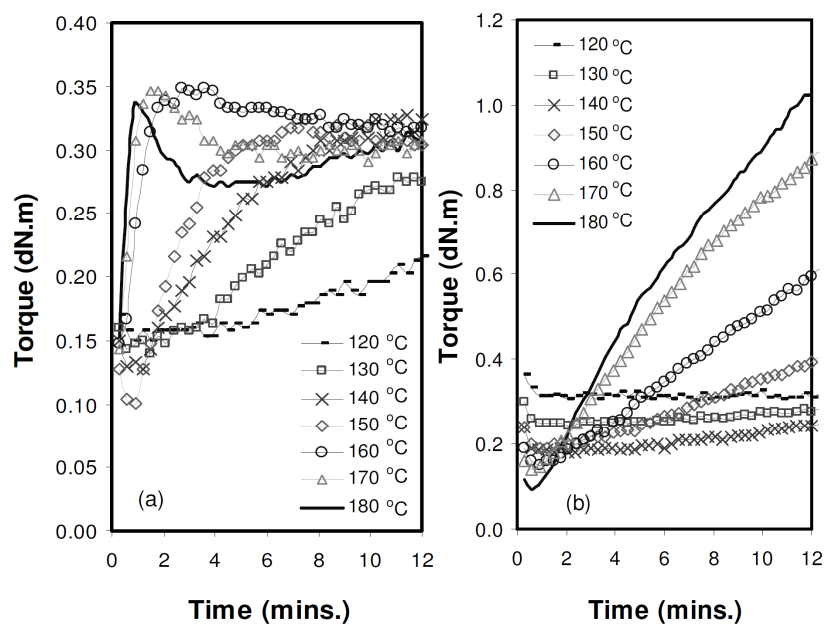

Figure 2-18: Cure characteristics of (a): NR and (b): SBR compounds, in the presence of TESPT (5.0 phr) and DPG (1.5 phr) at different cure temperatures [38]

Model compound studies were also carried out to investigate this "pre-scorch" reaction further.[34,35,39] By mixing only TESPT and squalene (which is a model substance of rubber), the average sulfur length of the silane decreases. This effect is the higher the higher the temperature is (Figure 2-19 and 2-20). The reaction efficiency of TESPT also increases with increasing heating temperature, especially above $150^{\circ} \mathrm{C}$. Silanes with a longer sulfur chain length, especially above $S_{5}$, show a higher reactivity and after 1 hour of heating at 170 ${ }^{\circ} \mathrm{C}$, almost no silane with a sulfur chain length higher than four can be detected. The reaction efficiency of TESPT at $170{ }^{\circ} \mathrm{C}$ reaches about 30\%.[35] 


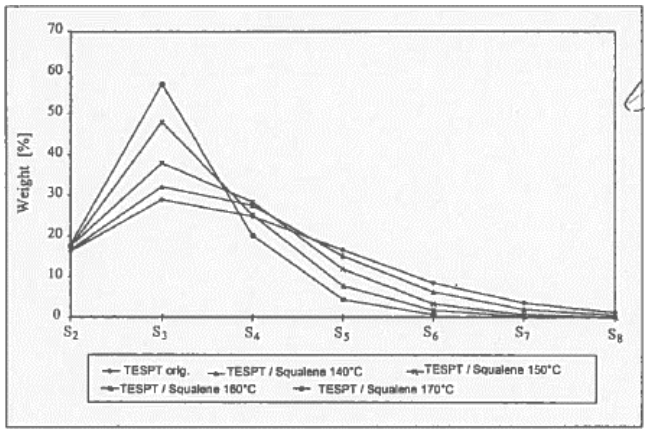

Figure 2-19: Influence of the reaction temperature on the TESPT polysulfide distribution in the presence of squalene [35]

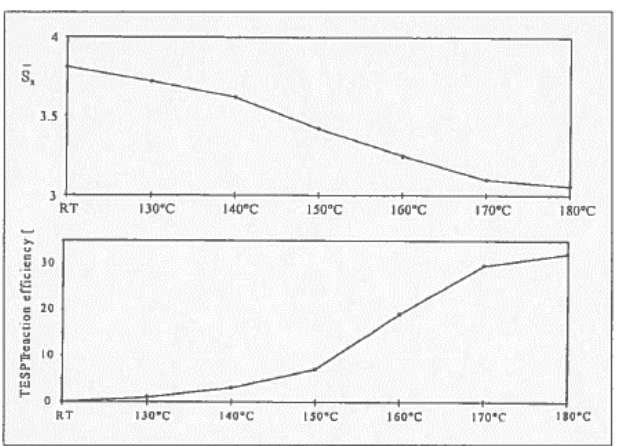

Figure 2-20: Average sulfur chain length and TESPT reaction efficiency depending on the temperature in the presence of squalene [35]

Debnath carried out a study using TME (tetra-methyl-ethylene) as a model compound for rubber in order to understand the silane-rubber reaction by comparing the reaction of TME and TESPT with the reaction between TME and sulfur. By heating TME with sulfur at $140{ }^{\circ} \mathrm{C}$, reaction products resulting from the combination of two TME molecules where sulfur was added in the allylic position with different sulfur length were detected by HPLC (Figure 221). Peaks from this investigation were also detected when TME was heated with TESPT (Figure 2-22). Therefore, it can be assumed that TESPT releases active sulfur resulting in the formation of a sulfur bridge between two TME molecules.[34]

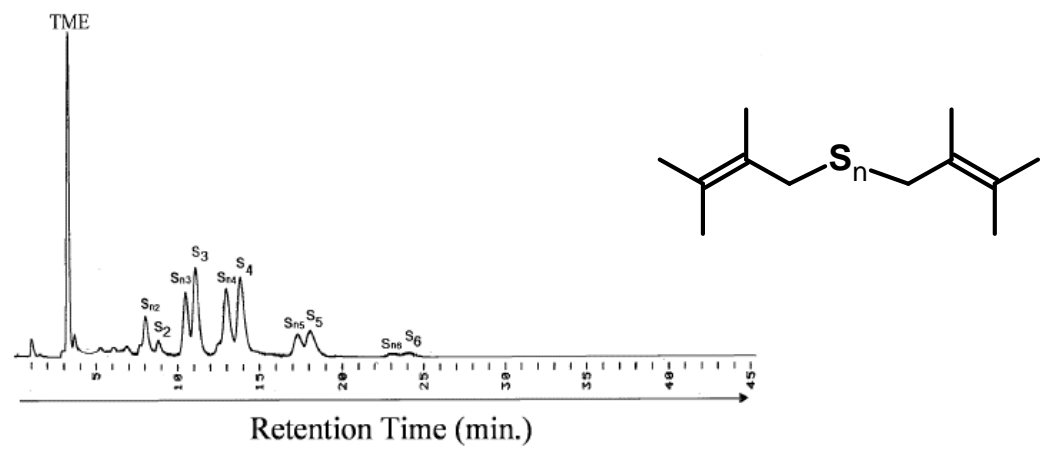

Figure 2-21: HPLC chromatogram of the reaction product of TME and sulfur heated at 140 ${ }^{\circ} \mathrm{C}$ for 1 hour and proposed structure of reaction product [34] 


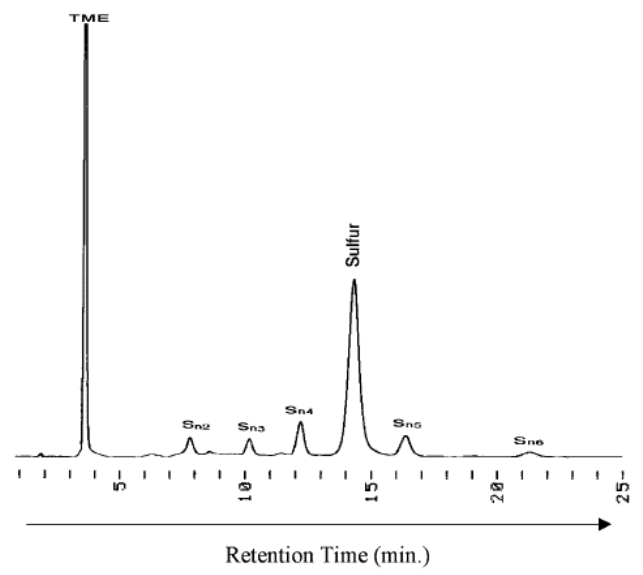

Figure 2-22: HPLC chromatogram of TESPT after heating with TME at $140{ }^{\circ} \mathrm{C}$ for 1 hour 「34]

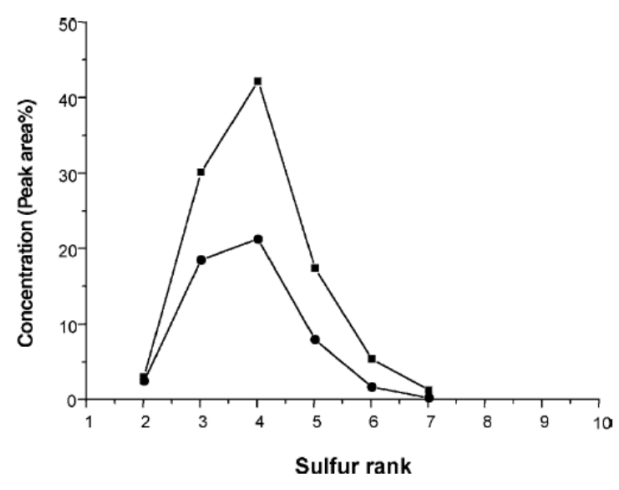

Figure 2-23: Comparison of sulfur rank in TESPT with (O) and without $(\boldsymbol{\square})$ TME at $140^{\circ} \mathrm{C}$ after 1 hour [34]

Hayen also confirmed the generation of the cross-linking products shown in Figure 2-21 with sulfur length of $\mathrm{x}=1$ and 2 in the TME / TESPT sample after heating at $140{ }^{\circ} \mathrm{C}$ for 30 minutes using LC/MS with the coordination ion spray-mass technique. Furthermore, he investigated the reaction mechanism of the olefin trans-3-hexene (T3H) (its structure is shown in Figure 2-10) with TESPT. He proposed, based on the mass data, the generation of adducts formed by the thermal cleavage of TESPT and subsequent addition of the formed $(\mathrm{EtO})_{3} \mathrm{Si}\left(\mathrm{CH}_{2}\right)_{3} \mathrm{~S}_{\mathrm{x}}(\mathrm{x}=2-5)$ to a T3H molecule, as shown in Figure 2-24. But unlike the TME system, the cross-linking products of two T3H via sulfur bridges comparable to the structure shown in Figure 2-21 were not detected. An explanation for this different behavior is not given.[39]

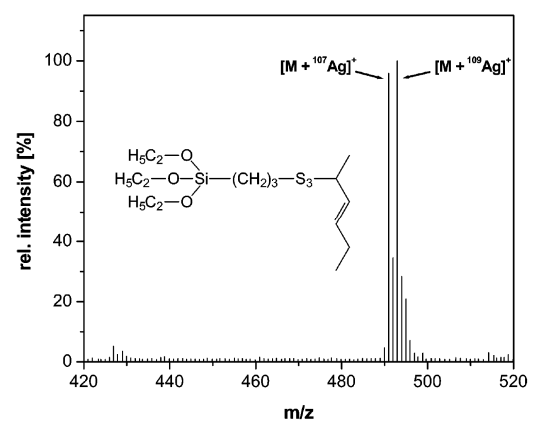

Figure 2-24: Mass spectrum and the proposed structure of one of the reaction products in the T3H / TESPT sample after heating at $140{ }^{\circ} \mathrm{C}$ for $30 \mathrm{~min}$ [39] 
It is known from the literature that the structure of the olefin can influence the reaction speed with sulfur due to the influence of the alkene stability.[40] This stability of alkenes can be depicted in the following way, starting with the most stable alkenes and ending with the less stable, the latter being the most reactive structure:

$$
\mathrm{R}_{2} \mathrm{C}=\mathrm{CR}_{2}>\mathrm{R}_{2} \mathrm{C}=\mathrm{CHR}>\mathrm{R}_{2} \mathrm{C}=\mathrm{CH}_{2} \sim \text { trans- } \mathrm{RCH}=\mathrm{CHR}>\text { cis- } \mathrm{RCH}=\mathrm{CHR}>\mathrm{RCH}=\mathrm{CH}_{2}>\mathrm{H}_{2} \mathrm{C}=\mathrm{CH}_{2}
$$

where $\mathrm{R}$ is alkyl. An increase in the number of alkyl substituents results in more stable alkenes, which is due to the inductive effect of alkyl groups. The difference in stability between the trans- and cis-configuration is mainly due to a steric hindrance of the alkyl substituents. The above mentioned order of the reactivity of alkenes with sulfur was confirmed by comparing three types of alkenes, 3-methyl-1-pentene (3m1p), trans-3hexene (T3H) and TME.[37] The highest remaining amount of sulfur after the reaction was detected for TME, the lowest for $3 \mathrm{~m} 1 \mathrm{p}$. It is assumed that the reaction rate of active sulfur released from TESPT with different alkenes shows the same dependence on the alkene structure than the reaction rate between different alkenes and pure sulfur.

As a consequence of several investigations known from the literature,[34,35,37,39] two possibilities have been suggested as reaction products between olefins and TESPT, which are the cross-linking products of two olefins combined via sulfur atom(s) released from TESPT, and the adducts of dissociated TESPT by heating to an olefin. It was implied that the preferred reaction depends on the structure of the olefin, however, it is not clear which reaction is preferred for which olefin. Moreover, since the quantitative data have not been obtained so far, it cannot be finally concluded how many direct bonds between the silica and the rubber via silane can be formed during the mixing process.

\section{2-3-6 Mercapto-silanes}

Recently, with an increasing demand to extend the magic triangle of tire performances, mercapto-silanes have received an increased attention as one possible tool to raise an achievable silica dispersion level. Furthermore, due to the increased eco-awareness throughout the world, the request to reduce the emission of volatile organic compounds (VOCs) originating from the production process is another topic drawing more interest. Si 363 (Evonik) and NXT-Z series (Momentive) are the examples of the already commercially 
available mercapto-silanes which were developed in order to fulfill the above described requirements.

The molecular structure of Si 363 is shown in Figure 2-25.[41] On average, two of the alkoxy groups on a silicon atom are substituted by long alkylpolyethers, thus one ethoxy group remains on the silicon atom. The polar part in the longer amphiphilic substituent guarantees a fast adsorption onto the silica surface via hydrogen bonding, which leads to a faster silanization reaction.[23] The apolar part in the substituent works to shield free silanol groups after the adsorption, which also increases the hydrophobation effect by the silane. This long-chain substituents give also a certain steric hindrance effect for the mercaptogroup. For this, the reaction of the mercapto group with the accelerator and the sulfur, which has been suggested as one of the main mechanism for the short scorch time in mercapto-silane system (will be described in Figure 2-30), can be slowed down.

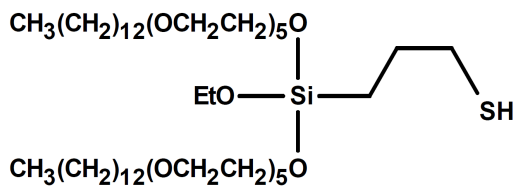

Figure 2-25: molecular structure of VP Si 363[41]

It has been reported that Si 363 enables a reduction of $\tan \delta$ at $60^{\circ} \mathrm{C}$ by more than $40 \%$ (Figure 2-26), of the rolling resistance by more than $10 \%$, and of the emission of VOCs by up to $80 \% .[42-44]$

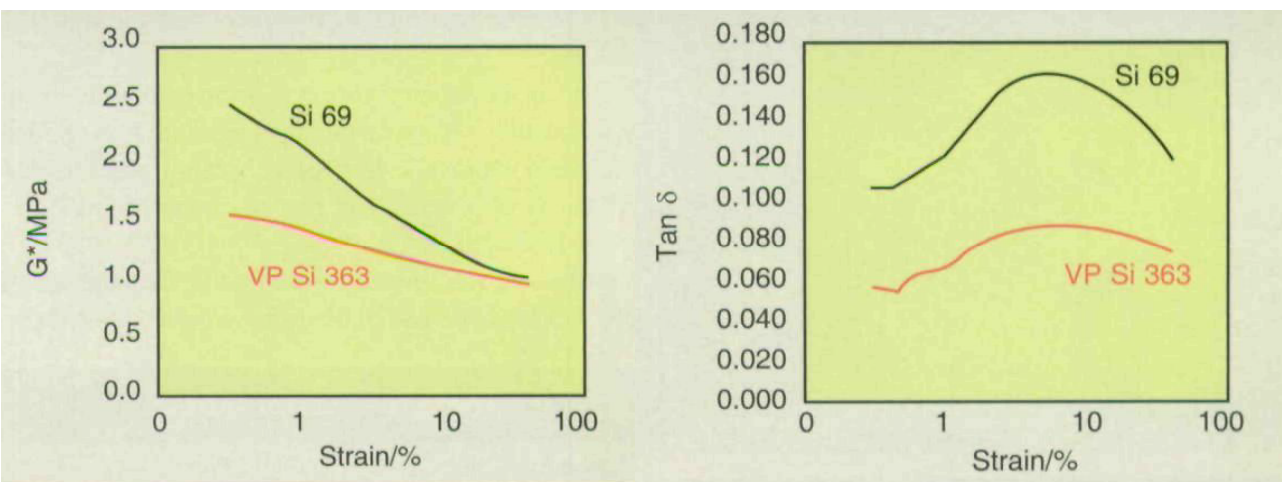

Figure 2-26: RPA curves $\left(60^{\circ} \mathrm{C}, 1.6 \mathrm{~Hz}, \mathrm{G} *\right.$ and $\left.\tan \delta\right)$ of vulcanizates (same silica content for both silane systems) [42] 
The structure of NXT-Z 45 is schematically shown in Figure 2-27,[45] which is an oligomeric combination of mercapto and thiocarboxylate functional silanes. The silicone end of the molecule consists of silicone atoms bridged through non-volatile diols. Since these diols remain in the compound after mixing, almost no VOCs are detected. The octanoyl-blocking group is removed during the final mixing stage by the reaction with DPG (diphenyl guanidine) as described in Figure 2-28, the additionally formed mercapto group contributes to an increase in the reinforcing effect.[46]

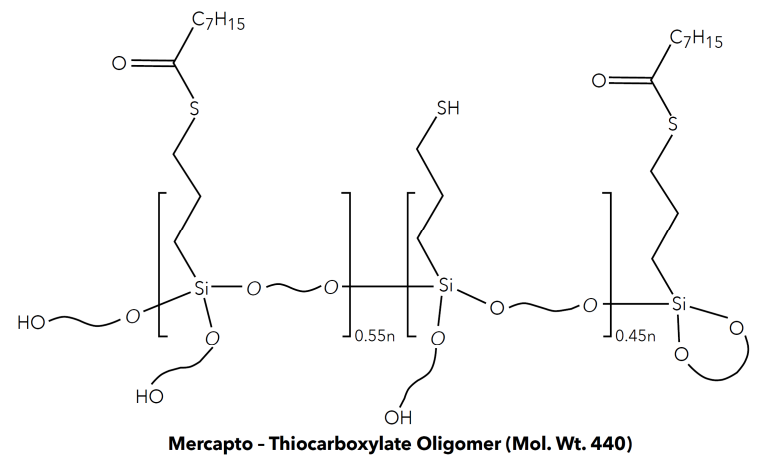

Figure 2-27: molecular structure of NXT-Z 45 [45]

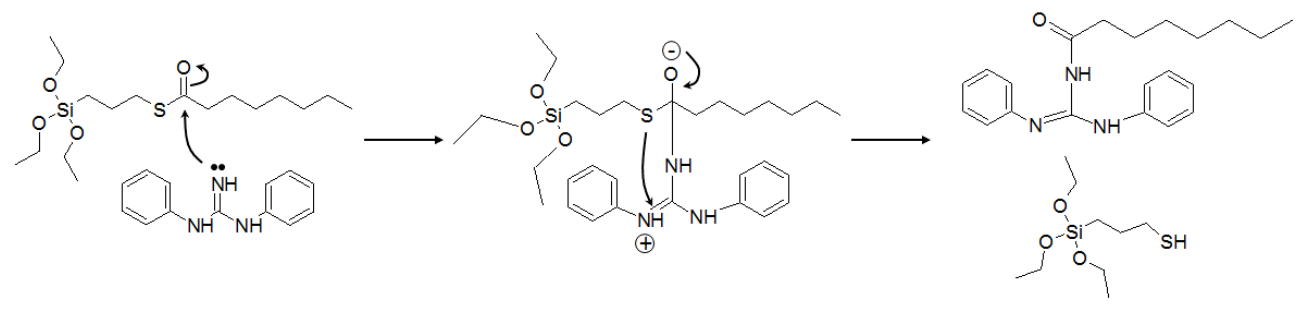

Figure 2-28: Decoupling mechanism of octanoyl-blocking group by DPG [46]

NXT-Z 45 can decrease the Payne effect by $30 \%$ and decrease tan $\delta$ at $60^{\circ} \mathrm{C}$ significantly compared to the conventional $\mathrm{S}_{2}$ or $\mathrm{S}_{4}$ silanes (Figure 2-29).[47]
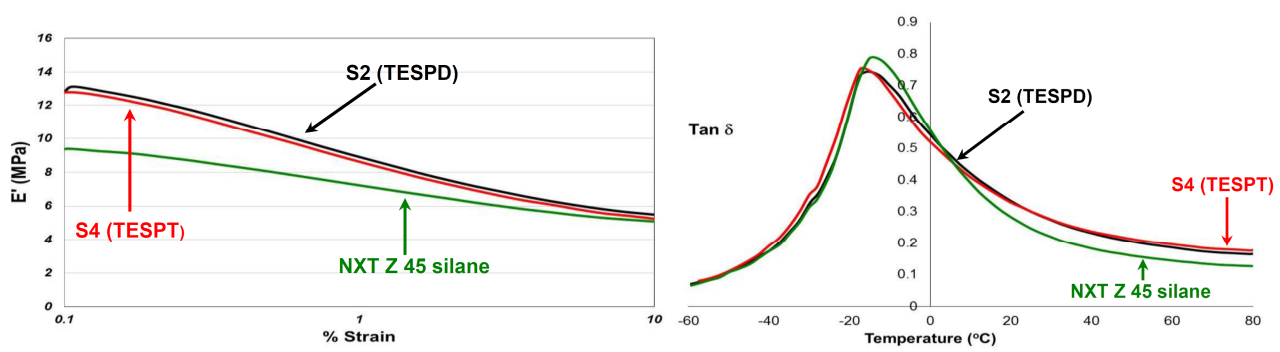

Figure 2-29: Payne effect and tan $\delta$ curve (same silica content for both silane systems) 
However, it has been reported that mercapto-silane compounds tend to show a shorter scorch time by using the same curative package as in the Si 69 system. $[42,47,48]$ The following mechanism has been proposed to explain this behavior (Figure 2-30).[48] The mercapto-silane which has already coupled to the silanol group on the silica surface reacts with the accelerator CBS which is added during the final mixing stage. This results in the formation of an intermediate-1 as depicted in Figure 2-30. When an amine like DPG and a sulfur are also present in the system, the intermediate-1 incorporates sulfur easily to create the highly active intermediate-2, which can react quickly with the polymer. This mechanism leads to a shorter scorch time as observed for the mercapto-silane containing rubber compounds. Additionally, this reaction mechanism might also be the reason for the higher reinforcement effect in mercapto-silane rubber compounds. As a countermeasure for this too fast cure rate, the use of TBzTD with reducing or even eliminating DPG is normally recommended.[42,47]
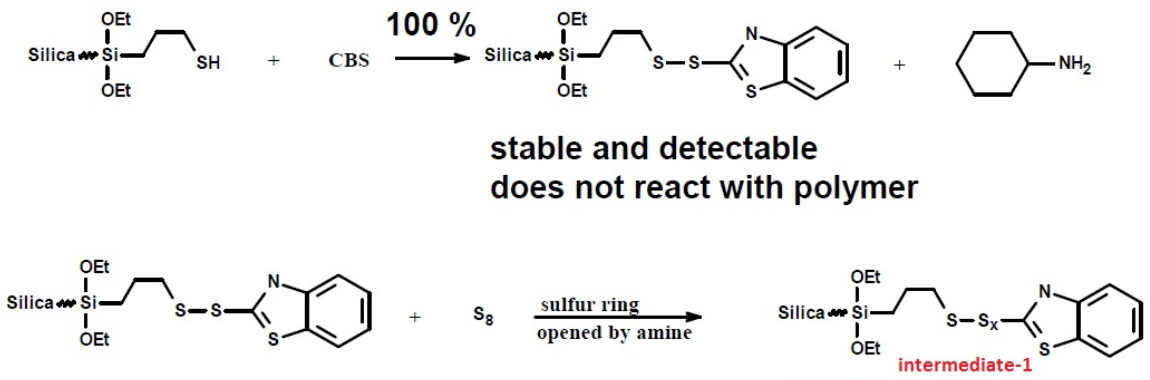

highly reactive

does react with polymer

quantitatively

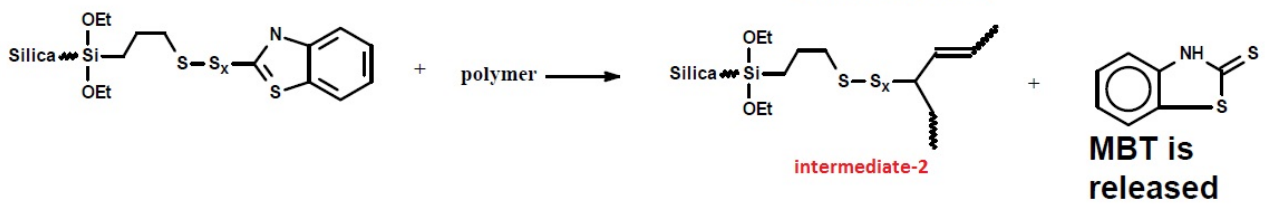

Figure 2-30: Reaction mechanism of mercapto-silane with rubber in the presence of curatives [48]

However, it is frequently observed that the mercapto-silane rubber compounds have a worse dump appearance even after the first mixing stage, together with a high Mooney viscosity. These phenomena are observed in the compounds which do not contain any curatives, therefore, this cannot be explained by the above described scorch mechanism 
(Figure 2-30). There is a report that demonstrates adding $\mathrm{ZnO}$ and stearic-acid at the 1st mixing stage is better for obtaining lower Mooney viscosity than adding them at the final mixing stage.[43] However, the mechanism has not been clarified yet.

\section{2-4 Rubber reinforcement}

Reinforcement of rubber by fillers strongly depends on the interaction between the rubber and the filler as well as the dispersion level of the filler in the rubber matrix. Strain dependency of the modulus and bound rubber are used to describe the reinforcing behavior.

\section{2-4-1 Payne effect}

Above a critical filler concentration, which is called the percolation threshold, the properties of the reinforced rubber material change drastically, because a filler-filler network is established. The continuous disruption and restoration of this filler network upon deformation is well visible in the Payne-effect, as represented in Figure 2-31. It illustrates the strain-dependence and the strain-independent contributions to the complex shear or tensile moduli for filled compounds.
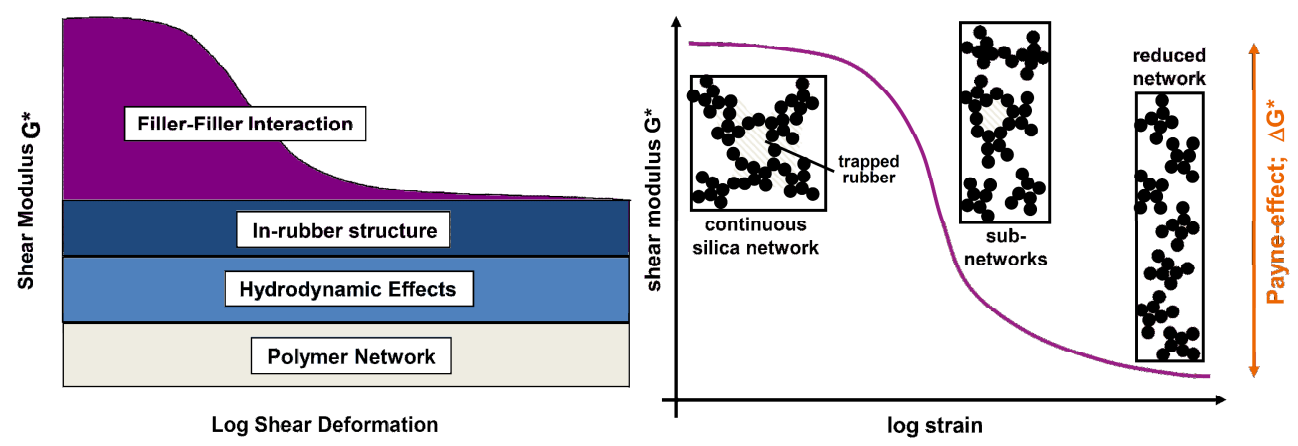

Figure 2-31: The Payne effect concept of reinforcing filler-filled rubber compounds [49-52]

The main contributions to the complex modulus are:

1. the strain-independent contribution of the polymer network, as commonly represented by polymer network theories to be proportional to the network chain density or cross-link density 
2. the strain-independent hydrodynamic effect, due to the mechanical obstruction to deformation by the presence of (spherical) particles in the polymer matrix

3. the strain-independent filler-polymer interaction or in-rubber structure, where the filler particle acts as poly-functional cross-link site

4. the strain dependent filler-filler interaction: the Payne effect

In the very small deformation range, the filler-filler interaction which leads to a much higher modulus than the virgin rubber can withstand the deformation. With increasing strain, the filler-filler network which is constituted of physical bonds such as van der Waals forces and hydrogen bonds is partly and finally completely broken down. Hence, the polymer matrix starts to bear the deformation alone resulting in the lower modulus. Practically, the $\Delta \mathrm{G}^{\prime}$ value calculated as the difference between the $\mathrm{G}^{\prime}$ value at very small strain and the value at a large deformation range is widely used as an indicator for the fillerfiller interaction.

\section{2-4-2 Filler - Polymer interaction}

The filler - polymer interaction in the silica / silane filled compounds is very different from that one in the CB filled compounds. The bound rubber measurement is known as a method to estimate the filler-polymer interaction. When an uncured filled compound is extracted by a solvent which is able to dissolve the rubber (mainly toluene is used), the remaining rubber parts which are attached to the filler are called "bound rubber". The bound rubber formed by carbon black has been considered to be of dominantly physical adsorption nature. Therefore, it was reported that the amount of bound rubber decreases at temperatures above $80^{\circ} \mathrm{C}$ because of the increased molecular mobility of the rubber.[53]

Several bound rubber models have been proposed by many researchers, as shown in Figure 2-32. In the occluded rubber model, during self-association in the rubber matrix, filler clusters trap some part of rubber inside their structure (Figure 2-32 (a)). In the shell rubber model, rubber exists on the filler surface resulting from chemical adsorption on the filler surface (Figure 2-32 (b)). O'Brien proposed the advanced shell rubber model, where the polymer on the carbon black surface consists of two different layers which are a glass-state layer and a rubber-state layer (Figure 2-32 (c)). 


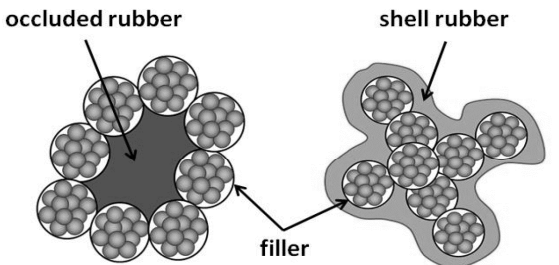

(a)

(b)

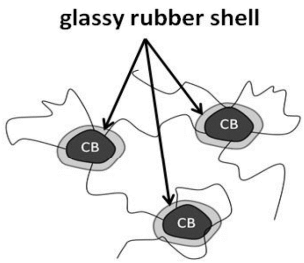

(c)

Figure 2-32: Bound rubber models; (a) occluded rubber model [54,55], (b) shell rubber model [56,57], (c) glassy rubber shell model [58]

In the silica - silane / rubber compounds, the presence of the rubber layer chemically bonded on the silica surface has been confirmed.[59] The chemically bonded bound rubber can be detected by applying the ammonia treatment for the bound rubber sample. When the bound rubber sample is immersed in toluene again but this time under an ammonia atmosphere, the physical interaction inside the sample can be removed, which results in only remaining chemically bonded rubber. One example of this is shown in Figure 2-33. The curve in the left figure which is described as "untreated with $\mathrm{NH}_{3}$ " represents the overall bound rubber content, and the other curve in the same figure which is described as "treated with $\mathrm{NH}_{3}{ }^{\prime \prime}$ correspond to the chemically bounded rubber content. The difference between the overall and the chemically bound rubber content is expressed as physically bound rubber content in the right figure. In the samples which are the natural rubber / silica / TESPT compounds, the amount of chemical bound rubber is higher than that of physical bound rubber.[38]
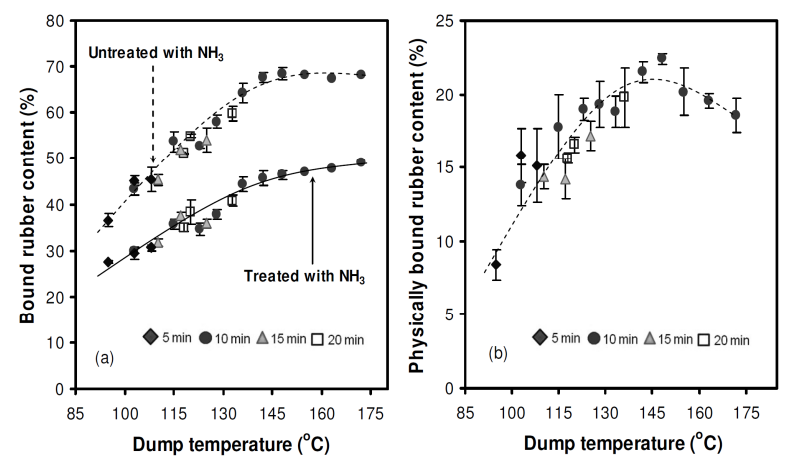

Figure 2-33: Bound rubber contents of silica - filled NR compounds before and after ammonia treatment [38] 
The silica / silane reinforcement model was proposed by Luginsland et al. based on the hydrodynamic-occlusion-interaction theory according to Medalia, as shown in Figure 234.[52] The compound contains immobilized rubber on the silica surface by chemical bonding. Even by using silane coupling agents about $75 \%$ of the silanol groups are left over.[13] Therefore, the silica aggregates can still form a network due to the high selfcohesive forces on the surface together with the large polarity difference between rubber and silica. A certain amount of rubber can be trapped during this process. Furthermore, there is also loosely adsorbed rubber on the silica surface, though the interaction is very weak in this case. During the deformation of the compound, some parts of the occluded rubber which are not attached to the rubber by physical interlocking or Van-der Waals forces can be released due to a breakdown of the silica network. As a result, the amount of deformable rubber is increased due to this released rubber. But the chemically bonded rubber remains immobilized on the surface and thus behaves as a stiff filler, which therefore influences the modulus even under high strains. This effect is defined as "in-rubber structure".[52]

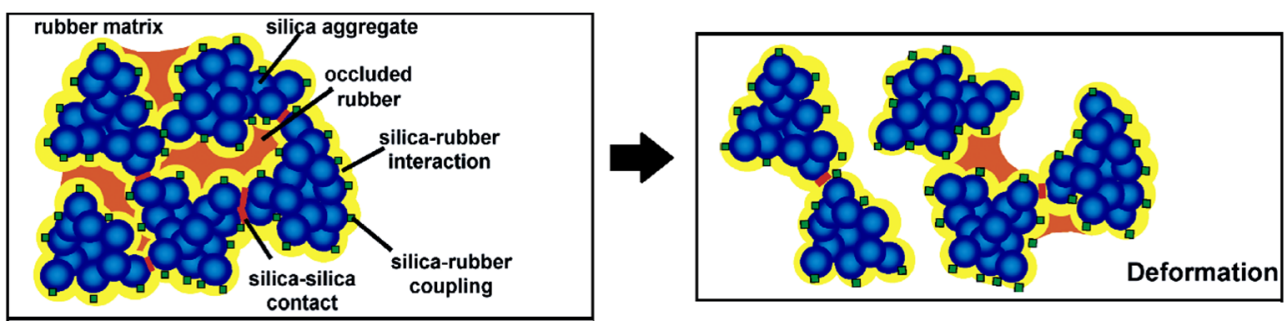

Figure 2-34: Proposed simple model of silica/silane reinforcement [52]

(a) No deformation, (b) after large deformation

In has been reported in the literature that the chemical bound rubber significantly affects the viscoelasticity of the compound.[22,38] Mihara defined a "specific bound rubber" content which is the ratio of the bound rubber content after the ammonia treatment and the CTAB value of the used silica (equation 3 ). He reported a good correlation between this content and $\mathrm{G}^{\prime}$ at $0.56 \%$ strain or $\tan \delta$ at $60^{\circ} \mathrm{C}$ respectively (Figure 2-35). Both in-rubber properties decrease with increasing specific bound rubber content. It has been reported in the literature that the value of $\tan \delta$ at $60^{\circ} \mathrm{C}$ correlates with the inter-aggregate distance of

$$
\text { Specific bound rubber }=\frac{\text { Bound rubber after ammonia treatment }}{\text { CTAB surface area of silica }}\left[\mathrm{g} / \mathrm{m}^{2}\right]
$$


the silica.[60] Therefore, Mihara also concluded that the increased specific bound rubber extended the inter-aggregate distance resulting in the lower tan $\delta$ values.
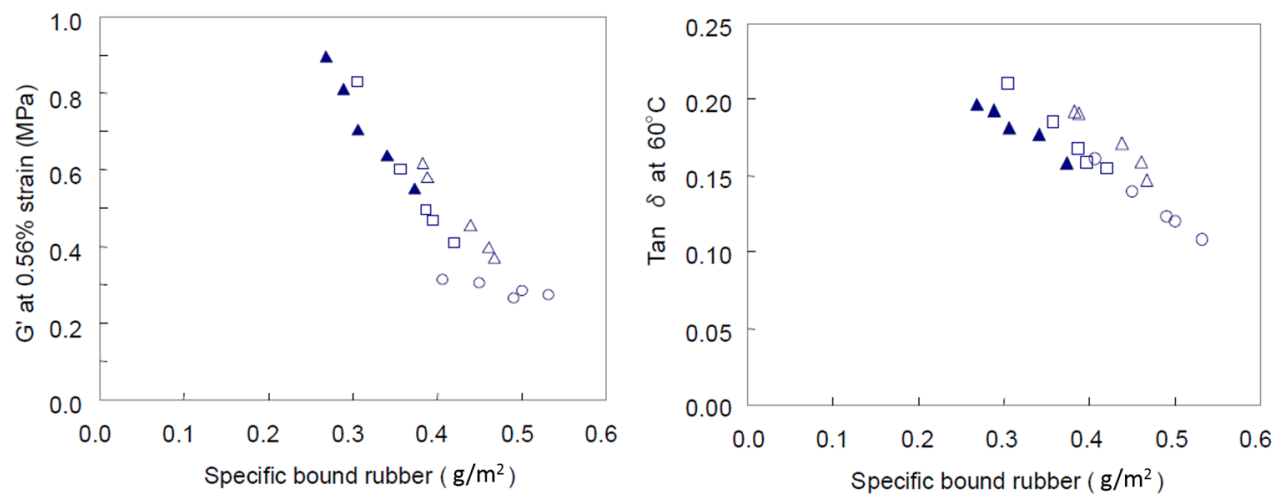

Figure 2-35: The relationship between the specific bound rubber and $\mathrm{G}^{\prime}$ at $0.56 \%$ strain or $\tan \delta$ at $60{ }^{\circ} \mathrm{C}$ of the various types of silica filled SBR / BR compounds; (o) Zeosil $1115 \mathrm{MP}$, ( $\Delta$ ) Zeosil 1165 MP, (口) ULTRASIL 7005, (ム) ULTRASIL VN3 [22]

\section{2-4-3 Silica flocculation}

It is known from many literature citations that silica aggregates which were dispersed in the rubber matrix during the mixing process tend to re-agglomerate during the storage period and the vulcanization step because of the large polarity difference between rubber and silica. This results in an increment of the complex modulus.[61,62,37] This reagglomeration process is named flocculation process and influence he final dispersion level of the silica in the vulcanized rubber. This flocculation behavior strongly depends on temperature. The activation energy was reported to be less than $10 \mathrm{~kJ} / \mathrm{mol}$ which means that the flocculation process is considered as a mainly physical phenomena.[62] The chemical bound rubber decreases the polarity on the silica surface by shielding it and therefore leads to the suppression of the re-agglomeration process (Figure 2-36).[62] 


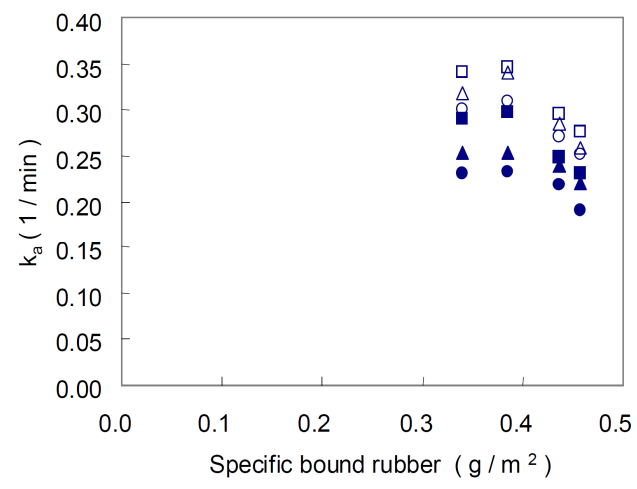

Figure 2-36: Flocculation rate constant $\mathrm{k}_{\mathrm{a}}$ as a function of specific bound rubber at different measurement temperatures; (•) $90^{\circ} \mathrm{C},(\Delta) 100{ }^{\circ} \mathrm{C},(\square) 110^{\circ} \mathrm{C},(\bigcirc) 120^{\circ} \mathrm{C},(\triangle) 130{ }^{\circ} \mathrm{C},(\square)$ $140{ }^{\circ} \mathrm{C}[62]$

\subsection{Vulcanization}

Even when the usage of silica becomes mainstream for tire tread compounds instead of $\mathrm{CB}$ as a reinforcing filler, there is no change that the vulcanization is one of the most important key steps to produce high-quality tires. Vulcanization is fundamentally a set of chemical reactions whereby single polymer chains (plastic in character) are chemically linked into a three-dimensional network having tough elastic properties. This transformation from a soft, plastic material to a tough, elastic material is the basis for the engineering properties of vulcanized rubber.

Despite the fact that it has past more than 150 years since the sulfur vulcanization was discovered, its exact mechanism is still not completely clarified. The reason for this is that, during the vulcanization, a very small percentage of material reacts with the polymer transforming it into a network of intractable material that is difficult to analyze by traditional methods. Much of the understanding has been developed through model compound studies, investigations of vulcanization reaction kinetics and tracing the amount and reaction products of the accelerator and sulfur chemicals.

As mentioned in the previous section 2-3-5, it has been considered that the poly-sulfide type of silane coupling agents such as TESPT release elementary sulfur from the molecule at a sufficient temperature, which leads to a certain amount of vulcanization reaction even at the $1^{\text {st }}$ mixing stage without curatives. The consequence of it is a premature scorch and 
a high Mooney viscosity resulting in worsening processability. With this point in mind, the vulcanization mechanism without any accelerator, namely the unaccelerated sulfur vulcanization, is summarized first followed by the generally accepted accelerated sulfur vulcanization mechanism. Lastly, some example of model compound studies, especially using olefins as model compounds are introduced.

\section{2-5-1 Unaccelerated sulfur vulcanization}

Because of the simplicity of sulfur-only formulations, the mechanism of unaccelerated sulfur vulcanization seems to be easy understandable. However, the reverse is actually true. Many research studies have found that there are several reactions involved in the mechanism, such as double bond migration, isomerization and saturation to chain cleavage, cyclization and formation of vicinal crosslinks. $[63,64,65,66,67]$

A major focus point was to clarify if the nature of the reaction mechanism is polar (ionic), radical or following a mixed mechanism arising from the possible reactions of elemental sulfur. The $\mathrm{S}_{8}$ ring is capable of undergoing homolytic (radical) and heterolytic (polar / ionic) ring opening reactions.

The proposed radical mechanism of NR starts from a homolytic scission of the octet sulfur ring as shown in Figure 2-37.[63] The sulfur radical abstracts a proton from the rubber to form a carbon radical on the elastomer. The elastomer radical then ring-opens another $\mathrm{S}_{8}$ ring to form a rubber-bound sulfur radical capable of forming a crosslink structure. The coupling of this rubber-bound sulfur radical to another double bond results in the alkenylalkyl product with maintaining cis structure in the alkenyl part. However, the carbon elastomer radical can be transformed to a tertiary radical. If sulfur couples to the tertiary radical, the resulting crosslink product has a trans structure with a migration of the double bond, as shown in Figure 2-38.[63] 

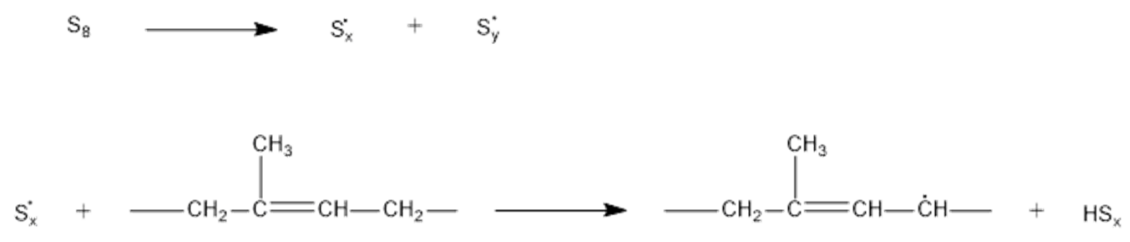<smiles>CCC(C)=C[CH-][C+]CC(C)=CC(C)C</smiles><smiles>CCC=C(C)C[C-]C(S)C=C(C)CC</smiles><smiles>CCC=C(C)CC[O-]</smiles><smiles>CCC=CC[CH-]CC(C)(CC)SC(C)C=C(C)CC</smiles>

Figure 2-37: Proposed radical mechanism for unaccelerated sulfur vulcanization 

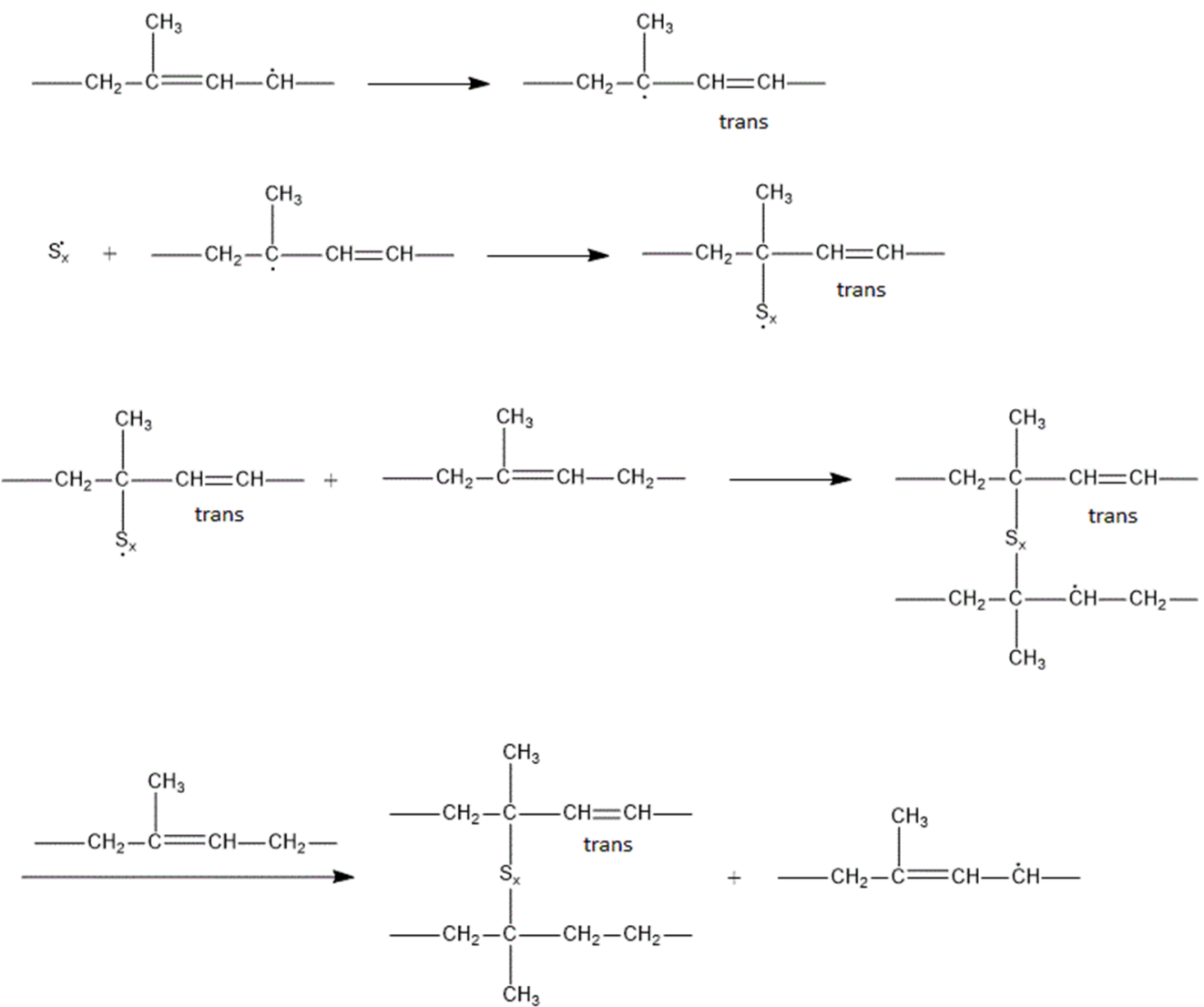

Figure 2-38: Proposed radical mechanism for isomerization and double bond migration in sulfur-only vulcanization

The key step for the ionic unaccelerated sulfur vulcanization mechanism is the formation of the cyclic sulfur-carbon charged ring (Figure 2-39). For this mechanism isomerization occurs through the nonsulfurated rubber ion; when this ion goes back to the elastomeric repeat unit, the olefinic moiety, can form in either the cis or trans configuration. The formation of cyclic structures can also occur. The sulfur crosslink can cleave at the relatively labile S-S bond; the sulfur chain then reacts intramolecularly to form a cyclic structure (Figure 2-40).[66] 


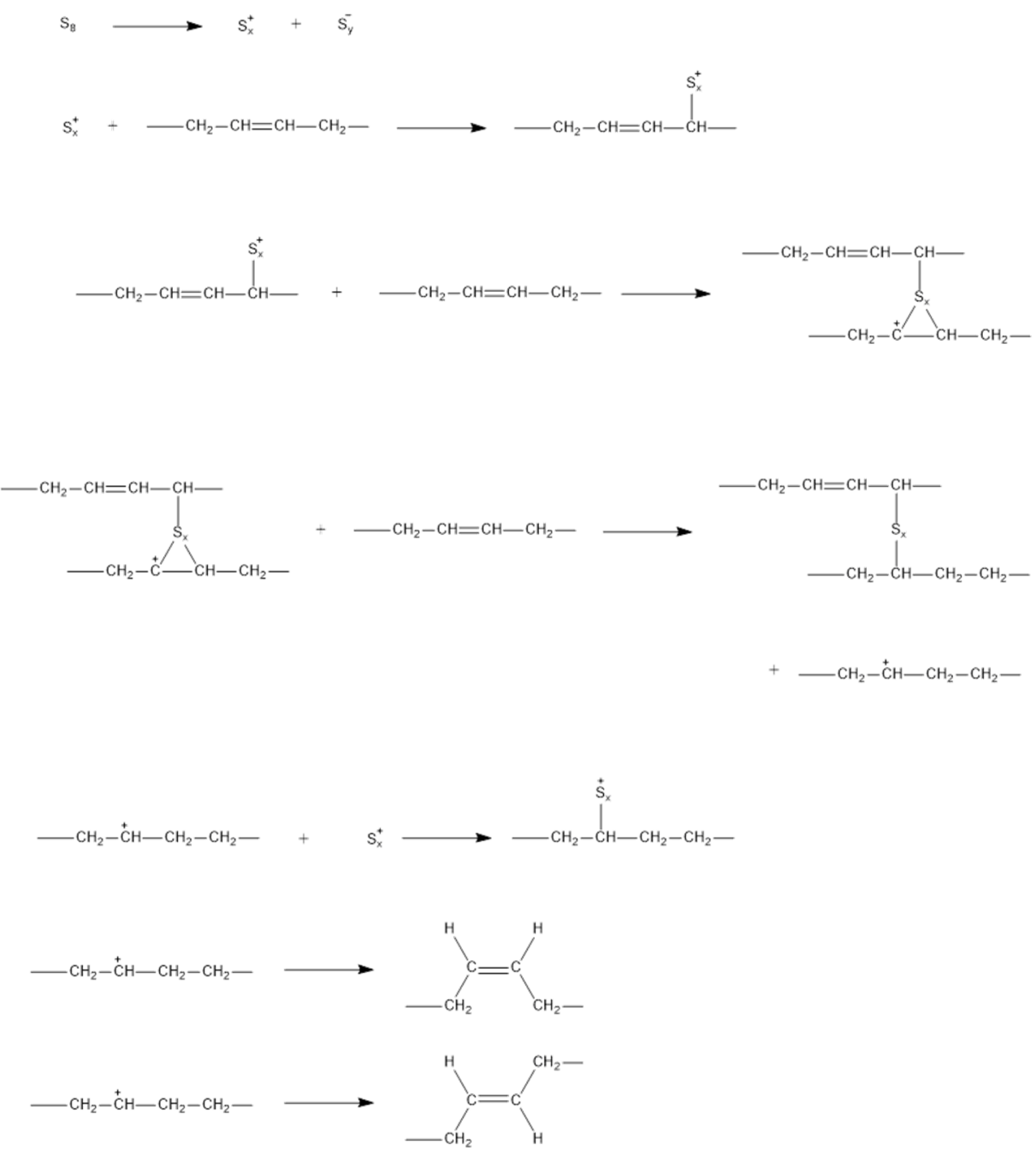

Figure 2-39: Proposed polar mechanism for unaccelerated sulfur vulcanization

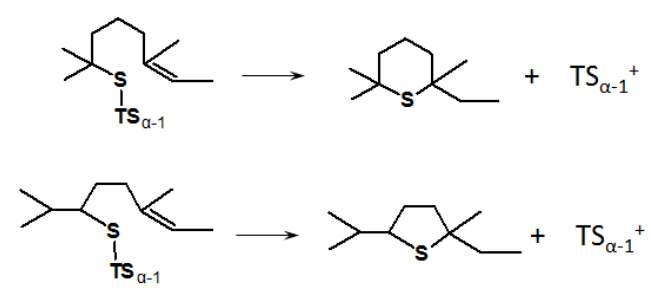

Figure 2-40: Mechanism of cyclic formation for polar mechanism of unaccelerated sulfur vulcanization 
The studies using solid-state ${ }^{13} \mathrm{C}$ NMR characterize the network structure resulting from unaccelerated sulfur vulcanization of NR and BR, as shown in Figure 2-41. In the case of NR system, although NR has three allylic positions, the structures where sulfur couples to all three allylic carbons respectively as well as added to the double bond were confirmed. Cyclic structures and cis-trans isomerization were also detected, but alkenyl structures were the main components.[63-65]
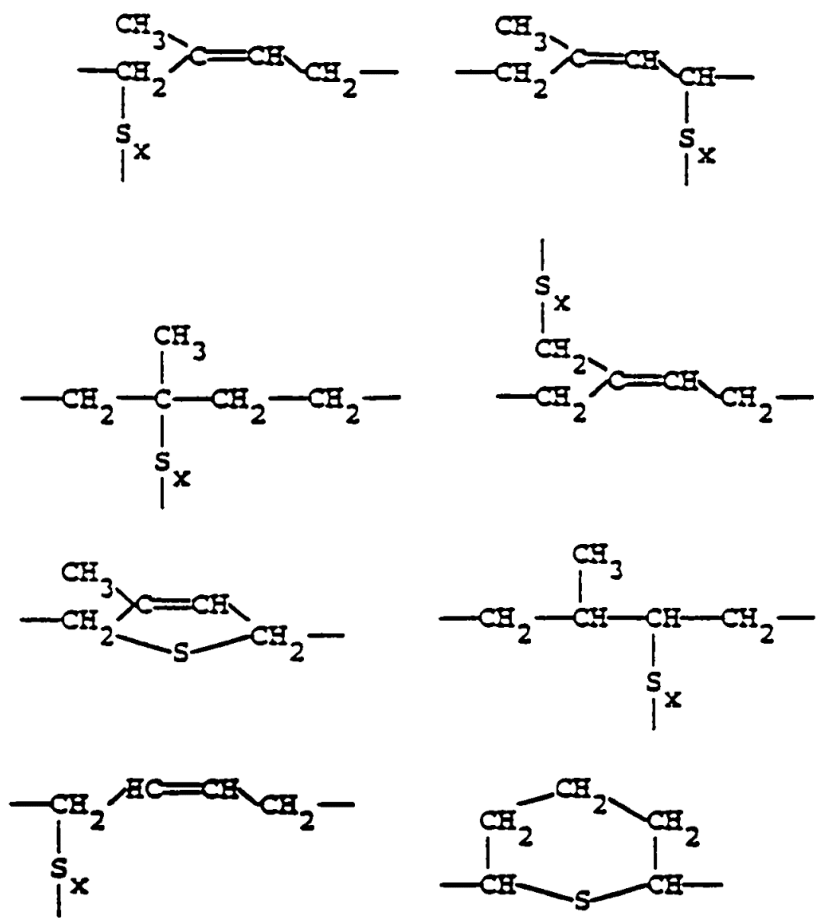

Figure 2-41: Network structures found in unaccelerated-sulfur NR and BR vulcanizates

It was found that $\mathrm{ZnO}$, combined with stearic acid, reduces the vulcanization time and improves the in-rubber properties, even in the case of unaccelerated vulcanization.

\section{2-5-2 Accelerated sulfur vulcanization}

Accelerated vulcanization gives both improved crosslinking efficiencies and rates. Figure 2-42 and Figure 2-43 show the generally accepted overall scheme for the accelerated sulfur vulcanization.[68,69] 


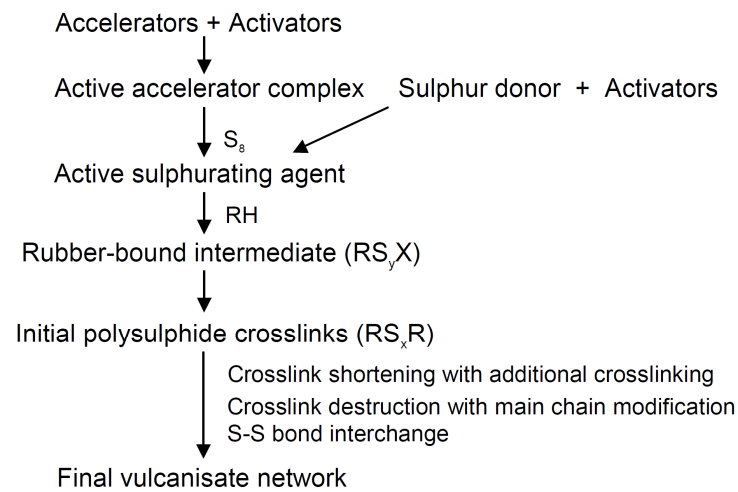

Figure 2-42: Generalized mechanism of accelerated sulfur vulcanization by Morrison et al.; $\mathrm{R}=$ rubber chain, $\mathrm{H}=$ allylic proton, and $\mathrm{X}=$ accelerator residue [68]

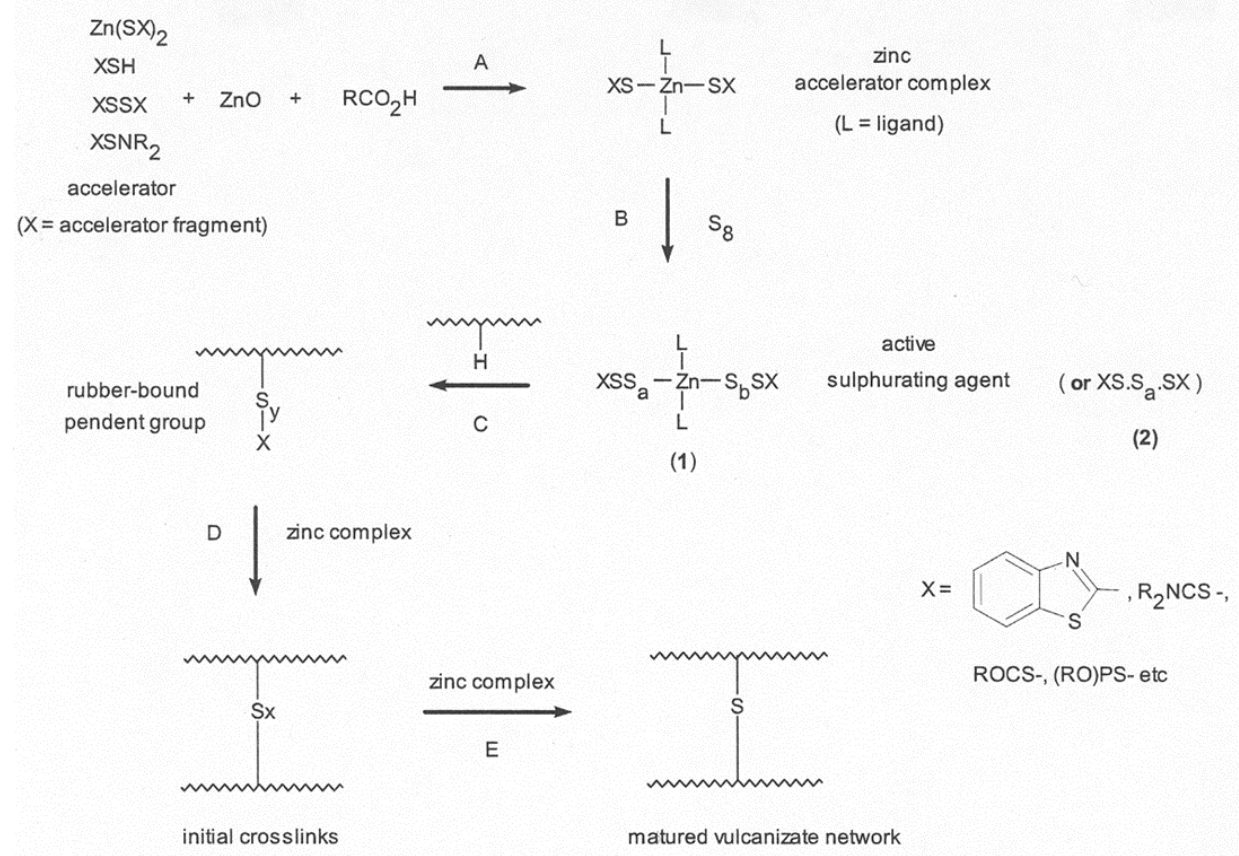

Figure 2-43: Scheme of accelerated sulfur vulcanization proposed by L. Bateman et al. [69]

These suggest the formation of an active accelerator complex via a reaction between the activator and the accelerator as a first step in the vulcanization process. These complexes interact with sulfur, a sulfur donor or other activators to generate the active sulfurating 
agent. It has been suggested from many different studies that $\mathrm{Zn}^{2+}$-ions form these active complexes with accelerators, which are more reactive than the free accelerator.[63,70] These activated species then react with the allylic sites of the rubber chain and become intermediates or crosslink precursors, which are accelerator-terminated polysulfidic pendant groups attached to the rubber chain. These intermediates react with other intermediates or polymer chains resulting in polysulfidic crosslinks.

\section{2-5-3 Model compound vulcanization}

As mentioned before, standard chemical analytical methods such as NMR, LC or GC-MS are difficult to apply to analyze vulcanized rubber, because of the insolubility of the elastomer network, the low concentrations and the variety of cross-linked structures. For this reason, Model Compound Vulcanization (MCV), the vulcanization of a low-molecular weight model for the rubber polymer, has been used widely to elucidate the vulcanization kinetics and mechanisms. The vulcanization process is simulated by the reaction of a low molecular weight model compound such as olefin with curatives. The generated products are then analyzed by different chemical analytical methods which can be carried out more easily compared to vulcanized rubber.

The model olefin which has been used as an alternative for NR is 2-methyl-2-pentene $(2 \mathrm{~m} 2 \mathrm{p})$. The main cross-linked structure of this molecule in unaccelerated sulfur vulcanization (Figure 2-43) has alkenyl-alkyl structures.[63,71] While, di-alkenyl structures were reported as main reaction products with the indicated ratio in Figure 2-44 in the case of CBS accelerated sulfur vulcanization in the model system.[72] Furthermore, several studies to investigate the influence of cure time, type of accelerator and the presence of filler and additives on the distribution between mono, di and tri sulfidic cross-links of $2 \mathrm{~m} 2 \mathrm{p}$ were carried out. When the total yield of cross-linked products obtained in the model studies were compared with the $300 \%$ modulus of the actual cured NR compounds, they showed a linear correlation, which indicates a similar reactivity of the MCV model and rubber. This underlines the usefulness of MCV as a vulcanization research technique. 


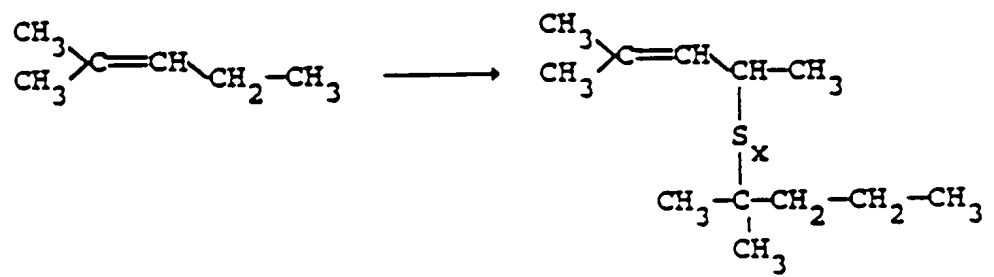

Figure 2-43: Main cross-linking structure found in 2-methyl-2-pentene unaccelerated sulfur vulcanization
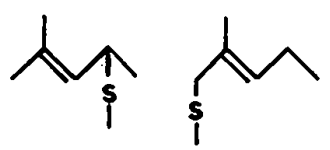

II

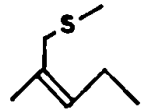

III

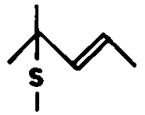

IV

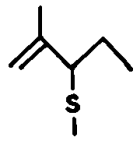

V

$50-65 \%$ $35-45 \%$ $<5 \%$ $<7 \%$

Figure 2-44: Cross-linking structures with the ratio found in 2-methyl-2-pentene accelerated sulfur vulcanization

Cis-3-hexene, cyclohexene, cis,cis-1,5-cyclooctadiene are the representatives which have been used as model substances for BR. $[67,72,73]$ Using cis-3-hexene in the CBS accelerated sulfur vulcanization, bis-alkenyl crosslinks were formed by $\alpha$-substitution of an allylic hydrogen by a sulfur bridge, as shown in Figure 2-45. It was concluded that, apart from $\alpha$ substitution, also substantial allylic rearrangements has taken place ( $\alpha$-substitution accompanied by a shift of the double bond, leading to the structures 2 and 3 . Only small amounts of products 4 and 5 , resulting from addition reaction, were present in the reaction mixture.

Cis-1-phenyl-3-heptene and cis-1,7-diphenyl-3-heptene were used for SBR model substances in comparison with BR model chemicals such as cis-3-heptene, trans-4-octene or cyclooctene to study the CBS accelerated sulfur vulcanization including ZnO.[74] Under the vulcanization condition at $150^{\circ} \mathrm{C}$ for 10 minutes, all BR model compounds produced the cross-linked products resulting from $\alpha$-substitution. However, even after 30 minutes of heating, SBR model substances didn't show any vulcanization products. Even when the more reactive accelerator TMTD was used and the reaction time was prolonged to 60 
minutes, only cis-1-phenyl-3-heptene underwent vulcanization. This clearly indicates that the sulfur bridge is hampered near phenyl groups. Therefore, it was concluded that, in the case of SBR, only the BR part participates in the vulcanization process.

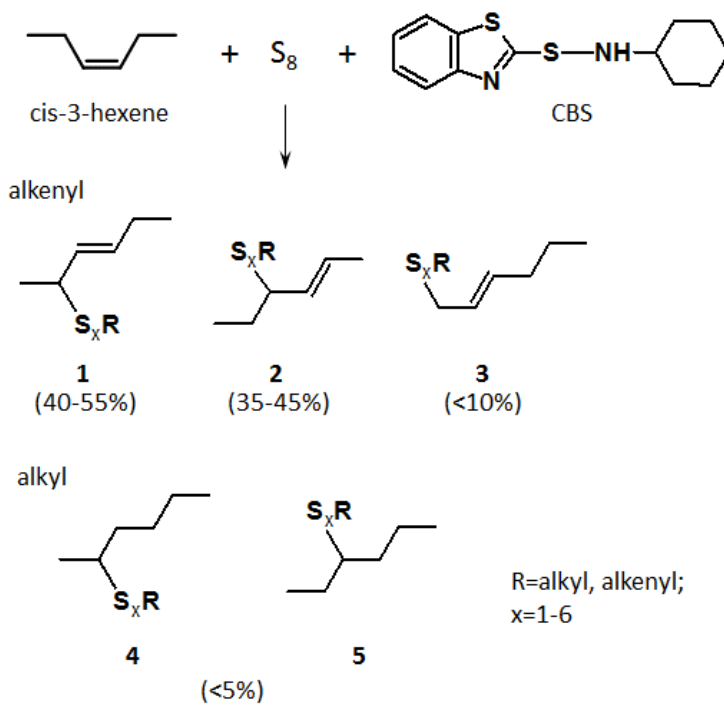

Figure 2-45: Reaction products from the CBS accelerated sulfur vulcanization of cis-3hexene

Versloot et al. investigated the isomerization mechanism during vulcanization by using different structures of olefins shown in Figure 2-46. The sulfur vulcanization of all investigated olefins in the presence of zinc oxide and TMTD resulted in the formation of bis(alkenyl) sulfides with sulfur attached to the allylic carbons. The driving force of the isomerization was the stability of the resulting double bond structure after the vulcanization, which was determined by the number of alkyl substituents. An increase of the number of alkyl substituents results in more stable alkenes because of the inductive effect of alkyl groups. In the case of TME, since the isomerized structure is unstable, a rearrangement of the double bond only took place for less than $5 \%$. In contrast to this, the vulcanization of 1 decene results in a product mixture in which a complete isomerization of the double bond had occurred, i.e. leading to the more stable bis(2-decen-1-yl) sulfides. The vulcanization of trans-3-hexene resulted in a rearrangement of the double bond, leading to an almost 1:1 mixture of 3-hexen-2-yl sulfides and 2-hexen-4-yl sulfides, which did not significantly differ 
in their stability. For the study with 2-hexene similar products were observed, but a third structure was additionally identified, i.e. 2-hexen-1-yl sulfides (Figure 2-46).[40,75]

Model olefin bis(alkenyl)sulfides (arrows indicate the binding sites of S)

Figure 2-46: Experimental composition of the vulcanizate for different model olefins [40]

\section{2-6 Motivation and Focus of the project}

The introduction of the "green tire technology" in 1992 brought a significant improvement on tire performances. It has passed more than 25 years since then, and nowadays, a lot of tire companies produce the full-silica filled compounds for the passenger car tire tread. During this period, the mechanism of the silica / silane technology has been clarified to a great extent.

However, the requirements for the low fuel consumption tire from the global environmental protection point of view and improvement of the safety level are constantly increasing. Therefore, the further advances in the technology are necessary to provide a higher performance level of tires. 
The usage of higher surface area silica seems to have a possibility to reach a higher balanced level of tires with regard to rolling resistance, wet grip and abrasion resistance because of the high reinforcing potential of it. However, generally, it is quite difficult to disperse polar silica clusters into a non-polar rubber due to the low compatibility between the rubber and the silica as well as the strong self-cohesive forces between single silica clusters. This tendency increases with increasing surface area of silica. To overcome this problem, the use of mercapto-silanes has become more and more into focus over the last years. Although there are some commercially available mercapto-silanes such as Si 363 and NXT-Z series, in addition to those silanes, even the usage of the triethoxy type of mercaptosilane enables an increase of the silica dispersion level compared to the conventional triethoxy type of sulfide-silanes. However, the usage of mercapto-silanes is apt to lead to processing difficulties. But the mechanisms which enable to increase the silica dispersion level but to cause also the worse processability have not been clarified yet.

In general, increasing the silanization reaction efficiency can lead to a better dispersion of silica. However, it has been reported that as long as the type of alkoxy group is the same for silanes like an ethoxy group, the silanization reaction rate of mercapto, di-sulfide or tetra-sulfide silanes are almost the same (See section 2-3-3). Hence, the obtained improvement of the silica dispersion level using mercapto-silanes cannot be explained in terms of the silanization rate. This implies that the reaction of the sulfur function of silane with the rubber seems to have the main influence on the achievable silica dispersion level as well as the processability.

It has been generally considered that the silane - polymer reaction for sulfide silane systems occurs mainly during the vulcanization stage. However, there are reports investigating the reactivity of sulfide silanes which show that the chemically bound rubber is also formed in the non-productive mixing stage and affects the values of tan $\delta$ at $60^{\circ} \mathrm{C}$ as well as those of the Payne effect (See section 2-4-2). Therefore, these points have to be investigated also in the mercapto-silane systems in order to understand if there are principle differences compared to the sulfide silane system.

Based on the above mentioned points, one goal of this thesis is the understanding of the reactivity and the reaction mechanism of mercapto-silanes with rubber in comparison with those of sulfide-silanes with rubber, and how these points affect the physical properties of rubber compounds. Furthermore, based on the gained knowledge, a further goal is to find 
possibilities to overcome the observed processing difficulties without losing in-rubber performance.

This study was started with a model study using low molecular weight compounds instead of rubber, followed by a silica filled rubber compound study. The effectiveness of the model compound study, which uses low molecular weight chemicals instead of rubber, has been widely confirmed in the vulcanization reaction study (see section 2-5-3). The vulcanization follows complicated reaction schemes where many different chemicals are involved which results in a variety of reaction products. However, the model study simplify the system and also increase the range of applicable analytical methods, which helps to understand the mechanism step by step. For the same reason, this method can be considered as an effective way to understand deeply the rubber / silane reaction.

Furthermore, the type of rubber for tire tread compounds is normally chosen depending on the tire category accompanying different demand properties. Even in the same classification of rubber such as SBR or BR, there are many variations with regard to the micro structure of the rubber, and these differences have an influence on the reactivity of the rubber with silanes. Therefore, the influence of the double bond structure (1,2-butadiene, 1,4-cis/trans-butadiene, 1,4-cis/trans-isoprene), on the reaction with silanes will be investigated in this model study by using different types of olefins, which have only one double bond in the molecule. Based on the information there, the research will move to the second step of model studies which uses the model compounds having multiple double bonds in a molecule.

And finally, the evaluation of silica filled rubber compounds using the findings in the model studies will be done in order to gain a better understanding of the reinforcing mechanism in both silanes with different rubber systems.

All these investigations should enable to understand the reason for both, the advantage and disadvantage in the mercapto-silane system as well as to find a possibility to bring a better balance in the real rubber system. 


\section{2-7 Reference}

[1] E. Cichomski, "SILICA-SILANE REINFORCED PASSENGER CAR TIRE TREADS", PhD. Thesis: 2015, University of Twente, Enschede, the Netherlands, 2015.

[2] K. Akutagawa, Y. Ozawa, H. Yamada, T. Hamada, Journal of the Society of Rubber Science and Technology, Japan, 80, (2007) 40-47

[3] Y. Ozawa, Koubunshi, 54, (2005) 750-754

[4] K.A. Grosch, Rubber Chem. Technol., 37, (1964) 386-403

[5] N. Amino, Journal of the Society of Rubber Science and Technology, Japan, 88 (2015) 3742

[6] S. Uhrlandt, A. Blume, Rubber World, 228, (2003) 43-45

[7] A. Blume, S. Uhrlandt, Am. Chem. Soc., Rubber Div. Meeting, Dallas, Texas, April (2000)

[8] Elastomer technology course 2015 at University of Twente

[9] R.K. Iler, The chemistry of silica: solubility, polymerization, colloid and surface properties and biochemistry of silica, John Wiley and Sons, New York, (1979)

[10] B. Rodgers, Rubber Compounding - Chemistry and Applications, chapter 7_silica and silanes, Marcel Dekker Inc., New York - Basel (2016)

[11] A. Voet, J.C. Morawski, J.B. Donnet, Rubber Chem. Technol., 50, (1977) 342-355

[12] F. Grunert, A. Wehmeier, W.K. Dierkes, A. Blume, International Elastomer Conference, Cleveland, Ohio, October 9-12, (2017)

[13] A. Blume, M. El-Roz, F. Thibault-Starzyk, Kautsch. Gummi Kunstst., 10 (2013) 63-70

[14] A. Blume, M. Janik, J.-P. Hanau Gallas, F. Thibaultbault-Starzyk, A. Vimont, Kautsch. Gummi Kunstst., 61 (2008) 359-362

[15] Legrand AP (ed.). The Surface Properties of Silicas. John Willey \& Sons, New York 1999. [16] J. Mijatovic, WH. Binder, H. Gruber, Characterization of surface modified silica nanoparticles by ${ }^{29} \mathrm{Si}$ solid state NMR spectroscopy, Mikrochim Acta, 133 (2000) 175-181 [17] U. Görl, A. Hunsche, Am. Chem. Soc., Rubber Div. Meeting, Lousville, Kentucky, October 8-11, 1996

[18] A. Hunsche, U. Görl, A. Muller, M. Knaak, Kautsch. Gummi Kunstst., 50, (1997) 881

[19] A. Hunsche, U, Görl, H.G. Koban, Th. Lehmann, Kautsch. Gummi Kunstst., 51, (1998) 525

[20] U. Görl, J. Munzenberg, H.D. Luginsland, A. Muller, Kautsch. Gummi Kunstst., 52, (1999) 588-598

[21] U. Görl, A. Hunsche, A. Muller, H.G. Koban., Rubber Chem. Technol., 70, (1997) 608623 
[22] S. Mihara, "Reactive processing of silica-reinforced tire rubber: new insight into the time and temperature-dependence of silica rubber interaction", PhD. Thesis, 2009, Dept. of Elastomer Technology and Engineering, Univ. of Twente, Enschede, the Netherlands.

[23] A. Blume, Kautsch. Gummi Kunstst., 64, (2011) 38-43

[24] J.T. Byers, Rubber World, 218, (1998) 38-44

[25] A. Blume, 180th Am. Chem. Soc., Rubber Div. Meeting, Cleveland, Ohio, 22 (2011) October 9-13

[26] J.L. Valentin, P. Posadas, A. Marcos-Fernandez, L. Ibarra, A. Rodriguez, Journal of Applied Polymer Science, 99, (2006) 3222-3229

[27] L.A.E.M. Reuvekamp, S.C. Debnath, J.W.Ten Brinke, P.J.Van Swaaij, J.W.M. Noordermeer, Rubber Chem. Technol., 77, (2004) 34-49

[28] S.S. Sarkawai, W.K. Dierkes, J.W.M. Noordermeer, European Polymer Journal, 49, (2013) 3199-3209

[29] K. Nawamawat, J. Sakdapipanich, D. Mekkriengkrai, Y. Tanaka, Kautsch. Gummi Kunstst., (2008) 518-522

[30] A.H. Eng, S. Kawahara, Y. Tanaka, Rubber Chem. Technol., 67 (1994) 159-168

[31] Y. Tanaka, L. Tarachiwin, Rubber Chem. Technol., 82 (2009) 283-314

[32] S. Amnuaypornsri, J. Sakdapipanich, S. Toki, B.S. Hsiao, N. Ichikawa, Y. Tanaka, Rubber Chem. Technol., 81 (2008) 753-766

[33] A. Blume, J. Fähr, C. Morisse, Rubber World, (2012) 24-29

[34] S.C. Debnath, R.N Data, J.W.M. Noordermeer, Rubber Chem. Technol., 76 (2003) 13111328

[35] U. Görl, J. Munzenberg, H.D. Luginsland, A. Muller, Kautsch. Gummi Kunstst., 52 (1999) 588-598

[36] H.-D. Luginsland, Kautsch. Gummi Kunstst., 53 (2000) 10-23

[37] L.A.E.M Reuvekamp, "Reactive mixing of silica and rubber for tires and engine mounts", Thesis: 2003, Dept. Rubber Technol., Univ. of Twente, Enschede, the Netherlands

[38] W. Kaewsakul, K. Sahakaro, W.K. Dierkes, J.W.M. Noordermeer, Rubber Chem. Technol., 85 (2012) 277-294

[39] H. Hayen, M.M. Alvarez-Grima, S.C. Debnath, J.W.M. Noordermeer, U. Karst, Anal. Chem., 76 (2004) 1063-1068

[40] P. Versloot, J.G Haasnoot, J. Reedijk, M.Van Duin, J. Put, Rubber Chem. Technol., 70 (1997) 106-119

[41] O. Klockmann, A, Hasse, German Rubber Conference 2006, Nuremberg, July 3-6 (2006)

[42] O. Klockmann, P. Albert, A. Hasse, K. Korth, Rubber World, (2006) 36-40 
[43] O. Klockmann, A. Blume, A. Hasse, 172nd Am. Chem. Soc., Rubber Div. Meeting, Cleveland, 87, October 16-18 (2007)

[44] O. Klockmann, 174th Am. Chem. Soc., Rubber Div. Meeting, Louisville, Kentucky, October 14-16 (2008)

[45] Momentive Marketing Bulletin, NXT Z 45 Silane Coupling Agent for Silica_Reinforced Tire Tread Compounds

[46] D. Sitnikova, Poster "NXT silane: Improvement of Rolling Resistance and Wet Traction", RUBBERCON 2016, 8-9 June 2016, Tampere, Finland

[47] M. York, Tire Tech 2015, Cologne, February 10-12 (2015)

[48] O. Klockmann, J. Hahn, H. Scherer, International Rubber Conference 2009, Nuremberg, June 29-July 2 (2009)

[49] A.R. Payne, Rubber Plast. Age, 42 (1961) 963-967

[50] A.R. Payne, Rubber Chem. Technol., 39 (1966) 365-374

[51] A.Y. Coran, J.-B. Donnet, Rubber Chem. Technol., 65 (1992) 1016-1041

[52] H.-D. Luginsland, J. Fröhlich, A. Wehmeier, Rubber Chem. Technol., 75 (2002) 563-579

[53] S. Wolff, Rubber Chem. Technol., 69 (1996) 325-346

[54] G. Kraus, Rubber Chem. Technol., 44 (1971) 199-213

[55] A.I. Medalia, Journal of Colloid and Interface Science, 32 (1970) 115-131

[56] P.P.A. Smit, Rubber Chem. Technol., 41 (1968) 1194-1202

[57] I. Pliskin, N. Tokita, J. Appl. Polym. Sci., 16 (1972) 473-492

[58] J. O’Brien, E. Cashell, G.E. Wardell, V.J. McBrierty, Macromolecules, 9 (1976) 653-660

[59] S. Wolff, M.J. Wang, E.H. Tan, Rubber Chem. Technol., 66 (1993) 163-177

[60] L. Guy, S. Daudey, P. Cochet, Y. Bomal, Kautsch. Gummi Kunstst., (2009) 383-391

[61] C.J. Lin, W.L. Hergenrother, E. Alexanian, G.G.A. Böhm, Rubber Chem. Technol., 75 (2002) 865-890

[62] S. Mihara, R.N. Datta, J.W.M. Noordermeer, Rubber Chem. Technol., 82 (2009) 524-540

[63] M. R. Kresja, J. L. Koenig, Rubber Chem. Technol., 66 (1993) 376-410

[64] R.S. Clough, J.L. Koenig, Rubber Chem. Technol., 62 (1989) 908-927

[65] A.M. Zaper, J.L. Koenig, Rubber Chem. Technol., 60 (1987) 252-277

[66] J.R. Wolfe, T.L. Pugh, A.S. Killian, Rubber Chem. Technol., 41 (1968) 1329-1338

[67] S. Horiuchi, H. Dohi, Langmuir, 22 (2006) 4607-4613

[68] N.J. Morrison, M. Porter, Rubber Chem. Technol., 57 (1984) 63-85

[69] L. Bateman, The Chemistry and Physics of Rubber-like Substances, Maclaren, London (1963) chap. 15

[70] A. Y. Coran, Journal of Applied Polymer Science, 87 (2003) 24-30 
[71] L. Bateman, R.W. Glazebrook, C.G. Moore, J. Chem. Soc., (1958) 2848-2856

[72] T.D. Skinner, Rubber Chem. Technol., 45 (1972) 182-192

[73] J.R. Wolfe, Rubber Chem. Technol., 41 (1968), 1339-1347

[74] J. Hahn, M. Runk, M. Schollmeyer, U. Theimer, E.Walter, Kautsch. Gummi Kunstst., 51 (1998) 206-211

[75] P. Versloot, J.G. Haasnoot, J. Reedijk, M. Van Duin, E.F.J. Duynstee, J. Put, Rubber Chem. Technol., 65 (1991) 343-349 


\section{Chapter 3}

\section{Model compound study-1; \\ Reactivity study of mercapto-silane and sulfide-silane with various types of olefin}

\section{3-1 Introduction}

In the silica filled compounds, it is essential to use silane coupling agents in order to increase the achievable silica dispersion level by overcoming the low compatibility between rubber and silica as well as by obtaining a high reinforcing effect. Recently, as a more effective way to improve the silica dispersion level, mercapto-silanes came into focus.[1-6] On one hand, the usage of mercapto-silanes instead of conventional sulfide-silanes can result in a lower Payne effect value and also a lower tan $\delta$ at $60^{\circ} \mathrm{C}$ value. On the other hand, their use can cause a higher Mooney viscosity as well as a shorter scorch time compared to sulfide-silanes, which result in processability difficulties. Therefore, the use of mercaptosilanes is still challenging.

It has been reported that the comparison of mercapto-, disulfide- and tetrasulfide-silanes with having all the same triethoxy groups shows a similar silanization reaction rate.[7] Hence, it seems to be more important to understand deeply the reaction between the sulfur side of the silane and the rubber (silane - polymer reaction) in order to clarify the mechanisms for the improved silica dispersion as well as worse processability in the mercapto-silane systems.

To elucidate the silane - polymer reaction, the model olefin study will be used as a first approach in this chapter. The model olefin study, which uses liquid olefins instead of solid rubbers, has merits in terms of increasing applicable analytical methods resulting in a higher possibility to identify reaction products and pathways. Thus, this technique has already been used a lot to elucidate the vulcanization mechanism. Furthermore, it has also been used for the investigation of the reaction between a sulfide-silane and a rubber. The detection of cross-linked products between two mol of tetra-methyl-ethylene (TME) via 
sulfur chains with tracing the sulfur rank distribution of TESPT during heating with TME delivered the confirmation of the sulfur donor effect of TESPT.[8,9] Furthermore, in the reaction mixture of trans-3-hexene $(\mathrm{T} 3 \mathrm{H})$ and TESPT, the generation of the adducts formed thermal cleavage of TESPT and subsequent addition of the formed $(\mathrm{EtO})_{3} \mathrm{Si}_{(}\left(\mathrm{CH}_{2}\right)_{3} \mathrm{~S}_{\mathrm{x}}(\mathrm{x}=2-5)$ to a T3H molecule was proposed by the obtained mass value in the LC/MS measurement.[10]

(a)

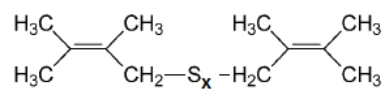

(b)

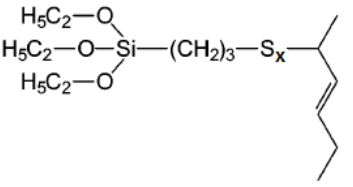

Figure 3-1: Proposed reaction product between (a) TME and TESPT, (b) T3H and TESPT

The representative rubbers used for tire tread compounds are SBR, BR, IR and NR, which have a different molecular structure. Moreover, there are various micro structures inside SBR or BR rubber. These differences lead to different Tg and crystallinity as well as different physical properties in rubbers. In addition, those affect the chemical reaction aspects of the rubbers such as a cross-linking structure and the oxidation process accompanying the degradation of the rubber. Considering the above mentioned points, it can be expected that the structure of polymer affects their reactivity with silanes, therefore, various different structures of olefin shown in Figure 3-2 will be investigated. 3-methyl-1-pentene (3m1p) and 1,7-Octadiene (Ocd) have one or two terminal double bonds in the molecule, especially $3 \mathrm{~m} 1 \mathrm{p}$ can be considered as a model substance for the vinyl structure in BR or SBR. Cis-3hexene $(\mathrm{C} 3 \mathrm{H})$ and $\mathrm{T} 3 \mathrm{H}$ are chosen as model substances for the cis or trans double bond in $\mathrm{BR}$ or SBR, trans-3-methyl-3-hexene (T3M3H) as model for the double bond in the isoprene type of rubber such as NR or IR. TME which has four equivalent methyl group is selected to compare with the above mentioned five olefins and also with the results already described in the literature.

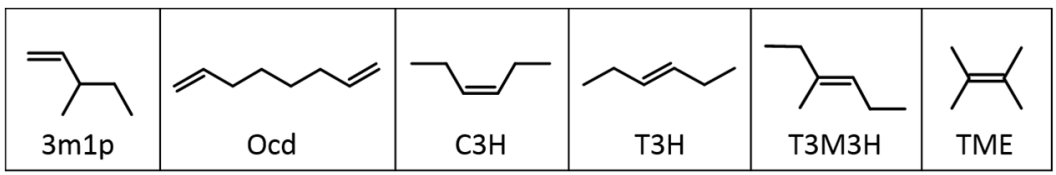

Figure 3-2: The structure of model olefins used in this chapter 


\section{3-2 Experimental}

\section{3-2-1 Sample preparation}

The used materials including their molecular weight of this study are summarized in Table 3-1.

Table 3-1; Material list for the model olefin study

\begin{tabular}{cccc}
\hline Material & Abbreviations & Source & $\begin{array}{c}\text { Molecular weight } \\
(\mathrm{g} / \mathrm{mol})\end{array}$ \\
\hline 3-methyl-1-pentene & 3m1p & Sigma-Aldrich & 84.16 \\
1,7-octadiene & Ocd & Sigma-Aldrich & 110.20 \\
cis-3-hexene & C3H & Sigma-Aldrich & 84.16 \\
trans-3-Hexene & T3H & Sigma-Aldrich & 84.16 \\
trans-3-methyl-3-Hexene & T3M3H & Sigma-Aldrich & 98.19 \\
2,3-Dimethyl-2-butene & TME & Sigma-Aldrich & 84.16 \\
\hline (3-Mercaptopropyl)triethoxysilane & Si 263 & Evonik & 238.42 \\
bis(triethoxysilylpropyl)-tetrasulfide & Si 69 & Evonik & 532.00 \\
\hline Decane & \multicolumn{3}{c}{ Sigma-Aldrich } \\
\hline
\end{tabular}

Before the preparation of the samples, the silane and decane were both flushed with nitrogen. Olefins were used without this pre-preparation considering the relatively lower boiling point. An isomolar mixture of only a silane and an olefin was weighed for the identification of reaction products. Twice as much mole of decane than olefin and silane was added for the quantitative measurements as an internal standard. The mixture was divided into different small glass tubes $(2.0 \mathrm{ml})$. The reaction was carried out by placing the tubes in a device named PL-SP 260VS which can shake and heat. Each tube was heated up to the desired specific temperature for pre-determined durations. After the reaction, the tubes were cooled immediately in an ice bath to stop the reaction. Samples were prepared for the further chemical analysis.

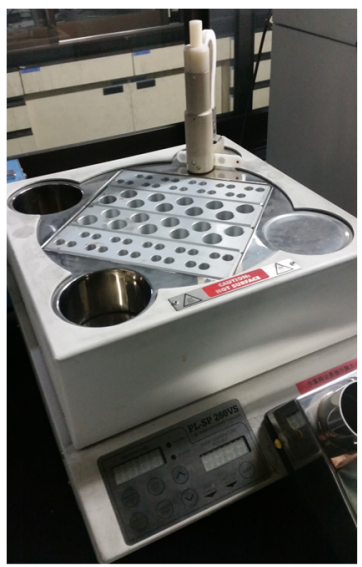

Figure 3-3 Heating machine (PL-SP 260VS) 


\section{3-2-2 Analytical methods}

\section{3-2-2-1 GC Analysis}

The Gas Chromatography (GC) measurement was carried out using GC-2010 Plus ATF (SHIMADZU). The used column type was $95 \%$ dimethyl- and $5 \%$ diphenylpolysiloxane (phenomenex, ZB-5(phase)) with the length of $30 \mathrm{~m}$. The injection volume was $1.0 \mu \mathrm{l}$ with a split ratio 200 . The setting temperature program was as following; injection part $=250^{\circ} \mathrm{C}$, column program $=50^{\circ} \mathrm{C}$ at 3 minutes $\rightarrow$ increases $10^{\circ} \mathrm{C}$ per minute up to $320^{\circ} \mathrm{C}$, detector $=320^{\circ} \mathrm{C}$. As a detector, a Flame Ionization Detector (FID) was used.

\section{3-2-2-2 LC/MS Analysis}

The Liquid Chromatography - Mass spectrometry (LC/MS) analysis was performed using an Alliance 2695 / ZQ2000 (Waters). A reverse phase column (HydrosphereC18 (YMC)) with a length of $100 \mathrm{~mm}$ and an internal diameter of $2.0 \mathrm{~mm}$ was used for the separation. The mobile phase was a mixture of methanol and water with a gradient mixture ratio starting from $65 / 35$ to $100 / 0$ in 15 minutes and $97 / 3$ in 30 minutes. The flow rate of the mobile phase of $0.2 \mathrm{ml} / \mathrm{min}$ was optimized for separation and MS ionization. The injected volume inside the HPLC system was $5 \mu \mathrm{l}$. After passing the column, the different ingredients were separated and ionized using the Electrospray Ionization (ESI) technique. Protonation in positive ion mode leads to adducts with sodium or potassium or ammonium. The created ions were identified according to their $\mathrm{m} / \mathrm{z}$ ratio.

\section{3-2-2-3 GC/MS Analysis}

The Gas Chromatography - Mass spectrometry (GC/MS) analysis was conducted using a 7890B GC System (Agilent Technologies) in combination wtih a JMS-Q1050GC (JEOL DATUM). The used column type was $95 \%$ dimethyl- and $5 \%$ diphenylpolysiloxane (Ultra ALLOY-5 Capillary Column) with the length of $30 \mathrm{~m}$. The injection volume was $1.0 \mu \mathrm{l}$ with a split ratio 100 . The setting temperature program was as following; injection part $=300^{\circ} \mathrm{C}$, column program $=50^{\circ} \mathrm{C}$ at 3 minutes $\rightarrow$ increases $15^{\circ} \mathrm{C}$ per minute up to $320^{\circ} \mathrm{C}$.

\section{3-2-2-4 NMR Analysis}

The samples were dissolved in deuterated chloroform $\left(\mathrm{CDCl}_{3}\right)$ containing tetramethylsilane as an internal standard and characterized by Nuclear Magnetic Resonance (NMR) spectroscopy (AV 400M, Bruker Biospin). ${ }^{1} \mathrm{H}$ NMR and ${ }^{13} \mathrm{C}$ NMR spectra were recorded at $400 \mathrm{MHz}$ and $100 \mathrm{MHz}$, respectively. A DEPT (Distortionless Enhancement 
by Polarization Transfer) measurement was used to distinguish different carbon atom substitutions. COSY (Homonuclear Correlated Spectroscopy) was applied to identify the position and the surrounding of the protons. HSQC (Heteronuclear Single Quantum Coherence) was used to identify the surrounding of the carbons. Finally, HMBC (Heteronuclear Multi-Bond Connectivity) was utilized to clarify the exact structure of the product by evaluating the long-range heteronuclear coupling.[11]

\section{3-2-2-5 Chemical shift calculations}

The ${ }^{1} \mathrm{H}$ and ${ }^{13} \mathrm{C}$ chemical shift were calculated by using the commercially available software program $\mathrm{ACD} / \mathrm{C}+\mathrm{H}$ NMR (Version 12.0).

\section{3-3 Results and Discussion}

\section{3-3-1 Comparison of two silane systems in combination with $3 \mathrm{~m} 1 \mathrm{p}$ or $\mathrm{C3H}$}

The reaction of two silanes, Si 263 and Si 69, with 3m1p and $\mathrm{C} 3 \mathrm{H}$ respectively are compared. Figure 3-4 shows the $3 \mathrm{~m} 1 \mathrm{p}$ peak area ratio relative to the original value in the GC measurement in the Si 263 and Si 69 systems as a function of heating time at different temperatures. Although the decrease of the amount of $3 \mathrm{~m} 1 \mathrm{p}$ in both systems with increasing heating time is observed, the reaction rate inside the Si 263 system is higher for all temperatures.
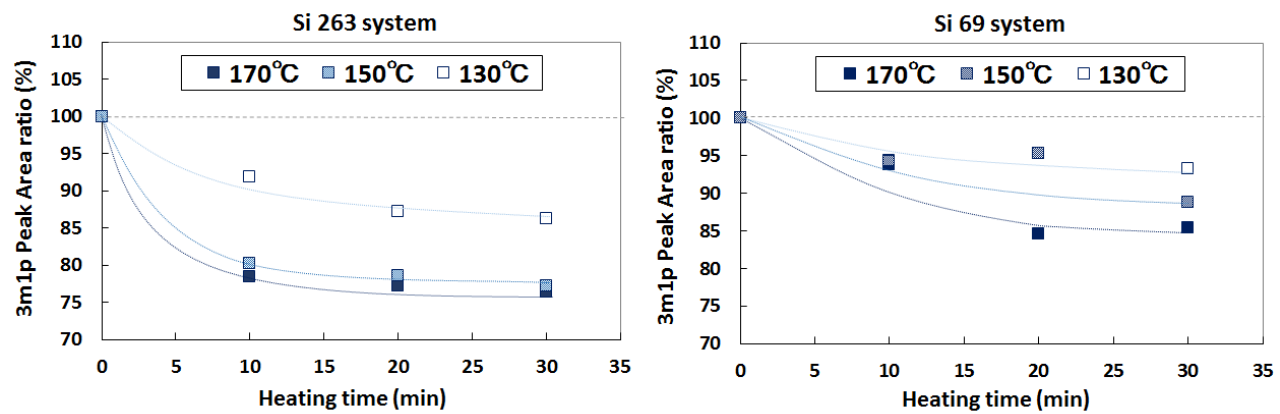

Figure 3-4: Change of the $3 \mathrm{~m} 1 \mathrm{p}$ concentration with heating time (in the Si 263 system = left, in the Si 69 system = right) 
Comparable figures are drawn for the $\mathrm{C} 3 \mathrm{H}$ systems but this time using the data from the ${ }^{13} \mathrm{C}$ NMR measurements to detect the amount of remaining $\mathrm{C} 3 \mathrm{H}$, as shown in Figure 3-5. Here, again, a big difference regarding the reaction rate of $\mathrm{C} 3 \mathrm{H}$ between Si 263 and Si 69 systems can be observed. The concentration of $\mathrm{C} 3 \mathrm{H}$ in the $\mathrm{Si} 263$ system drastically decreases especially at 150 and $170^{\circ} \mathrm{C}$ after a short heating time. The amount is reduced by 50 to $70 \%$ just after 5 minutes of heating at 150 and $170{ }^{\circ} \mathrm{C}$, respectively. When the reaction rates of both olefins are compared in the same Si 263 system, that of $\mathrm{C} 3 \mathrm{H}$ is significantly higher than that of $3 \mathrm{~m} 1 \mathrm{p}$. Therefore, it seems that $\mathrm{Si} 263$ has a higher reactivity to $\mathrm{C} 3 \mathrm{H}$ than to $3 \mathrm{~m} 1 \mathrm{p}$. However, when the concentration change of Si 263 in both olefin systems is compared as shown in Figure 3-6, the results show a different tendency. Despite the significant decrease of the $\mathrm{C} 3 \mathrm{H}$ content during heating, the concentration of $\mathrm{Si} 263$ in the $\mathrm{C} 3 \mathrm{H}$ system decreases surprisingly very slowly with increasing heating time. The $3 \mathrm{~m} 1 \mathrm{p}$ system shows a higher conversion of Si 263 than that in the $\mathrm{C} 3 \mathrm{H}$ system, and the extent and the speed of decrease of Si 263 look similar as that of $3 \mathrm{~m} 1 \mathrm{p}$.
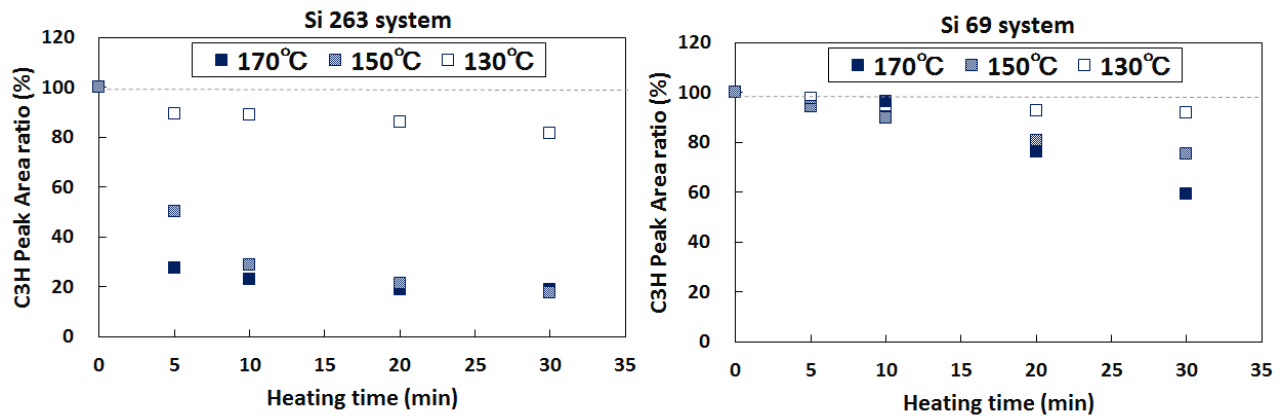

Figure 3-5: Change of the $\mathrm{C} 3 \mathrm{H}$ concentration with heating time (in the Si 263 system = left, in the Si 69 system = right)

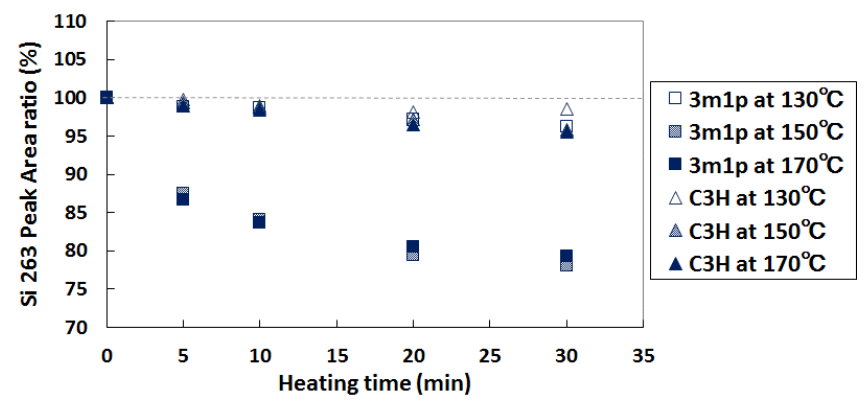

Figure 3-6: Change of the Si 263 concentration with heating time 
These results so far imply that the presence of Si 263 has a stronger influence on the olefin than Si 69, and the reaction mechanism varies depending on the type of olefin. These difference seems to affect also the physical properties and processability in silica filled compounds. Therefore, to elucidate possible reactions in each combination is crucial in order to control these effects and also to establish effective application ways for these silanes.

As a next step the reaction mechanisms and the structure of these reaction products were identified with different analytical techniques such as Gas Chromatography/Mass Spectrometry (GC/MS), Liquid chromatography-Mass Spectrometry (LC/MS), ${ }^{1} \mathrm{H}$ and ${ }^{13} \mathrm{C}$ NMR and three two-dimensional NMR (2D-NMR) spectroscopies.

\section{3-3-2 Si 263 system}

In order to evaluate the reaction mechanism including the influence of the double bond structure and the exact structure of the reaction products six different following model olefins were used. $3 \mathrm{~m} 1 \mathrm{p}$ and Ocd have one or two terminal double bonds in the molecule, $3 \mathrm{~m} 1 \mathrm{p}$ has a vinyl group as the model for BR or SBR which contains vinyl group as well. $\mathrm{C} 3 \mathrm{H}$ and $\mathrm{T} 3 \mathrm{H}$ were chosen as model substances for the cis or trans double bond in BR or SBR, $\mathrm{T} 3 \mathrm{M} 3 \mathrm{H}$ as model for the double bond in the isoprene type of rubber such as NR or IR. TME was selected for the comparison with the above mentioned five olefins and also to compare the results from this model olefin with the results already described in the literature.

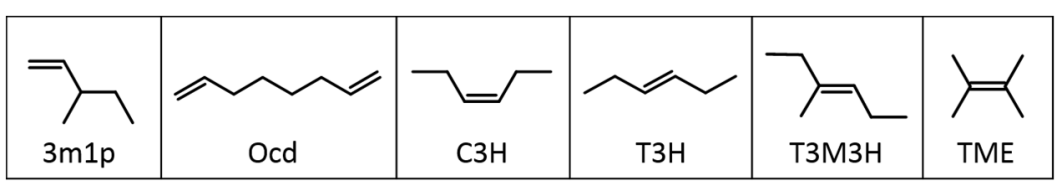

\section{3-3-2-1 3m1p / Si 263 system}

3-methyl-1-pentene (3m1p)

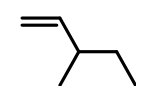

Figure 3-7 shows the GC spectra of the $3 \mathrm{~m} 1 \mathrm{p} / \mathrm{Si} 263$ system before and after heating at $170^{\circ} \mathrm{C}$ for 30 minutes. By heating, a new peak arises at 17 minutes of a retention time. The mass chromatogram of this peak is shown in Figure 3-8. The maximum and 2 nd maximum mass values are 322 and $277 \mathrm{~m} / \mathrm{z}$ respectively. The mass of 322 corresponds to the sum of the molecular weight between one molecule of Si 263 and one of $3 \mathrm{~m} 1 \mathrm{p}$. The mass peak 277 corresponds to a "322" molecule which has lost one ethoxy group. Furthermore, the fragments in this chromatogram contain same fragmentation pattern of both Si 263 and 
$3 \mathrm{~m} 1 \mathrm{p}$ elements. Therefore, a one to one reaction product between $3 \mathrm{~m} 1 \mathrm{p}$ and Si 263 seems to be the resulting structure. Furthermore, an additional small increase of the concentration of di-sulfide silane is also confirmed by checking the Mass chromatogram of the peak at 23 minutes, as shown in Figure 3-9.
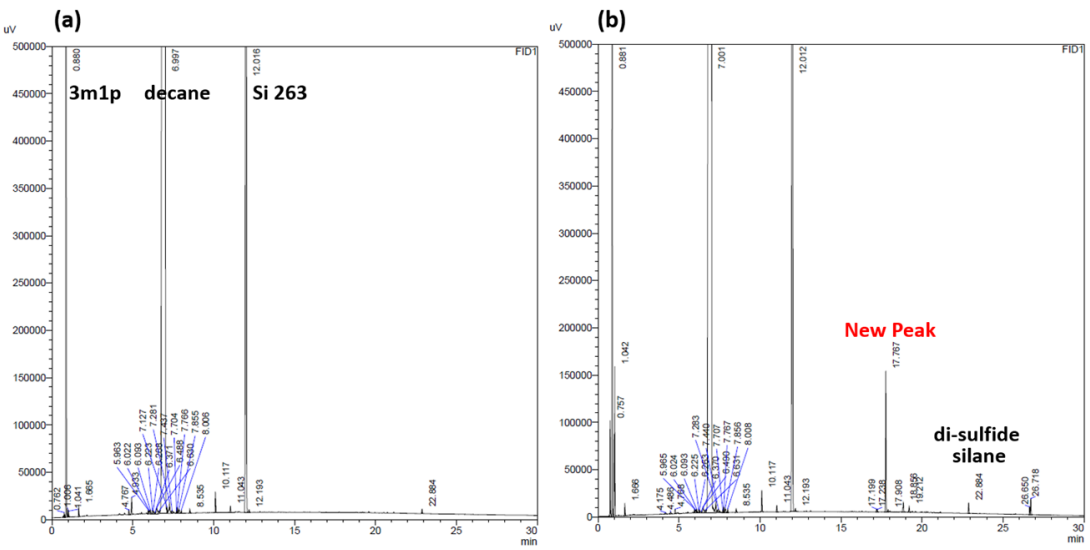

Figure 3-7: GC-spectra of 3m1p / Si 263 system (a) before heating and (b) after heating at $150{ }^{\circ} \mathrm{C}$ for 30 minutes

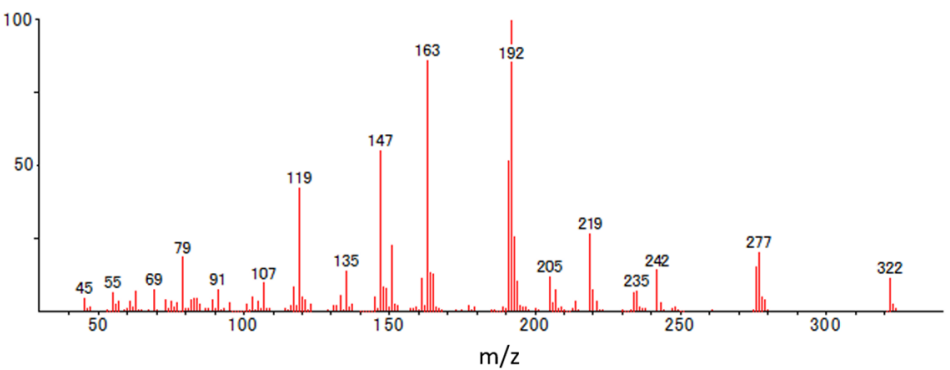

Figure 3-8: Mass chromatogram of the new resulting peak at 17 minutes inside the heated $3 m 1 p$ and Si 263 system (GC/MS measurement)

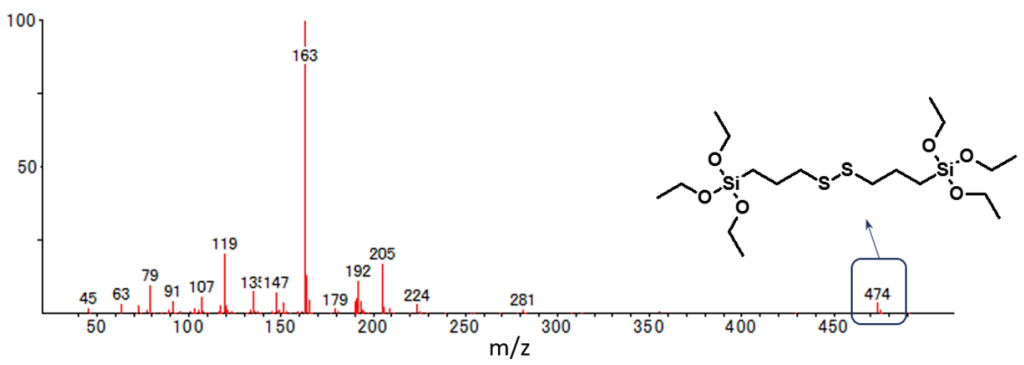

Figure 3-9: Mass chromatogram of the peak at retention time at 22.8 minutes inside the heated 3m1p and Si 263 system (GC/MS measurement) 
Figure 3-10 shows the ${ }^{1} \mathrm{H}$ NMR spectra of the $3 \mathrm{~m} 1 \mathrm{p} / \mathrm{Si} 263$ system before and after heating at $170^{\circ} \mathrm{C}$ for 30 minutes. As characteristic points, the peak shape change is observed at the chemical shift range of $2.5 \mathrm{ppm}$, which corresponds to the proton atom at the carbon atom next to the sulfur atom, therefore, the environmental change around this proton atom can be indicated, which means that the mercapto group may have reacted with a carbon atom in $3 \mathrm{~m} 1 \mathrm{p}$. Based on the information so far, the identification of further new peaks in the ${ }^{13} \mathrm{C}$ NMR spectra was started using various NMR techniques and a prediction software.

In the ${ }^{13} \mathrm{C}$ NMR spectrum (Figure 3-11), ten new peaks which have a similar peak intensity are observed in the range of 0 to $60 \mathrm{ppm}$ which is considered as the area where single bond carbon atoms can be detected. Seven of them can be judged to have a $\mathrm{CH}_{2}$ structure by the support of the ${ }^{13} \mathrm{C}$ DEPT135 spectrum. Although three structures can be considered as a possible one to one reaction product as shown in Figure 3-12, only structure 1 fits to a structure which has the required seven $\mathrm{CH}_{2}$ carbon atoms. The chemical shifts of this structure 1 were calculated by the prediction software, and then, the identification of these new peaks was possible by combining the predicted data with the obtained information from the ${ }^{13} \mathrm{C}$ DEPT measurement (Table 3-2).

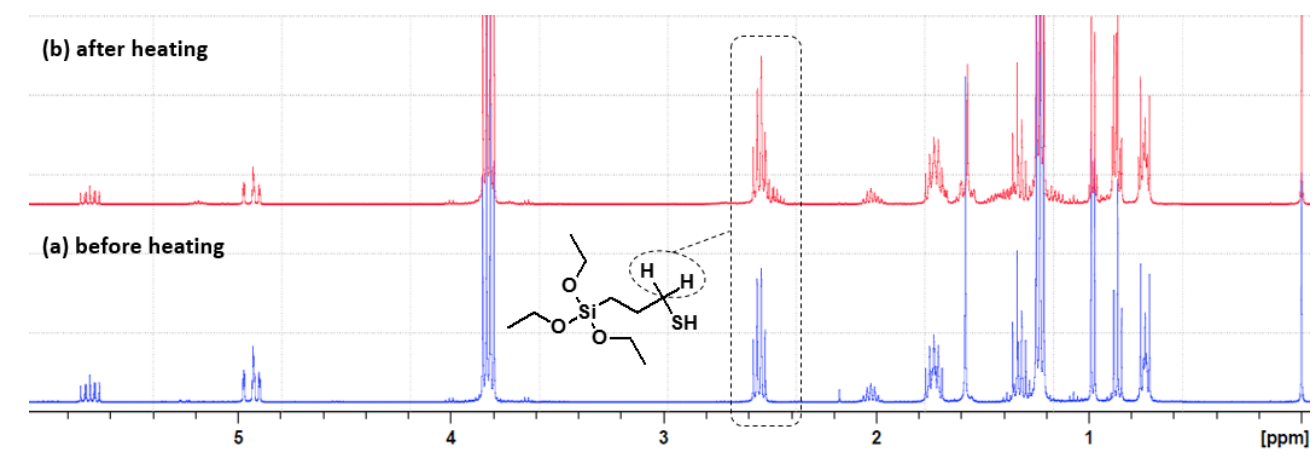

Figure 3-10: ${ }^{1} \mathrm{H}$ NMR spectra of the $3 \mathrm{~m} 1 \mathrm{p} / \mathrm{Si} 263$ mixture (a) before and (b) after heating for 30 minutes at $170^{\circ} \mathrm{C}$ 
(c) DEPT135

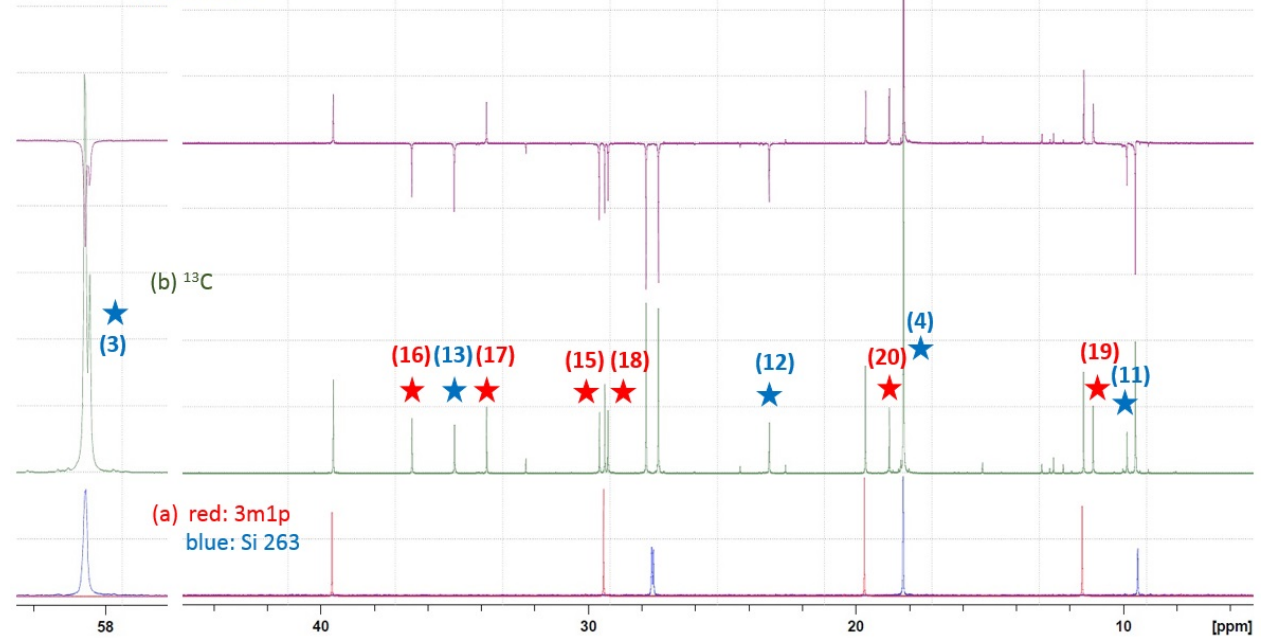

Figure 3-11: NMR spectra; (a) ${ }^{13} \mathrm{C}$ of raw materials, (b) ${ }^{13} \mathrm{C}$ and $(\mathrm{c}){ }^{13} \mathrm{C}$ DEPT135 of the $3 \mathrm{~m} 1 \mathrm{p}$ / Si 263 mixture after heating for 30 minutes at $170^{\circ} \mathrm{C}$

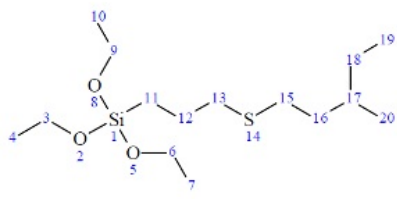

structure 1

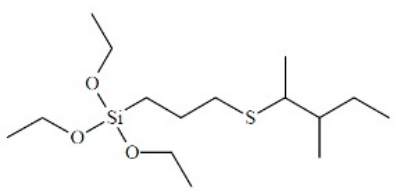

structure 2

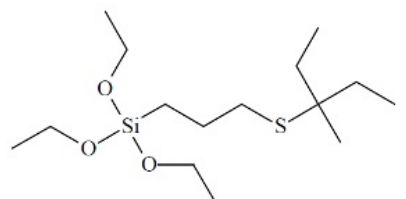

structure 3

Figure 3-12: Possible one to one reaction products between 3m1p and Si 263

Table 3-2: Identification of the reaction product

\begin{tabular}{|c|c|c|c|c|}
\hline $\begin{array}{c}\text { Assign- } \\
\text { ment }\end{array}$ & $\begin{array}{c}\text { Chemical } \\
\text { shift } \\
\text { (ppm) } \\
{ }^{13} \mathrm{C}\end{array}$ & $\begin{array}{c}{ }^{13} \mathrm{C} \\
\text { DEPT135 }\end{array}$ & $\begin{array}{l}\text { Chemical } \\
\text { shift } \\
\text { calculated } \\
\text { (ppm) } \\
{ }^{13} \mathrm{C}\end{array}$ & $\begin{array}{l}\text { Chemical shift } \\
\text { calculated } \\
\text { (ppm) } \\
{ }^{1} \mathrm{H}\end{array}$ \\
\hline (11) & 9.88 & $\mathrm{CH}_{2}$ & 10.09 & $0.60-0.66$ \\
\hline (19) & 11.13 & $\mathrm{CH}_{3}$ or $\mathrm{CH}$ & 11.30 & $0.86,0.88,0.89$ \\
\hline (4) & 18.22 & $\mathrm{CH}_{3}$ or $\mathrm{CH}$ & 18.19 & $1.17,1.19,1.21$ \\
\hline (20) & 18.76 & $\mathrm{CH}_{3}$ or $\mathrm{CH}$ & 19.29 & $0.86,0.87$ \\
\hline (12) & 23.25 & $\mathrm{CH}_{2}$ & 24.63 & $1.59-1.67$ \\
\hline (18) & 29.28 & $\mathrm{CH}_{2}$ & 29.50 & $\begin{array}{l}1.09-1.16 \\
1.28-1.37\end{array}$ \\
\hline (15) & 29.61 & $\mathrm{CH}_{2}$ & 30.73 & $2.40,2.42,2.44$ \\
\hline (17) & 33.82 & $\mathrm{CH}_{3}$ or $\mathrm{CH}$ & 34.07 & $1.21,1.27-1.33$ \\
\hline (13) & 35.03 & $\mathrm{CH}_{2}$ & 34.17 & $2.38-2.41$ \\
\hline (16) & 36.61 & $\mathrm{CH}_{2}$ & 35.92 & $\begin{array}{l}1.39-1.45 \\
1.56-1.64\end{array}$ \\
\hline (3) & 58.16 & $\mathrm{CH}_{2}$ & 58.73 & 3.74-3.79 \\
\hline
\end{tabular}



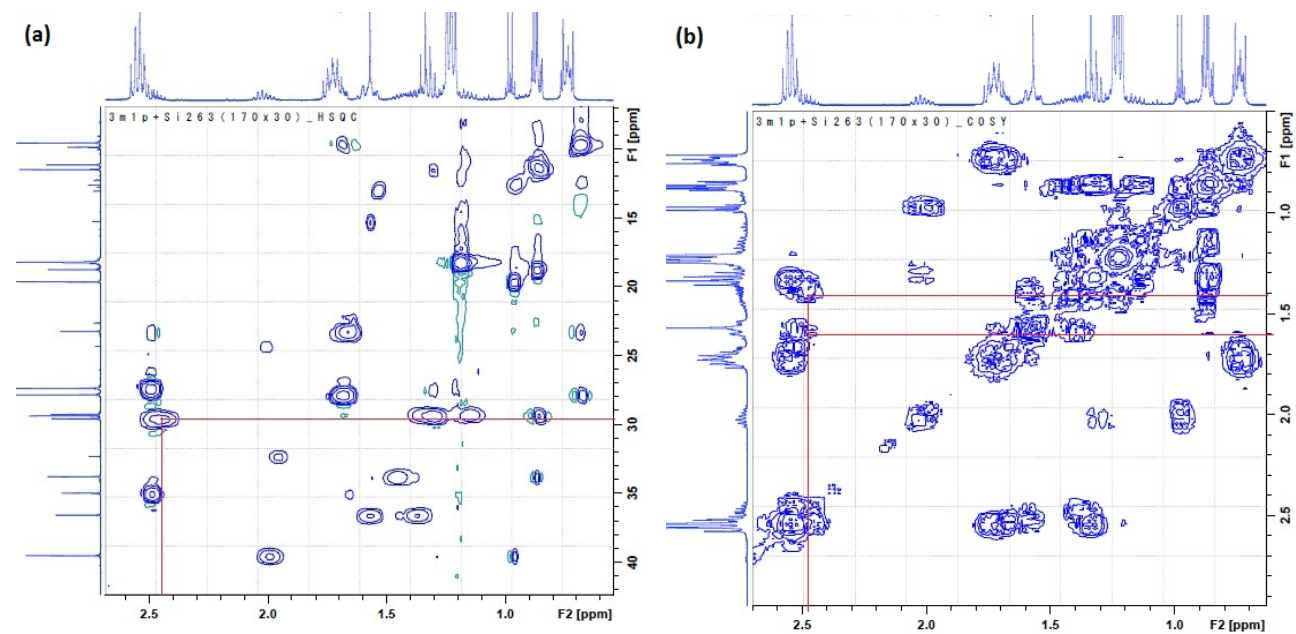

Figure 3-13: 2D-NMR spectra of the 3m1p / Si 263 mixture after heating for 30 minutes at $170^{\circ} \mathrm{C}$; (a)HSQC, (b)COSY

The characteristic chemical shifts of the product are the carbon atom (No. 15 in Figure 312) from $3 \mathrm{~m} 1 \mathrm{p}$ which is next to the sulfur atom and also the hydrogen atoms at the carbon atom, which are expected to appear at 30.7 and $2.4 \mathrm{ppm}$, respectively. It is confirmed that there is a correlation between these chemical shifts by the HSQC measurement. Furthermore, the chemical shift of the hydrogen atom at the carbon atom No. 16 is predicted to be at 1.4 and $1.6 \mathrm{ppm}$, and due to the fact that a correlation between this hydrogen atom and the hydrogen atom at the carbon atom No.15 is observed in the COSY measurement, it can be confirmed that the carbon atoms No. 15 and No. 16 are situated next to each other. These information confirms the generation of the structure 1 in Figure 3-12 which means that Si 263 was added at the end of the double bond of $3 \mathrm{~m} 1 \mathrm{p}$. This structure is an Anti-Markovnikov type structure. Considering the intermediate stability point of view, the generation of an Anti-Markovnikov type structure leads to the conclusion that this addition reaction follows a radical pathway. Moreover, the additional generation of di-sulfide silanes were observed which seems to be generated by the dimerization of two mercapto-silane radicals. This is another hint for the existence of mercapto-silane radicals (Figure 3-14).

Actually, the addition reaction of a thiol radical to the double bond is well known as a thiol-ene reaction, and it is also reported that the reaction product shows an Anti- 
Markovnikov type of structure.[12-17] The thiol-ene reaction is widely used for photo polymerization, photo crosslinking reactions and functionalization of polymers.[13] For those purposes, normally photoinitiator, peroxide or azo compounds are used as a radical initiator.[13,15] Once formed, the radicals react with thiol moieties under a hydrogen transfer leading to the formation of thiyl radicals. However, in this model study, mercaptosilane radicals have been created without using any radical initiators. One of the possible reasons of it is a dissociation of the -SH group just by heating. Another possible mechanism is a molecular assisted hemolysis via a formation of an electron-donor/-acceptor (EDA) complex, as shown in Figure 3-15. The generation of a mercapto radical in the presence of an olefin was reported in the literature by the displayed mechanism.[18] The formed mercapto-silane radical reacts at the end of the vinyl double bond. Once the reaction has started, the created intermediate (thioether and carbon centered) radical abstracts a hydrogen radical from a different mercapto-silane molecule and/or a different $3 \mathrm{~m} 1 \mathrm{p}$ molecule. This reaction process seems to proceed very fast. However, this addition reaction process is also known as a reversible reaction.[14] This may be the reason that the generation of the addition product shows a saturation with long heating time (Figure 3-4).

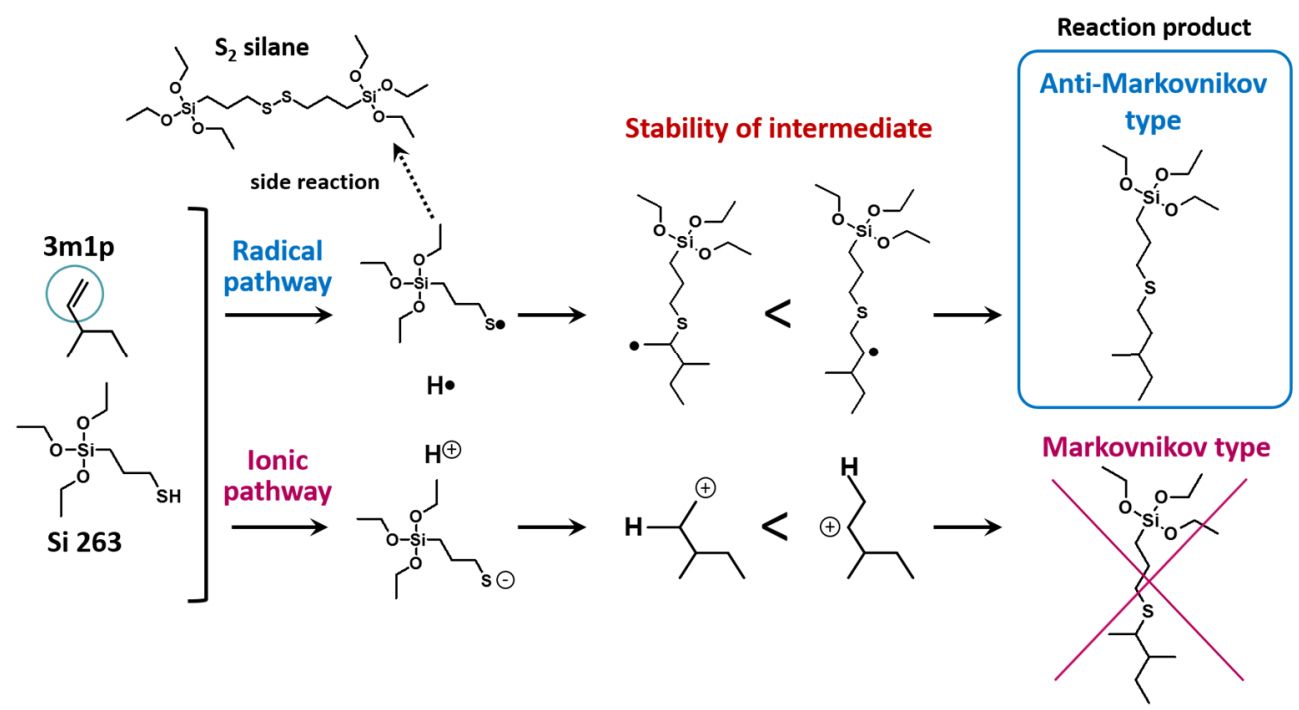

Figure 3-14: Possible mechanism of the reaction between 3m1p with Si 263 


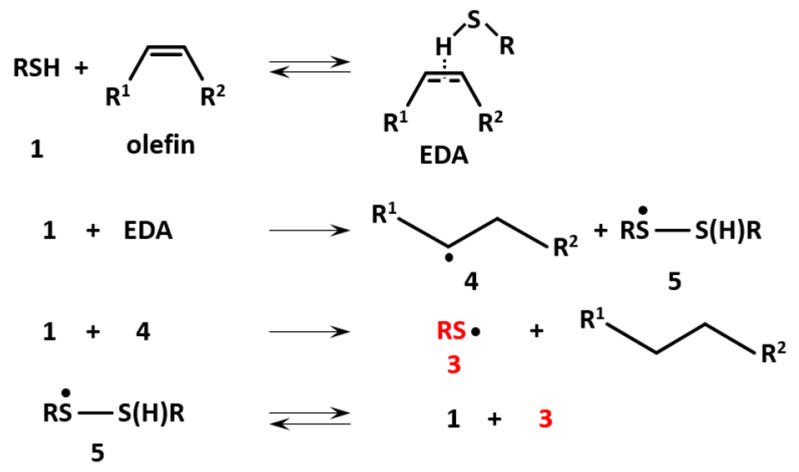

Figure 3-15: Radical formation via an EDA complex of a thiol and an alkene [18]

\section{3-3-2-2 Ocd / Si 263 system}

1,7-octadiene (Ocd)

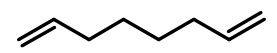

The identification of the reaction product was also carried out in the Ocd system which has a terminal double bond, similar to that one in $3 \mathrm{~m} 1 \mathrm{p}$.

In the ${ }^{1} \mathrm{H}$ NMR spectrum after heating, a significant change of the peak shape is visible in the $2.5 \mathrm{ppm}$ range, which corresponds to the proton atom at the carbon atom located in next to the sulfur atom (Figure 3-16). Moreover, an ESI+ total ion chromatogram in the LC/MS measurement shows two new resulting peaks after heating with a retention times of 18.1 and 21.3 minutes. These peaks are supposed to be the products which are the sodium ion adducts of the reaction products between one Si 263 molecule and one terminal double bond in Ocd (M110 + M238 + 23) and also between two Si 263 molecules and both end of double bonds in Ocd (M110 + $2 \times M 238+23)$ from the information of mass values, respectively, as shown in Figure 3-17.

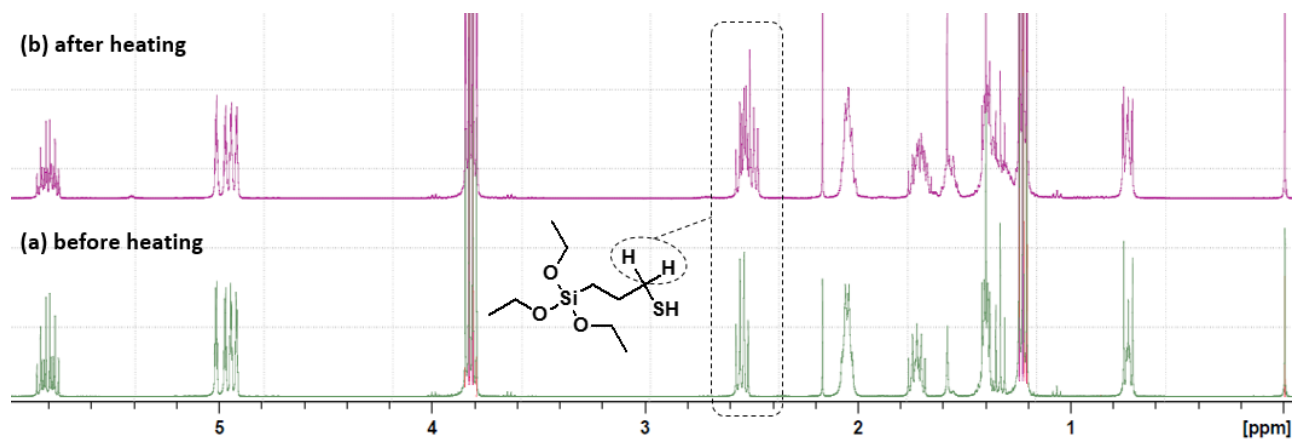

Figure 3-16: ${ }^{1} \mathrm{H}$ NMR spectra of the Ocd / Si 263 mixture (a) before and (b) after heating for 30 minutes at $170^{\circ} \mathrm{C}$ 
The detailed evaluation for the identification of ${ }^{13} \mathrm{C}$ NMR peaks based on the above mentioned information together with various NMR results and the usage of the prediction software successfully reveals the generation of the Anti-Markovnikov type of addition reaction product between Si 263 and Ocd (See Figure 3-18 and Table 3-3).

This result confirms again that Si 263 can react with a terminal double bond in an olefin following a radical pathway.

(a)

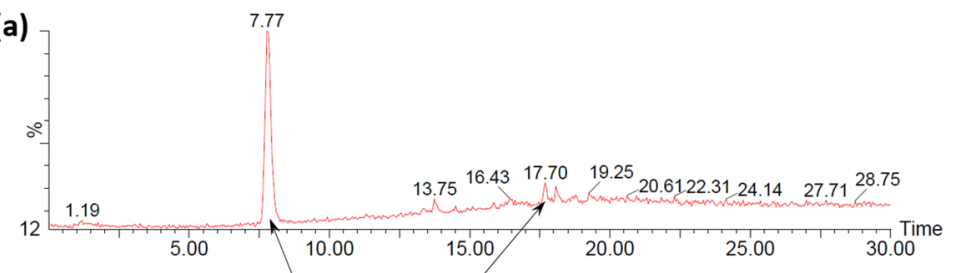

(b)

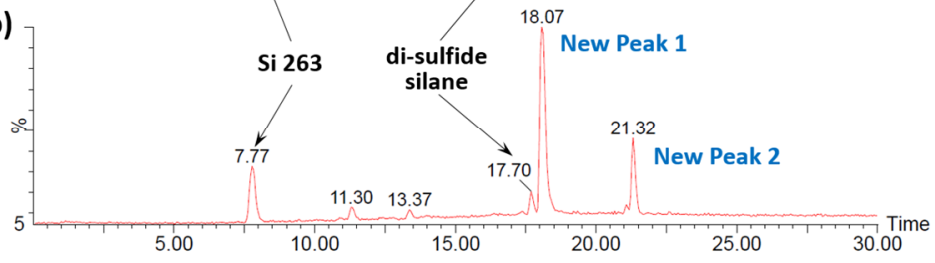

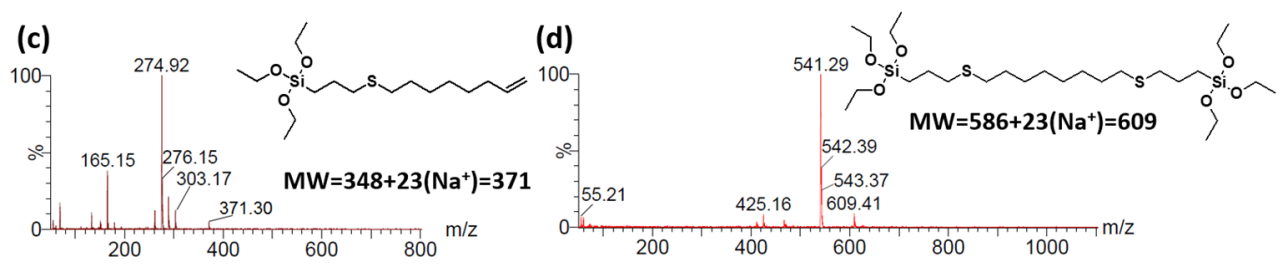

Figure 3-17: LC/MS chromatogram of the Ocd / Si 263 mixture (a) before and (b) after heating for 30 minutes at $170^{\circ} \mathrm{C}$, and Mass chromatogram of the peak at retention time (c) 18.1 minutes and (d) 21.3 minutes in the chromatogram (b) 


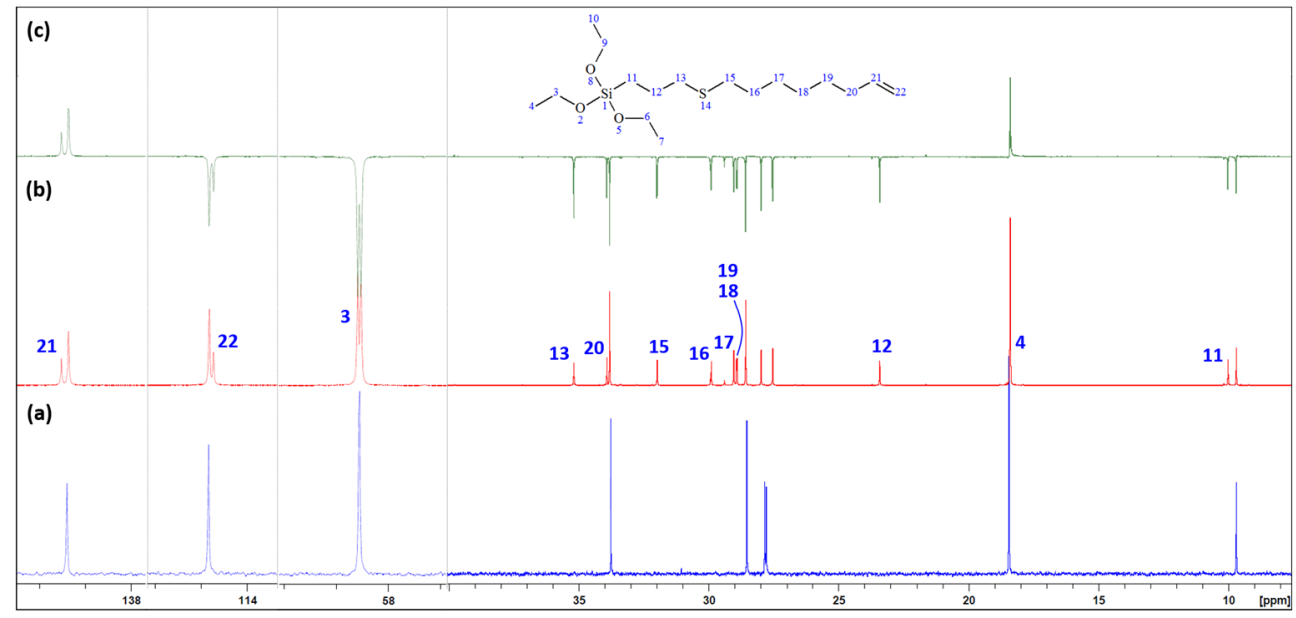

Figure 3-18: NMR spectra of the Ocd / Si 263 mixture; (a) ${ }^{13} \mathrm{C}$ before heating, (b) ${ }^{13} \mathrm{C}$ and (c) ${ }^{13} \mathrm{C}$ DEPT135 after heating for 30 minutes at $170{ }^{\circ} \mathrm{C}$

Table 3-3: Identification of the reaction product

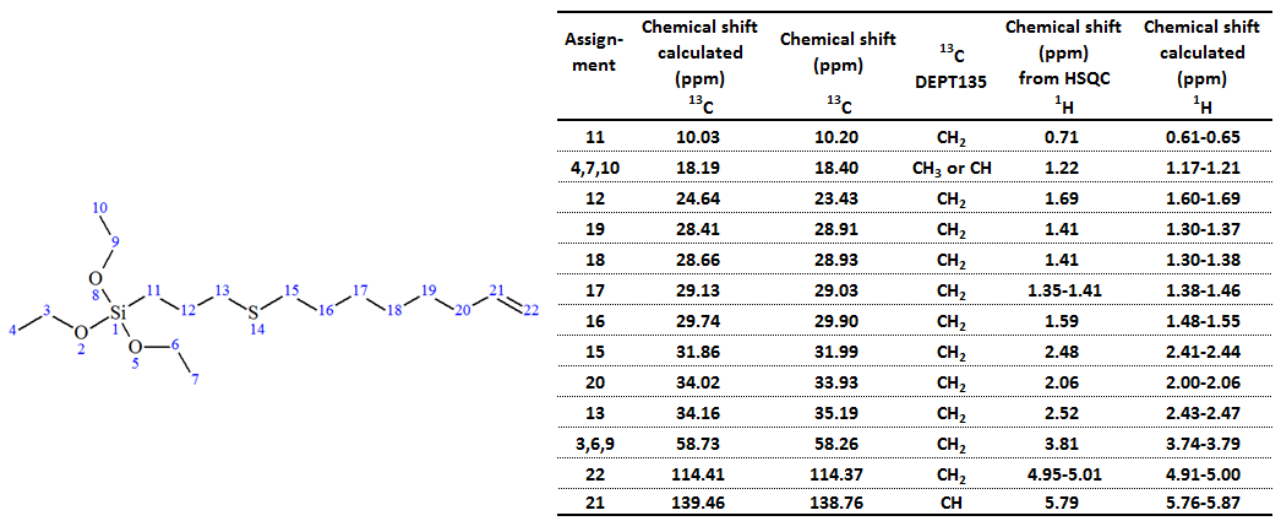




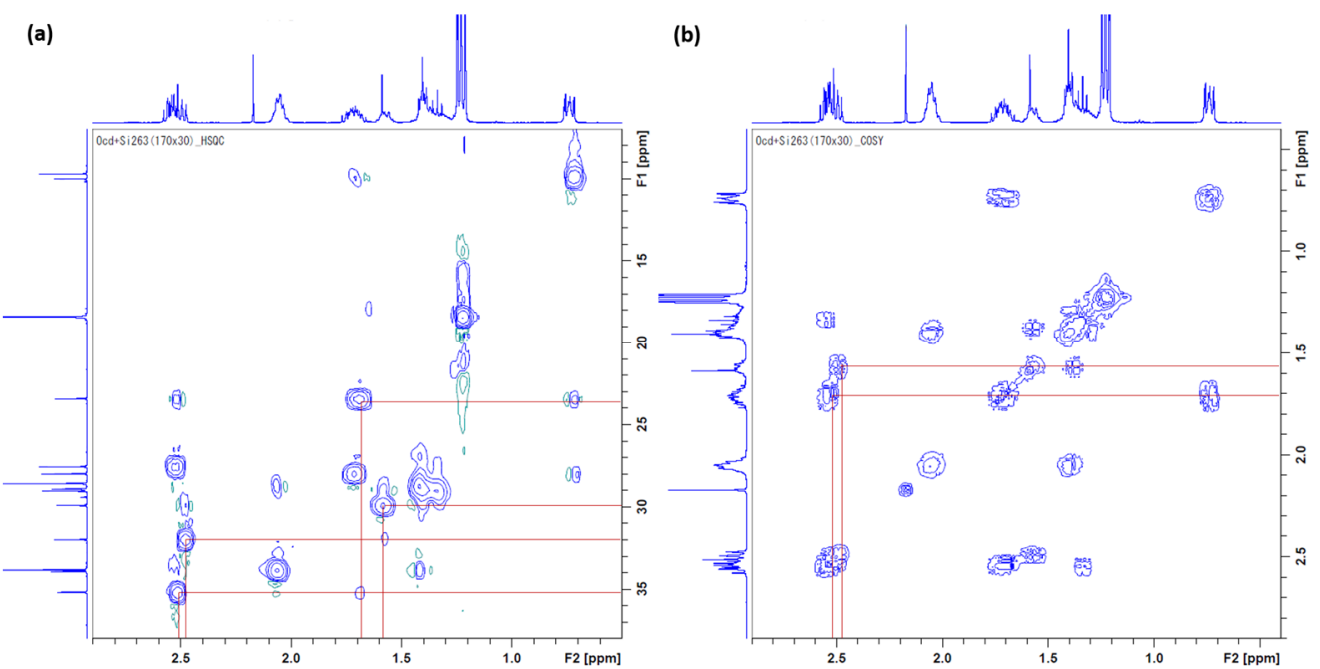

Figure 3-19: 2D-NMR of the Ocd / Si 263 mixture after heating for 30 minutes at $170^{\circ} \mathrm{C}$;

(a)HSQC, (b)COSY

\section{3-3-2-2 C3H / Si 263 system}

cis-3-hexene (C3H)

In this and the next paragraph the model olefins with internal double bonds will be evaluated and compared to those with terminal double bonds. $\mathrm{C} 3 \mathrm{H}$ will be investigated first, T3H in the following chapter 3-3-2-3. It is described in the literature that a cis structure can lead more easily to an addition reaction compared to a trans structure. The reason for this is that a trans structure is thermodynamically more stable and has also a higher steric hindrance effect than a cis structure.[19] In order to know to which degree this point affects the isomerization behavior in the mercapto-silane system, investigations using both cis and trans structures were carried out.

Figure 3-20 shows the ${ }^{1} \mathrm{H}$ NMR spectra before and after heating the $\mathrm{C} 3 \mathrm{H} / \mathrm{Si} 263$ system. Although a peak shape change around $2.5 \mathrm{ppm}$ can be noticed, the degree is small. Considerable changes are rather observed in the chemical shift range of the double bond and the allylic hydrogen, which imply the isomerization of $\mathrm{C} 3 \mathrm{H}$ to trans-3-hexene (T3H). Actually, the fact that the total amount of double bonds has only slightly decreased in the ${ }^{13} \mathrm{C}$ NMR also supports that an isomerization seems to be the most possible reason for the large concentration change of $\mathrm{C} 3 \mathrm{H}$ shown in Figure 3-5. 


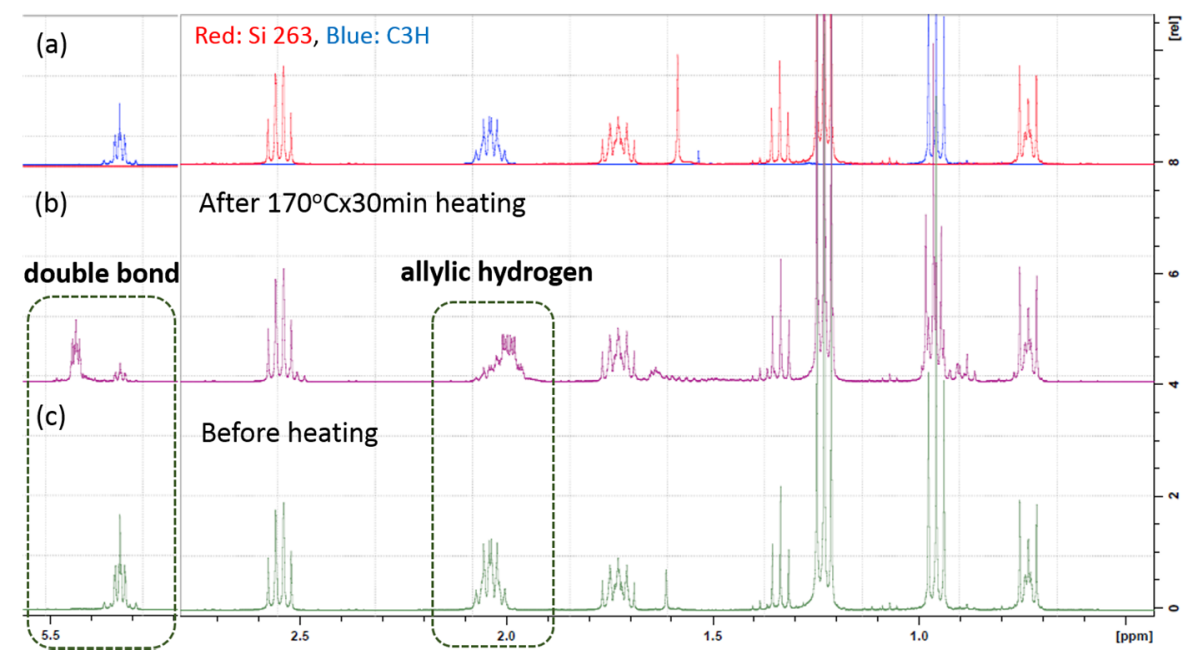

Figure 3-20: ${ }^{1} \mathrm{H}$ NMR of (a) Si 263 and $\mathrm{C} 3 \mathrm{H}$, (b and c) the $\mathrm{C} 3 \mathrm{H} / \mathrm{Si} 263$ mixture after and before heating at $170^{\circ} \mathrm{C}$ for 30 minutes

Since the new peak in the GC/MS measurement has the maximum mass peak of $322 \mathrm{~m} / \mathrm{z}$ which is the same as that in the $3 \mathrm{~m} 1 \mathrm{p}$ system, this implies that the addition reaction of $\mathrm{Si}$ 263 to the $\mathrm{C} 3 \mathrm{H}$ double bond occurs in the same way (Figure 3-21). The detailed ${ }^{13} \mathrm{C} \mathrm{NMR}$ peak analysis clarifies that $\mathrm{T} 3 \mathrm{H}$ is the main component in the sample after heating and confirms an additional small generation of a one to one addition reaction product, as summarized in Figure 3-22 and Table 3-4.
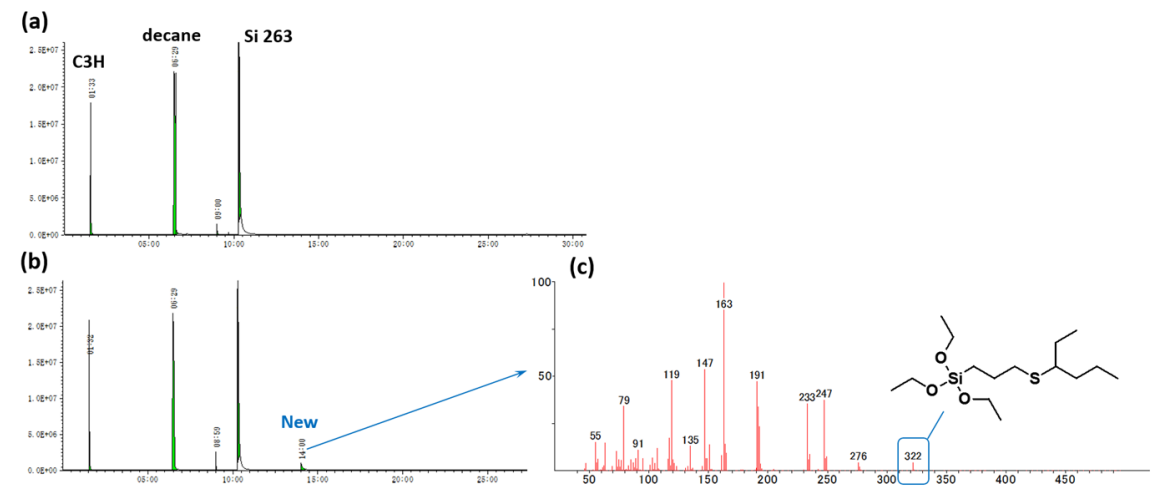

Figure 3-21: GC/MS chromatogram of the C3H / Si 263 / decane mixture (a) before and (b) after heating for 30 minutes at $170^{\circ} \mathrm{C}$, and (c) Mass chromatogram of the peak at retention time14.1 minutes 

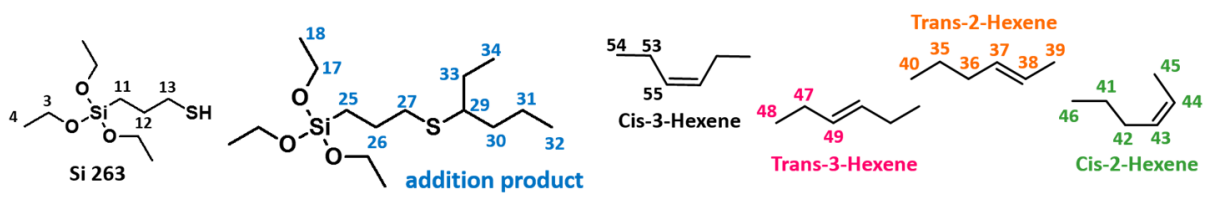

3,17

(b) After heating at $170^{\circ} \mathrm{C}$ for $30 \mathrm{~min}$

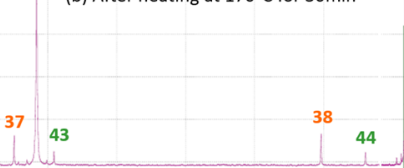

(a) Before heating

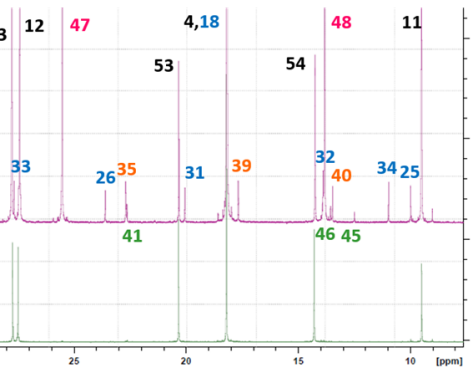

Figure 3-22: ${ }^{13} \mathrm{C}$ NMR of the $\mathrm{C} 3 \mathrm{H} / \mathrm{Si} 263$ mixture (a) before and (b) after heating at $170{ }^{\circ} \mathrm{C}$ for 30 minutes

Table 3-4: Assignment of ${ }^{13} \mathrm{C}$ NMR chemical shifts of reaction products in the $\mathrm{C} 3 \mathrm{H} / \mathrm{Si} 263$ system with calculated chemical shifts

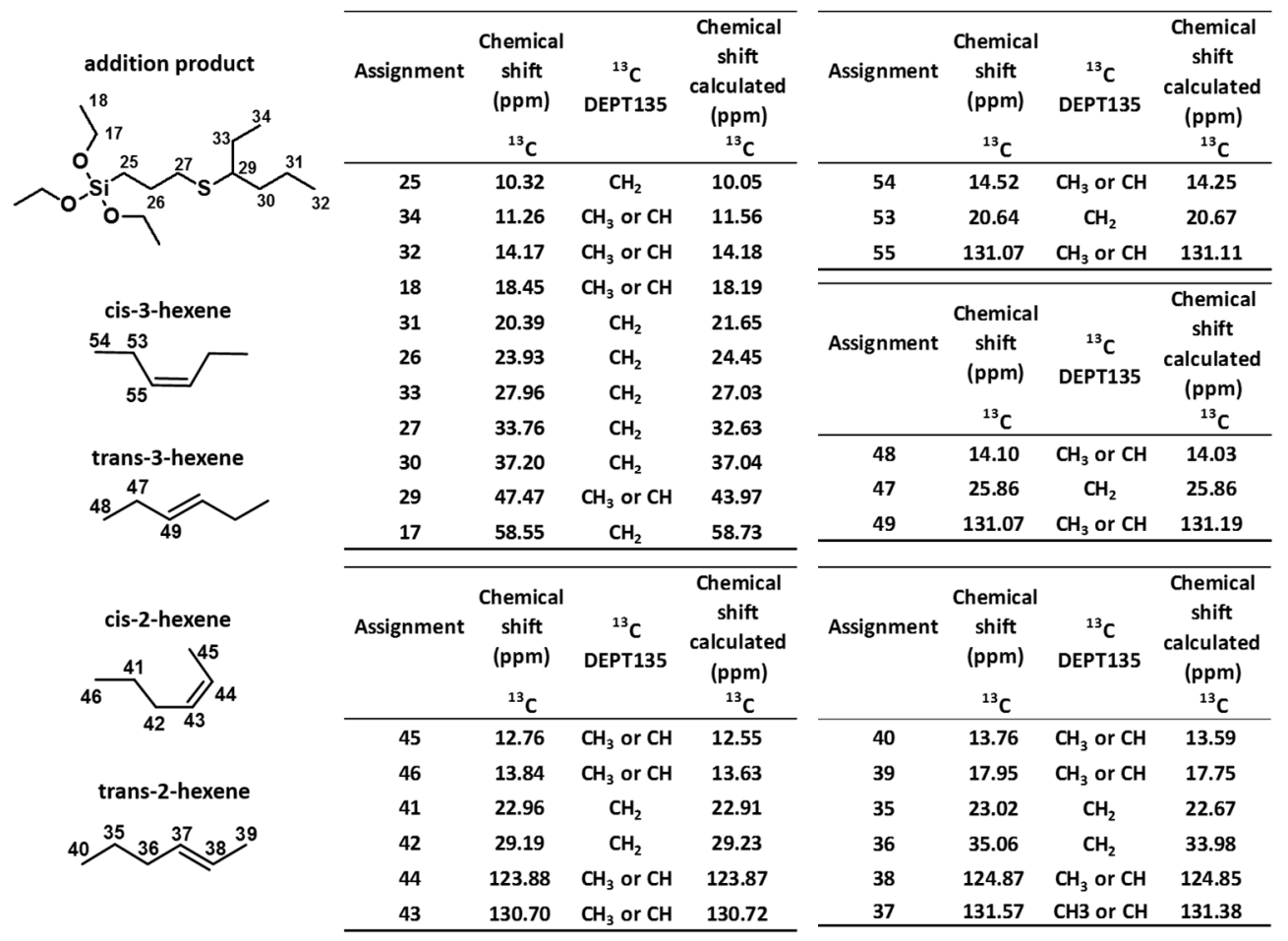


Furthermore, the generation of cis-2-hexene and trans-2-hexene is also observed, which is based on the shift of the position of the double bond. The possible mechanism in the system is assumed to be similar as already discussed in the $3 \mathrm{~m} 1 \mathrm{p}$ system, following again a radical pathway. Firstly, similar to that in the $3 \mathrm{~m} 1 \mathrm{p}$ and Ocd systems, the formed mercaptosilane radicals should be able to attack to the double bond of $\mathrm{C} 3 \mathrm{H}$. Because of the reversibility of this reaction, some parts of the intermediate radicals reacts further resulting in addition products by abstracting the hydrogen from other mercapto-silanes. Other parts of them reacts back to the state of the mercapto-silane and the olefin. During the presence as an intermediate (thioether and carbon centered) radical, this radical should be able to isomerize from cis to trans structure. However, in this system, different isomers are also formed. Besides the cis / trans isomerization also a shift of the double bond to cis-2-hexene and trans-2-hexene is observed. This fact leads to the conclusion that mercapto-silane radicals can also abstract the allylic hydrogen from $\mathrm{C} 3 \mathrm{H}$, which results in the generation of allylic carbon radicals. It is known from the literature that mercapto-silane radicals can abstract a hydrogen from a C-H bond, as well.[15] Since this olefin radical can be delocalized because of the neighbored double bond, the isomerization from cis to trans, and also the shift of the double bond can occur easily as shown in Figure 3-23, which results in the generation of three types of isomers. The higher concentration of allylic hydrogen in the $\mathrm{C} 3 \mathrm{H}$ system compared to that in the $3 \mathrm{~m} 1 \mathrm{p}$ system together with a delocalization effect of generated allylic carbon radical, which subsequently results in the formation of a trans isomer, seems to be the reason of the higher possibility of the allylic hydrogen abstraction in the $\mathrm{C} 3 \mathrm{H}$ system. Therefore, it can be assumed that large amounts of carbon radicals have been generated in the system.

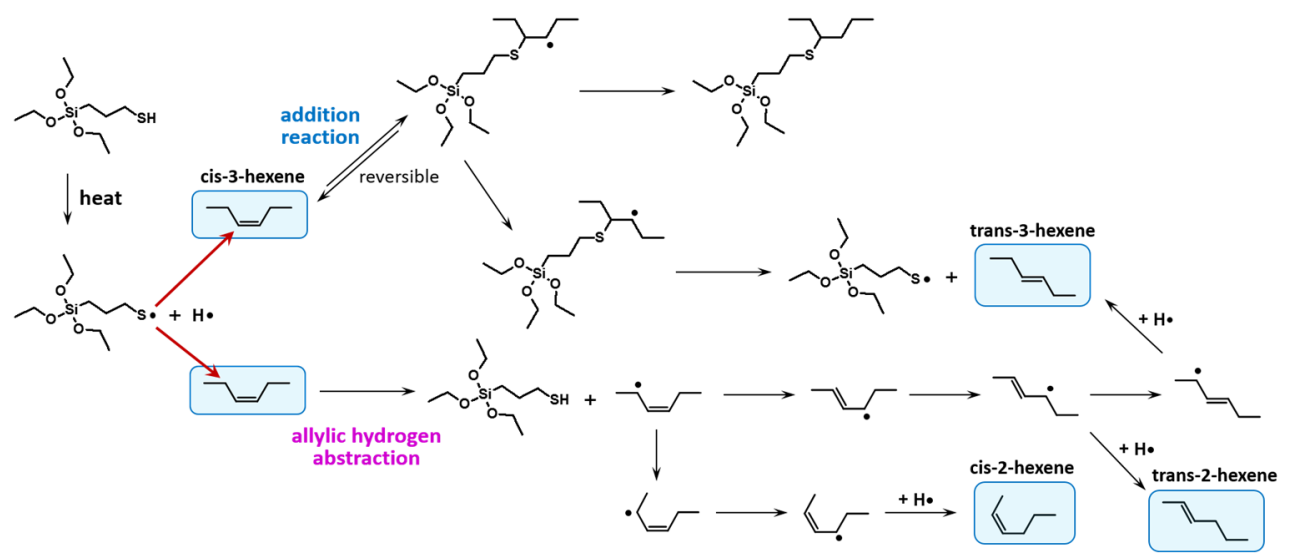

Figure 3-23: Possible reaction mechanism inside C3H / Si 263 mixture 
Figure 3-24 shows the change of concentration ratio of each olefin measured in ${ }^{13} \mathrm{C} N M R$ by using decane as an internal standard at different temperatures. Only a small isomerization is observed at $130^{\circ} \mathrm{C}$, however this isomerization rate increases drastically at $150{ }^{\circ} \mathrm{C}$. On one hand the concentration of trans-2-hexene and cis-2-hexene increases gradually with increasing heating time, on the other hand the ratio between cis-3-hexene and trans-3-hexene seems to end up at ca.70 and $15 \%$ as a concentration ratio in the sample at both 150 and $170^{\circ} \mathrm{C}$.
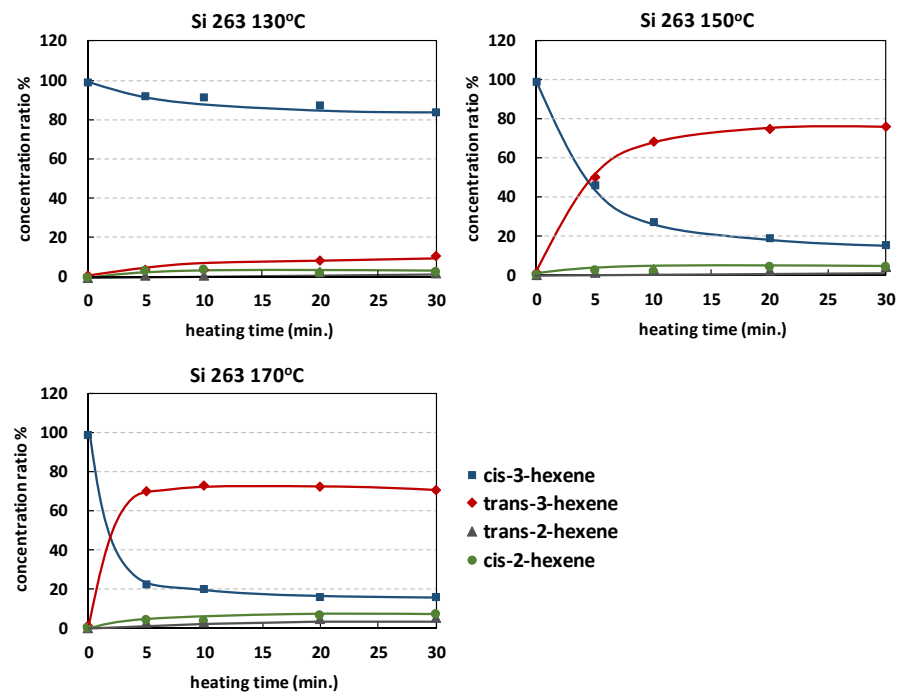

Figure 3-24: Change of concentration ratio of each olefin at different temperatures in the $\mathrm{C} 3 \mathrm{H}$ system

\section{3-3-2-3 T3H / Si 263 system}

trans-3-hexene (T3H)<smiles>CC/C=C/CC</smiles>

Figure 3-25 shows a similar plot in the $\mathrm{T} 3 \mathrm{H}$ where additionally decane was added as it was already depicted in Figure 3-24. Almost no change of the T3H concentration is observed up to $150{ }^{\circ} \mathrm{C}$, and only $7 \%$ of change from the trans to cis structure at $170{ }^{\circ} \mathrm{C}$ after heating for 30 minutes. These results confirm that the trans structure is more stable than the cis structure, therefore, it is more unlikely that an addition reaction and an allylic hydrogen abstraction from a mercapto-silane radical occur. However, when the experiment was carried in the system without decane, the T3H / Si 263 sample after heating at $170^{\circ} \mathrm{C}$ for 30 min gave a different result. The ${ }^{13} \mathrm{C}$ NMR spectrum of the sample was identical to that of the C3H / Si 263 system with same heating history, as shown in Figure 3-26. The addition 
product between Si 263 and T3H and all four types of isomers have been detected with the same concentration as observed in the $\mathrm{C} 3 \mathrm{H} / \mathrm{Si} 263$ system. The increased contact possibility between $\mathrm{T} 3 \mathrm{H}$ and Si 263 due to the removed solvent seems to be the reason for this difference. Although it cannot be finally judged if the addition reaction occurred as one-step reaction mechanism directly from the trans structure or if it proceeded only with the cis structure after the isomerization occurred as two-step mechanism. However, as indicated in the $\mathrm{C} 3 \mathrm{H}$ system, since the equilibrium state between cis and trans is reached at a higher ratio of trans, it is assumed that starting from cis structure leads to a higher amount of generation of radical species in the system until it reaches to the equilibrium condition.
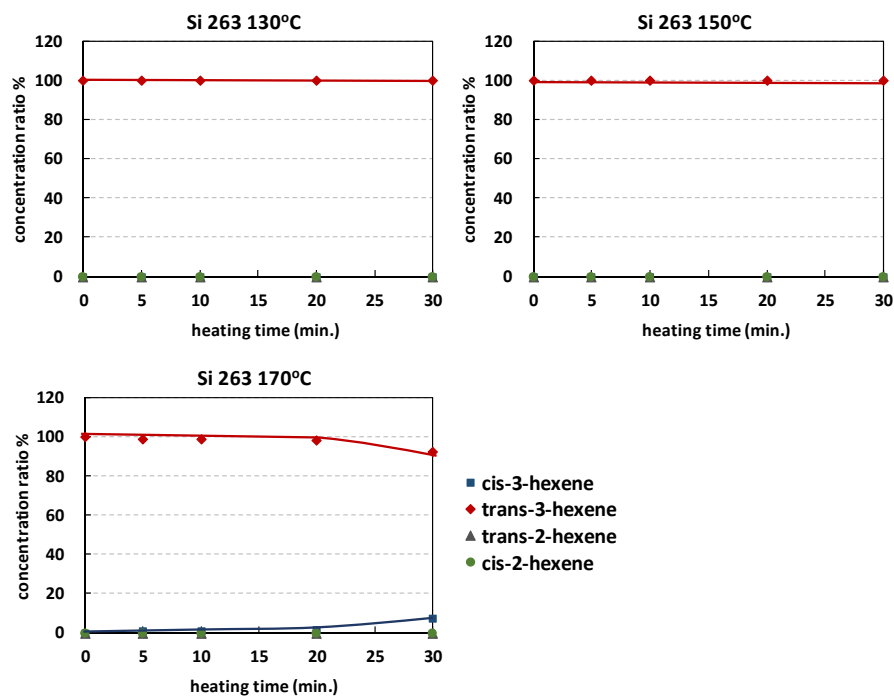

cis-3-hexene

- trans-3-hexene

trans-2-hexene

- cis-2-hexene

Figure 3-25: Change of concentration ratio of each olefin at different temperatures in the T3H system

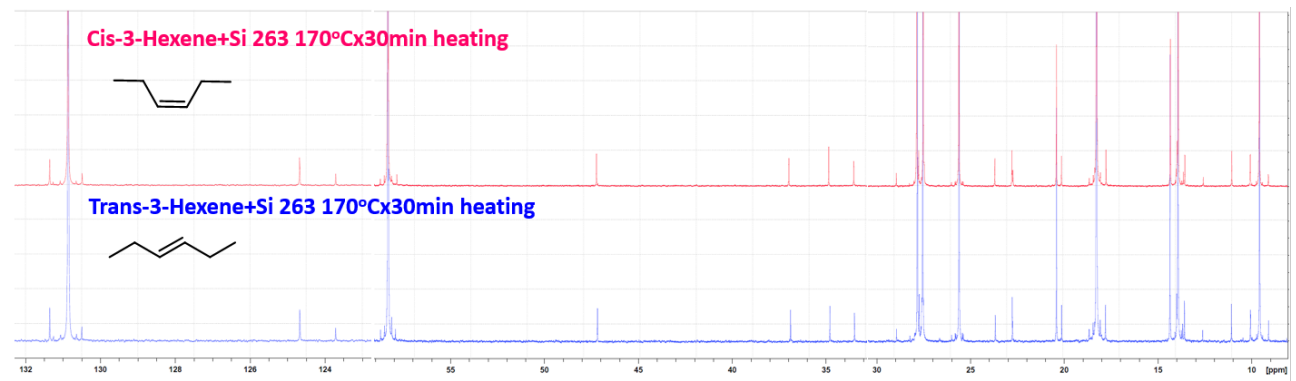

Figure 3-26: ${ }^{13} \mathrm{C}$ NMR of (a) the $\mathrm{C} 3 \mathrm{H} / \mathrm{Si} 263$ mixture and (b) the T3H / Si 263 mixture after heating at $170{ }^{\circ} \mathrm{C}$ for 30 minutes 


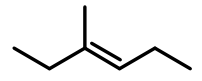

In order to understand the influence of a methyl group on the double bond, a further investigation in the trans-3-methyl-3-hexene / Si 263 was carried out. Using the cis structure of this molecule would have been better to have a more similar structure to natural and isoprene rubber, but due to the limitation of the commercial availability of this chemical, the investigation was conducted by using the trans structure. Furthermore, the peak analysis of the ${ }^{13} \mathrm{C}$ NMR spectrum confirmed that the sample originally contains $14 \%$ of $\mathrm{C} 3 \mathrm{M} 2 \mathrm{H}$ which is an isomer of $\mathrm{T} 3 \mathrm{M} 3 \mathrm{H}$ (Figure 3-28).

Figure 3-27 shows the ${ }^{1} \mathrm{H}$ NMR spectra of the T3M3H / Si 263 mixture without decane before and after heating at $170{ }^{\circ} \mathrm{C}$ for $30 \mathrm{~min}$. The change of the peak shape around 2.5 ppm, which is characteristic of the addition reaction of Si 263 to the double bond, is not observed in a significant amount. While, the changes of the peak shapes corresponding to an allylic hydrogen and to a double bond are visible. The same as inside the previous systems, the identification of all of peaks before and after heating in the ${ }^{13} \mathrm{C} N M R$ spectrum was carried out (Figure 3-28). Moreover, three isomers which are $\mathrm{C} 3 \mathrm{M} 3 \mathrm{H}, \mathrm{T} 3 \mathrm{M} 2 \mathrm{H}$ and $\mathrm{T} 4 \mathrm{M} 2 \mathrm{H}$ are identified only in the sample after heating. However, no addition reaction products of Si 263 to the double bond of olefin are detected.

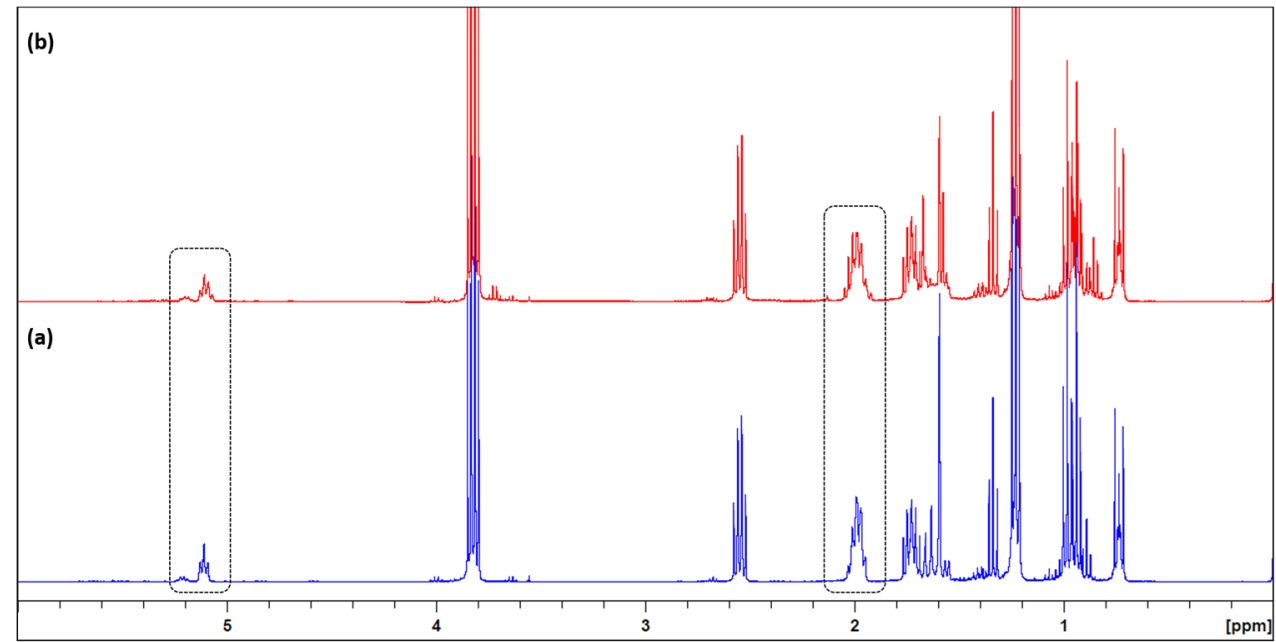

Figure 3-27: ${ }^{1} \mathrm{H}$ NMR of the T3M3H / Si 263 mixture (a) before and (b) after heating at 170 ${ }^{\circ} \mathrm{C}$ for 30 minutes 


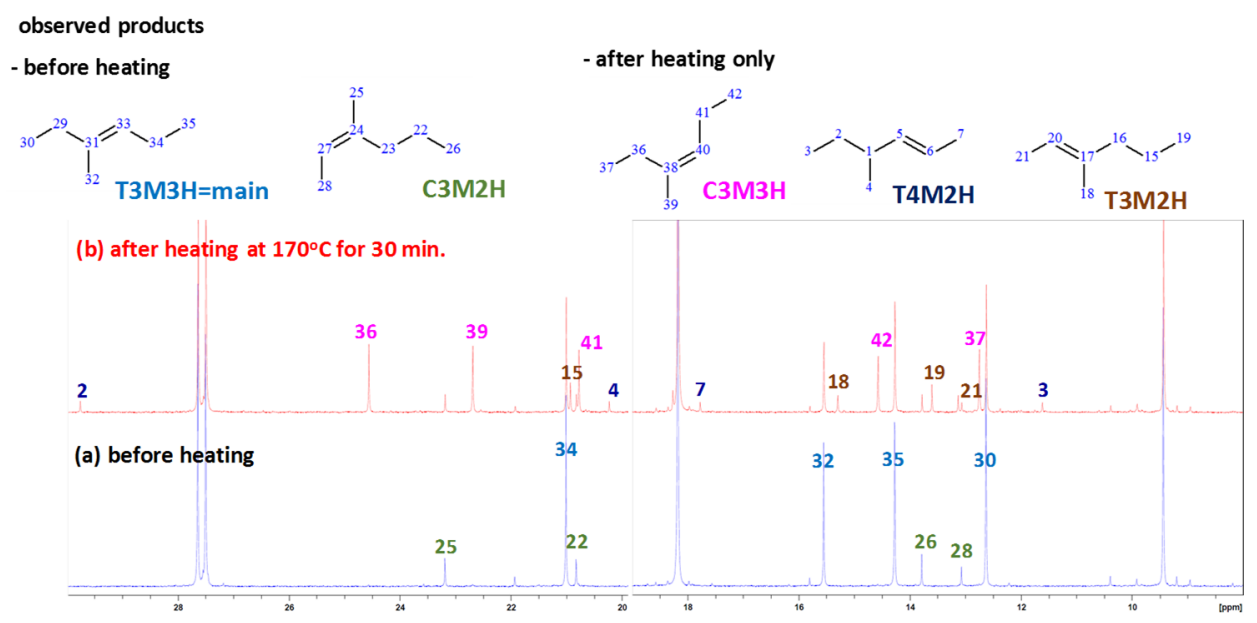

(b) after heating at $170^{\circ} \mathrm{C}$ for $30 \mathrm{~min}$.

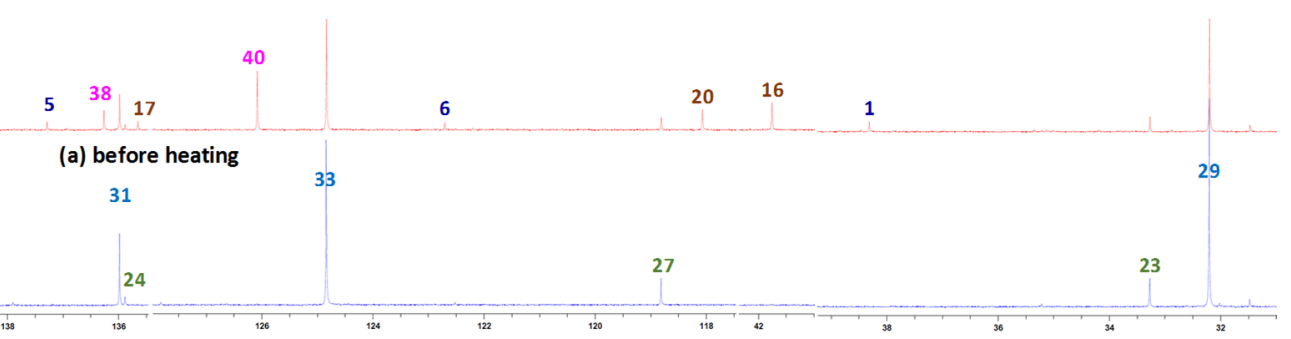

Figure 3-28: ${ }^{13} \mathrm{C}$ NMR of the T3M3H / Si 263 mixture (a) before and (b) after heating at 170 ${ }^{\circ} \mathrm{C}$ for 30 minutes

Figure 3-29 shows the results of the change of the concentration ratio of each isomer by using decane as an internal standard at different temperatures. Compared to the T3H system, which does not contain any methyl group at the double bond, the isomerization degree is more pronounced in the $\mathrm{T} 3 \mathrm{M} 3 \mathrm{H}$ system at all temperatures. In the case of the $\mathrm{T} 3 \mathrm{M} 3 \mathrm{H}$ structure, the generated radical by the allylic hydrogen abstraction can be transformed to a tertiary radical by the delocalization effect. A tertiary radical is known to be more stable than a secondary radical.[19] This higher stabilization of the created radical results in the easiness of the allylic hydrogen abstraction. It is reasonable to assume that this is the reason for a more pronounced isomerization in the $\mathrm{T} 3 \mathrm{M} 3 \mathrm{H}$ system compared to the T3Hs system where only secondary radicals can be generated. 

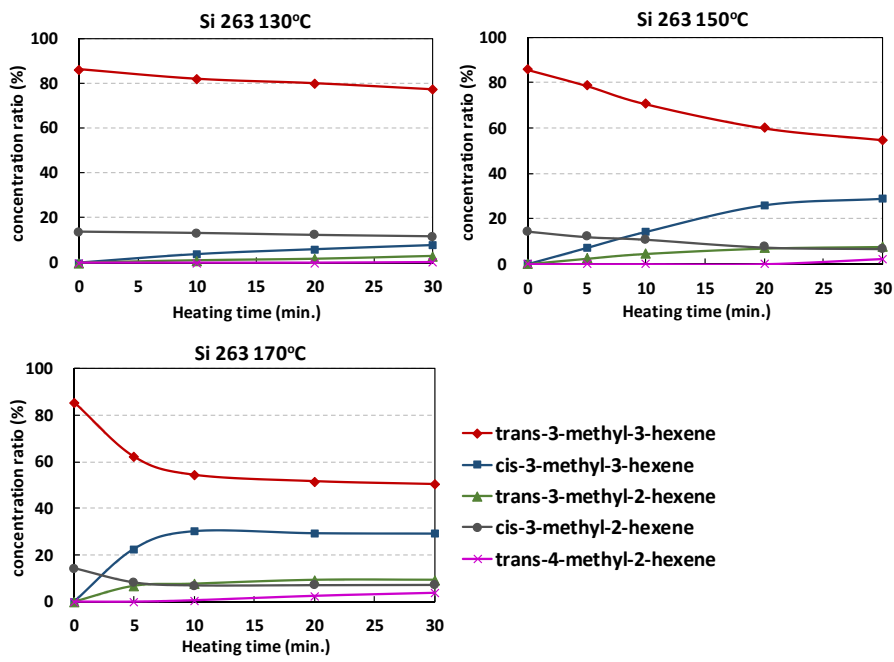

Figure 3-29: Change of concentration ratio of each olefin at different temperatures in the T3M3H system

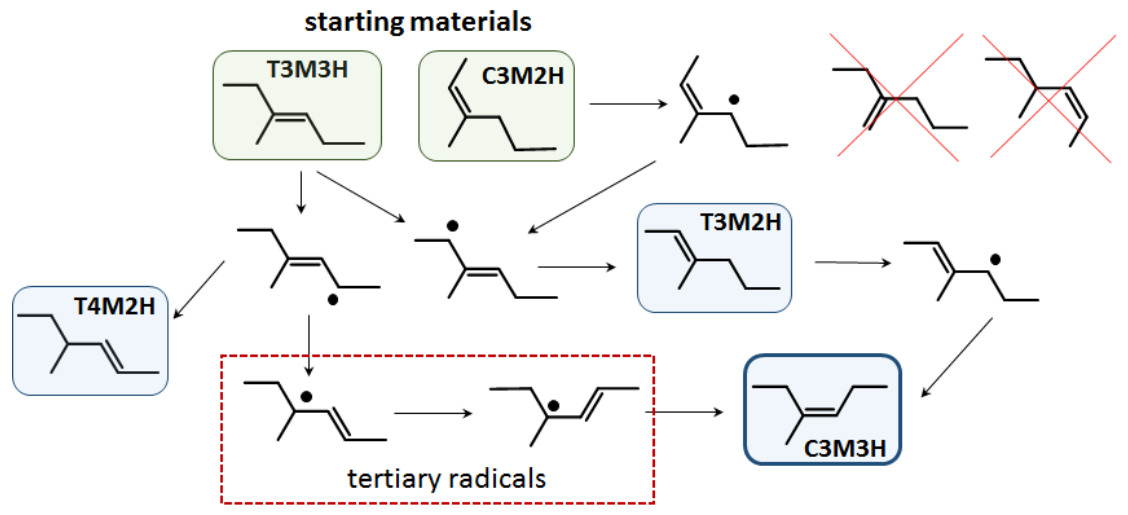

Figure 3-30: Possible isomerization mechanism for an allylic hydrogen abstraction in T3M3H system

The fact that though the addition reaction product between Si 263 and T3H was detected in the sample without decane, but was not observed in the T3M3H system even in the sample without decane is an interesting point. The double bond in T3M3H has a higher stability by the electron donation effect of the methyl group than that in $\mathrm{T} 3 \mathrm{H}$. A combination effect of this and the above mentioned higher stability of the tertiary radical seem to give the system a higher priority to the allylic hydrogen abstraction compared to the addition 
reaction. This implies that the mercapto-silane could also behave differently in SBR or BR and NR or IR in the rubber compounds. However, it is worth to add that the new peak which has a molecular weight corresponding to the mass value of the addition reaction product between Si 263 and T3M3H is observed only in the GC/MS measurement. Therefore, it seems that a small amount of it is produced, though it was below the detection limit in the ${ }^{13} \mathrm{C}$ NMR measurement. This would lead to the consideration as following that the addition reaction is not impossible to the isoprene type of the double bond, therefore this reaction might occur as well in the rubber compound system.

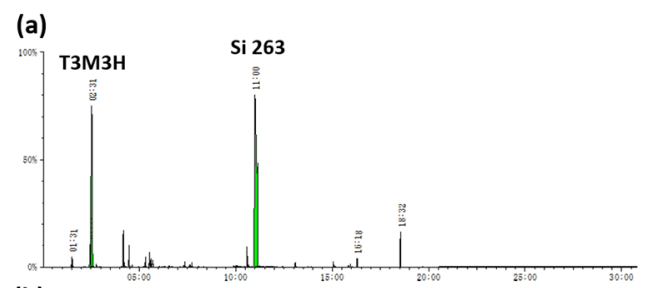

(b) (c)

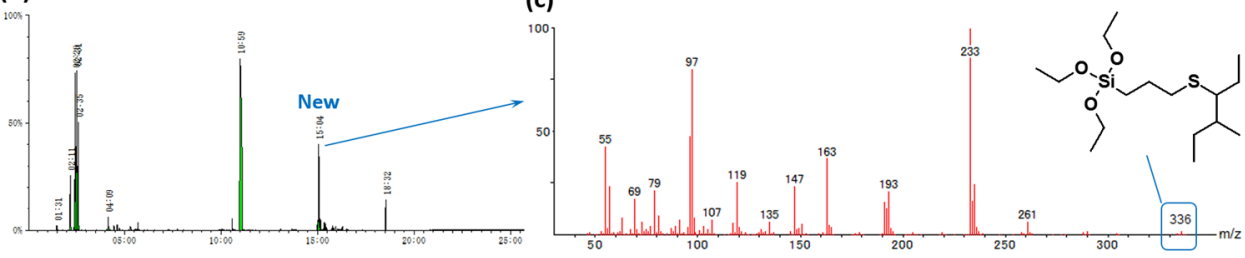

Figure 3-31: GC/MS chromatogram of the T3M3H / Si 263 mixture (a) before and (b) after heating for 30 minutes at $170^{\circ} \mathrm{C}$, and (c) Mass chromatogram of the peak at retention time15.0 minutes

\section{3-3-2-5 TME / Si 263 system}

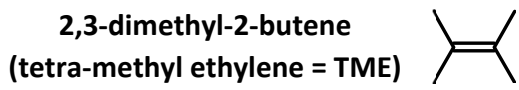

Lastly, using TME which has four equivalent methyl groups on the double bond, same experiments as for other olefins so far was carried put.

In this system, only a very small difference can be observed in ${ }^{1} \mathrm{H}$ NMR spectra between before and after heating at $170{ }^{\circ} \mathrm{C}$ for $120 \mathrm{~min}$. Only $7 \%$ of 2,3-dimethyl-1-butene, which is an isomer of TME shown in Figure 3-32, is observed as a new resulting product in the ${ }^{13} \mathrm{C}$ NMR spectrum of the same sample. A small amount of the addition reaction product is observed in the GC/MS measurement but not in the ${ }^{13} \mathrm{C} N M R$, which is the same as in the $\mathrm{T} 3 \mathrm{M} 3 \mathrm{H}$ system. The double bond of this olefin has the highest stability in all investigated olefins. Therefore, the addition reaction does not occur easily, and even if the allylic 
hydrogen atom is abstracted, the generated TME radical prefers to react back to the original structure because of the higher stability of TME.

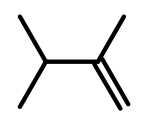

Figure 3-32: Observed isomer of TME after heating

\section{3-3-2-6 Summary of the model study with Si 263}

By heating the mixture of six different olefins with Si 263 , the reaction products, the rate and the mechanism were investigated. For the quantification of each component, a sample which contains decane as an internal standard was used.

It was clarified based on the structure of the resulting products that mercapto-silanes react with olefins following a radical pathway. Even without a radical initiator, mercaptosilane radicals can be generated through the dissociation of the $\mathrm{S}-\mathrm{H}$ bond by heating or a molecular assisted hemolysis. They affect olefins in the following two ways.

The first one is the reversible addition reaction to the double bond of olefins. Because of this reaction, addition reaction products or isomers can be produced. The easiness of the addition reaction depends on the stability of the double bond in the olefin, and the resulting ratio of the addition reaction product to the original olefin and the isomers is determined by the stability balance of those structures.

Another way is the abstraction of the allylic hydrogen from the olefin. This reaction with the subsequent radical transfer results in the generation of the double bond shifted isomers. It can be considered that when the stability of the double bond in the original olefin or the generated radical by the allylic hydrogen abstraction is high, the abstraction can occur easily with higher priority compared to the addition reaction.

In the case of $3 \mathrm{~m} 1 \mathrm{p}$ and Ocd which have terminal double bonds, addition reaction products with the mercapto-silane at the end of the double bond (Anti-Markovnikov structure) were detected with the largest amount among the evaluated olefins. This reaction was confirmed to proceed even at $110{ }^{\circ} \mathrm{C}$ and the reaction rate increased 
significantly at $150^{\circ} \mathrm{C}$. As the terminal double bond is very reactive, the addition reaction can occur easily, and the equilibrium state is more on the side of the addition reaction product.

In the case of olefins which have an internal double bond, the addition reaction product between $\mathrm{C} 3 \mathrm{H}$ and Si 263 was observed in the ${ }^{13} \mathrm{C}$ NMR spectrum of the sample with decane, however the detected amount of it after 30 minutes heating at $150{ }^{\circ} \mathrm{C}$ was only one-fifth relative to that in the $3 \mathrm{~m} 1 \mathrm{p}$ system. The main reaction products were isomers, and not only $\mathrm{T} 3 \mathrm{H}$ but also the double bond shifted isomers such as $\mathrm{T} 2 \mathrm{H}$ and $\mathrm{C} 2 \mathrm{H}$ were obtained. The main mechanism of the isomerization from $\mathrm{C} 3 \mathrm{H}$ to $\mathrm{T} 3 \mathrm{H}$ seems to be the reversible addition reaction of the mercapto-silane radical to the cis double bond. It seems that T3H remains as a main component in the system after heating because of the higher stability of T3H than that of $\mathrm{C} 3 \mathrm{H}$ and the addition reaction product. Furthermore, it seems reasonable to consider that as the stability of the double bond in $\mathrm{C} 3 \mathrm{H}$ is higher than that in $3 \mathrm{~m} 1 \mathrm{p}$, the mercapto-silane radical can abstract an allylic hydrogen from $\mathrm{C} 3 \mathrm{H}$ as well. As a result of the delocalization of the generated allylic radicals, $\mathrm{C} 2 \mathrm{H}$ and $\mathrm{T} 2 \mathrm{H}$ can be produced because of the similar stability of them with $\mathrm{C} 3 \mathrm{H}$ and $\mathrm{T} 3 \mathrm{H}$, respectively. Actually, it has been reported in the research where the quantitative determination of vulcanizates of 3-hexene (bis(alkenyl)sulfides) was conducted, both of the addition reaction structures in the allylic position of 3-hexene and 2-hexene were detected with a similar concentration.

In the $\mathrm{T} 3 \mathrm{H}$ system using decane as a solvent, the degree of isomerization was lower than that in the $\mathrm{C} 3 \mathrm{H}$ system, and double bond shifted isomers as well as an addition reaction product were not observed in the ${ }^{13} \mathrm{C}$ NMR spectrum. This seems to be because of the following reasons. The higher stability of $\mathrm{T} 3 \mathrm{H}$ compared to $\mathrm{C} 3 \mathrm{H}$ has resulted in a higher difficulty to be attacked by the mercapto-silane radical, and also even if the addition reaction occurs, the intermediate radical (mercapto-silane added carbon radical) tends to react back to the original $\mathrm{T} 3 \mathrm{H}$ structure because of the higher stability of it compared to $\mathrm{C} 3 \mathrm{H}$ and the addition reaction product. However, when T3H and Si 263 were heated without decane at $170{ }^{\circ} \mathrm{C}$ for 30 minutes, the resulting solution showed a same composition ratio of reaction products as that in the $\mathrm{C} 3 \mathrm{H}$ system. This seems to be because of the increased contact possibility between $\mathrm{T} 3 \mathrm{H}$ and a mercapto-silane radical, which resulted in the higher driving force to move to the equilibrium state. 
In the $\mathrm{T} 3 \mathrm{M} 3 \mathrm{H}$ system which has a methyl group at the trans double bond, on one hand the addition reaction product between it and a mercapto-silane radical was not observed even without decane in the ${ }^{13} \mathrm{C}$ NMR spectrum but observed only in the GC/MS spectrum. This indicates that the concentration of the existing addition reaction product after heating is very low. On the other hand, the isomerization rate of $\mathrm{T} 3 \mathrm{M} 3 \mathrm{H}$ was higher than that of $\mathrm{T} 3 \mathrm{H}$, and double bond shifted isomers were also detected. This mechanism could be explained as following. Because of the higher stability of the double bond compared to that in $\mathrm{T} 3 \mathrm{H}$, the addition reaction by the mercapto-silane radical doesn't occur easily. However, if it occurs, as the created mercapto-silane added carbon radical is a tertiary radical, it has a relatively higher stability which enables to change to the cis structure resulting in the faster isomerization rate. Furthermore, the created allyl carbon radical by the allylic hydrogen abstraction can transform to the stable tertiary radical by the delocalization effect as well. This can be the reason for an easy allylic hydrogen abstraction resulting in the faster generation of double bond shifted isomers compared to the T3H system.

In the case of TME which has four equivalent double bonds, it looks like that only a small amount of isomerization reaction has occurred in the system during heating. It is reasonable to assume that the addition reaction by a mercapto-silane radical was very difficult to occur due to the high stability of the double bond. Furthermore, there seems to be possible that a mercapto-silane radical abstracts allylic hydrogens from TME. However, all possible reactions lead again back to the original TME structure because of the lower stability of the isomer compared to TME.

From above mentioned points, the following reactions could be expected in the mercapto-silane used silica filled rubber compound. Mercapto-silanes are able to react with the rubber double bond directly, especially with vinyl and cis structures, under the mixing conditions at elevated temperature, which lead to the formation of chemical bound rubber in the compounds. In addition, the presence of mercapto-silanes lead to the generation of large amounts of polymer radicals because of the allylic hydrogen abstraction ability of mercapto-silane itself and also the mercapto-silane added intermediate carbon radicals. 


\section{3-3-3 Si 69 systems}

In this section, the results in Si 69 systems will be summarized. It is described in the literature that in the case of $\mathrm{Si} 69$ silica filled rubber compounds, the chemical bound rubber is already created at the $1^{\text {st }}$ mixing stage which doesn't contain any curatives.[20-22] This indicates that, at least, a part of silanes have already reacted with the silica surface and have formed as well a chemical bond to the polymer.

Based on the literature, the following reaction mechanisms shown in Figure 3-33 could be considered. The first one is the formation of cross-linked products by the active sulfur species released from the long sulfur chain of Si 69 by heating. Actually, by heating the mixture of only TME and Si 69, the cross-linked products (A) with different sulfur chain lengths, where sulfur couples at the allylic carbon of TME and combines two molecules of TME, has been proposed as reaction products in previous studies, as shown in Figure 3-33 (A). $[8,9,20,21]$ Furthermore, considering the already reported structure of cross-linked products in the unaccelerated sulfur vulcanization, [23-25] the structure where sulfur added at a double bond of an olefin seems to be produced as well (structure (B)).[23-27] The $2^{\text {nd }}$ possible reaction is a reaction of the dissociated silane radical ( $(\mathrm{EtO})_{3} \mathrm{Si}_{(}\left(\mathrm{CH}_{2}\right)_{3} \mathrm{~S}_{\mathbf{x}} \bullet$, persulfenyl radical) thermally to an olefin. In this case, two routes can be considered as pathways regarding how this persulfenyl radical adds to an olefin. One of them is a radical AntiMarkovnikov addition reaction, which was similarly observed in the Si 263 system (structure (C)). However, because of a relatively higher stability of the persulfenyl radical, no evidence that the radical reacts with an olefin double bond directly has been reported in the literature. Instead of that, in the investigation of the reaction between dibenzyl tetrasulfide and 2methyl-2-pentene, the reaction pathway that the persulfenyl radical obtains a hydrogen and the formed $\mathrm{RS}_{\mathrm{x}} \mathrm{H}$ structure has a Markovnikov type of addition reaction to an olefin has been reported as a second route.[28] The proton in perthiols, $\mathrm{RS}_{\mathrm{x}} \mathrm{H}$, is expected to be much more acidic than this in thiols, and thus an electrophilic Markovnikov addition to the double bond would explain the formation of the structure (C) as well. Furthermore, at the same time, there is also the evidence that the $\mathrm{RS}_{\mathrm{x}}$ component reacts at the allylic site of 2-methyl2-pentene resulting in the allylic addition reaction products in the same study, which corresponds to the structure (D) in the case of Si 69. Therefore, two structures of addition reaction products can be considered. In the $\mathrm{T} 3 \mathrm{H}$ and Si 69 system, the addition reaction products of $(\mathrm{EtO})_{3} \mathrm{Si}\left(\mathrm{CH}_{2}\right)_{3} \mathrm{~S}_{\mathrm{x}}$ part from $\mathrm{Si} 69$ at the allylic carbon of $\mathrm{T} 3 \mathrm{H}$ has been proposed 
in the literature, however, the addition site has not been specified exactly because the used data to assume the structure was only mass data obtained in the LC/MS measurement.[10]<smiles></smiles><smiles>[R]C/C=C\CC[R]</smiles>

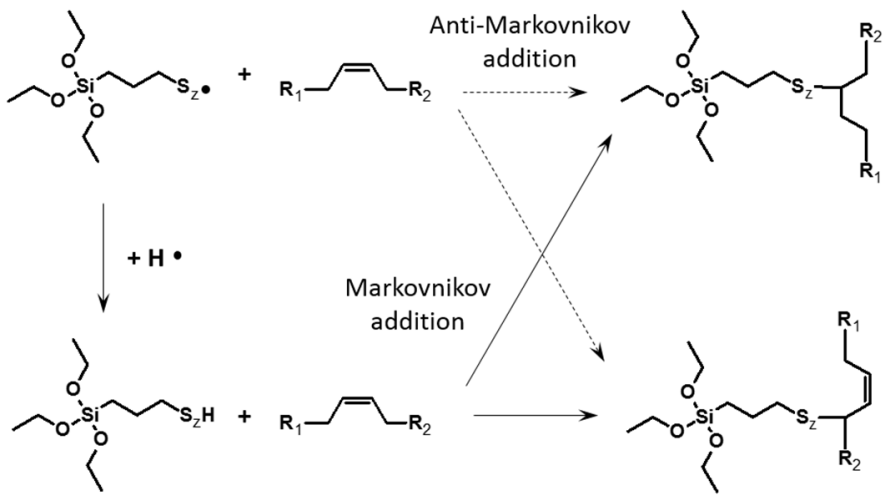

(D)

Figure 3-33: Possible reaction mechanism between Si 69 and an olefin (The generation of reaction product $(A)$ in the case of TME and $(D)$ in the case of T3H has been proposed in the literature $[8-10,20,21]$ ) (number of sulfur atom can be variable)

Based on the assumptions so far, the reaction of Si 69 with some olefins was investigated, respectively.

\section{3-3-3-1 TME / Si 69 system}

Firstly, the results in the TME /Si 69 system are compared with the results which were already described in the literature.[8,9,20,21] Since the conversion of TME after heating together with Si 69 was not as high as in the Si 263 system, more emphasis was put on the 
clarification of the structure of the reaction products which could be produced by extending the heating time at higher temperature $\left(170^{\circ} \mathrm{C}\right)$.

When the heating time is extended to 1 hour or 5 hours at $170{ }^{\circ} \mathrm{C}$, some changes in the ${ }^{1} \mathrm{H}$ NMR spectra are observed as shown in Figure 3-34. Firstly, it was evaluated whether the cross-linked product shown in Figure 3-33 (A) was generated. The calculated chemical shifts of the products at ${ }^{1} \mathrm{H}$ NMR and ${ }^{13} \mathrm{C}$ NMR are summarized in Table 3-5. The signal of the proton atom at the carbon atom (1) next to the sulfur atom of the structure (A) should appear in the chemical shift range between 3.52 and $3.63 \mathrm{ppm}$ depending on the sulfur length, and all other methyl protons should be observed between 1.62 and $1.79 \mathrm{ppm}$. As highlighted in the Figure 3-34 as zoom 1 and 2, small changes in both chemical shift ranges by heating can be observed in the ${ }^{1} \mathrm{H}$ NMR spectra.

Table 3-5: Calculated chemical shift of sulfur cross-linked TME products in NMR measurements

\begin{tabular}{|c||c|c||}
\hline & Proton (ppm) & Carbon (ppm) \\
\hline \hline$(1)$ & $3.52-3.63$ & $42.76-44.10$ \\
\hline$(2)$ & - & $130.28-132.02$ \\
\hline$(3)$ & $1.62-1.79$ & $18.20-18.68$ \\
\hline$(4)$ & - & $122.20-122.70$ \\
\hline$(5)$ & $1.62-1.79$ & $20.90-21.20$ \\
\hline$(6)$ & $1.62-1.79$ & $20.90-21.20$ \\
\hline
\end{tabular}

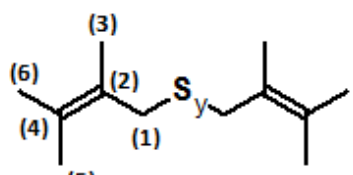

(5)

The results of ${ }^{13} \mathrm{C}$ NMR and ${ }^{13} \mathrm{C}$ DEPT135 measurements, which were carried out further to specify the reaction product structure, are summarized together with the calculated values of some possibly involved products in the system in Table $3-6 .{ }^{13} \mathrm{C}$ NMR spectrum of the sample after heating shows many newly appeared small peaks beside the signals for the starting materials (TME and Si 69). Relatively large peaks are observed in all chemical shift ranges which are picked up in Table 3-5 as peaks of structure (A). Furthermore, the fact that a correlation between the carbon atom (42-44 ppm) and the proton atom (3.5-3.6 ppm) of the methylene group can be observed in the HSQC spectrum (Figure 3-35) also supports the generation of the cross-linked product $(A)$. 


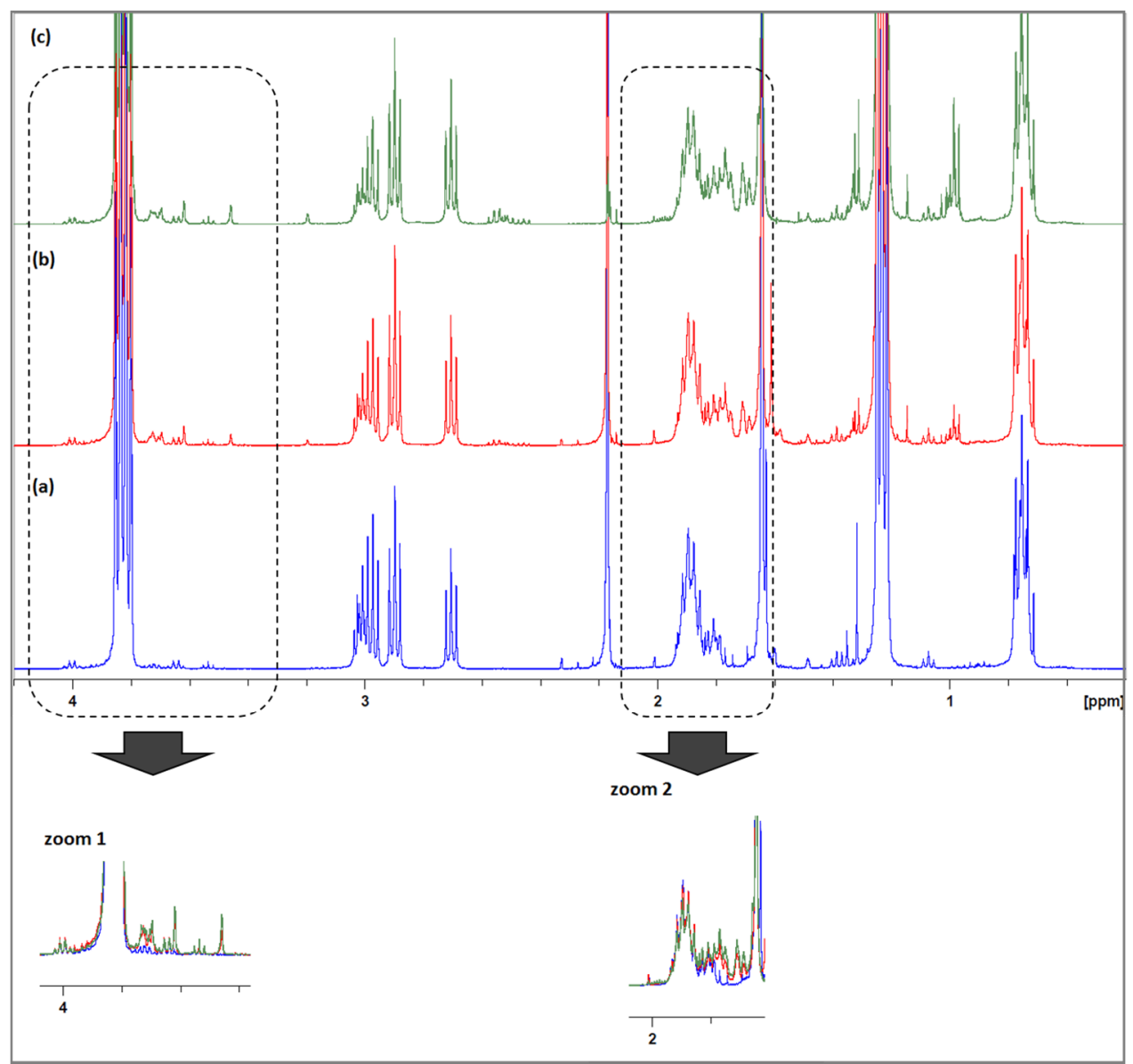

Figure 3-34: ${ }^{1} \mathrm{H}$ NMR spectra of TME / Si 69 mixture; (a) before heating, (b) after heating at $170{ }^{\circ} \mathrm{C}$ for $60 \mathrm{~min}$, (c) after heating at $170{ }^{\circ} \mathrm{C}$ for 5 hours 
Table 3-6: ${ }^{13} \mathrm{C}$ NMR data of TME / Si 69 mixture

\begin{tabular}{|c|c|c|c|c|c|c|c|c|c|c|}
\hline \multicolumn{6}{|c|}{ Calcurated chemical shift, ${ }^{13} \mathrm{C}$ NMR } & Ref. $\left[{ }^{*}\right]$ & \multirow{2}{*}{\multicolumn{4}{|c|}{$\begin{array}{c}\text { Actual chemical shift, }{ }^{13} \mathrm{C} \text { NMR } \\
\text { Sample }\end{array}$}} \\
\hline \multicolumn{2}{|c|}{ starting material } & \multicolumn{5}{|c|}{ Reaction product option } & & & & \\
\hline TME & $\mathrm{S}_{2}-\mathrm{S}_{5}$ silane & $\begin{array}{c}\text { TME } \\
\text { isomer }\end{array}$ & $\begin{array}{c}\text { TME }+S_{1} \text { silane } \\
\text { (1) } \\
\end{array}$ & $\begin{array}{c}\mathrm{TME}+\mathrm{S}_{1} \text { silane } \\
\text { (2) }\end{array}$ & $T M E+S_{x=2-5}+T M E$ & $\begin{array}{c}\mathrm{TME}+\mathrm{Sx} \\
+\mathrm{TME} \\
\end{array}$ & \multirow{2}{*}{\begin{tabular}{|c|}
$\begin{array}{c}\text { TME }+ \text { Si } 69 \\
\text { no heating }\end{array}$ \\
9.68 \\
\end{tabular}} & \multirow{2}{*}{$\begin{array}{c}\text { TME+Si } 69 \\
170^{\circ} \mathrm{C} \times 60 \mathrm{~min} \\
9.68\end{array}$} & $\begin{array}{c}\text { TME+Si } 69 \\
170^{\circ} \mathrm{C} \times 5 \text { hours } \\
\end{array}$ & DEPT135 \\
\hline & \begin{tabular}{rr|}
9.65 & \\
-9.75 & $\mathrm{CH}_{2}$
\end{tabular} & & (11) $9.87 \quad \mathrm{CH}_{2}$ & & & & & & 9.68 & $\mathrm{CH}_{2}$ \\
\hline & & & & (11) $10.19 \mathrm{CH}_{2}$ & & & - & - & 10.51 & $\mathrm{CH}_{2}$ \\
\hline & & & & & & & - & - & 14.53 & $\mathrm{CH}$ or $\mathrm{CH}_{3}$ \\
\hline & & & & $(17) 17.66 \quad \mathrm{CH}_{3}$ & & & - & 17.82 & 17.82 & $\mathrm{CH}$ or $\mathrm{CH}_{3}$ \\
\hline & $18.19 \quad \mathrm{CH}_{3}$ & & $\begin{array}{cc:c}(4,7,10) & & \\
18.19 & \mathrm{CH}_{3} & \end{array}$ & $\begin{array}{cc}(4,7,10) & \mathrm{CH}_{3} \\
18.19 & \end{array}$ & $\begin{array}{r}(3) 18.20 \\
-18.68\end{array} \mathrm{CH}_{3}$ & 18.20 & 18.39 & 18.40 & $18.20,18.39$ & $\mathrm{CH}$ or $\mathrm{CH}_{3}$ \\
\hline & & $20.00 \quad \mathrm{CH}_{3}$ & & & & & - & 20.12 & 20.12 & $\mathrm{CH}$ or $\mathrm{CH}_{3}$ \\
\hline \multirow[t]{31}{*}{$20.38 \quad \mathrm{CH}_{3}$} & & & $\begin{array}{c:c}(17,20) & \mathrm{CH}_{3} \\
20.29 & \end{array}$ & & & & 20.38 & 20.38 & 20.38 & $\mathrm{CH}$ or $\mathrm{CH}_{3}$ \\
\hline & & & & $(19) 21.00 \quad \mathrm{CH}_{3}$ & (6)20.90- $\mathrm{CH}_{3}$ & 20.90 & - & $20.75-21.02$ & $20.75-21.02$ & $\mathrm{CH}$ or $\mathrm{CH}_{3}$ \\
\hline & & & & $(20) 21.05 \quad \mathrm{CH}_{3}$ & $(5)-21.20 \quad \mathrm{CH}_{3}$ & 21.20 & - & (4peaks) & (4peaks) & $\mathrm{CH}$ or $\mathrm{CH}_{3}$ \\
\hline & & $21.50 \quad \mathrm{CH}_{3}$ & & & & & - & 21.50 & 21.50 & $\mathrm{CH}$ or $\mathrm{CH}_{3}$ \\
\hline & & & $\begin{array}{c}(18,19) \\
21.78\end{array} \quad \mathrm{CH}_{3}$ & & & & - & 21.85 & - & - \\
\hline & \begin{tabular}{cc|}
24.42 & $\mathrm{CH}_{2}$ \\
-24.56 &
\end{tabular} & & & & & & $\begin{array}{r}22.50 \\
-22.80\end{array}$ & $22.50-22.80$ & $22.50-22.80$ & $\mathrm{CH}_{2}$ \\
\hline & & & & $(12) 24.52 \quad \mathrm{CH}_{2}$ & & & - & 23.51 & 23.51 & $\mathrm{CH}_{2}$ \\
\hline & & & (12) $24.79 \quad \mathrm{CH}_{2}$ & & & & - & 23.67 & 23.67 & $\mathrm{CH}_{2}$ \\
\hline & & & & & & & - & 24.96 & 24.96 & $\mathrm{CH}$ or $\mathrm{CH}_{3}$ \\
\hline & & & & & & & - & 25.18 & 25.18 & $\mathrm{CH}$ or $\mathrm{CH}_{3}$ \\
\hline & & & & & & & - & 26.03 & 26.03 & $\mathrm{CH}$ or $\mathrm{CH}_{3}$ \\
\hline & & & & & & & - & 26.44 & 26.44 & $\mathrm{CH}$ or $\mathrm{CH}_{3}$ \\
\hline & & & & & & & - & 27.62 & 27.62 & $\mathrm{CH}_{2}$ \\
\hline & & & & & & & - & 27.88 & 27.88 & $\mathrm{CH}_{2}$ \\
\hline & & & $(13) 30.58 \quad \mathrm{CH}_{2}$ & & & & - & 30.75 & 30.75 & $\mathrm{CH}_{2}$ \\
\hline & & & & $(13) 34.86 \mathrm{CH}_{2}$ & & & - & 34.69 & 34.69 & $\mathrm{CH}_{2}$ \\
\hline & & $35.40 \mathrm{CH}$ & & & & & - & 35.28 & 35.28 & $\mathrm{CH}$ or $\mathrm{CH}_{3}$ \\
\hline & & & & $(15) 36.89 \mathrm{CH}_{2}$ & & & - & 35.88 & 35.88 & $\mathrm{CH}_{2}$ \\
\hline & & & & & & & - & 36.14 & 36.14 & $\mathrm{CH}$ or $\mathrm{CH}_{3}$ \\
\hline & & & $(16) 36.59 \mathrm{CH}$ & & & & - & 36.35 & 36.35 & $\mathrm{CH}$ or $\mathrm{CH}_{3}$ \\
\hline & & & & & & & - & 37.18 & 37.18 & $\mathrm{CH}$ or $\mathrm{CH}_{3}$ \\
\hline & $\begin{array}{c}41.45 \\
-42.38\end{array} \quad \mathrm{CH}_{2}$ & & & & & & 41.67 & $41.67-42.67$ & $41.67-42.67$ & $\mathrm{CH}_{2}$ \\
\hline & & & & & $\begin{array}{r}(1) 42.76 \\
-44.10\end{array} \mathrm{CH}_{2}$ & 44.10 & - & $\begin{array}{c}42.94,43.31 \\
44.04\end{array}$ & $\begin{array}{l}43.31 \\
44.04\end{array}$ & $\mathrm{CH}_{2}$ \\
\hline & & & $(15) 49.81 \quad \mathrm{C}$ & & & & - & 48.87 & 48.87 & C \\
\hline & & & & & & & - & 55.92 & 55.92 & c \\
\hline & & & & & & & - & 56.47 & 56.47 & C \\
\hline & & & & & & & - & 57.99 & 57.99 & $\mathrm{CH}_{2}$ \\
\hline & $58.73 \quad \mathrm{CH}_{2}$ & & $\begin{array}{cc:c}(3,6,9) & & \\
58.73 & \mathrm{CH}_{2} & \end{array}$ & $\begin{array}{cc}(3,6,9) \\
58.73\end{array} \quad \mathrm{CH}_{2}$ & & & 58.44 & 58.41 & 58.41 & $\mathrm{CH}_{2}$ \\
\hline & & $107.80 \quad \mathrm{CH}_{2}$ & & & & & - & 107.79 & 107.79 & $\mathrm{CH}_{2}$ \\
\hline & & & & & & & - & - & 120.80 & $(\mathrm{CH} ?)$ \\
\hline & & & & & 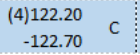 & 122.20 & - & $\begin{array}{c}122.19,122.37 \\
122.48\end{array}$ & $\begin{array}{c}122.19,122.37 \\
122.48\end{array}$ & C \\
\hline \multirow[t]{5}{*}{$123.49 \quad \mathrm{C}$} & & & & & & & 123.31 & 123.25 & 123.25 & C \\
\hline & & & & (18)122.89 C & & & - & 124.30 & 124.30 & C \\
\hline & & & & (16)128.75 C & & & - & 127.87 & 127.87 & C \\
\hline & & & & & $\begin{array}{r}(2) 130.28 \quad C \\
-132.02 \quad C\end{array}$ & 132.00 & - & $\begin{array}{l}130.27,131.13 \\
131.66,132.01\end{array}$ & $\begin{array}{l}130.27,131.13 \\
131.66,132.01\end{array}$ & $\mathrm{C}$ \\
\hline & & $151.20 \quad \mathrm{C}$ & & & & & - & - & - & \\
\hline
\end{tabular}




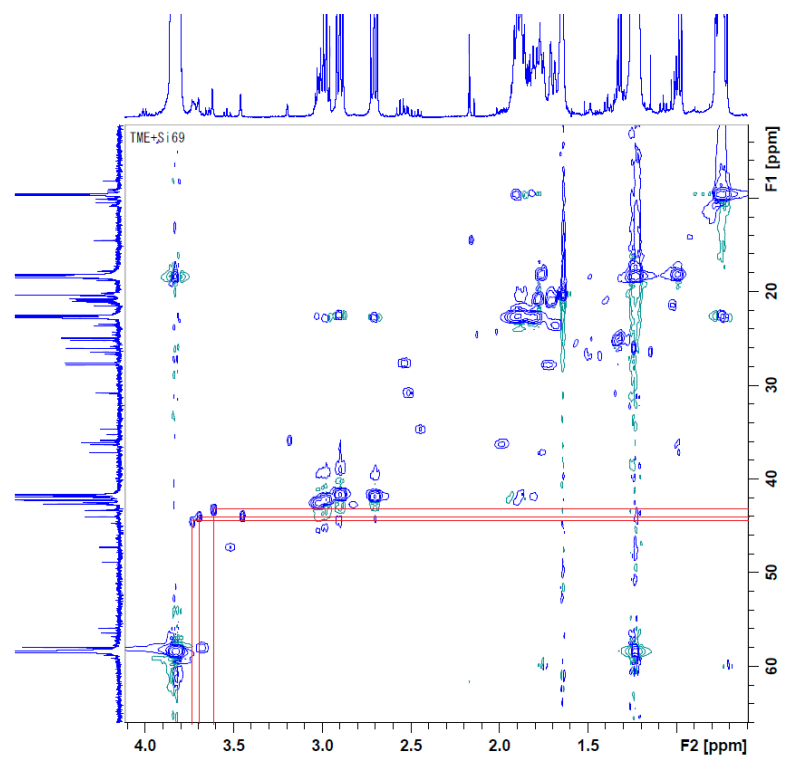

Figure 3-35: HSQC spectrum of TME / Si 69 mixture after heating at $170{ }^{\circ} \mathrm{C}$ for 5 hours

In the case of a sulfide silane, the chemical shift of the proton atom at the carbon atom next to the sulfur atom varies depending on the sulfur length as following; $S_{2}$ silane $=2.5$ $\mathrm{ppm}, \mathrm{S}_{3}$ silane $=2.9 \mathrm{ppm}, \mathrm{S}_{4}$ silane $=3.12 \mathrm{ppm}$, silanes of longer than $S_{5} \geq 3.17 \mathrm{ppm}$. In Figure 3-34, the decrease of longer sulfur chain silanes $\left(\geq S_{4}\right)$ together with the increase of $\mathrm{S}_{2}$ and $\mathrm{S}_{3}$ silanes by heating can be recognized. From the observations so far, it is reasonable to conclude that, as it has been proposed from the past, $[8,9,20,21]$ the active sulfur species which are released from the longer sulfur components in Si 69 by heating (sulfur donor effect of Si 69) can react with the allylic carbon in TME, which results in the generation of the cross-linked product $(\mathrm{A})$.

Several other new peaks, besides the peaks which correspond to the cross-linked product (A), have been observed in the ${ }^{13} \mathrm{C}$ NMR spectrum as summarized in Table 3-6. To clarify these further peaks, additional GC/MS measurements were carried out. As shown in Figure 3-36, the GC/MS spectrum of the sample after heating shows two new signals at 13.58 and $14.32 \mathrm{~min}$ with the mass value of 322 and $321 \mathrm{~m} / \mathrm{z}$, respectively. As it was confirmed in the Si 263 system, these masses seem to be those of the resulting reaction product between one mercapto-silane radical and one TME molecule. As a result of the consideration of the 
relationship between the actual chemical shifts and calculated values and also the observed correlation in COSY and HSQC measurements, the described products in Figure 3-37 seemed to be possible structures. Because of a very low peak intensity of the signals in the ${ }^{13} \mathrm{C} \mathrm{NMR}$ spectrum, a higher failure has to be considered. However, the generation of the proposed reaction products in Figure 3-37 based on the reaction between one TME molecule and one mercapto-silane component seems to have taken place most likely. Although it is not clear if the mercapto-silane radicals were generated by heating from the mercapto-silane component (Si 263) originally contained in Si 69 as an impurity or from the dissociation of $\mathrm{S}_{2}$ or longer components of $\mathrm{Si} 69$, it can be confirmed by this study that at least a mercaptosilane radical is present after heating of $\mathrm{Si} 69$ and reacts further with the olefin to a certain degree. The addition reaction products of $(\mathrm{EtO})_{3} \mathrm{Si}\left(\mathrm{CH}_{2}\right)_{3} \mathrm{~S}_{\mathrm{x}} \bullet$ radical, which can be produced by the dissociation of longer sulfur chain components of Si 69, to TME was not observed in any measurements. Therefore, it is reasonable to assume that a small amount of an addition reaction of the mercapto-silane radical to TME in combination with the sulfur cross-linking reaction is responsible for forming the chemical bound rubber in the Si 69 / silica filled compound at the $1^{\text {st }}$ mixing stage.

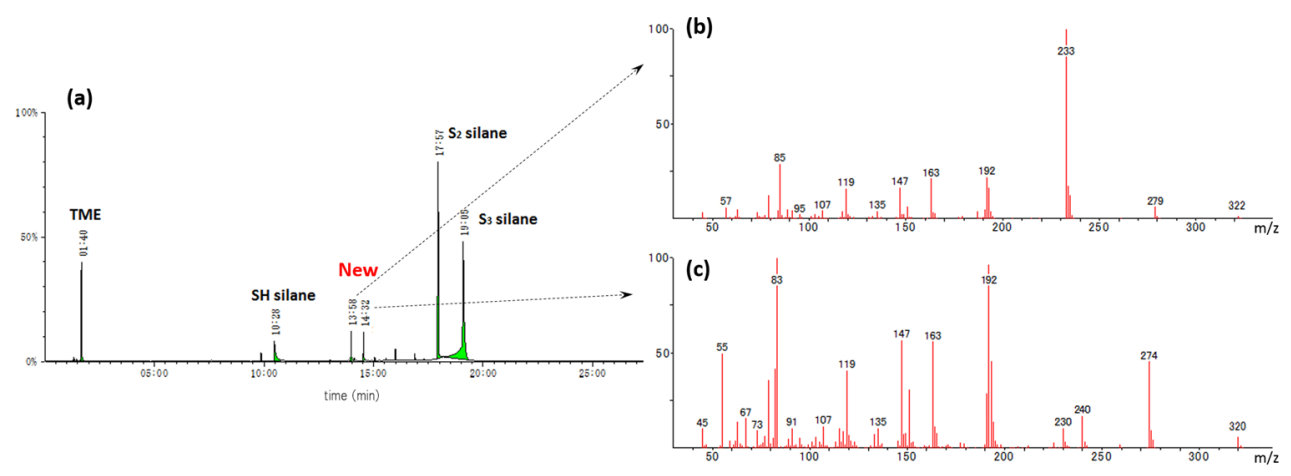

Figure 3-36: (a) GC/MS spectrum of TME / Si 69 mixture after heating at $170{ }^{\circ} \mathrm{C}$ for $60 \mathrm{~min}$, (b \& c) Mass spectrum of the new resulting peak at $13.58 \mathrm{~min}$ and $14.32 \mathrm{~min}$, respectively

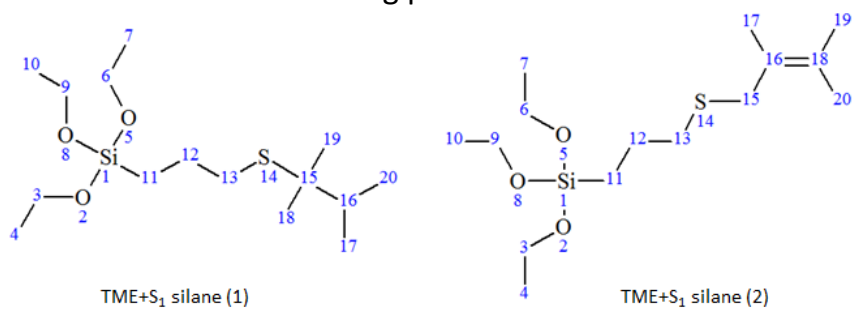

Figure 3-37: Possible reaction products between TME and Si 69 after heating at $170{ }^{\circ} \mathrm{C}$ for $60 \mathrm{~min}$ 


\section{3-3-3-2 3m1p / Si 69 system}

From all investigated olefin / Si 263 systems that one containing 3m1p led to the highest amount of addition product, as explained in the 3-3-2 section. Therefore, the generation of this addition product was checked in the Si 69 system, as well.

In the GC/MS measurement of the mixture of $3 \mathrm{~m} 1 \mathrm{p}$ and $\mathrm{Si} 69$ after heating at $170{ }^{\circ} \mathrm{C}$ for $60 \mathrm{~min}$, a new peak at $14.06 \mathrm{~min}$ is observed. Since this peak has almost the same retention time and also the same fragment pattern as the one observed in the $3 \mathrm{~m} 1 \mathrm{p} / \mathrm{Si} 263$ system (Figure 3-38), the formation of an addition reaction product of a mercapto-silane to the double bond in $3 \mathrm{~m} 1 \mathrm{p}$ can be confirmed in both systems. However, since this product cannot be observed in the ${ }^{13} \mathrm{C}$ NMR spectrum in the same sample, it can be judged that the produced amount is very small under the chosen conditions.

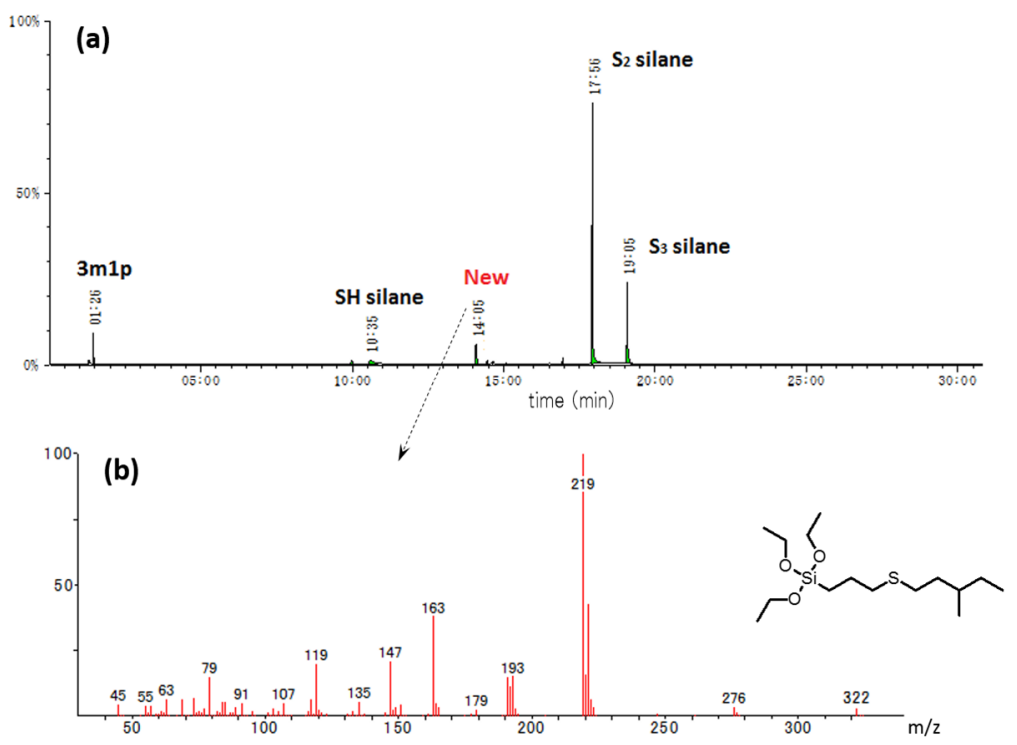

Figure 3-38: (a) GC/MS spectrum of 3m1p / Si 69 mixture after heating at $170^{\circ} \mathrm{C}$ for $60 \mathrm{~min}$, (b) Mass spectrum of the new resulting peak at $14.05 \mathrm{~min}$ 
By increasing the heating time up to three hours and five hours respectively, the corresponding peaks to this product could be identified, as shown in Figure 3-39.

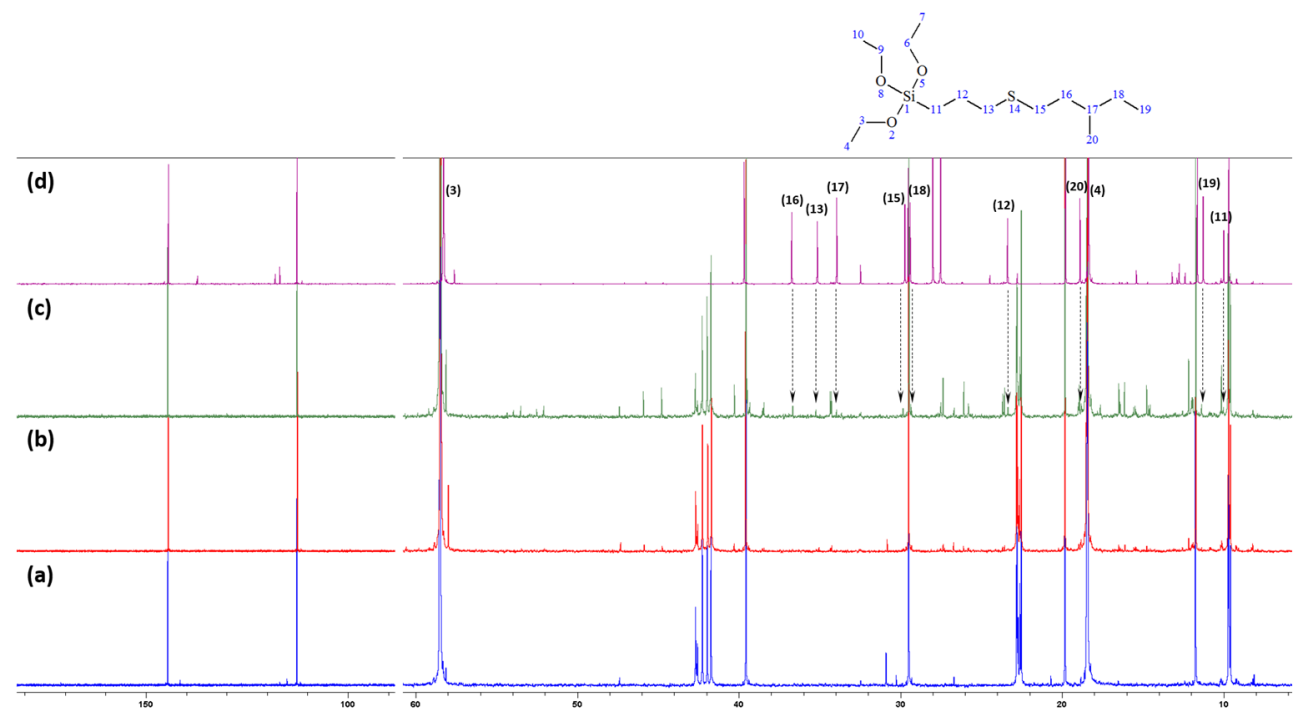

Figure 3-39: ${ }^{13} \mathrm{C}$ NMR spectra of (a) 3m1p / Si 69 mixture before heating, (b) after heating at $170^{\circ} \mathrm{C}$ for $60 \mathrm{~min},(\mathrm{c})$ after heating at $170^{\circ} \mathrm{C}$ for 5 hours and (d) $3 \mathrm{~m} 1 \mathrm{p} / \mathrm{Si} 263$ mixture after heating at $170{ }^{\circ} \mathrm{C}$ for $30 \mathrm{~min}$.

Several other new peaks, besides the peaks which correspond to the addition product, can be observed in the ${ }^{13} \mathrm{C}$ NMR spectrum of the mixture between $3 \mathrm{~m} 1 \mathrm{p}$ and Si 69 after heating, as shown in Figure 3-39 (b) and (c). The exact structure of the products which belongs to the new observed peaks was evaluated in the same way as for the TME system. In the case of the TME system, as the active sulfur species react in the allylic position, the resulting cross-linked product still has a double bond in the molecule. The chemical shift of this double bond appears at a shifted position from the original place of the TME double bond, therefore, it was possible to be observed in the NMR spectra. However, in the case of the $3 \mathrm{~m} 1 \mathrm{p} / \mathrm{Si} 69$ system, no new peaks within the chemical shift range of a double bond are observed neither in ${ }^{1} \mathrm{H}$ (Figure $3-40$ ) nor in ${ }^{13} \mathrm{C}$ NMR of the sample after heating. 
Therefore, it can be concluded that the same type of cross-linked products as detected in the TME system is not formed in the $3 \mathrm{~m} 1 \mathrm{p}$ system.

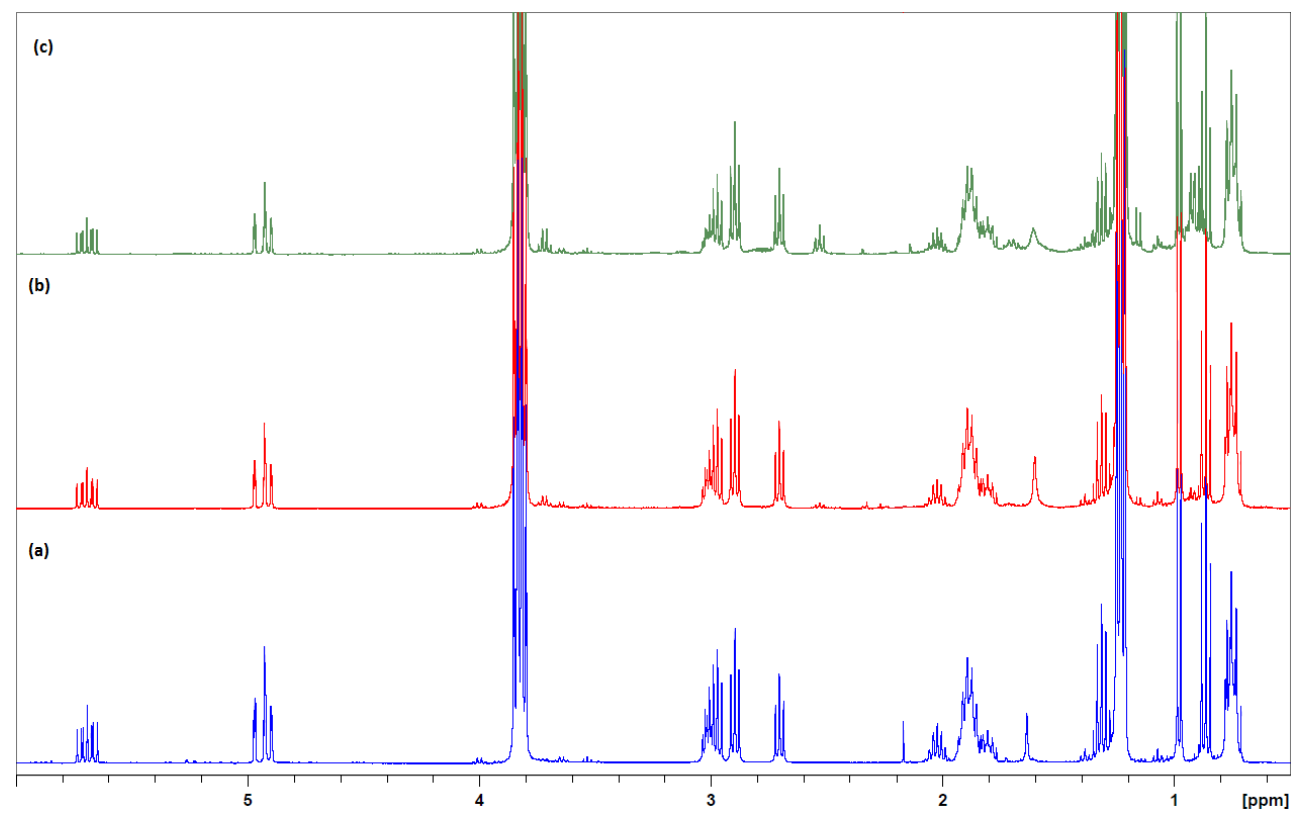

Figure 3-40: ${ }^{1} \mathrm{H}$ NMR spectra of 3m1p / Si 69 mixture (a) before heating, (b) after heating at $170{ }^{\circ} \mathrm{C}$ for $60 \mathrm{~min}$, (c) after heating at $170{ }^{\circ} \mathrm{C}$ for 5 hours

However, it can be observed that the total peak intensity of double bonds decreases after heating. Two options can be considered to explain this phenomena. The first one is that the active sulfur species from Si 69 in the $3 \mathrm{~m} 1 \mathrm{p}$ system have reacted with the double bond directly to produce a sulfur cross-linked product as shown in Figure 3-41, and the other one is the dissociated silane $\left((\mathrm{EtO})_{3} \mathrm{Si}\left(\mathrm{CH}_{2}\right)_{3} \mathrm{~S}_{\mathrm{x}}\right)$ components added the vinyl double bond of $3 \mathrm{~m} 1 \mathrm{p}$. The calculated chemical shift of the sulfur cross-linked product (Figure 3-41) does not fit with the actual signals, therefore, the generation of this product can be excluded.<smiles>CCC(C)CCSSCCC(C)CC</smiles>

Figure 3-41: Considered structure of the reaction product in the 3m1p / Si 69 system 
Figure 3-42 (a) and (b) show the ESI+ chromatogram in the LC/MS measurement of the sample before and after heating at $170{ }^{\circ} \mathrm{C}$ for 5 hours. By heating, several peaks such as those at 16.4, 19.0, 19.8 and $20.7 \mathrm{~min}$ become visible. Mass spectra of these peaks are shown in Figure 3-42 (c), (d), (e) and (f), respectively. The peak at $16.4 \mathrm{~min}$ shows $345 \mathrm{~m} / \mathrm{z}$ as a maximum value, which corresponds to the molecular weight of the ion adducts of the addition product between $3 \mathrm{~m} 1 \mathrm{p}$ and mercapto-silane with sodium $\left(\mathrm{Na}^{+}\right)\left[3 \mathrm{~m} 1 \mathrm{p}+\mathrm{Si} 263+\mathrm{Na}^{+}\right]$. It is known from literature that when methanol is used as a mobile phase in LC/MS measurements in combination with the ESI ionization method, sodium ion adduct is often detected.[29] The peaks at 19.0, 19.8 and $20.7 \mathrm{~min}$ have 341,373 and $405 \mathrm{~m} / \mathrm{z}$ as maximum values, respectively, which increases by $32 \mathrm{~m} / \mathrm{z}$. The value of this $32 \mathrm{~m} / \mathrm{z}$ fits with the atomic mass of sulfur. Therefore, these peaks seem to correspond to the addition reaction products of (EtO) $)_{3} \mathrm{Si}\left(\mathrm{CH}_{2}\right)_{3} \mathrm{~S}_{3}$ to 5 components with $3 \mathrm{~m} 1 \mathrm{p}$ which have lost one ethoxy group, as shown in Figure 3-42 (on the right side). Whether the addition reactions are via a Markovnikov addition or an Anti-Markovnikov addition cannot be judged only by the information of mass values. But, it seems to be that by increasing heating time, the addition reaction of the dissociated silane components having different sulfur length to a $3 \mathrm{~m} 1 \mathrm{p}$ molecule is possible to occur.
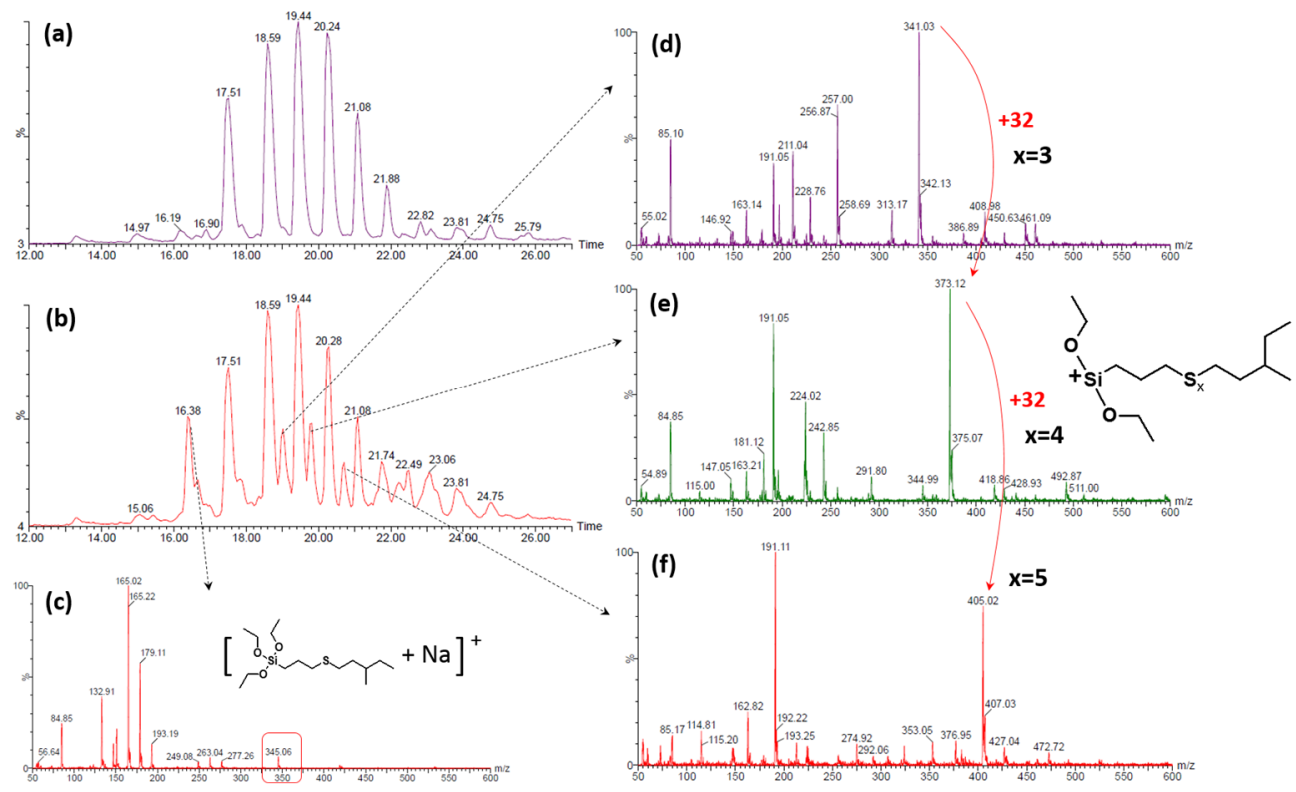

Figure 3-42: ESI+ chromatogram in the LC/MS measurement 3m1p / Si 69 mixture (a) before heating, (b) after heating at $170^{\circ} \mathrm{C}$ for 5 hours, (c) - (f) Mass spectra at 16.4, 19.0, 19.8 and $20.7 \mathrm{~min}$ 
However, these peaks of adducts are not clearly observed in the sample after heating at $170{ }^{\circ} \mathrm{C}$ for $60 \mathrm{~min}$, thus the concentration of them seems to be very low. Considering additionally the fact that no sulfur cross-linked products were detected unlike the TME / Si 69 system, it can be concluded that the presence of the vinyl structure does also not contribute to increase-the linking points between olefin and silane in the case of the Si 69 system. This role of the vinyl structure is very different from that in the Si 263 system.

\section{3-3-3-3 C3H / Si 69 system}

In the $\mathrm{C} 3 \mathrm{H}$ system, not only addition reaction products which have been reported in the literature,[10] but also isomerization products as observed in the Si 263 system (Chapter 33-2-2) can be expected as resulting structures after heating. Therefore, decane was used as an internal standard to calculate the concentration of each component before and after heating.

The ${ }^{13} \mathrm{C}$ NMR spectra of the $\mathrm{C} 3 \mathrm{H} / \mathrm{Si} 69$ system before and after heating at $170{ }^{\circ} \mathrm{C}$ for 30 min are shown in Figure 3-43. Isomerization of $\mathrm{C} 3 \mathrm{H}$ was indeed observed after heating in the Si 69 system as seen in the same figure. However, on one hand three isomers including a small generation of trans-2-hexene and cis-2-hexene were observed in the Si 263 system, on the other hand only one isomer, trans-3-hexene, is now observed in the Si 69 system. Figure 3-44(a) shows the change of the concentration ratio of each isomer as a function of heating time at different temperatures. For comparison, the results in the Si 263 system are shown again in Figure 3-44 (b). When the rate of the decrease of the cis-3 structure which is the original structure of the olefin is named as the isomerization rate, the rate in the $\mathrm{Si}$ 263 system is significantly higher than that in the Si 69 system, especially at temperatures higher than $150{ }^{\circ} \mathrm{C}$. The isomerization mechanism seems to be via the reversible addition reaction of radical components and/or persulfenylthiol components or the allylic hydrogen abstraction. Therefore, it can be concluded that the abilities of the addition reaction as well as the allylic hydrogen abstraction of Si 69 to cis-3-hexene are much lower compared to those in the Si 263 system, which is the same tendency observed already in the TME and $3 \mathrm{~m} 1 \mathrm{p}$ systems. 


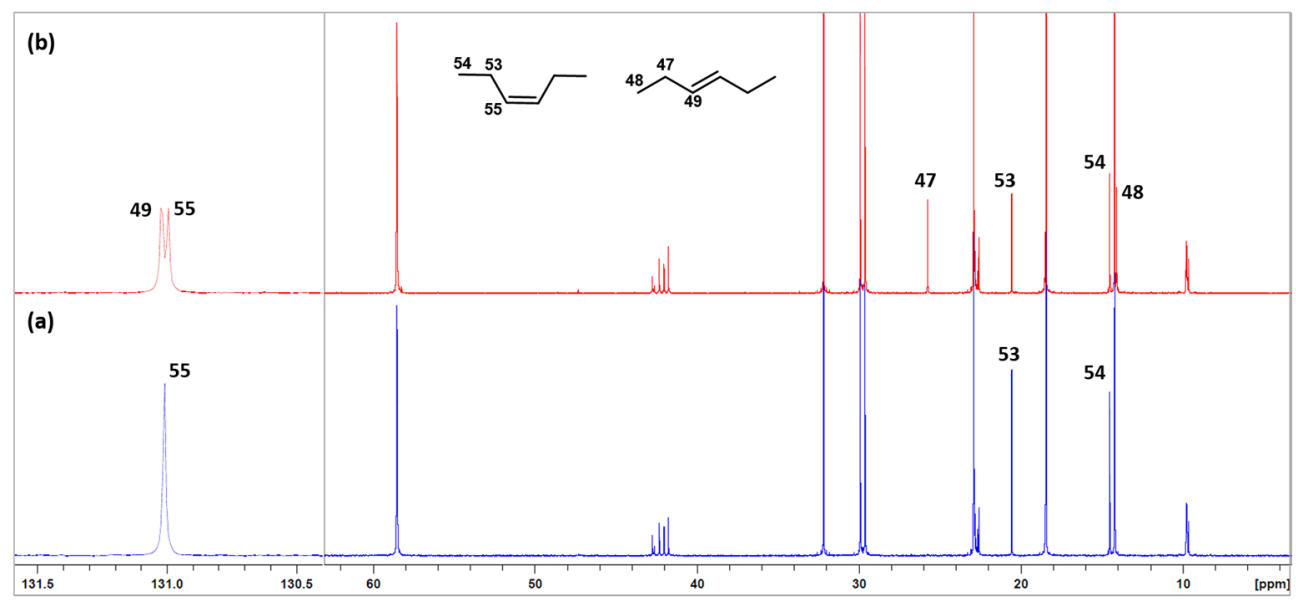

Figure 3-43: ${ }^{13} \mathrm{C}$ NMR spectra of $\mathrm{C} 3 \mathrm{H} /$ Si 69 mixture (a) before heating and (b) after heating at $170{ }^{\circ} \mathrm{C}$ for $30 \mathrm{~min}$.
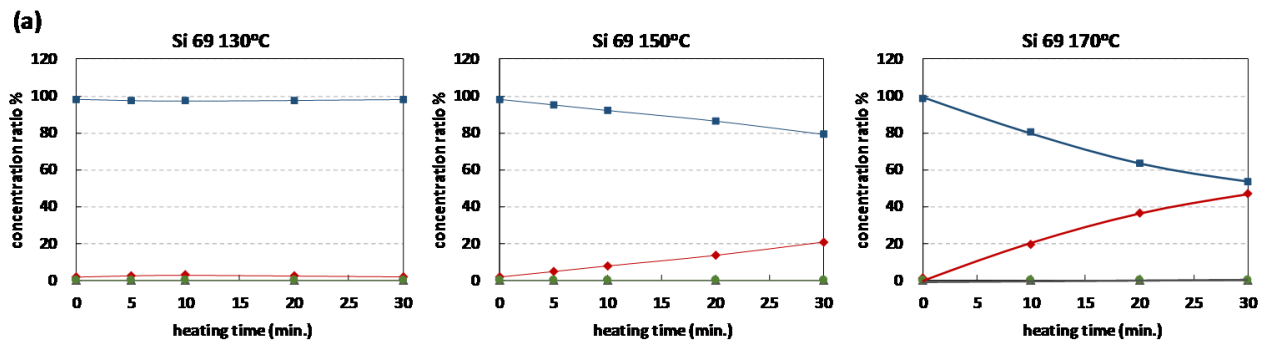

- cis-3-hexene trans-3-hexene $\Delta$ trans-2-hexene

cis-2-hexene
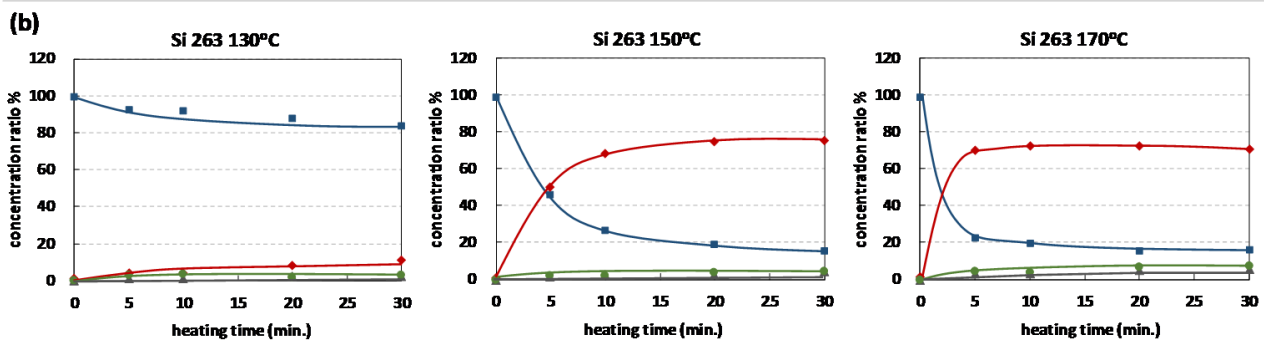

a cis-3-hexene trans-3-hexene $\Delta$ trans-2-hexene cis-2-hexene

Figure 3-44: Change of concentration ratio of each olefin component (a) in C3H / Si 69 system and (b) in $\mathrm{C} 3 \mathrm{H} / \mathrm{Si} 263$ system 
Regarding addition reaction products, the same structure as observed in the Si 263 system where $\mathrm{S}_{1}$ silane component added at a double bond of $\mathrm{C} 3 \mathrm{H}$, was able to be detected in the sample after heating at $170{ }^{\circ} \mathrm{C}$ for 30 minutes, though the amount was much smaller than that inside the Si 263 system. However, the addition reaction structures where dissociated silanes have coupled to a $\mathrm{C} 3 \mathrm{H}$ molecule via more than two sulfur atoms, which were reported in the literature were not detected in this study.[10] The clear reason for this is unknown, but if the addition reaction is a reversible reaction, it seems reasonable that $\mathrm{T} 3 \mathrm{H}$ remains as a main component from the stability of the molecules' point of view. In this case, it is not important if the addition reaction is of an Anti-Markovnikov type by a persulfenyl radical or of a Markovnikov type by a persulfenyl thiol.

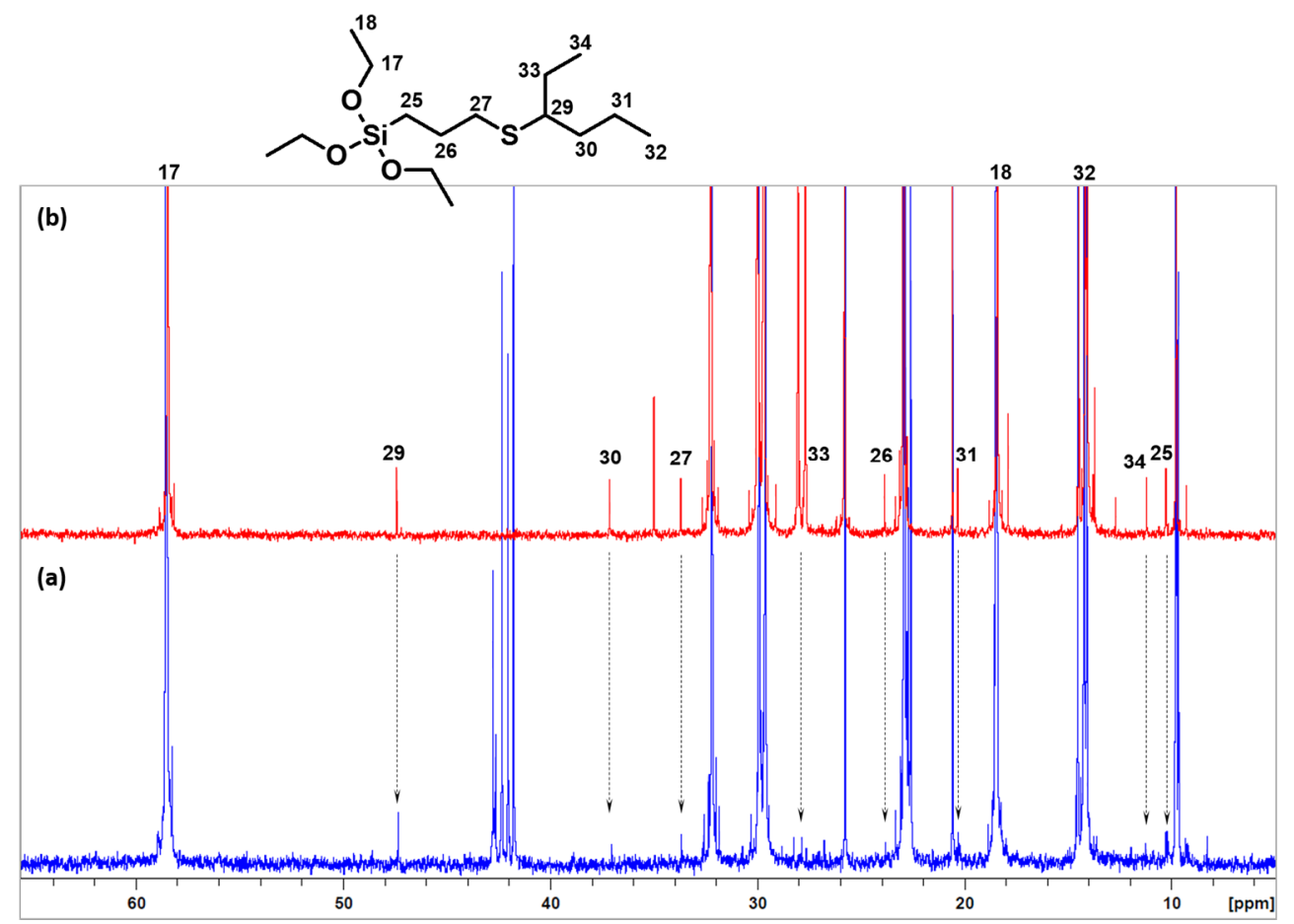

Figure 3-45: ${ }^{13} \mathrm{C}$ NMR spectra of (a) $\mathrm{C} 3 \mathrm{H} /$ Si 69 mixture after heating at $170{ }^{\circ} \mathrm{C}$ for $30 \mathrm{~min}$ and (b) $\mathrm{C} 3 \mathrm{H} / \mathrm{Si} 263$ mixture after heating at $170{ }^{\circ} \mathrm{C}$ for $30 \mathrm{~min}$

In summary, the isomerization rate in the $\mathrm{C} 3 \mathrm{H} / \mathrm{Si} 69$ system was lower and the amount of the detected isomers was smaller compared to that one in the $\mathrm{C} 3 \mathrm{H} / \mathrm{Si} 263$ system. Therefore, it can be concluded that Si 69 has a lower ability of an addition reaction and 
allylic hydrogen abstraction, which result in a lower generation of radical species from olefins, than Si 263.

\section{3-3-3-4 T3M3H / Si 69 system}

The influence of a methyl group at a double bond on the reactivity between the olefin and Si 69 was investigated. In this system, similar to that what was already observed in the Si 263 system, the isomerization of T3M3H takes also place and the direct reaction product between $\mathrm{T} 3 \mathrm{M} 3 \mathrm{H}$ and silane cannot be detected. The isomerization rate in this system is the same as in the $\mathrm{C} 3 \mathrm{H}$ system which means that it is significantly lower than that in the $\mathrm{Si} 263$ system as shown in Figure 3-46. The number of observed isomers is also lower in the T3M3H / Si 69 system than that in the ТЗМЗH / Si 263 system. This trend is similar to that one in both $\mathrm{C} 3 \mathrm{H}$ systems.

(a)
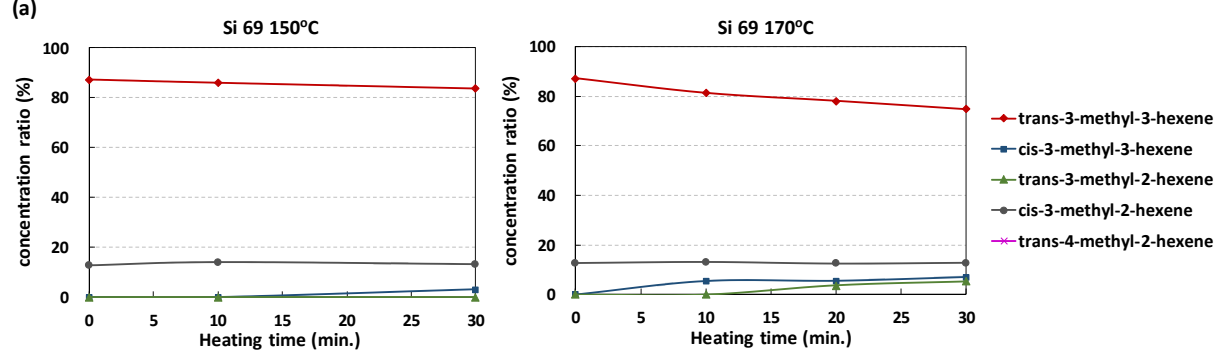

(b)
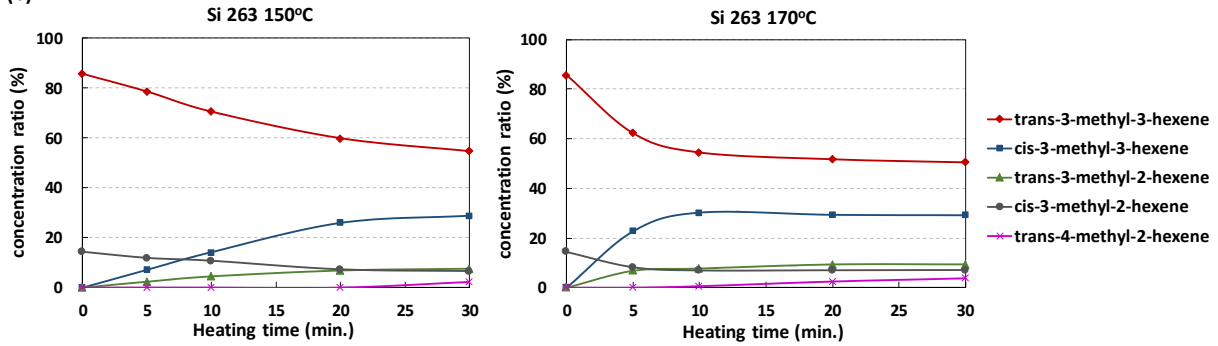

Figure 3-46: Change of the concentration ratio of each olefin component (a) in $\mathrm{T3M} 3 \mathrm{H} / \mathrm{Si}$ 69 system and (b) in T3M3H / Si 263 system 


\section{3-3-3-5 Summary in Si 69 systems}

Using four different olefins and Si 69, the reaction possibility, the mechanism and the resulting structures were investigated. On the one hand, in the case of TME and $3 \mathrm{~m} 1 \mathrm{p}$ systems, where changes in the ${ }^{1} \mathrm{H}$ and ${ }^{13} \mathrm{C}$ NMR spectra by heating at $170{ }^{\circ} \mathrm{C}$ for 30 minutes were too small to identify the resulting structures, therefore, the resulting reaction structures were investigated by increasing heating time up to 1, 3 and 5 hours. On the other hand, a certain level of changes were observed in the NMR spectra after heating at $170{ }^{\circ} \mathrm{C}$ for 30 minutes in the $\mathrm{C} 3 \mathrm{H}$ and $\mathrm{T} 3 \mathrm{M} 3 \mathrm{H}$ systems. And since they were mainly isomers, the isomerization rate was compared with that in the Si 263 system using decane as an internal standard.

Inside the Si 69 system similar phenomena were observed as in the Si 263 system. Due to the fact that there is a certain possibility that during the heating of Si 69 a small amount of mercapto-silane radicals are formed, the addition reaction product of the mercapto-silane fragment and the olefin was detected in the TME, $3 \mathrm{~m} 1 \mathrm{p}$ and $\mathrm{C} 3 \mathrm{H}$ systems. However, the amount inside the Si 69 system was significantly smaller than in the Si 263 system. Since it was very low even in the $3 \mathrm{~m} 1 \mathrm{p} / \mathrm{Si} 69$ system compared to the largest amount observed in the Si 263 system, it was indicated that the amount of the $S_{1}$ radical which can be produced by heating in the Si 69 system was considerably low.

Direct addition reaction products of an olefin with silane fragments, which have a sulfur length higher than two, were observed only to a very low amount in the $3 \mathrm{~m} 1 \mathrm{p} / \mathrm{Si} 69$ system after heating at $170{ }^{\circ} \mathrm{C}$ for longer than 60 minutes. However, those were not detected in the other olefin / Si 69 systems. Therefore, it can be concluded that those are the not main reaction products after heating only an olefin and Si 69 together. Furthermore, based on the results so far, it can be expected for the behavior in rubber that the higher vinyl content in the polymer blends does not contribute to increase the chemical bound rubber content in the Si 69 / silica filled compounds.

The cross-linked products where two mol of olefins are combined by sulfur coupled at the allylic carbons were observed in the TME system, same as already proposed in the past. $[8,9,20,21]$ The sulfur components seems to be released from Si 69 having more than two sulfur atoms. However, this kind of cross-linked products were not observed in the $3 \mathrm{~m} 1 \mathrm{p}, \mathrm{C} 3 \mathrm{H}$ or $\mathrm{T} 3 \mathrm{M} 3 \mathrm{H}$ systems. 
Similar to the Si 263 system, an isomerization was observed in the $\mathrm{C} 3 \mathrm{H}$ and T3M3H system. However, the isomerization rate was slower and the number of isomers was lower than those in the Si 263 systems. This indicates that the ability of the addition reaction as well as the allylic hydrogen abstraction from double bonds are much lower inside the Si 69 system compared to those in the Si 263 system. Therefore, it can be assumed that Si 69 has a lower ability to create radical species from olefins compared to Si 263.

Furthermore, a decrease of the double bond after heating was observed in the $\mathrm{C} 3 \mathrm{H}$ and T3M3H / Si 69 systems which does not occur in the Si 263 system. Taking additionally into consideration that the addition reaction products of Si 69 fragments having more than two sulfur atoms were not detected, this seems to be because of sulfur species released from $\mathrm{Si}$ 69. The resulting structure might be a sulfur pendant structure.

Overall, the reaction mechanisms inside the Si 69 system are significantly different from those of the Si 263 system. 


\section{3-4 References}

[1] O. Klockmann, P. Albert, A. Hasse, K. Korth, Rubber World, (2006) 36-40

[2] O. Klockmann, A. Hasse, German Rubber Conference 2006, Nuremberg, July 3-6 (2006)

[3] O. Klockmann, J. Hahn, H. Scherer, International Rubber Conference 2009, Nuremberg, June 29-July 2 (2009)

[4] O. Klockmann, A. Blume, A. Hasse, 172nd Am. Chem. Soc., Rubber Div. Meeting, Cleveland, 87, October 16-18 (2007)

[5] O. Klockmann, 174th Am. Chem. Soc., Rubber Div. Meeting, Louisville, Kentucky, October 14-16 (2008)

[6] M. York, Tire Tech 2015, Cologne, February 10-12 (2015)

[7] A. Blume, Kautsch. Gummi Kunstst., 64 (2011) 38-43

[8] S.C. Debnath, R.N. Data, J.W.M. Noordermeer, Rubber Chem. Technol., 76 (2003) 13111328

[9] U. Görl, J. Munzenberg, H.D. Luginsland, A. Muller, Kautsch. Gummi Kunstst., 52 (1999) 588-598

[10] H. Hayen, M.M. Alvarez-Grima, S.C. Debnath, J.W.M. Noordermeer, U. Karst, Anal. Chem., 76 (2004) 1063-1068

[11] R. J. Abraham, J. Fisher, P. Loftus, Introduction to NMR spectroscopy, Wiley, Chichester (1988) 271

[12] P. Mandal, S. Ponnupandian, S. Choudhury, N. Singha, Rubber Chem. Technol., 90 (2017) 550-561

[13] D. Lenko, S. Schlögl, A. Temel, R. Schaller, A. Holzner, W. Kern, J. Appl. Polym. Sci., 129 (2013) 2735-2743

[14] O. Ito, Journal of the Society of Rubber Science and Technology, Japan, 61 (1988) 201208

[15] M. Takebayashi, Journal of Synthetic Organic Chemistry, Japan, 20 (1962) 218-231

[16] O. Shigeru, Journal of Synthetic Organic Chemistry, Japan, 26 (1968) 327-341

[17] C. E. Hoyle, T. Y. Lee, T. J. Roper, Polym. Sci. Part A: Polym. Chem., 42 (2004) 5301-5338

[18] U. Biermann, W. Butte, R. Koch, P.A. Fokou, O. Turunc, M.A.R. Meier, J.O. Metzger, Chem. Eur. J., 18 (2012) 8201-8207

[19] P. Versloot, J.G. Haasnoot, J. Reedijk, M. Van Duin, J. Put, Rubber Chem. Technol., 70 (1997) 106-119 
[20] S. Mihara, "Reactive processing of silica-reinforced tire rubber: new insight into the time and temperature-dependence of silica rubber interaction", PhD. Thesis: 2009, Dept. of Elastomer Technology and Engineering, Univ. of Twente, Enschede, the Netherlands.

[21] L.A.E.M Reuvekamp, "Reactive mixing of silica and rubber for tires and engine mounts", Thesis: 2003, Dept. Rubber Technol., Univ. of Twente, Enschede, the Netherlands

[22] Wisut Kaewsakul, "Silica-Reinforced Natural Rubber for Low Rolling Resistance, EnergySaving Tires", Thesis: 2013, Dept. Rubber Technol., Univ. of Twente: Enschede, the Netherlands

[23] M. R. Kresja, J. L. Koenig, Rubber Chem. Technol., 66 (1993) 376-410

[24] R.S. Clough, J.L. Koenig, Rubber Chem. Technol., 62 (1989) 908-927

[25] A.M. Zaper, J.L. Koenig, Rubber Chem. Technol., 60 (1987) 252-277

[26] M.M. Coleman, J.R. Shelton, J.K. Koenig, Ind. Eng. Chem., Prod. Res. Develop., 13 (1974) 154-166

[27] J.R. Wolfe, T.L. Pugh, A.S. Killian, Rubber Chem. Technol., 41 (1968) 1329-1338

[28] C.G. Moore, M. Porter, J. Chem. Soc., (1965) 6390-6403

[29] http://www.jsac.or.jp/bunseki/pdf/bunseki2007/200707nyumon.PDF, Accessed day: 05/12/2017 


\section{Chapter 4}

\section{Model compound study-2: \\ Influence of silica, $\mathrm{ZnO}, \mathrm{CBS}$ and sulfur on the reaction between mercapto-silane and model olefin}

\section{4-1 Introduction}

Rubber compounds for tire treads are a mixture of various ingredients. Sometimes, the total number of them can be even more than ten species. Many of them are needed to have different chemical reactions during the tire production stages or the tire usage period to be able to display the expected effect. In many cases more than two ingredients are involved in the reaction scheme, which makes the mechanism complicated and difficult to elucidate. One typical example for it is the vulcanization reaction. Despite the fact that it has been used for such a long time, the complex reaction mechanism and the exact structure of intermediates have not been clarified completely.

The silica - silane technology has become standard in the tire industry nowadays, but the introduction of the silane coupling agent has brought further complexity in terms of chemical reactions inside the system. The silane coupling agent can react not only with the silica surface (silanization reaction) but as well as with the rubber, and furthermore, there is also the possibility that it reacts or at least interacts with other ingredients. Therefore, the number of possible reactions during production stages has significantly increased. For example, although an amine type of secondary accelerator such as DPG is normally used in combination with a sulfenamide type of primary accelerator such as CBS in silica filled compounds, DPG was found to be able to accelerate the silanization reaction as well. However, DPG has become suspect for its toxicity since it contains an aromatic amine which can release highly toxic free aniline during mixing and vulcanization. Therefore, there is a new task for the researchers to find an alternative amine which has a similar catalytical effect on the silanization reaction.[1-3] The direct reaction possibility between DPG and the silane (TESPT) was also implied, which would result in a higher consumption of DPG during mixing.[1] Another ingredient which might influence the silanization reaction is $\mathrm{ZnO}$, 
normally used to boost as an activator the effectivness of organic accelerators. It has been reported that, on one hand $\mathrm{ZnO}$ and / or $\mathrm{Zn}^{2+}$ can interact with the silica surface resulting in a slowing down of the silanization reaction rate, on the other hand $\mathrm{ZnO}$ doesn't affect the reaction rate between an olefin and sulfur.[4]

Considering the above mentioned points, it is significantly necessary to know the ingredients which might affect the mercapto-silane / polymer reaction. Therefore, in this chapter, the influence of silica, $\mathrm{ZnO}, \mathrm{CBS}$ and sulfur on the reaction between mercaptosilane and a model olefin will be investigated. The different reaction mechanisms of mercapto-silane with polymer compared to that of sulfide-silane with polymer were already indicated to be responsible for the characteristics of mercapto-silane compounds in Chapter 3. Therefore, to identify all influencing parameters and ingredients which affect the mercapto-silane / polymer reaction will lead to a better reaction control.

\section{4-2 Experimental}

\section{4-2-1 Sample preparation}

The used materials including their molecular weight of this study are summarized in Table 4-1.

Table 4-1: Material list for the model olefin study

\begin{tabular}{ccccc}
\hline Material & Abbreviations & Source & $\begin{array}{c}\text { Molecular weight } \\
(\mathrm{g} / \mathrm{mol})\end{array}$ & $\begin{array}{c}\text { Specific gravity } \\
\left(\mathrm{g} / \mathrm{cm}^{3}\right)\end{array}$ \\
\hline 3-methyl-1-pentene & $3 \mathrm{~m} 1 \mathrm{p}$ & Sigma-Aldrich & 84.16 & 0.67 \\
(3-Mercaptopropyl)triethoxysilane & Si 263 & Evonik & 238.42 & 0.99 \\
ULTRASIL 7005 & silica & Evonik & $\left(\right.$ CTAB $\left.164 \mathrm{~m}^{2} / \mathrm{g}\right)$ & 2.00 \\
Zinc oxide & ZnO & Merck & 81.41 & 5.61 \\
N-Cyclohexyl-2-benzothiazole sulfenamide & CBS & Flexsys & 264.42 & 1.31 \\
sulfur & & Sigma Aldrich & 256.52 & 2.07 \\
Decane & & Sigma Aldrich & 142.28 & 0.73 \\
\hline
\end{tabular}

As a pre-treatment of the ingredients, Si 263 and decane were flushed with nitrogen, respectively. Olefins were used without the procedure considering the relatively lower boiling point. For the calculation of the required amount of silane in the silica system, the silanol concentration at the silica surface which are able to be involved in the silanization reaction is estimated as $2.5 \mathrm{OH} / \mathrm{nm}^{2}$.[5] Based on this assumption, the concentration of $3 \mathrm{~m} 1 \mathrm{p}$ and Si 263 inside the solution was adjusted to an isomolar content. For the 
experiments in the systems with $\mathrm{ZnO}, \mathrm{CBS}$ and/or sulfur, one fourth mole of them relative to $3 \mathrm{~m} 1 \mathrm{p}$ or silane were added to the mixture. The composition of all investigated systems is shown in Table 4-2. The samples were prepared in glass vials $(20 \mathrm{ml})$, and decane was added as a solvent for all samples. The reaction was carried out by placing the vials in the rotation device shown in Figure 4-1. Each vial was heated up to the desired specific temperature for predetermined durations. After the reaction, the vials were cooled down immediately in an ice bath to stop the reaction. The reaction mixture was filtered by using a $45 \mu \mathrm{m}$ porous filter and prepared for the GC measurement.

Figure 4-1: Rotation and heating machine

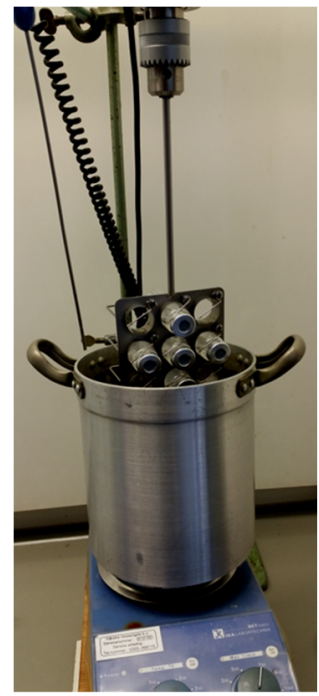

Table 4-2: Composition of all investigated systems

\begin{tabular}{cccccccccc}
\hline Material & Mix 1 & Mix 2 & Mix 3 & Mix 4 & Mix 5 & Mix 6 & Mix 7 & Mix 8 & Mix 9 \\
\hline 3m1p & $0.0855 \mathrm{ml}$ & - & $0.500 \mathrm{ml}$ & - & $0.500 \mathrm{ml}$ & - & $0.500 \mathrm{ml}$ & $0.500 \mathrm{ml}$ & $0.500 \mathrm{ml}$ \\
TME & - & - & - & $0.500 \mathrm{ml}$ & & & & & \\
\hline Si 263 & $0.164 \mathrm{ml}$ & $0.164 \mathrm{ml}$ & $0.962 \mathrm{ml}$ & $0.962 \mathrm{ml}$ & $0.962 \mathrm{ml}$ & $0.962 \mathrm{ml}$ & $0.962 \mathrm{ml}$ & $0.962 \mathrm{ml}$ & $0.962 \mathrm{ml}$ \\
\hline silica & $1.000 \mathrm{~g}$ & $1.000 \mathrm{~g}$ & - & - & - & - & - & - & - \\
ZnO & - & - & - & - & $0.0811 \mathrm{~g}$ & $0.0811 \mathrm{~g}$ & $0.0811 \mathrm{~g}$ & - & - \\
CBS & - & - & - & - & - & - & - & $0.263 \mathrm{~g}$ & - \\
sulfur & - & - & - & - & - & - & $0.255 \mathrm{~g}$ & - & $0.255 \mathrm{~g}$ \\
\hline Decane & $9.000 \mathrm{ml}$ & $9.0855 \mathrm{ml}$ & $9.000 \mathrm{ml}$ & $9.000 \mathrm{ml}$ & $9.000 \mathrm{ml}$ & $9.500 \mathrm{ml}$ & $9.000 \mathrm{ml}$ & $9.000 \mathrm{ml}$ & $9.000 \mathrm{ml}$ \\
\hline
\end{tabular}

\section{4-2-2 Analytical method}

The Gas Chromatography (GC) measurement was carried out following the same settings as shown in Chapter 3.

\section{4-3 Results and Discussion}

\section{4-3-1 Influence of the presence or absence of olefin on the hydrophobation effect for silica}

In the previous chapter, the possibility that Si 263 reacts directly with $3 \mathrm{~m} 1 \mathrm{p}$, which has a vinyl double bond, was confirmed. In order to investigate if this addition reaction can occur 
also in the presence of silica, where a silane can also have a silanization reaction with the silica surface, experiments using additionally silica were carried out.

In the 3m1p / silica / Si 263 system, the following three possible reactions shown in Figure 4-2 can be considered. Only the silica / Si 263 reaction (silanization reaction) has occurred in the first case, and only the $3 \mathrm{~m} 1 \mathrm{p} / \mathrm{Si} 263$ reaction in the second case. The third case shows that the Si 263 which is coupled to the silica has coupled further to the $3 \mathrm{~m} 1 \mathrm{p}$. In order to clarify which reaction mechanism has finally occurred the reaction between silica and Si 263 (in absence of the olefin) was investigated additionally.

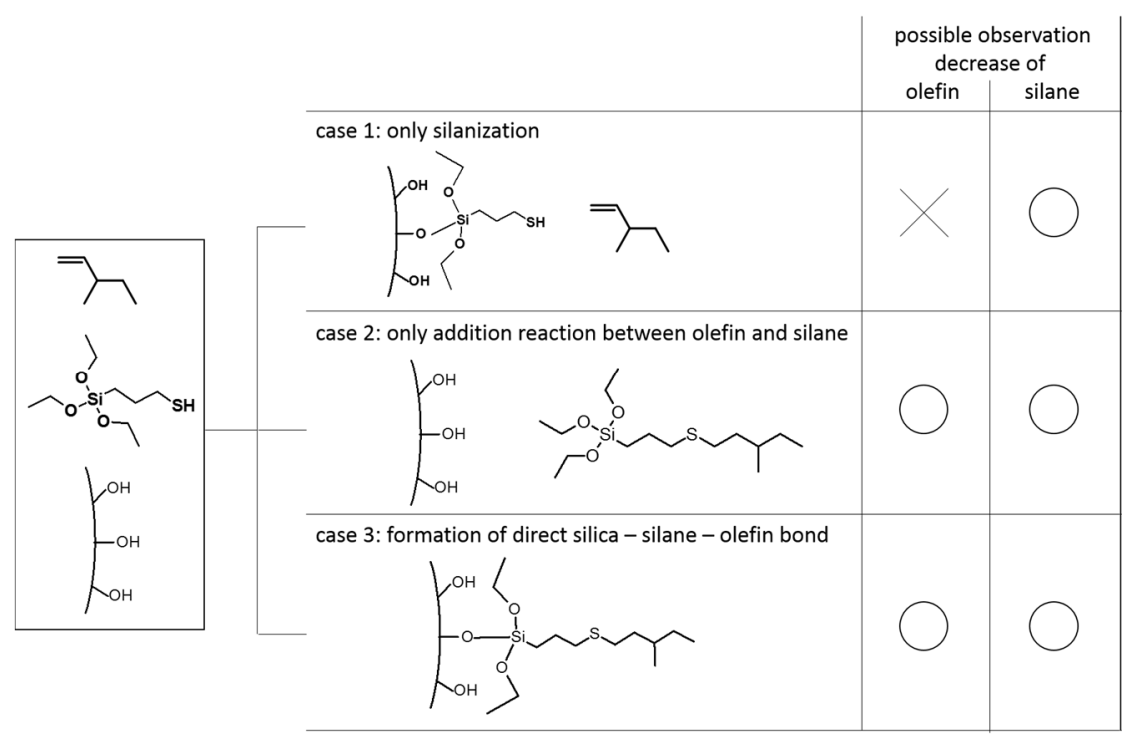

Figure 4-2: Possible reaction patterns in the 3m1p / silica / Si 263 system (o represents the decrease of the component, $\times$ represents no concentration change of the component.)

Figure 4-3 shows the peak area in the GC chromatogram of the remaining amounts of $3 \mathrm{~m} 1 \mathrm{p}$ and Si 263 in the $3 \mathrm{~m} 1 \mathrm{p}$ / silica / Si 263 sample or the silica / Si 263 sample after heating at $150{ }^{\circ} \mathrm{C}$ for specific times. Firstly, in the silica / Si 263 sample, a reduction of the Si 263 concentration with increasing heating time is observed $(22 \%$ after heating for 60 minutes at $150^{\circ} \mathrm{C}$ ), which indicates that the silanization reaction has occurred. Secondly, a decrease of the $3 \mathrm{~m} 1 \mathrm{p}$ concentration ( $8 \%$ after heating for 60 minutes at $150{ }^{\circ} \mathrm{C}$ ) is visible in the sample with $3 \mathrm{~m} 1 \mathrm{p}$. Considering the results in Chapter 3 , it is implied that some parts of $\mathrm{Si}$ 263 have reacted with $3 \mathrm{~m} 1 \mathrm{p}$ also in the presence of silica. Furthermore, as the $28 \%$ 
reduction of the Si 263 concentration is observed after heating for 60 minutes in this system, it can be concluded that about $20 \%$ (the difference between the above mentioned $28 \%$ and $8 \%$ ) of silane is used only for the silanization reaction.

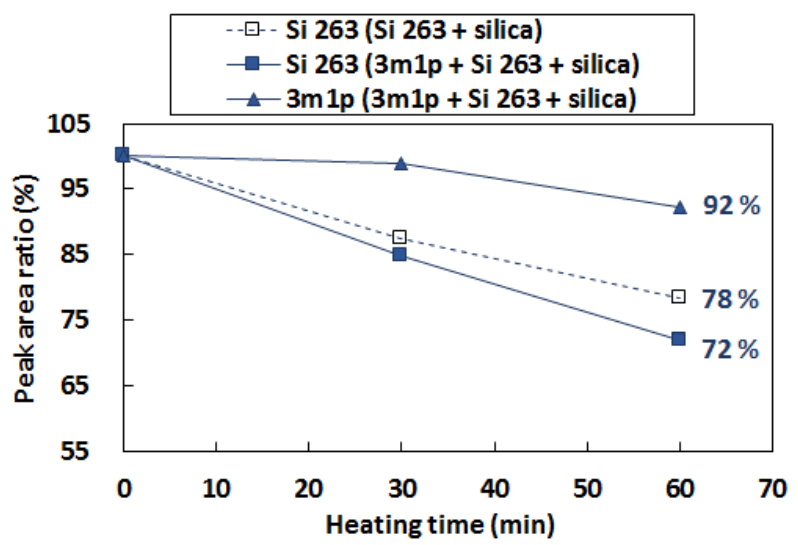

Figure 4-3: Change of the $3 \mathrm{~m} 1 \mathrm{p}$ and Si 263 concentration with heating time in the sample with or without $3 \mathrm{~m} 1 \mathrm{p}$

Knowing this, the next open question is if the reaction in the silica / 3m1p / Si 263 system follows case 2 or 3 which means whether the direct 3m1p - silica - Si 263 bonds have been formed or only the reaction between $3 \mathrm{~m} 1 \mathrm{p}$ and Si 263 has occurred. The indirect but clear answer to this question can be found in the GC chromatogram. It was confirmed in Chapter 3 that the peak of the addition reaction product between $3 \mathrm{~m} 1 \mathrm{p}$ and Si 263 is detectable in the chromatogram, but the peak is not visible in the system which contains additionally silica despite observing the reduction of $3 \mathrm{~m} 1 \mathrm{p}$. This can be interpreted as following that Si 263 which reacted with $3 \mathrm{~m} 1 \mathrm{p}$ also couples to silica and thus not remain in the solution, therefore, the addition reaction product itself is not detectable in the GC chromatogram which is taken from the solution.

As mentioned before, the reaction degree only between silica and Si 263 in the system with and without $3 \mathrm{~m} 1 \mathrm{p}$ is similar, which is $22 \%$ and $20 \%$, respectively. However, if the Si 263 which is coupled to the silica has reacted further with $3 \mathrm{~m} 1 \mathrm{p}$, the additional olefin part should contribute to enhance further the hydrophobation effect on the silica surface. Furthermore, if the addition reaction product between Si 263 and 3m1p has not reacted with the silica surface, the hydrophobation effect should not be enhanced. Therefore, the 
following experiment was carried out in order to check if there is a difference in terms of the hydrophobation degree on the silica surface between the systems with and without $3 \mathrm{~m} 1 \mathrm{p}$.

Adding distilled water to the system after heating can separate the silica into two phases depending on the hydrophobation degree by the following mechanism. Water and $3 \mathrm{~m} 1 \mathrm{p}$ or decane are different in terms of specific gravity and degree of polarity, therefore $3 \mathrm{~m} 1 \mathrm{p}$ and decane are both floating on water. The silica with its surface modified by Si 263 or Si 263 together with $3 \mathrm{~m} 1 \mathrm{p}$ by a chemical bonding is expected to be present in the decane and $3 \mathrm{~m} 1 \mathrm{p}$ phase due to the increased hydrophobicity. The unmodified silica is hydrophilic, so silica will soon sink in water to the bottom. As a result, the water layer is in the middle. The separated layers in water of the above described samples before and after heating at $150^{\circ} \mathrm{C}$ for 30 or 60 minutes are shown in Figure 4-4 (left). As the silica in both systems can be hydrophobized by Si 263 , the reduction of the amount of silica in the bottom layer with increasing heating time can be observed. However, the trend is more significant in the sample with $3 \mathrm{~m} 1 \mathrm{p}$. This tendency can be also confirmed by measuring the silica weights in the top and bottom layers separately (Figure 4-4 (right)). A higher proportion of silica in the top layer implies that there is a greater extent of modification on the silica surface. This result supports additionally the formation of the direct silica - Si 263 - 3m1p bonds in the system.

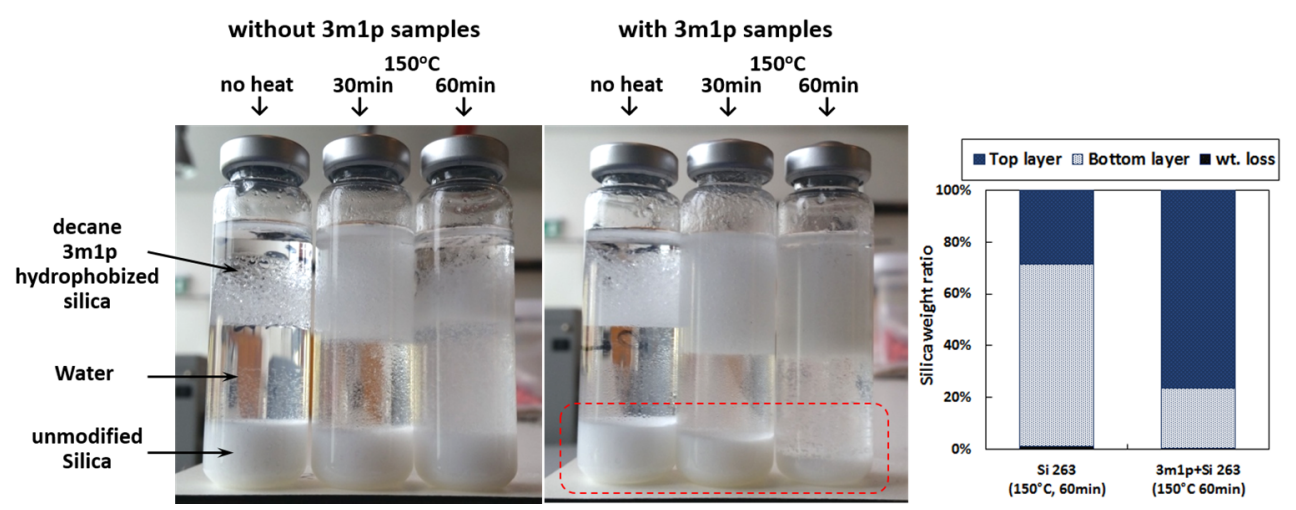

Figure 4-4: (left) Separated layers with water of mixtures of silica, Si 263 and decane with or without $3 \mathrm{~m} 1 \mathrm{p}$ before and after heating at $150^{\circ} \mathrm{C}$ for 30 or 60 minutes, (right) silica weight ratio in the top or bottom layer of the sample with or without $3 \mathrm{~m} 1 \mathrm{p}$ after heating at $150{ }^{\circ} \mathrm{C}$ for 60 minutes 
The above two reasons (no addition reaction product peak in the GC chromatogram and an enhanced hydrophobation effect on the silica surface) strongly suggest that most of the Si 263 which is coupled to $3 \mathrm{~m} 1 \mathrm{p}$ have also coupled to the silica surface. There is one more interesting point to mention: The total amount of silane which has reacted with silica increased compared to the system without $3 \mathrm{~m} 1 \mathrm{p} \mathrm{(22 \%} \mathrm{in} \mathrm{the} \mathrm{silica} \mathrm{/} \mathrm{silane} \mathrm{system,} 28 \%$ in the $3 \mathrm{~m} 1 \mathrm{p} /$ silica / silane system). As this measurement was done only once, the measurement error has to be considered. However, if this difference is true, it means that the silanization reaction possibility is increased in the presence of olefin. It is difficult to find the exact reason for this enhancement from the experiments done in this study, but one of the possible reasons for it can be the different solubilities of Si 263 and the addition reaction product in decane. The addition reaction product could be less soluble in decane compared to Si 263 because of the bigger size. Therefore, the addition reaction product tends to get segregated from the decane phase which results in a higher possibility to be adsorbed at the silica surface. This mechanism might be the first hint to explain the higher silanization rate in the presence of $3 \mathrm{~m} 1 \mathrm{p}$ in this solution system. The other possibility is also related to an increased molecular weight by the addition reaction of Si 263 to $3 \mathrm{~m} 1 \mathrm{p}$. The bigger size reduces the free movement of the molecule, then this restricted rotation might enable an easier adsorption at the silica surface by keeping the attached structure. Or, the reactivity of the ethoxy part of the silane might be boosted by being reacted by $3 \mathrm{~m} 1 \mathrm{p}$.

In any case, the formation of the direct silica - silane - olefin bonds has contributed to the significantly enhanced hydrophobation effect on the silica surface, which seems to be one of the main reasons for the improved achievable silica dispersion level in the mercaptosilane system.

\section{4-3-2 Influence of $\mathrm{ZnO}$ on the olefin / Si 263 reaction}

It is well known that ZnO plays a very important role in the vulcanization stage. It forms a complex with accelerators, subsequently incorporates sulfur inside, which results in the creation of active sulfurating agents. This process leads to an increase in the vulcanization efficiency. As the reaction of poly-sulfide silanes with rubber is also a sulfur involving reaction, the activation effect for sulfur moieties released from the silane by $\mathrm{ZnO}$ has been considered as one of the reasons for the premature scorch at high temperatures.[4] Therefore, the influence of $\mathrm{ZnO}$ on the reaction between mercapto-silane and rubber was 
also investigated. In this subsection, the observed behavior of the 3m1p / Si 263 system involving $\mathrm{ZnO}$ will be summarized.

Figure 4-5 shows the concentration change of 3m1p and Si 263 in the samples with and without $\mathrm{ZnO}$ by heating at $150^{\circ} \mathrm{C}$. In the presence of $\mathrm{ZnO}$, on the one hand the reduction of Si 263 is accelerated, on the other hand the reduction of $3 \mathrm{~m} 1 \mathrm{p}$ is suppressed compared to those in the sample without $\mathrm{ZnO}$. Despite the fact that the GC measurement of the unheated sample was conducted directly after the preparation of the sample, the Si 263 peak area shows already a lower value than the expected value from the initial concentration. From the GC chromatogram, the generation of the disulfide-silane was confirmed as a resulting new product (Figure 4-6). This means that Si 263 interacts with ZnO even at room temperature, which results in the formation of the disulfide-silane.
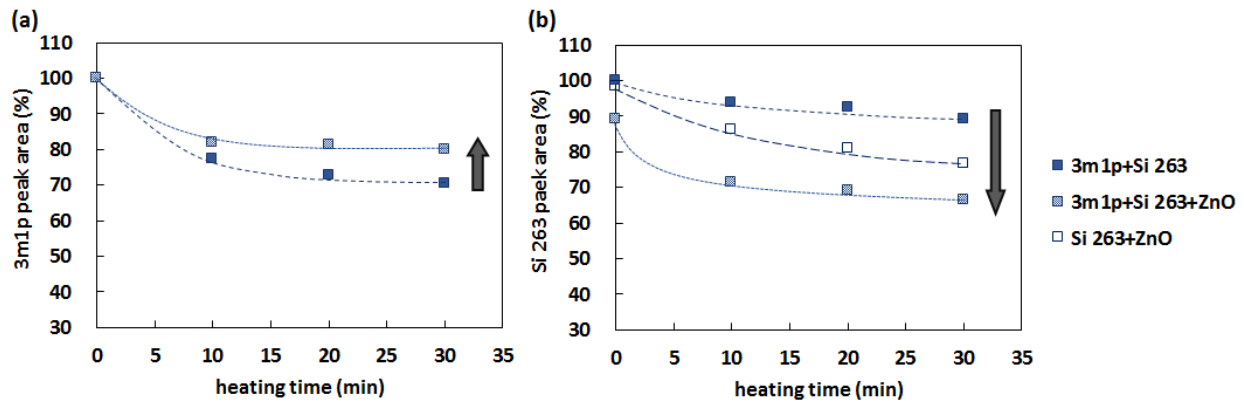

Figure 4-5: Change of the (a) $3 \mathrm{~m} 1 \mathrm{p}$ and (b) Si 263 concentration with heating time in the 3m1p / Si 263, 3m1p / Si 263 / ZnO and Si 263 / ZnO systems

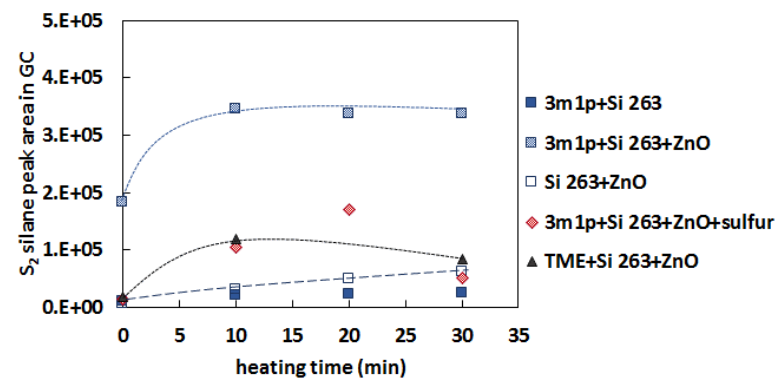

Figure 4-6: Disulfide-silane peak area change with heating time 
Mercaptobenzothiazol (MBT), which has also a mercapto group, is known to form a complex with $\mathrm{ZnO}$ as shown in Figure 4-7,[6,7,8] which can be converted into the active sulfating complex. Based on this mechanism, it can be assumed that mercapto-silanes can be activated by $\mathrm{ZnO}$ in the same way, which consequently results in the formation of the complex shown in Figure 4-8.

2

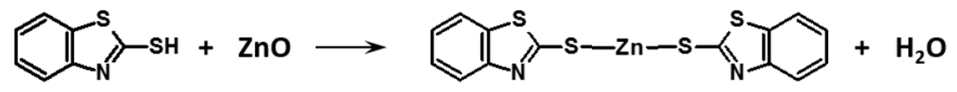

Figure 4-7: Reaction between $\mathrm{MBT}$ and $\mathrm{ZnO}$

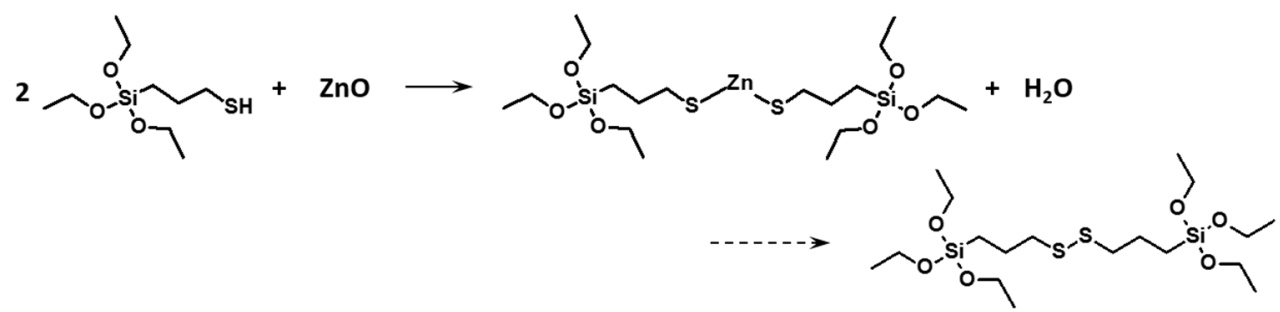

Figure 4-8: Possible reaction between Si 263 and ZnO

This is supported by the phenomenon observed when elemental sulfur is added additionally to the system (Figure 4-6). In this case, the generation of disulfide-silane is lower than in the absence of sulfur. Additionally, a large amount of trisulfide-silane is observed in the GC measurement (not shown). The MBT-Zn complex is known to accelerate the ring-opening of the elemental sulfur,[9] therefore, it can be assumed that the Si 263 Zn complex reacts in the same way. It can incorporate sulfur radicals, and, as a result, some parts of silanes are stabilized as trisulfide-silanes.

In the sample without sulfur, it becomes that the concentration of disulfide-silane doesn't change after reaching a certain level even with increasing heating time (Figure 4-6). There are two possibilities to interpret this behavior. As a first one, the production of disulfidesilane has just stopped at a certain point. The second one is that the generation of disulfidesilane has continued, however it has also reacted with $3 \mathrm{~m} 1 \mathrm{p}$ in a similar rate, which results in showing the constant concentration. An additional experiment using the mixture of $3 \mathrm{~m} 1 \mathrm{p}$ and $\mathrm{Si}$ 75, which contains the disulfide-silane as the main component, shows no concentration change of both disulfide-silanes and $3 \mathrm{~m} 1 \mathrm{p}$ after heating at $150^{\circ} \mathrm{C}$. It has also been reported in the literature that disulfide-silane doesn't react with squalene without 
incorporating sulfur inside the structure.[10] This explains that the second possibility is unlikely in this case, and this can explain the suppressed decrease of $3 \mathrm{~m} 1 \mathrm{p}$ in the $\mathrm{ZnO}$ involving system compared to the system without $\mathrm{ZnO}$ (Figure 4-5), as well. The reason that the disulfide-silane content does not increase might be because of the reduction of the available $\mathrm{ZnO}$ due to the formation of the Si 263-Zn complex layer on the $\mathrm{ZnO}$ surface. Actually, it has been reported that $\mathrm{ZnO}$ does not react completely with MBT by this mechanism.[11]

Furthermore, the tendencies that the generation of disulfide-silane is accelerated by the presence of olefins and also the acceleration degree varies depending on the type of olefin were observed (Figure 4-6). In the presence of an olefin, not only the enhanced decrease of Si 263, but also the large conversion of Si 263 finally to the disulfide-silane were noticed. The clear mechanism is unknown, but it seems that olefins contribute to the step of the conversion from the complex to disulfide-silane in Figure 4-8.

Transferring this knowledge to real rubber compounds, the change of the feeding time of $\mathrm{ZnO}$ may alter the reaction degree between the mercapto group of the silane and the polymer resulting in the better balance of compound properties and processability.

\section{4-3-3 Influence of CBS or sulfur on the olefin / Si 263 reaction}

It has been reported in the literature that mercapto-silanes react stoichiometrically with the accelerator CBS even at room temperature in a model system, which results in the formation of disulfide-silanes, MBT and cyclohexylamine (Figure 4-9).[12] This reaction process seems to contain a radically induced generation of the mercapto-silane. If the mercapto-silane radicals are produced in the CBS involving system, the radicals seem to have two possibilities, to react either with the double bonds in $3 \mathrm{~m} 1 \mathrm{p}$ or with CBS. Therefore, an investigation in the 3m1p / Si 263 / CBS system was carried out in order to know the preferred process leading to the formation of a disulfide-silane or the addition reaction product with $3 \mathrm{~m} 1 \mathrm{p}$. The influence of elemental sulfur was also evaluated.

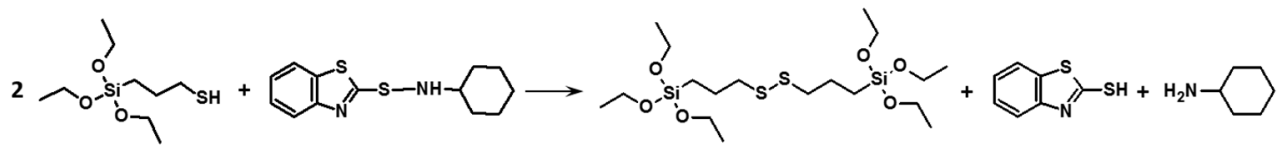

Figure 4-9: Reaction between Si 263 and CBS 
Figure 4-10 shows the concentration change of 3m1p and Si 263 measured by the GC measurement. Similar to the $\mathrm{ZnO}$ containing system, the reduction of Si 263 even before heating together with the generation of disulfide-silane was observed (Figure 4-11). The conversion is more pronounced in the $3 \mathrm{~m} 1 \mathrm{p} / \mathrm{Si} 263$ / CBS system compared to the $3 \mathrm{~m} 1 \mathrm{p} /$ Si 263 / ZnO system. The generated disulfide-silane is again stable, as a result, $3 \mathrm{~m} 1 \mathrm{p}$ has kept the original concentration (Figure 4-10).
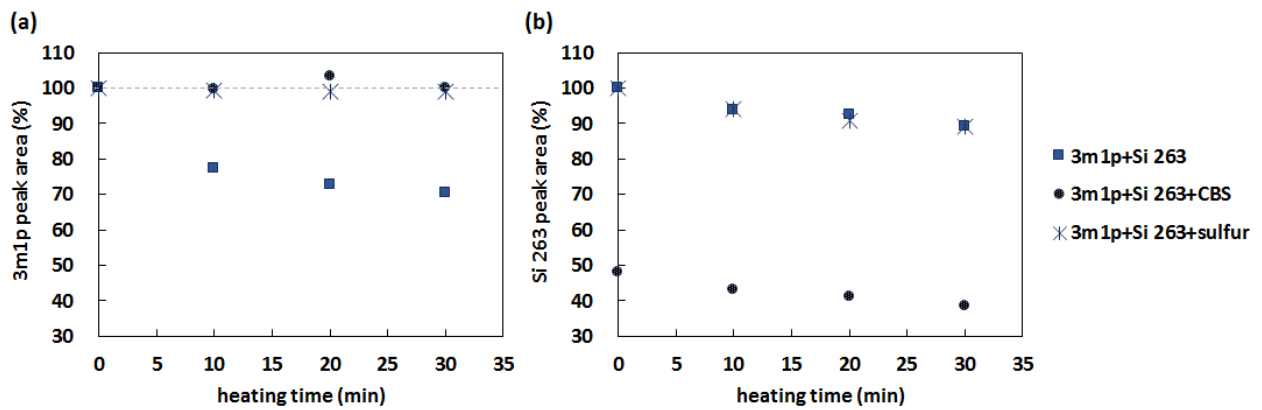

Figure 4-10: Change of the $3 \mathrm{~m} 1 \mathrm{p}$ and Si 263 concentration with heating time in the $3 \mathrm{~m} 1 \mathrm{p} /$ Si 263, 3m1p / Si 263 / CBS and 3m1p / Si 263 / sulfur systems

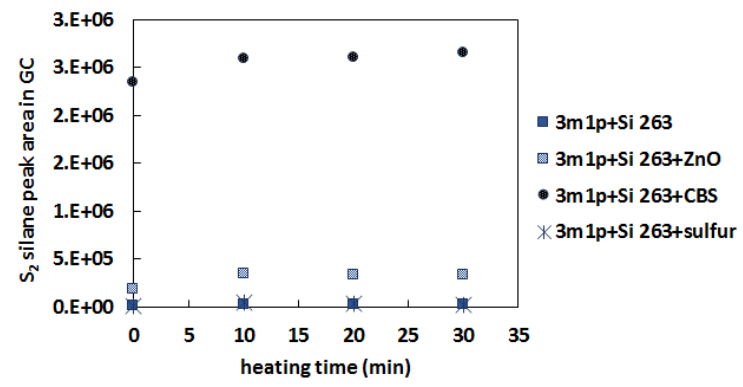

Figure 4-11: Disulfide silane peak area change with heating time

When adding sulfur, the formation of disulfide-silane as well as trisulfide-silane was confirmed. Mercapto-silane radicals can react with sulfur which result in a radical with an extra sulfur atom. This new radical can react with another mercapto-silane radical, resulting in a trisulfide-silane, as shown in Figure 4-11. Again, no reduction of the concentration of $3 m 1 p$ is observed (Figure 4-10). 


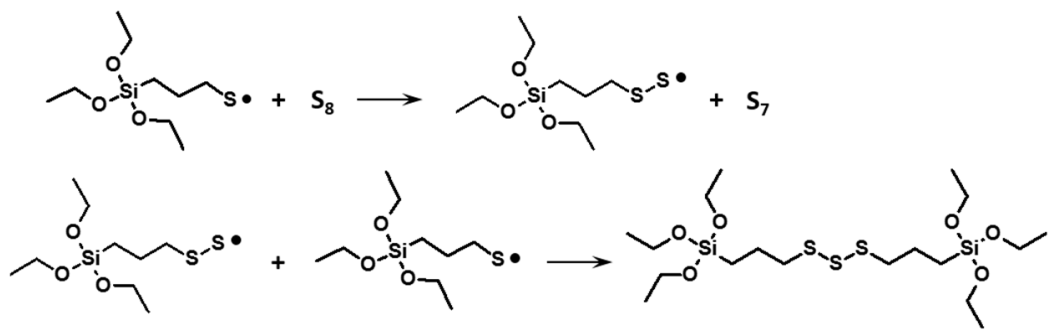

Figure 4-11: Possible generation mechanism of trisulfide-silane in the Si 263 / sulfur system

Therefore, considering the actual rubber system, it can be assumed that adding CBS or small amounts of elemental sulfur at the $1^{\text {st }}$ mixing stage for the mercapto-silane / silica filled compounds alters the influencing degree of mercapto-silane to rubber which was already discussed in Chapter 3 (addition reaction and allylic hydrogen abstraction). Changing the mercapto-silane into disulfide and / or trisulfide-silane may have just a similar influence as using the reduced amount of the mercapto-silane in combination with disulfide and / or trisulfide-silane. However, it has been reported in the literature that when the mercapto-silanes which already coupled to the silica react further with CBS, the intermediate can be stabilized on the silica surface without converting to the disulfide-silane (Figure 4-12).[12] Therefore, depending on the feeding time of CBS, a different effect from that one, which occurs in the combined addition of mercapto and disulfide-silane, might be obtained. In any case, it seems to be worth carrying out additional rubber compound tests to check this effect.

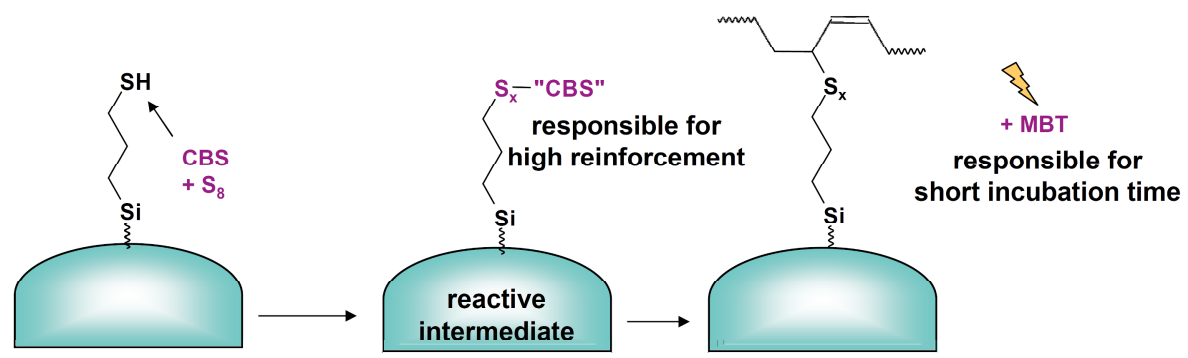

\section{Immobilization of mercaptosilane at silica surface prevents it from dimerization to disulfide}

Figure 4-12: Already reported immobilization mechanism of mercapto-silane reacted with CBS [12] 


\section{4-4 Conclusions}

In Chapter 3, it was figured out that mercapto-silanes react with the double bond of olefins, especially with a vinyl double bond, following a radical addition reaction. This fact indicates that mercapto-silanes give a different reinforcing structure in silica-filled compounds compared to sulfide-silanes. In this chapter, the influence of further ingredients like silica, $\mathrm{ZnO}, \mathrm{CBS}$ and sulfur on the addition reaction was investigated using the $3 \mathrm{~m} 1 \mathrm{p} /$ Si 263 system.

Firstly, the behavior in the presence of silica was investigated. The following three observations were made leading to the conclusion that Si 263 reacts with $3 \mathrm{~m} 1 \mathrm{p}$ also in the presence of silica, and that most of the addition reaction products were also coupled to the silica surface following a silanization reaction. The first observation was the decrease of the $3 \mathrm{~m} 1 \mathrm{p}$ concentration in the silica system. The second one was the observed higher amount of modified silica in the system with $3 \mathrm{~m} 1 \mathrm{p}$ compared to the one without $3 \mathrm{~m} 1 \mathrm{p}$, which was obtained by investigating the behavior of (treated) silica in the presence of water. The third one was regarding the peak of the addition reaction product between $3 \mathrm{~m} 1 \mathrm{p}$ and Si 263 in the GC chromatogram. The peak was observed for the sample without silica as shown in Chapter 3, but it was not present in the system with silica despite a decrease of the amount of $3 \mathrm{~m} 1 \mathrm{p}$. These three observations support the formation of the direct silica - silane - olefin bonds in the system, and this structure seems to contribute to enhance the hydrophobation effect on the silica surface resulting in one of the main reasons for the improved achievable silica dispersion level in the mercapto-silane system.

Next, adding ZnO or CBS to the 3m1p / Si 263 system leads to a similar effect but to a different degree following different mechanisms. The decrease of Si 263 was accelerated in the presence of $\mathrm{ZnO}$ or CBS, and the resulting product was a disulfide-silane. This tendency is more pronounced in the presence of CBS, and it was judged that almost all added CBS were used for the reaction with Si 263 even without heating. Disulfide-silanes do not react with $3 \mathrm{~m} 1 \mathrm{p}$ directly unless elemental sulfur was added. As a consequence, the amount of $3 \mathrm{~m} 1 \mathrm{p}$ did not decrease in the system with CBS, therefore, it was considered that no addition reaction of Si 263 with $3 \mathrm{~m} 1 \mathrm{p}$ has occurred. In the system with $\mathrm{ZnO}$, the decrease of $3 \mathrm{~m} 1 \mathrm{p}$ was still observable, but the rate was rather slow, so it is reasonable to conclude that the $3 \mathrm{~m} 1 \mathrm{p} / \mathrm{Si} 263$ reaction was suppressed by the effect of $\mathrm{ZnO}$. When adding sulfur to the $3 \mathrm{~m} 1 \mathrm{p} / \mathrm{Si} 263$ system, the generation of disulfide as well as trisulfide-silane has priority, and 
no decrease of $3 \mathrm{~m} 1 \mathrm{p}$ was observed. Although the degree and the mechanism are different, it became clear that involving $\mathrm{ZnO}, \mathrm{CBS}$ or sulfur in the mercapto-silane system suppressed to generate the addition reaction product between the mercapto-silane and the olefin. Assuming the reaction mechanism in a real rubber compound, the usage of these ingredients (ZnO, CBS and / or sulfur) might also lead to a reduction of the Si 263 / rubber reaction degree during mixing of the compounds. Therefore, it could decrease the amount of bound rubber or the radical related reactions triggered by the mercapto-silane radicals. If the reason for worse processability in the mercapto-silane system is the excess reaction between the mercapto-silane and the rubber, these ingredients might have possibility to change the resulting situations. It has to be considered that the usage of sulfur can have a risk for an excess of the cross-linking reaction if it is used in a too high amount. However, the reaction degree between mercapto-silane and rubber could be controlled by changing the feeding time and the feed amount of the above mentioned additional ingredients, therefore, it seemed to be worth to investigate these effects further in the rubber compound system. 


\section{4-5 References}

[1] S. Mihara, "Reactive processing of silica-reinforced tire rubber: new insight into the time and temperature-dependence of silica rubber interaction", PhD. Thesis: 2009, Dept. of Elastomer Technology and Engineering, Univ. of Twente, Enschede, the Netherlands.

[2] W. Kaewsakul, "Silica-Reinforced Natural Rubber for Low Rolling Resistance, EnergySaving Tires", Thesis: 2013, Dept. Rubber Technol., Univ. of Twente, Enschede, the Netherlands

[3] C. Hayichelaeh, L.A.E.M. Reuvekamp, W.K. Dierkes, A. Blume, J.W.M. Noordermeer, K. Sahakaro, Rubber Chem. Technol., 90, (2017) 651-666

[4] L.A.E.M Reuvekamp, "Reactive mixing of silica and rubber for tires and engine mounts", Thesis: 2003, Dept. Rubber Technol., Univ. of Twente: Enschede, the Netherlands

[5] A. Blume, M. El-Roz, F. Thibault-Starzyk, Kautsch. Gummi Kunstst., 10 (2013) 63 - 70

[6] G. Heideman, "Reduced Zinc Oxide Levels in Sulphur Vulcanization of Rubber Compounds", Thesis: 2004, Dept. Rubber Technol., Univ. of Twente, Enschede, the Netherlands

[7] A.Y. Coran, Rubber Chem. Technol., 37 (1964) 679

[8] M.H.S. Gradwell, W.J. McGill, J. Appl. Polym. Sci., 58 (1995) 2193

[9] H. Shizuka, T. Azami, Journal of the Society of Rubber Science and Technology, Japan, 40 (1967) 467-471

[10] A. Hasse, H.-D. Luginsland, International Rubber Conference 2000, Helsinki, June 1215 (2000)

[11] M.H.S. Gradwell, W.J. McGill, J. Appl. Polym. Sci., 61 (1996) 1131-1136

[12] O. Klockmann, J. Hahn, H. Scherer, International Rubber Conference 2009, Nuremberg, Germany, June 29-July 2 (2009) 


\section{Chapter 5}

\section{Model compound study-3; \\ Reactivity study of mercapto-silane and sulfide-silane with squalene or liquid butadiene}

\section{5-1 Introduction}

Carrying out model studies using low molecular-weight model compounds such as olefins to understand the vulcanization reaction, has two main disadvantages: The first one is the high concentration of end-groups of the compounds. These end-groups (mostly methyl groups) have a different reactivity compared to the methylene groups which form the majority of reactive groups in a rubber. The other disadvantage is that olefins are monofunctional, i.e. they have only one double bond in the molecule. Gregg and Katrenick have even stated that for the accurate modeling of the BR vulcanization a minimum of three double bonds are necessary.[1] Since the actual rubber has a lot of conjugated double bonds in the molecule, an investigation using model compounds which have a relatively high molecular-weight should enable a much more accurate understanding of the phenomena in the actual rubber system.

However, there is the problem that the usage of higher molecular weight compounds causes a limitation of the available analytical methods. Especially, it is difficult to obtain a high resolution spectra from high molecular weight materials such as polymers in the solidstate NMR because of strong dipolar - dipolar interactions. Therefore, to use a material which is applicable for the solution-state NMR is desirable. As a good compromise, various studies have been carried out by using squalene or butadiene oligomers ( $\approx$ liquid BR) which are still liquid, even after the vulcanization reaction, but have some double bonds in the molecule. For these samples, LC/MS, GPC and solution-state NMR are still applicable.

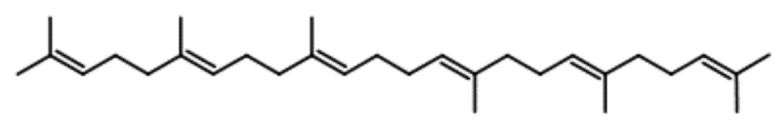

Figure 5-1: Structure of squalene 
Squalene is a convenient model substance also in terms of availability and handling. It contains six double bonds and could be considered as a very good model for NR / IR, although there is one important difference because all double bonds in squalene are transconfigured and not cis as in NR and IR. Borrós et al. have e.g. already carried out many studies using squalene to identify the intermediate structures as well as the cross-linking products.[2-4]

Another liquid polymer as model substance was already used by Hahn et al.. They used Polyoil 110 (butadiene oligomer of about 44 monomers, cis / trans / vinyl = 72 / $27 / 1$ ) to identify successfully some products of the accelerated sulfur vulcanization. They also confirmed that the ${ }^{13} \mathrm{C}$ NMR spectrum of Polyoil 110 vulcanizates and the solid state ${ }^{13} \mathrm{C}$ spectrum of BR vulcanizates have strong similarities, therefore, the peak assignments seem to be valid for both spectra.[5]

In this chapter, a model compound study using squalene and liquid BR was carried out in order to know if the similar tendencies as seen in the model olefin study can be obtained in the systems which are much closer to the actual rubber system.

\section{5-2 Experimental}

\section{5-2-1 Sample preparation}

The used materials in this study are summarized in Table 5-1.

Table 5-1; Material list for the model olefin study

\begin{tabular}{cccc}
\hline Material & Source & $\begin{array}{c}\text { 1,2-vinyl } \\
\text { content (\%) }\end{array}$ & $\begin{array}{c}\text { Molecular weight } \\
\text { (g/mol) }\end{array}$ \\
\hline squalene & Wako & - & 410.73 \\
Ricon 156 & Cray Valley & 70 & $\left.1400^{*}\right)$ \\
Ricon 130 & Cray Valley & 28 & $\left.2500^{*}\right)$ \\
\hline (3-Mercaptopropyl)triethoxysilane (Si 263) & Evonik & - & 238.42 \\
bis(triethoxysilylpropyl)-tetrasulfide (Si 69) & Evonik & - & 532.00 \\
\hline Decane & Sigma-Aldrich & - & 142.28 \\
\hline & & ${ }^{*}$ number average molecular weight
\end{tabular}


Before the preparation of the samples, all of materials were flushed with nitrogen. In the case of a squalene system, an isomolar mixture of a silane and squalene was weighed for the identification of reaction products. Twice as much mole of decane than squalene and silane was added for the quantitative measurements as an internal standard. For the case of liquid polymer system, firstly, the number of the double bond in a molecule was calculated by dividing the number average molecular weight by the molecular weight of a butadiene monomer unit which is 54 . Then, a silane and a liquid polymer were weighed to satisfy that a number of double bond of a liquid polymer and a silane become an isomolar content. The mixture was divided into different small glass tubes $(2.0 \mathrm{ml})$. The reaction was carried out by placing the tubes in a device named PL-SP 260VS which can shake and heat. Each tube was heated up to the desired specific temperature for pre-determined durations. After the reaction, the tubes were cooled immediately in an ice bath to stop the reaction. Samples were prepared for the further chemical analysis.

\section{5-2-2 Analytical methods}

The Liquid Chromatography - Mass spectrometry (LC/MS) analysis and the Nuclear Magnetic Resonance (NMR) spectroscopy analysis were carried out following the same conditions as shown in Chapter 3 . The ${ }^{1} \mathrm{H}$ and ${ }^{13} \mathrm{C}$ chemical shift were calculated by using the commercially available software program ACD/C+H NMR (Version 12.0).

\section{5-3 Results and Discussion}

\section{5-3-1 Reaction of squalene with two different silanes}

As mentioned in the introduction, squalene is an oligomer which has six isoprene units with trans configuration. The investigation with this molecule should help to understand the observed phenomena in the NR or IR rubber compounds. Based on the information obtained in the model olefin study with $\mathrm{T3M} 3 \mathrm{H}$ which also has the isoprene type of structure, the presence or absence of isomers, addition reaction products of silane to squalene and cross-linked products especially in the Si 69 system, were investigated.

Figure 5-2 shows the ${ }^{1} \mathrm{H}$ NMR spectra of the squalene / Si 263 system before and after heating at $170{ }^{\circ} \mathrm{C}$ for 60 minutes. It looks like there is no big change of the spectra after heating at first glance. However, as can be recognized in the expanded areas with 
overlapping two spectra, the peak shapes around 2.0 and $5.1 \mathrm{ppm}$, which correspond to the allylic hydrogen and the double bond respectively, show different shapes before and after heating. This implies that an isomerization is the reaction which occurs mainly in the system.

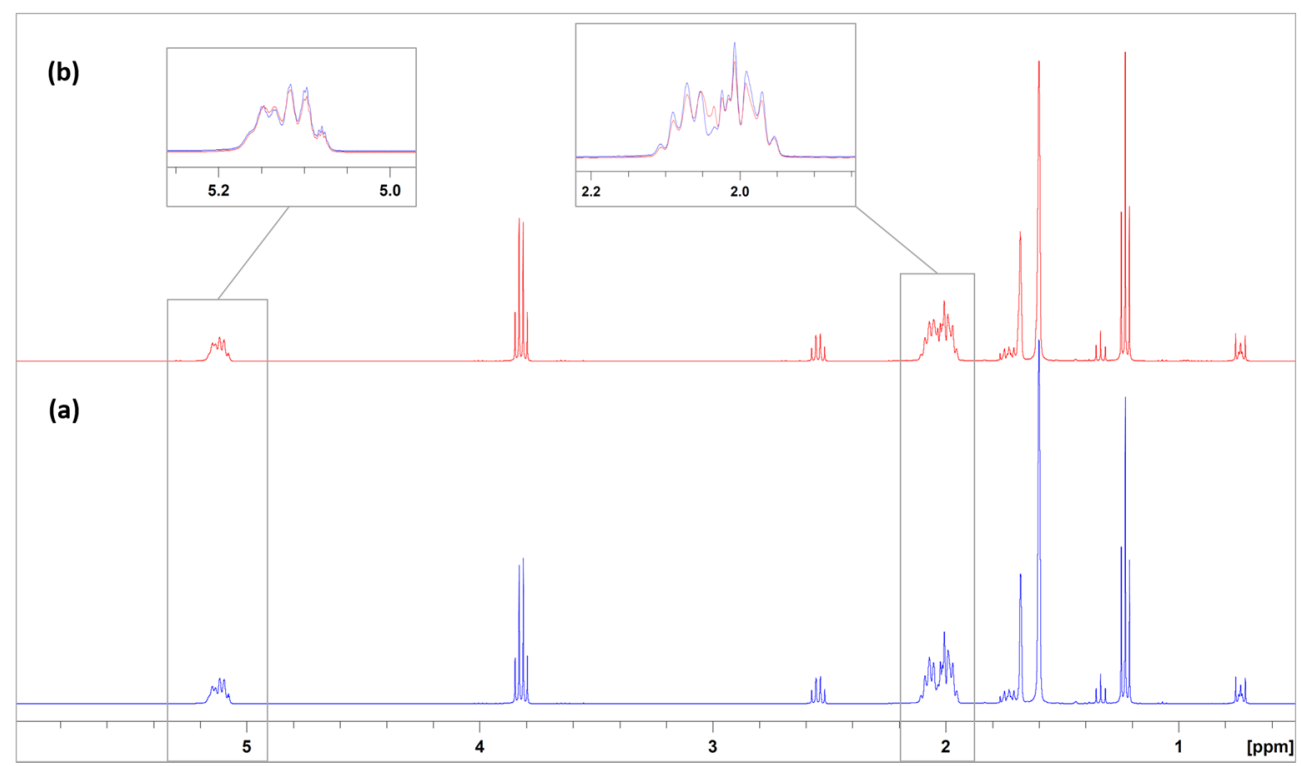

Figure 5-2: ${ }^{1} \mathrm{H}$ NMR of the squalene / Si 263 mixture (a) before and (b) after heating at 170 ${ }^{\circ} \mathrm{C}$ for 60 minutes

Figure 5-3 shows the ${ }^{13} \mathrm{C}$ NMR spectra of the same samples. Many small peaks have arisen after heating, however, most of them appear in the surrounding of the original peak positions of squalene, except the peaks around 23 and $32 \mathrm{ppm}$. Firstly, the chemical shifts of the expected structures as addition products were calculated by the prediction software. After that, it was checked if the new peaks were observed in the expected places after heating. However, it seemed that the expected peaks have not been observed. Secondary, the presence of isomers was analyzed in the following way. As squalene has four trans double bonds inside plus two external double bonds, several structures can be expected as isomers depending on how many trans double bonds change to cis double bonds, even in the case that the shift of the double bond (allylic rearrangement) is not considered. The chemical shifts of possible isomers were calculated by the prediction software and summarized in Table 5-2. 
(b)

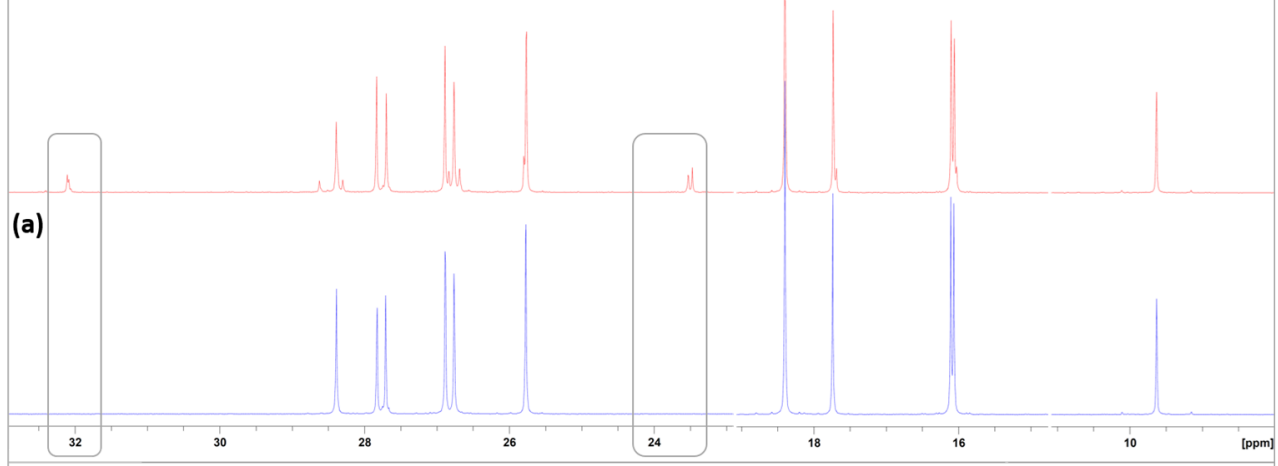

(b)

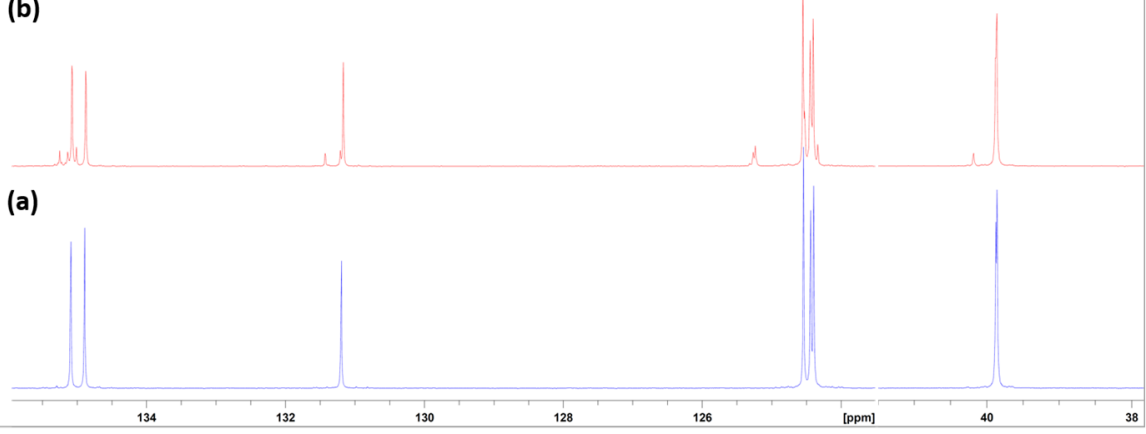

Figure 5-3: ${ }^{13} \mathrm{C}$ NMR of the Squalene / Si 263 mixture (a) before and (b) after heating at 170 ${ }^{\circ} \mathrm{C}$ for 60 minutes 
Table 5-2: Calculated chemical shift of isomers of squalene

\begin{tabular}{|c|c|c|c|c|c|c|c|c|c|c|c|c|c|c|c|c|c|c|c|}
\hline \multicolumn{2}{|c|}{ structure } & \multicolumn{2}{|c|}{-TाT-(Squalene) } & \multicolumn{2}{|c|}{$-\pi \Pi \mathrm{C}-$} & \multicolumn{2}{|c|}{-ПТС- } & \multicolumn{2}{|c|}{-ாतC- } & \multicolumn{2}{|c|}{-TCCT- } & \multicolumn{2}{|c|}{-TCCC- } & \multicolumn{2}{|c|}{-СтC- } & \multicolumn{2}{|c|}{ - СTCC- } & \multicolumn{2}{|c|}{- CCCC- } \\
\hline No. & & ${ }^{13} \mathrm{C}$ & ${ }^{1} \mathrm{H}$ & ${ }^{13} \mathrm{C}$ & ${ }^{1} \mathrm{H}$ & ${ }^{13} \mathrm{C}$ & ${ }^{1} \mathrm{H}$ & ${ }^{13} \mathrm{C}$ & ${ }^{1} \mathrm{H}$ & ${ }^{13} \mathrm{C}$ & ${ }^{1} \mathrm{H}$ & ${ }^{13} \mathrm{C}$ & ${ }^{1} \mathrm{H}$ & ${ }^{13} \mathrm{C}$ & ${ }^{1} \mathrm{H}$ & ${ }^{13} \mathrm{C}$ & ${ }^{1} \mathrm{H}$ & ${ }^{13} \mathrm{C}$ & ${ }^{1} \mathrm{H}$ \\
\hline 9 & $-\mathrm{CH}_{3}$ & 25.72 & 1.68 & 25.72 & 1.68 & 25.72 & 1.68 & 25.72 & 1.68 & 25.72 & 1.68 & 25.72 & 1.68 & 25.72 & 1.68 & 25.72 & 1.68 & 25.72 & 1.68 \\
\hline 13 & $-\mathrm{CH}_{3}$ & 17.65 & 1.61 & 17.65 & 1.61 & 17.65 & 1.61 & 17.65 & 1.61 & 17.65 & 1.61 & 17.65 & 1.61 & 17.65 & 1.61 & 17.65 & 1.61 & 17.65 & 1.61 \\
\hline 8 & $-\mathrm{C}$ & 131.03 & - & 131.03 & - & 131.03 & - & 131.03 & - & 131.03 & - & 131.03 & - & 130.60 & - & 130.60 & - & 130.60 & - \\
\hline 7 & $-\mathrm{CH}$ & 124.70 & $\begin{array}{r}5.07 \\
-5.13\end{array}$ & 124.70 & $\begin{array}{r}5.07 \\
-5.13\end{array}$ & 124.70 & $\begin{array}{c}5.07 \\
-5.13\end{array}$ & 124.70 & $\begin{array}{r}5.07 \\
-5.11\end{array}$ & 124.70 & $\begin{array}{r}5.07 \\
-5.13\end{array}$ & 124.70 & $\begin{array}{c}5.07 \\
-5.13\end{array}$ & 124.80 & $\begin{array}{c}5.07 \\
-5.13\end{array}$ & 124.80 & $\begin{array}{r}5.08 \\
-5.13\end{array}$ & 124.80 & $\begin{array}{r}5.07 \\
-5.13\end{array}$ \\
\hline \multirow[t]{2}{*}{6} & $-\mathrm{CH}_{2}$ & 26.90 & $\begin{array}{r}1.92 \\
-1.99\end{array}$ & 26.90 & $\begin{array}{r}1.92 \\
-1.99\end{array}$ & 26.90 & $\begin{array}{c}1.92 \\
-2.00\end{array}$ & 26.90 & $\begin{array}{r}1.92 \\
-2.00\end{array}$ & 26.90 & $\begin{array}{r}1.92 \\
-2.00\end{array}$ & 26.90 & 1.97 & 27.00 & $\begin{array}{r}2.05 \\
-2.06\end{array}$ & 27.00 & $\begin{array}{r}2.03 \\
-2.07\end{array}$ & 27.00 & $\begin{array}{r}2.03 \\
-2.07\end{array}$ \\
\hline & & \multicolumn{2}{|c|}{ Trans } & \multicolumn{2}{|c|}{ Trans } & \multicolumn{2}{|c|}{ Trans } & \multicolumn{2}{|c|}{ Trans } & \multicolumn{2}{|c|}{ Trans } & \multicolumn{2}{|c|}{ Trans } & \multicolumn{2}{|c|}{ Cis } & \multicolumn{2}{|c|}{ Cis } & \multicolumn{2}{|c|}{ Cis } \\
\hline 5 & $-\mathrm{CH}_{2}$ & 39.90 & $\begin{array}{c}1.92 \\
-1.99\end{array}$ & 39.90 & $\begin{array}{r}1.94 \\
-1.99\end{array}$ & 39.90 & $\begin{array}{r}1.92 \\
-1.99\end{array}$ & 39.90 & $\begin{array}{r}1.93 \\
-1.99\end{array}$ & 39.90 & $\begin{array}{c}1.93 \\
-1.99\end{array}$ & 39.90 & 1.95 & 31.92 & $\begin{array}{r}2.03 \\
-2.05\end{array}$ & 31.92 & $\begin{array}{c}2.03 \\
-2.07\end{array}$ & 31.92 & $\begin{array}{r}2.03 \\
-2.07\end{array}$ \\
\hline 12 & $-\mathrm{CH}_{3}$ & 15.95 & 1.60 & 15.95 & $\begin{array}{r}1.59 \\
-1.61\end{array}$ & 15.95 & 1.60 & 15.95 & $\begin{array}{r}1.59 \\
-1.61\end{array}$ & 15.95 & $\begin{array}{r}1.59 \\
-1.61\end{array}$ & 15.95 & 1.60 & 23.35 & 1.68 & 23.35 & 1.68 & 23.35 & 1.68 \\
\hline 4 & $-\mathrm{C}$ & 134.85 & - & 134.85 & - & 134.85 & - & 134.85 & - & 135.35 & - & 135.35 & - & 135.05 & - & 135.05 & - & 135.05 & - \\
\hline 3 & $-\mathrm{CH}$ & 124.60 & $\begin{array}{r}5.09 \\
-5.15\end{array}$ & 124.60 & $\begin{array}{r}5.09 \\
-5.15\end{array}$ & 124.60 & $\begin{array}{r}5.09 \\
-5.15\end{array}$ & 124.60 & $\begin{array}{r}5.09 \\
-5.15\end{array}$ & 124.32 & $\begin{array}{c}5.02 \\
-5.07\end{array}$ & 124.32 & $\begin{array}{r}5.02 \\
-5.07\end{array}$ & 124.82 & $\begin{array}{c}5.04 \\
-5.10\end{array}$ & 124.82 & $\begin{array}{c}5.04 \\
-5.10\end{array}$ & 124.82 & $\begin{array}{r}5.07 \\
-5.13\end{array}$ \\
\hline \multirow[t]{2}{*}{2} & $-\mathrm{CH}_{2}$ & 26.90 & $\begin{array}{r}2.05 \\
-2.11\end{array}$ & 26.90 & $\begin{array}{r}2.05 \\
-2.13 \\
\end{array}$ & 26.49 & $\begin{array}{r}2.07 \\
-2.13\end{array}$ & 26.49 & $\begin{array}{r}2.05 \\
-2.13\end{array}$ & 26.35 & $\begin{array}{r}2.04 \\
-2.12\end{array}$ & 26.35 & $\begin{array}{r}2.05 \\
-2.09\end{array}$ & 26.12 & $\begin{array}{r}2.01 \\
-2.06\end{array}$ & 26.12 & $\begin{array}{r}2.00 \\
-2.08\end{array}$ & 26.76 & $\begin{array}{r}1.94 \\
-2.00\end{array}$ \\
\hline & & & & & & Tra & & & & & & & & Tra & & & & ci & \\
\hline 1 & $-\mathrm{CH}_{2}$ & 39.90 & $\begin{array}{c}1.95 \\
-2.06\end{array}$ & 39.90 & $\begin{array}{c}1.95 \\
-2.07\end{array}$ & 39.45 & $\begin{array}{c}1.95 \\
-2.07\end{array}$ & 39.45 & $\begin{array}{r}1.95 \\
-2.07\end{array}$ & 31.37 & $\begin{array}{r}2.04 \\
-2.11\end{array}$ & 31.37 & $\begin{array}{r}2.06 \\
-2.09\end{array}$ & 39.52 & $\begin{array}{c}1.95 \\
-2.02\end{array}$ & 39.52 & $\begin{array}{c}1.96 \\
-2.02\end{array}$ & 31.97 & $\begin{array}{r}1.93 \\
-2.00\end{array}$ \\
\hline 11 & $-\mathrm{CH}_{3}$ & 15.95 & 1.54 & 15.95 & $\begin{array}{r}1.53 \\
-1.54\end{array}$ & 15.96 & 1.55 & 15.96 & $\begin{array}{c}1.54 \\
-1.56\end{array}$ & 23.32 & $\begin{array}{r}1.55 \\
-1.58\end{array}$ & 23.32 & 1.57 & 16.09 & 1.52 & 16.01 & 1.61 & 23.25 & 1.61 \\
\hline 10 & $-\mathrm{C}$ & 134.65 & - & 134.65 & - & 135.29 & - & 135.29 & - & 135.07 & - & 135.07 & - & 134.88 & - & 135.54 & - & 135.83 & - \\
\hline 14 & $-\mathrm{CH}$ & 124.50 & $\begin{array}{c}5.12 \\
-5.18\end{array}$ & 124.50 & $\begin{array}{c}5.12 \\
-5.17\end{array}$ & 123.62 & $\begin{array}{c}5.07 \\
-5.13\end{array}$ & 123.62 & $\begin{array}{r}5.07 \\
-5.13\end{array}$ & 124.26 & $\begin{array}{c}5.02 \\
-5.09\end{array}$ & 124.26 & $\begin{array}{c}5.02 \\
-5.09\end{array}$ & 124.50 & $\begin{array}{r}5.30 \\
-5.35\end{array}$ & 123.62 & $\begin{array}{c}5.03 \\
-5.09\end{array}$ & 124.26 & $\begin{array}{r}5.02 \\
-5.09\end{array}$ \\
\hline 15 & $-\mathrm{CH}_{2}$ & 28.40 & $\begin{array}{r}2.04 \\
-2.08\end{array}$ & 28.40 & $\begin{array}{r}2.02 \\
-2.10\end{array}$ & 26.39 & $\begin{array}{r}1.96 \\
-2.04\end{array}$ & 26.39 & $\begin{array}{c}1.97 \\
-2.04\end{array}$ & 27.89 & $\begin{array}{c}1.77 \\
-1.82\end{array}$ & 27.89 & 1.79 & 28.70 & $\begin{array}{c}2.12 \\
-2.14\end{array}$ & 26.39 & $\begin{array}{r}1.96 \\
-2.04\end{array}$ & 27.89 & $\begin{array}{r}1.78 \\
-1.82\end{array}$ \\
\hline & & & & & & & & & & & & & & Tra & & & & C & \\
\hline 16 & $-\mathrm{CH}_{2}$ & 28.40 & $\begin{array}{r}2.04 \\
-2.08\end{array}$ & 28.70 & $\begin{array}{c}2.10 \\
-2.17\end{array}$ & 25.66 & $\begin{array}{c}1.92 \\
-2.01\end{array}$ & 25.66 & $\begin{array}{r}1.92 \\
-2.01\end{array}$ & 27.89 & $\begin{array}{c}1.77 \\
-1.82\end{array}$ & 27.89 & 1.79 & 28.70 & $\begin{array}{c}2.12 \\
-2.14\end{array}$ & 25.66 & $\begin{array}{c}1.95 \\
-2.01\end{array}$ & 27.89 & $\begin{array}{r}1.78 \\
-1.82\end{array}$ \\
\hline 17 & $-\mathrm{CH}$ & 124.50 & $\begin{array}{r}5.12 \\
-5.18\end{array}$ & 124.50 & $\begin{array}{r}5.30 \\
-5.36\end{array}$ & 123.81 & $\begin{array}{c}5.02 \\
-5.08\end{array}$ & 123.81 & $\begin{array}{r}5.02 \\
-5.08\end{array}$ & 124.26 & $\begin{array}{r}5.02 \\
-5.09\end{array}$ & 124.26 & $\begin{array}{r}5.02 \\
-5.08\end{array}$ & 124.50 & $\begin{array}{r}5.30 \\
-5.35\end{array}$ & 123.81 & $\begin{array}{c}5.02 \\
-5.08\end{array}$ & 124.26 & $\begin{array}{r}5.02 \\
-5.09\end{array}$ \\
\hline 18 & $-C$ & 134.65 & - & 134.88 & - & 135.07 & - & 135.83 & - & 135.07 & - & 135.83 & - & 134.88 & - & 135.83 & - & 135.83 & - \\
\hline 28 & $-\mathrm{CH}_{3}$ & 15.95 & 1.54 & 16.09 & $\begin{array}{r}1.52 \\
-1.53\end{array}$ & 23.32 & $\begin{array}{r}1.56 \\
-1.58\end{array}$ & 23.25 & 1.61 & 23.32 & $\begin{array}{c}1.55 \\
-1.58\end{array}$ & 23.25 & 1.61 & 16.09 & 1.52 & 23.25 & 1.61 & 23.25 & 1.61 \\
\hline 19 & $-\mathrm{CH}_{2}$ & 39.90 & $\begin{array}{c}1.95 \\
-2.06\end{array}$ & 39.52 & $\begin{array}{c}1.97 \\
-2.02\end{array}$ & 31.37 & $\begin{array}{c}2.04 \\
-2.11\end{array}$ & 31.97 & $\begin{array}{c}1.94 \\
-2.02\end{array}$ & 31.37 & $\begin{array}{c}2.04 \\
-2.11\end{array}$ & 31.97 & 1.97 & 39.52 & $\begin{array}{c}1.95 \\
-2.02\end{array}$ & 31.97 & $\begin{array}{c}1.94 \\
-2.02\end{array}$ & 31.97 & $\begin{array}{r}1.93 \\
-2.00\end{array}$ \\
\hline & & & & & & Tra & & & & & & & & & & & & Ci & \\
\hline 20 & $-\mathrm{CH}_{2}$ & 26.90 & $\begin{array}{r}2.05 \\
-2.11\end{array}$ & 26.12 & $\begin{array}{c}2.00 \\
-2.08\end{array}$ & 26.35 & $\begin{array}{c}2.04 \\
-2.12\end{array}$ & 26.76 & $\begin{array}{r}1.93 \\
-1.99\end{array}$ & 26.35 & $\begin{array}{r}2.04 \\
-2.12\end{array}$ & 26.76 & 1.97 & 26.12 & $\begin{array}{c}2.01 \\
-2.06\end{array}$ & 26.76 & $\begin{array}{c}1.94 \\
-2.00\end{array}$ & 26.76 & $\begin{array}{r}1.94 \\
-2.00\end{array}$ \\
\hline 21 & $-\mathrm{CH}$ & 124.60 & $\begin{array}{r}5.09 \\
-5.15\end{array}$ & 124.82 & $\begin{array}{r}5.04 \\
-5.10\end{array}$ & 124.32 & $\begin{array}{c}5.02 \\
-5.07\end{array}$ & 124.82 & $\begin{array}{r}5.07 \\
-5.13\end{array}$ & 124.32 & $\begin{array}{c}5.02 \\
-5.07\end{array}$ & 124.82 & $\begin{array}{c}5.07 \\
-5.13\end{array}$ & 124.82 & $\begin{array}{c}5.04 \\
-5.10\end{array}$ & 124.82 & $\begin{array}{c}5.07 \\
-5.13\end{array}$ & 124.82 & $\begin{array}{r}5.07 \\
-5.13\end{array}$ \\
\hline 22 & $-C$ & 134.85 & - & 135.05 & - & 135.35 & - & 135.05 & - & 135.35 & - & 135.05 & - & 135.05 & - & 135.05 & - & 135.05 & - \\
\hline 29 & $-\mathrm{CH}_{3}$ & 15.95 & 1.60 & 23.35 & 1.68 & 15.95 & $\begin{array}{c}1.59 \\
-1.61\end{array}$ & 23.35 & 1.68 & 15.95 & $\begin{array}{r}1.59 \\
-1.61\end{array}$ & 23.35 & 1.68 & 23.35 & 1.68 & 23.35 & $\begin{array}{c}1.67 \\
-1.69\end{array}$ & 23.35 & 1.68 \\
\hline 23 & $-\mathrm{CH}_{2}$ & 39.90 & $\begin{array}{r}1.92 \\
-1.99\end{array}$ & 31.92 & $\begin{array}{c}2.03 \\
-2.07 \\
\end{array}$ & 39.90 & $\begin{array}{c}1.92 \\
-1.99\end{array}$ & 31.92 & $\begin{array}{r}2.03 \\
-2.07\end{array}$ & 39.90 & $\begin{array}{r}1.93 \\
-1.99\end{array}$ & 31.92 & 2.05 & 31.92 & $\begin{array}{r}2.03 \\
-2.05\end{array}$ & 31.92 & $\begin{array}{c}2.03 \\
-2.07\end{array}$ & 31.92 & $\begin{array}{r}2.03 \\
-2.07\end{array}$ \\
\hline 24 & $-\mathrm{CH}_{2}$ & 26.90 & $\begin{array}{c}1.92 \\
-1.99\end{array}$ & 27.00 & $\begin{array}{c}2.03 \\
-2.07\end{array}$ & 26.90 & $\begin{array}{c}1.92 \\
-2.00\end{array}$ & 27.00 & $\begin{array}{r}2.03 \\
-2.07\end{array}$ & 26.90 & $\begin{array}{c}1.92 \\
-2.00\end{array}$ & 27.00 & 2.06 & 27.00 & $\begin{array}{c}2.05 \\
-2.06\end{array}$ & 27.00 & $\begin{array}{c}2.03 \\
-2.07\end{array}$ & 27.00 & $\begin{array}{c}2.03 \\
-2.07\end{array}$ \\
\hline 25 & $-\mathrm{CH}$ & 124.70 & $\begin{array}{r}5.07 \\
-5.13\end{array}$ & 124.80 & $\begin{array}{r}5.07 \\
-5.13\end{array}$ & 124.70 & $\begin{array}{c}5.07 \\
-5.13\end{array}$ & 124.80 & $\begin{array}{c}5.07 \\
-5.13\end{array}$ & 124.70 & $\begin{array}{r}5.07 \\
-5.13\end{array}$ & 124.80 & $\begin{array}{c}5.07 \\
-5.13\end{array}$ & 124.80 & $\begin{array}{c}5.07 \\
-5.13\end{array}$ & 124.80 & $\begin{array}{c}5.08 \\
-5.13\end{array}$ & 124.80 & $\begin{array}{r}5.07 \\
-5.13\end{array}$ \\
\hline 26 & $-C$ & 131.03 & - & 130.60 & - & 131.03 & - & 130.60 & - & 131.03 & - & 130.60 & - & 130.60 & - & 130.60 & - & 130.60 & - \\
\hline 30 & $-\mathrm{CH}_{3}$ & 17.65 & 1.61 & 17.65 & 1.61 & 17.65 & 1.61 & 17.65 & 1.61 & 17.65 & 1.61 & 17.65 & 1.61 & 17.65 & 1.61 & 17.65 & 1.61 & 17.65 & 1.61 \\
\hline 27 & $-\mathrm{CH}_{3}$ & 25.72 & 1.68 & 25.72 & 1.68 & 25.72 & 1.68 & 25.72 & 1.68 & 25.72 & 1.68 & 25.72 & 1.68 & 25.72 & 1.68 & 25.72 & 1.68 & 25.72 & 1.68 \\
\hline
\end{tabular}


This calculation reveals that the transformation from trans to cis configuration is characteristically associated with the chemical shift of the methylene carbon atom next to the quaternary carbon atom (carbon 19 and 23 shown in Figure 5-4) from 39 - 40 ppm to 31 - 32 ppm and that of the methyl carbon atom at the quaternary carbon atom (carbon 28 and 29 shown in Figure 5-4) from ca. 16 ppm to $23-24$ ppm. These completely fit in the feature of new arising peaks observed in the sample after heating. The chemical shift of the corresponding proton atom of these carbon atoms were obtained by means of the HSQC spectroscopy as 2.04 ppm (at the carbon 19 and 23) and 1.69 ppm (at the carbon 28 and 29), respectively (Figure 5-4). These values also fit to the calculated values, which can be checked in Table 5-2. Furthermore, in the $\mathrm{HMBC}$ spectrum, the following ${ }^{13} \mathrm{C}-{ }^{1} \mathrm{H}$ correlations can be confirmed; 32 ppm (carbon 19 and 23) and 1.6 - 1.7 ppm (proton at the carbon 28 and 29), 32 ppm (carbon 19 and 23) and 5.0 - 5.1 ppm (proton at the carbon 17 and 21), 23 ppm (carbon 28 and 29) and 2.0 ppm (proton at the carbon atom 19 and 23), 23 ppm (carbon 28 and 29) and $5.0-5.1$ ppm (proton at the carbon 17 and 21) (Figure 5-5). Therefore, it can be concluded that the carbon atom 19 or 23 and the carbon atom 28 or 29 are coupling to the same carbon atom 18 or 22, respectively. Calculation results show that the iomerization of one double bond accompanies with a slight chemical shift at the adjacent trans configuration. This corresponds to the fact that several new arising peaks are situated slightly shifted from the original positions. From the above things, isomerization of some trans double bonds to cis configurations in this system can be concluded.

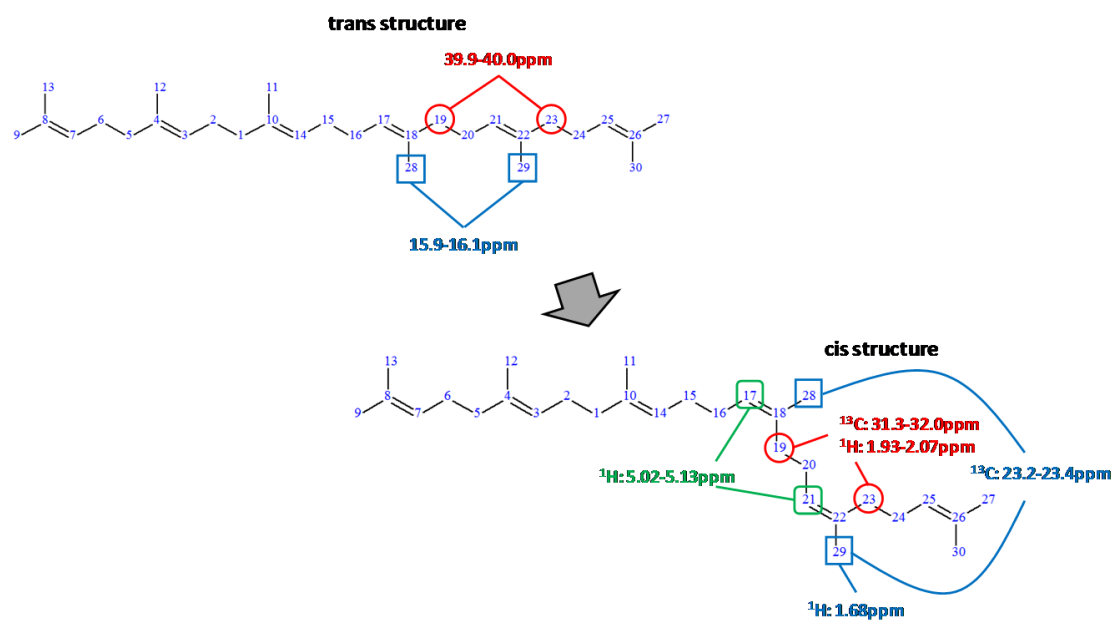

Figure 5-4: Characteristic chemical shift changes in the ${ }^{1} \mathrm{H}$ and ${ }^{13} \mathrm{C}$ NMR spectra between trans and cis structures 


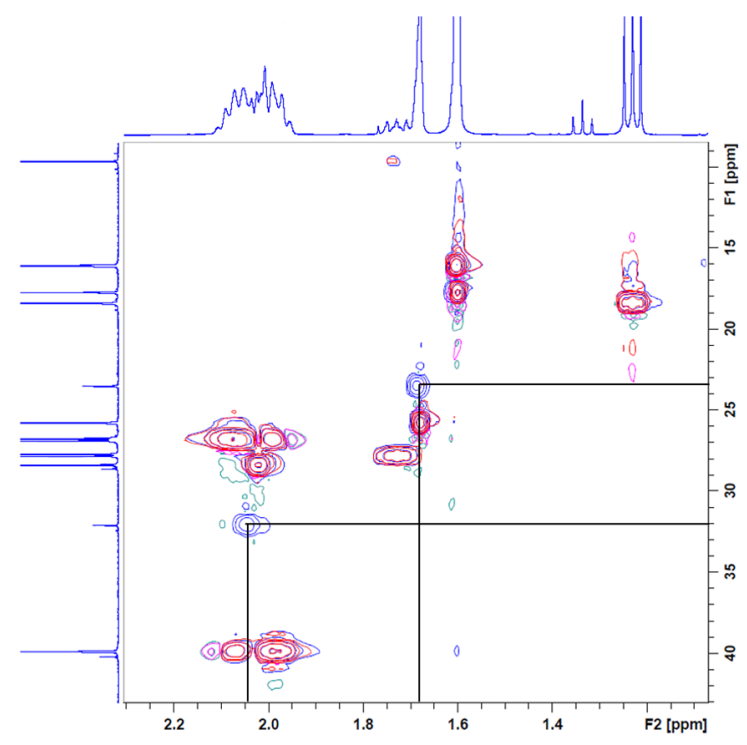

Figure 5-5: HSQC spectra of the squalene / Si 263 mixture (red) before and (blue) after heating at $170{ }^{\circ} \mathrm{C}$ for 60 minutes

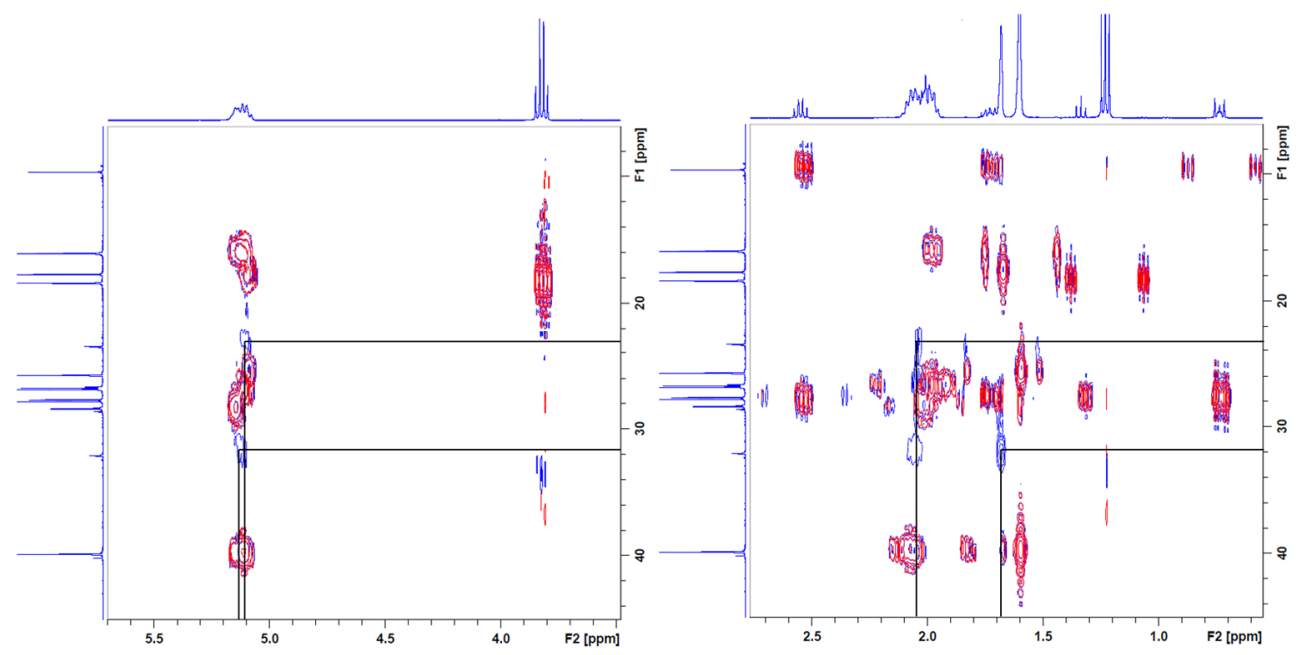

Figure 5-6: $\mathrm{HMBC}$ spectra of the squalene / Si 263 mixture (red) before and (blue) after heating at $170{ }^{\circ} \mathrm{C}$ for 60 minutes 
The LC/MS results are shown in Figure 5-7. A new peak at 31 minutes of the retention time in both HPLC and ESI+ chromatograms is detected in the sample after heating. The mass chromatogram of this peak is shown in Figure 5-7(d). Main mass values are visible as 666,649 and $603 \mathrm{~m} / \mathrm{z}$, which are likely to be ionized substances of the addition reaction product of one Si 263 and one squalene molecule. $649 \mathrm{~m} / \mathrm{z}$ could be a hydrogen ion adduct $(238+410+1=649), 666 \mathrm{~m} / \mathrm{z}$ an ammonia ion adduct $(238+410+18=666)$ and $603 \mathrm{a}$ structure which one ethoxy group lost $(238+410-45=603)$ from the addition reaction product, respectively. Since the retention time of pure squalene is 27 minutes, it seems to be reasonable that the addition reaction product which has a higher molecular weight than squalene has a longer retention time than 27 minutes. The reason for not detecting this product in ${ }^{13} \mathrm{C}$ NMR may be due to the low concentration of this product, which was the same as considered already in the $\mathrm{T} 3 \mathrm{M} 3 \mathrm{H}$ system.

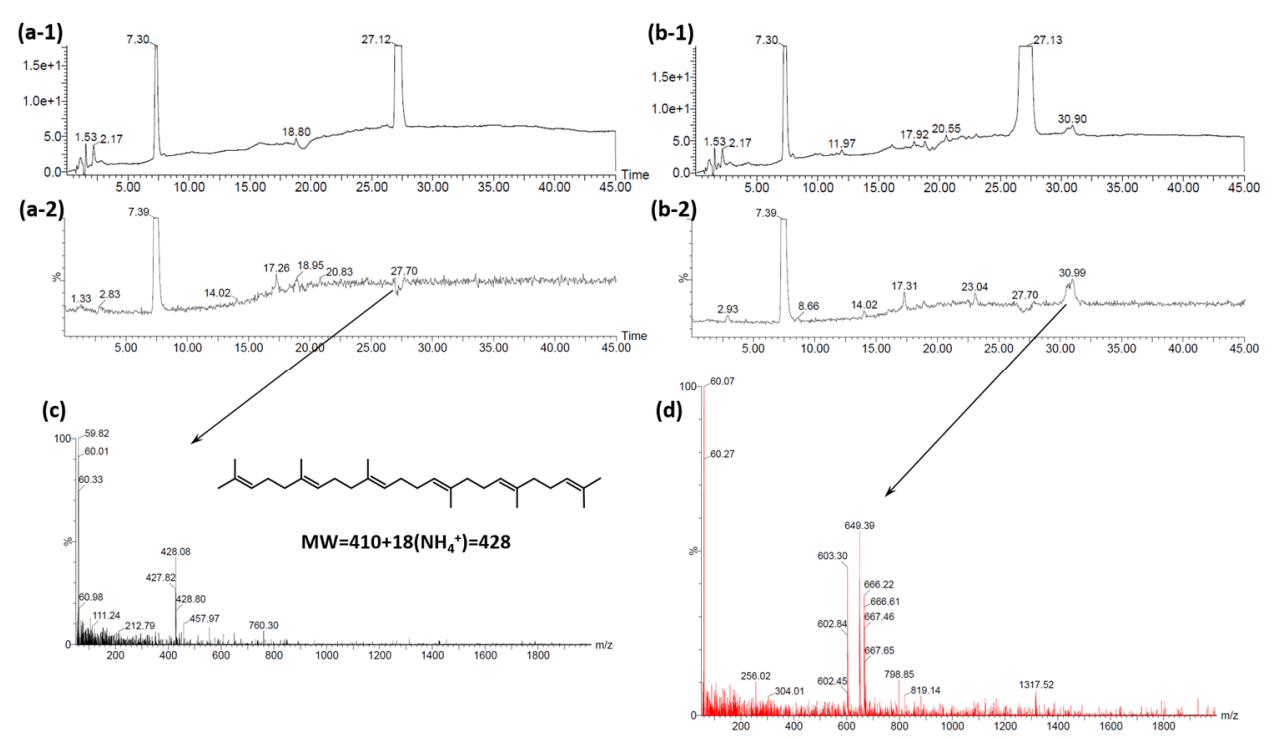

Figure 5-7: LC/MS results of the squalene / Si 263 mixture before and after heating at 170 ${ }^{\circ} \mathrm{C}$ for 60 minutes; HPLC chromatogram (a-1) before heating and (b-1) after heating, ESI+ chromatogram (a-2) before heating and (b-2) after heating, Mass chromatogram at (c) 27.7 minutes and (d) 31.0 minutes

The isomerization degree of squalene in the Si 263 and Si 69 systems are compared. As mentioned before in this sub-chapter, since the peak of the methyl carbon at the cis double 
bond appears at $23-24$ ppm, by evaluating this peak area, the created cis amount can be calculated.

The calculated cis configuration amount in each sample after heating relative to the total amount of double bonds in the original sample (before heating) is depicted in Figure 5-8. As can be clearly seen, the conversion ratio from trans to cis configuration is higher in the $\mathrm{Si}$ 263 system than that in the Si 69 system, which fits to the result already observed in the T3M3H system. However, when the sum of the double bond peak areas are compared considering that squalene originally has peaks at $123.4-125.6 \mathrm{ppm}, 130.6-131.4 \mathrm{ppm}, 134.1$ - 135.6ppm, a different tendency is observed, as shown in Figure 5-9. On one hand the total double bond peak area does not change significantly after heating in all Si 263 samples, on the other hand the value decreases of approximately 7 and $15 \%$ by heating at 150 and 170 ${ }^{\circ} \mathrm{C}$ for 60 minutes respectively in the Si 69 samples.

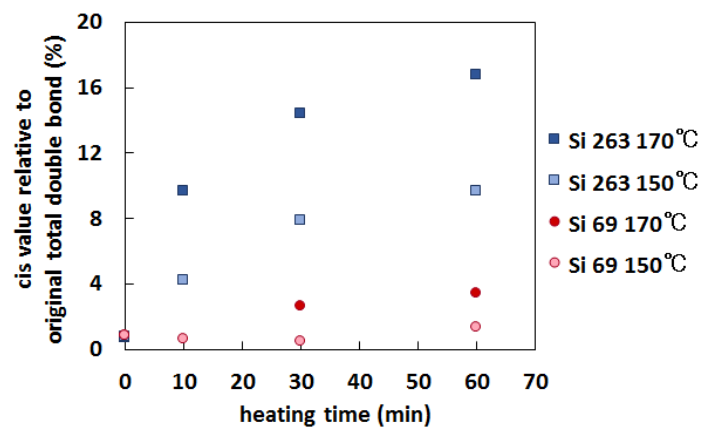

Figure 5-8: Cis configuration ratio relative to the original total double bond peak area in ${ }^{13} \mathrm{C}$ NMR spectra

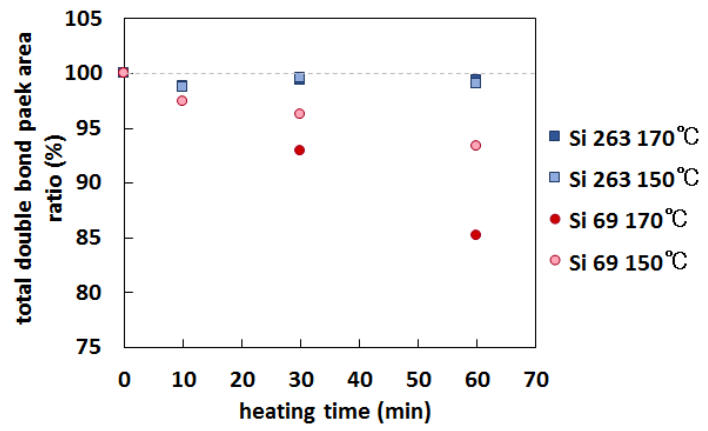

Figure 5-9: Total double bond peak area ratio in ${ }^{13} \mathrm{C}$ NMR spectra 
Actually, many new peaks which were not observed in the Si 263 system are visible now in the Si 69 sample after heating, as can be seen in Figure 5-10 (a). Due to the high amount of signals and also the low intensity of them, it was not possible to identify the exact structures. However, by comparing the ${ }^{13} \mathrm{C}$ NMR spectrum of the Si 69 sample with the one of the squalene / sulfur mixture after heating, the peak positions and the shapes of new resulting peaks are very similar in both spectra (Figure 5-10 (a) and (b)). Therefore, it can be assumed that the created reaction products in the Si 69 system have similar structures than those of the squalene / sulfur mixture. This result agrees to the findings of the TME system. As already reported in the literature [6-9] and also suggested in Chapter 3 of this thesis, $\mathrm{Si}$ 69 seems to be able to release active sulfur species thermally, then the sulfur radicals can create sulfur cross-linked products. Furthermore, signals around 111 and 145 ppm seem to be derived from the newly created terminal double bond. Hence, the following structure as shown in Figure 5-11 is proposed to be the most likeliest reaction product. In the nonaccelerated sulfur vulcanization system, the following mechanism has been suggested. Firstly, sulfur radicals abstract allylic hydrogen from rubber molecules. Secondary, the hydrogen abstracted allylic carbon radicals react with other sulfur radicals, then the intermediate sulfur radicals react with the double bond of rubber molecules, resulting in the formation of cross-linked products. Referring this mechanism together with the fact that the terminal double bond is created in the squalene molecule, the reaction scheme shown in Figure 5-12 could be proposed. 

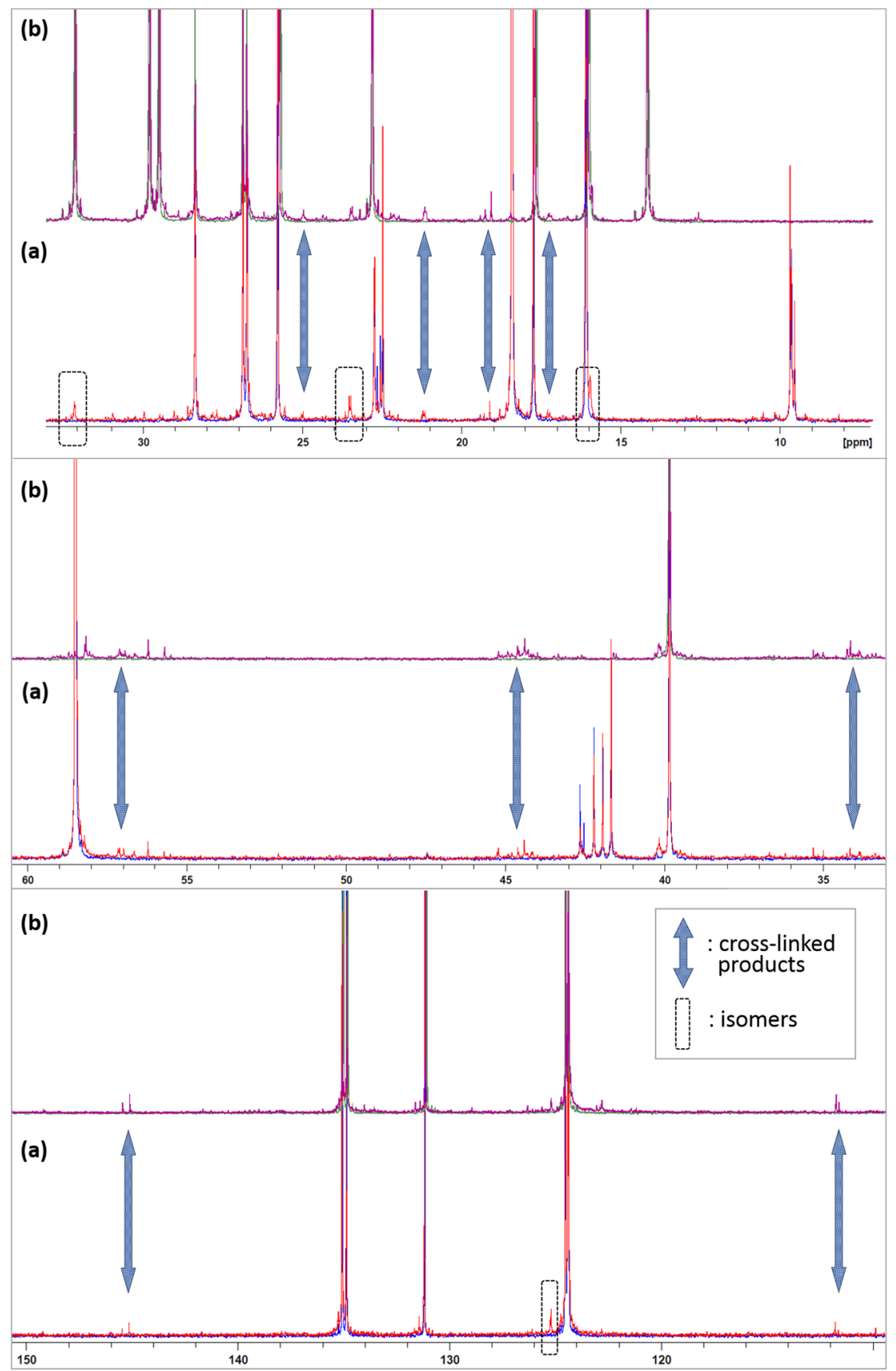

Figure 5-10: ${ }^{13} \mathrm{C}$ NMR spectra of samples (blue) before and (red) after heating at $170{ }^{\circ} \mathrm{C}$ for 60 minutes; (a) squalene / Si 69 mixture, (b) squalene / sulfur / decane mixture 


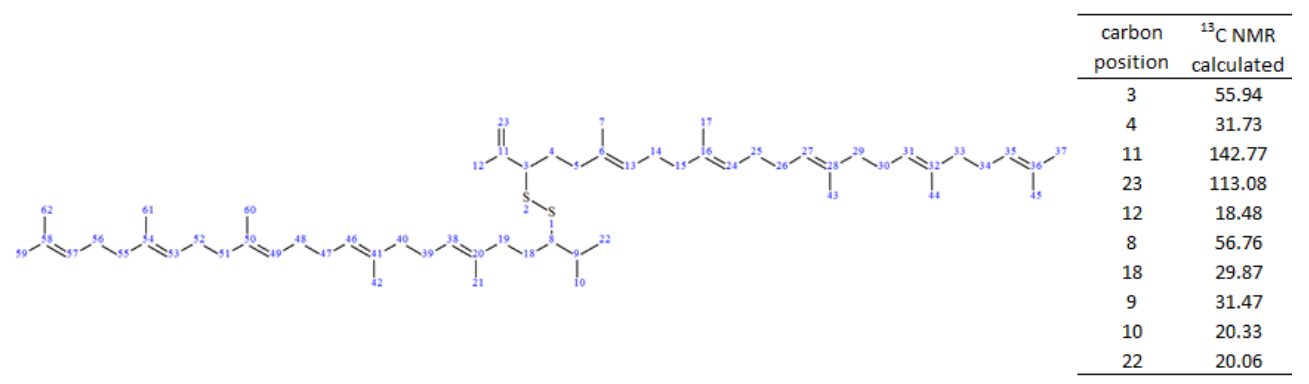

Figure 5-11: Possible generated cross-linked product in squalene / Si 69 mixture

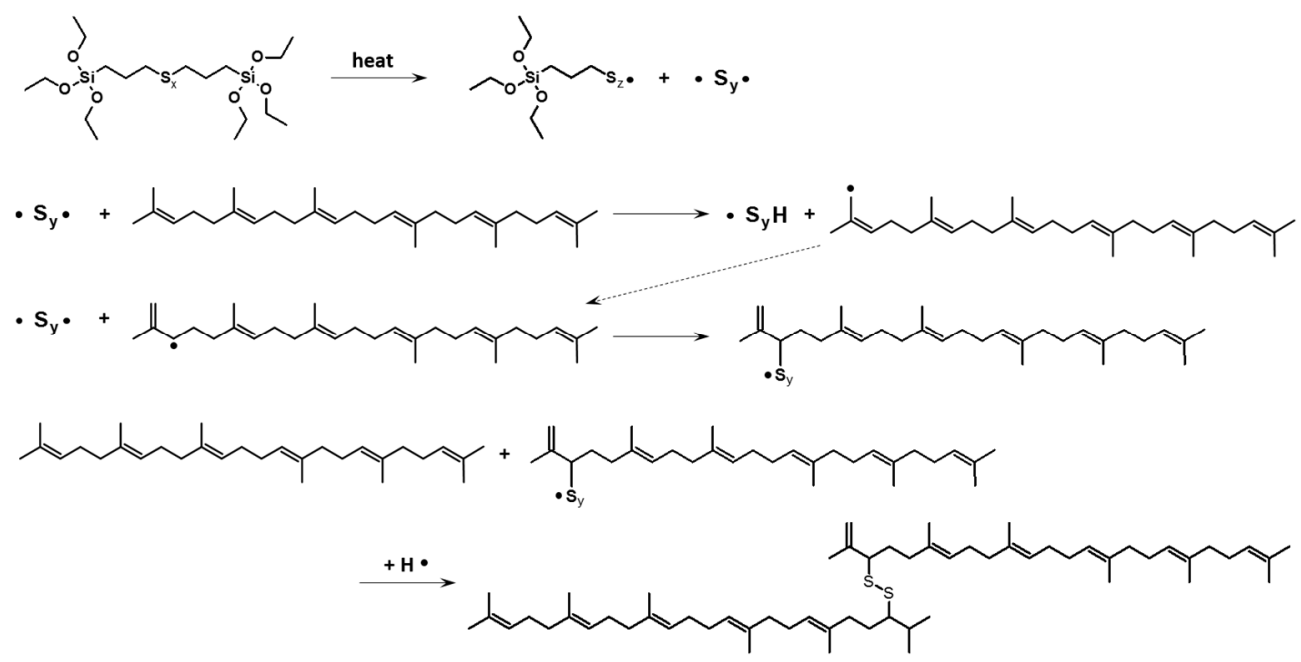

Figure 5-12: Possible reaction scheme in the squalene / Si 69 system

From the results explained so far, it was confirmed that Si 263 and Si 69 follow different reaction mechanisms in the mixture with squalene. In the Si 263 system, a higher isomerization degree from trans to cis configuration was observed compared to the Si 69 system, same as in the model olefin $\mathrm{T} 3 \mathrm{M} 3 \mathrm{H}$ system. And, although the amount seemed to be very small, the addition product of Si 263 to the double bond was able to be detected in the LC/MS measurement. Therefore, it seems to be reasonable to conclude that both of the reversible addition reaction and the allylic hydrogen abstraction by the Si 263 radical have occurred in the system. Higher stability of the original trans configuration of squalene and also the isomerized cis structure in the molecule than the addition reaction structure would be the reason for the low concentration of the remaining addition reaction product. In the Si 69 system, although the isomerization degree was considerably smaller than that in the 
Si 263 system, larger decrease of the total amount of double bonds was observed. This seems to be mainly related to the presence of sulfur active species released from Si 69. And, the direct influence from the dissociate silane component by heating on squalene seemed to be very limited compared to the Si 263 system.

\section{5-3-2 Liquid BR systems}

In the model olefin study, the addition reaction of a mercapto-silane to the double bond, especially with the vinyl and cis double bond, was confirmed to occur. And also, isomerization reaction was observed in both silane systems with different reaction rate. However, those reaction possibilities and rate could be different when using longer molecules because of a higher stearic hindrance effect as well as a different rotation possibility. Therefore, in this section, two liquid BRs with different micro structure were investigated instead of the model olefins in order to gain much closer information for that what can occur in rubber systems.

Figure 5-13 shows the ${ }^{1} \mathrm{H}$ NMR spectra of the Ricon 156 / Si 263 mixture before and after heating at $170^{\circ} \mathrm{C}$ for 60 minutes. After heating, the decrease of the allylic hydrogen (around $2.1 \mathrm{ppm}$ ) and of the vinyl double bond (5.0 and $5.6 \mathrm{ppm}$ ) can be observed as well as an increase of hydrogen (around $2.4 \mathrm{ppm}$ ) at the carbon atom next to the sulfur atom. This is the characteristic pattern from the addition product.

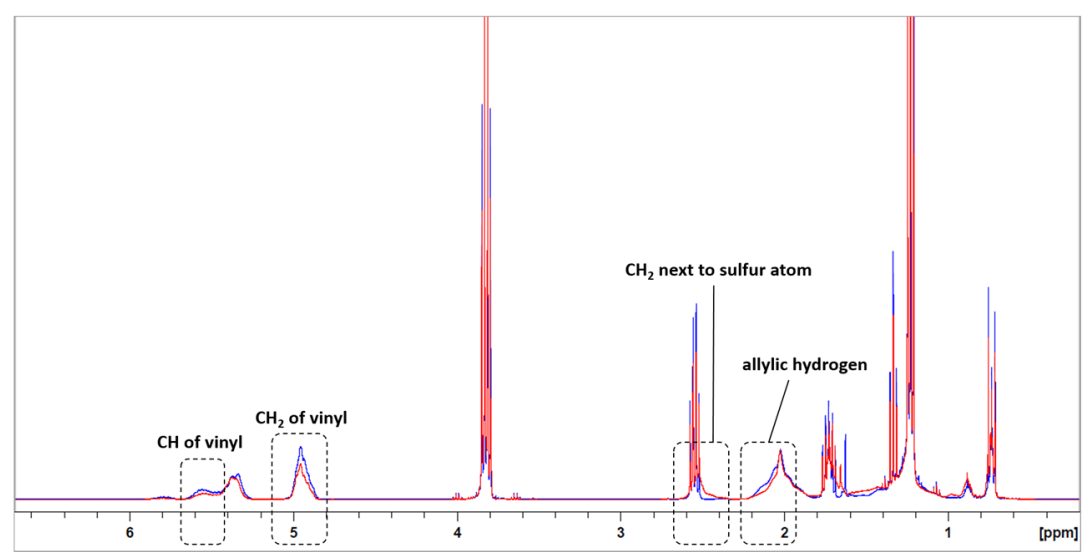

Figure 5-13: ${ }^{1} \mathrm{H}$ NMR of the Ricon 156 / Si 263 mixture (blue) before and (red) after heating at $170^{\circ} \mathrm{C}$ for 60 minutes 
Furthermore, new arising peaks with relatively high intensity at 9.8, 23.2 and 35-36 ppm are visible in the ${ }^{13} \mathrm{C}$ NMR spectrum of the sample after heating, as shown in Figure 5-14. Additionally, the increment of the peak intensity at 29.3, 30.3, 33.0 and 42.5 ppm can be observed. Information of the corresponding hydrogen atoms obtained from the HSQC spectra is summarized in Table 5-3. If the same type of addition reaction of the $\mathrm{Si} 263$ radical to a double bond occur also in the LBR system, the formed structure should have similar chemical shift values as the addition reaction product in the $3 \mathrm{~m} 1 \mathrm{p} / \mathrm{Si} 263$ system at least around the coupling point between a silane and a double bond (around the sulfur atom). Therefore, the chemical shift information obtained in this study is compared to the chemical shifts of the addition reaction product in the $3 \mathrm{~m} 1 \mathrm{p}$ system, as well as to the calculated chemical shift of the structure $(A)$ where a mercapto-silane was added at the end of a vinyl double bond in a butadiene oligomer. 


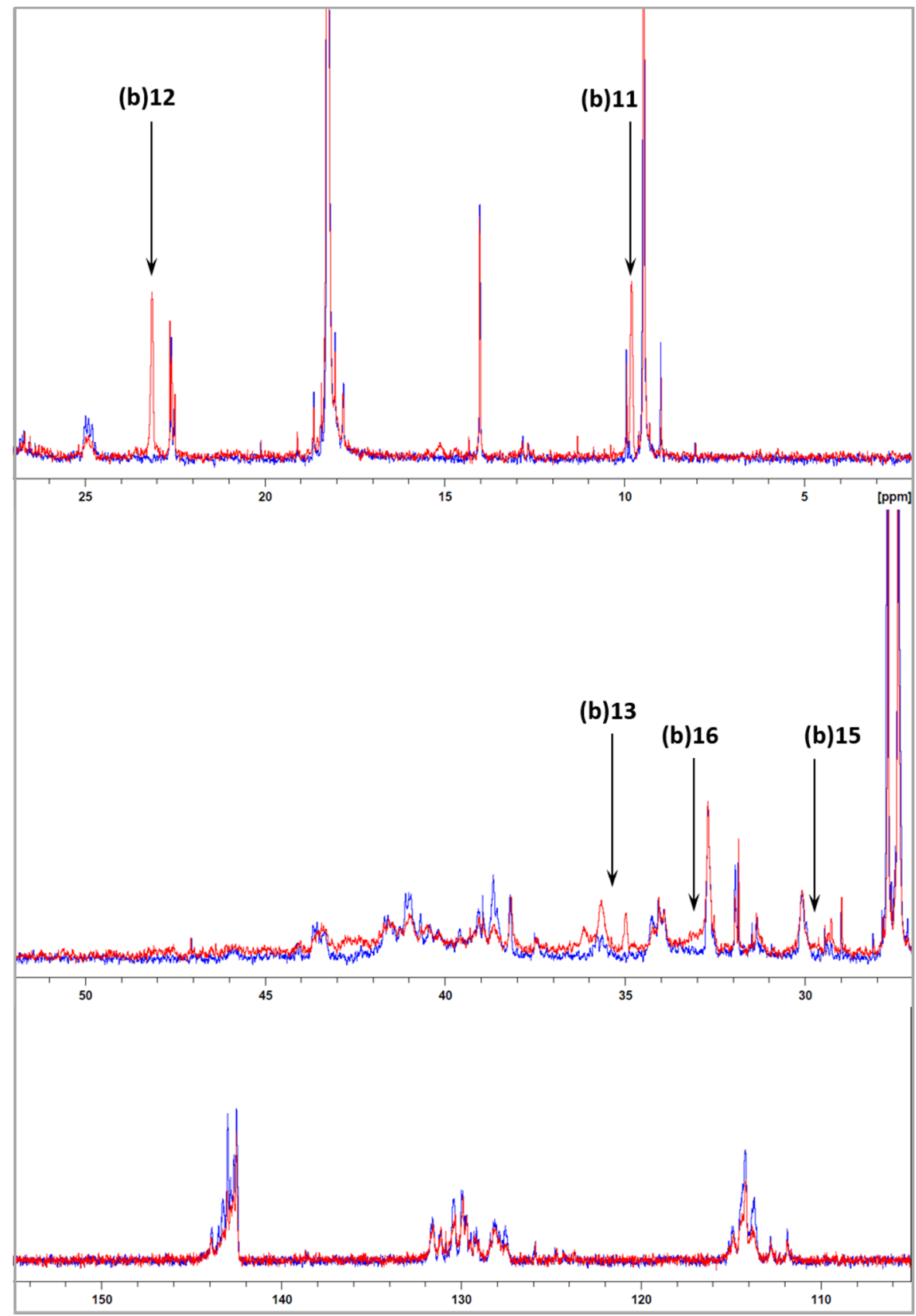

Figure 5-14: ${ }^{13} \mathrm{C}$ NMR of the Ricon 156 / Si 263 mixture (blue) before and (red) after heating at $170{ }^{\circ} \mathrm{C}$ for 60 minutes 
Table 5-3: Chemical shift information of addition reaction products and peak assignments in the NMR spectrum of the Ricon 156 / Si 263 mixture after heating

\begin{tabular}{|c|c|c|c|c|c|c|c|}
\hline \multirow{2}{*}{$\begin{array}{c}\text { number of } \\
\text { carbon atom } \\
\text { in Figures }\end{array}$} & \multicolumn{2}{|c|}{$\begin{array}{l}\text { (a) } 3 \mathrm{~m} 1 \mathrm{p}+\mathrm{Si} 263 \text { addition } \\
\text { product (from chapter } 3 \text { ) }\end{array}$} & \multicolumn{2}{|c|}{$\begin{array}{l}\text { (b) butadiene oligomer } \\
\text { + Si } 263 \text { calculated }\end{array}$} & \multicolumn{3}{|c|}{$\begin{array}{c}\text { Ricon 156+Si } 263 \\
\text { (observed peaks in this study) }\end{array}$} \\
\hline & ${ }^{13} \mathrm{C}$ & ${ }^{1} \mathrm{H}$ & ${ }^{13} \mathrm{C}$ & ${ }^{1} \mathrm{H}$ & ${ }^{13} \mathrm{C}$ & ${ }^{1} \mathrm{H}$ (HSQC) & ${ }^{13} \mathrm{C}$ DEPT \\
\hline 11 & 9.88 & 0.73 & 10.09 & $0.60-0.66$ & 9.81 & 0.73 & $\mathrm{CH}_{2}$ \\
\hline 12 & 23.25 & 1.71 & 24.61 & $1.56-1.67$ & 23.15 & 1.70 & $\mathrm{CH}_{2}$ \\
\hline 13 & 35.03 & 2.53 & 34.34 & $2.40-2.44$ & $34.99,35.67$ & 2.52 & $\mathrm{CH}_{2}$ \\
\hline 15 & 29.61 & 2.50 & 30.98 & $2.47-2.51$ & 29.27 & 2.47 & $\mathrm{CH}_{2}$ \\
\hline 16 & 36.61 & $1.41,1.61$ & 35.42 & $1.39-1.61$ & 33.00 & 1.50 & $\mathrm{CH}_{2}$ \\
\hline 17 & 33.82 & 1.50 & 32.36 & $1.75-1.82$ & 30.30 & 1.72 & $?$ \\
\hline
\end{tabular}

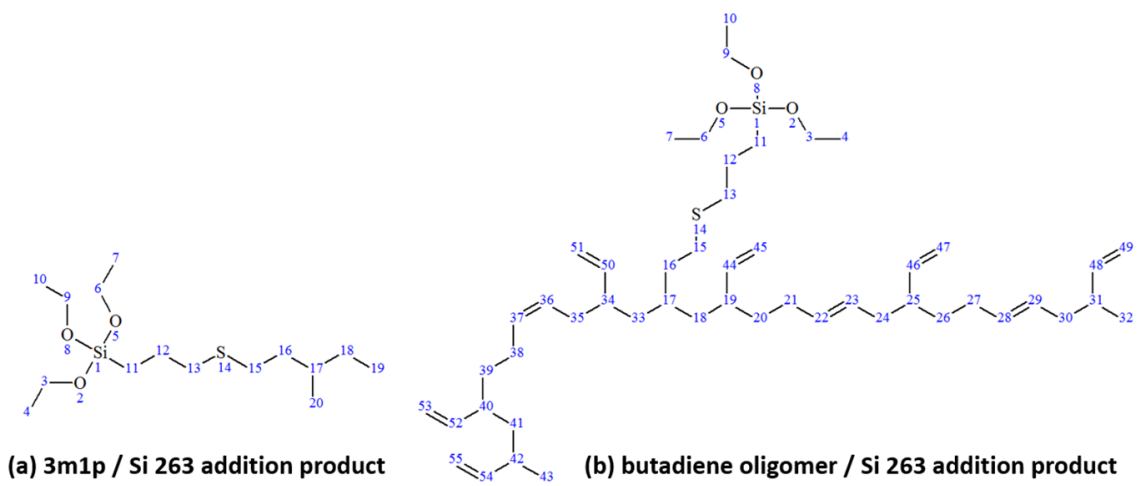

Figure 5-15: (a) Identified addition reaction product in the 3m1p / Si 263 system, (b) possible reaction product between Si 263 and a vinyl double bond in a butadiene oligomer

From the comparison, it can be assumed that the three peaks with high intensity at 9.8, 23.2 and 35 - 36 ppm correspond to the propyl carbon atoms derived from silane added to the end of the vinyl double bond (carbon No. 11, 12, 13 in Figure 5-15(b)). These chemical shift values fit well to those of the addition reaction product observed in the 3m1p / Si 263 mixture. And also, it is confirmed that they are situated in the order from 11, 12 and 13 (Figure 5-16) by means of the combination of the HSQC, COSY and HMBC spectra. Moreover, the corresponding hydrogen peak of the 29.3 ppm carbon atom is confirmed by the HSQC spectrum to be the one at $2.47 \mathrm{ppm}$, hence this carbon atom could be judged as being situated next to the single sulfur atom, which is derived from the end of the vinyl double bond (carbon No. 15 in Figure 5-14(b)). The carbon atom No. 16 which was originally part of the vinyl double bond seems to cause the signal at $33.0 \mathrm{ppm}$. This is further proved by 
the HSQC spectra which shows that the hydrogen atom at the carbon has a chemical shift at $1.50 \mathrm{ppm}$. Also the HMBC spectrum demonstrates that this carbon No. 16 at $33.0 \mathrm{ppm}$ has a correlation with the $2.47 \mathrm{ppm}$ hydrogen atom. All these findings lead to the conclusion that the addition reaction of Si 263 at the end of vinyl double bond in the liquid BR has occurred.
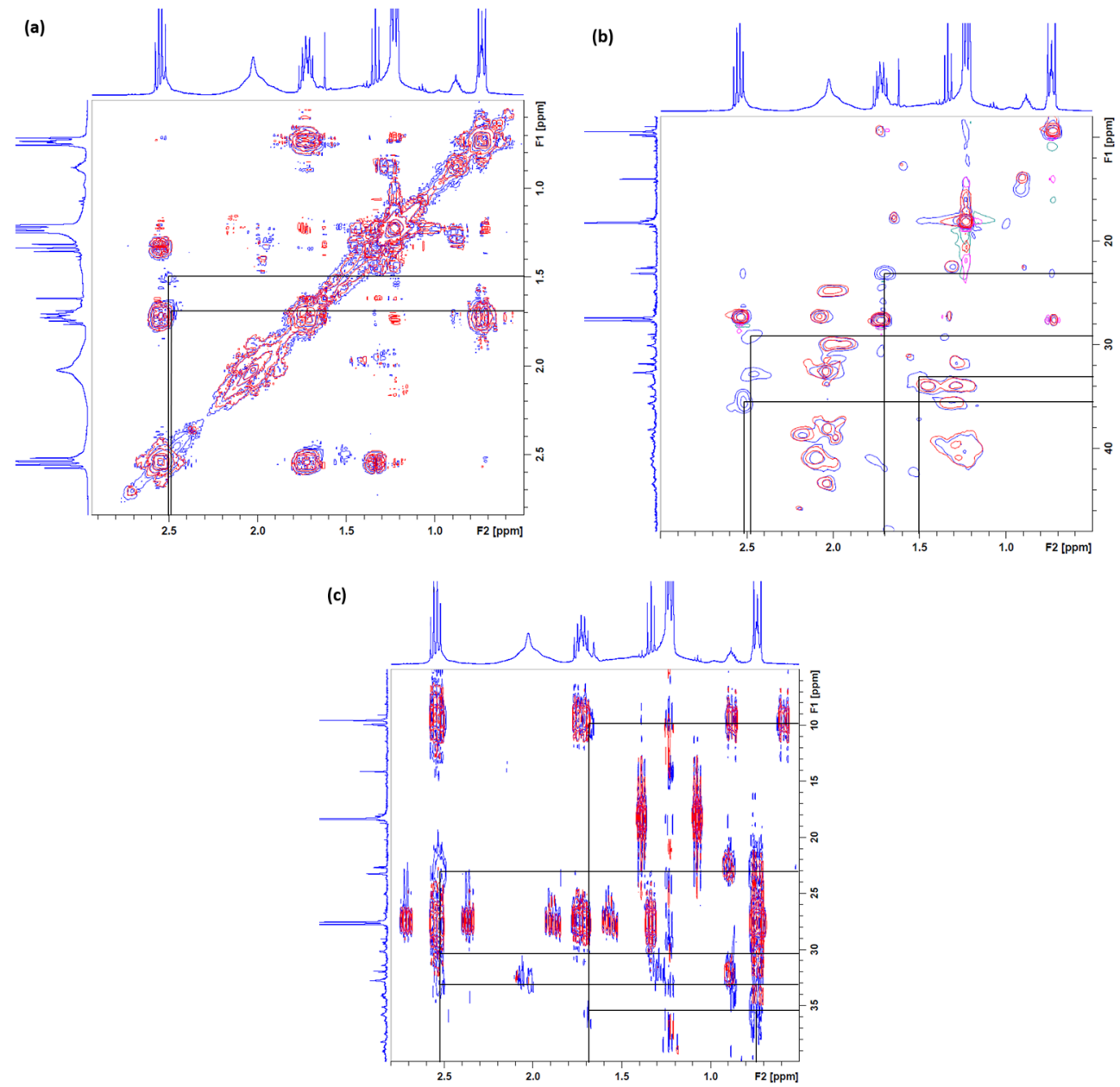

Figure 5-16: 2D-NMR spectra of the Ricon 156 / Si 263 mixture (red) before heating and (blue) after heating for 60 minutes at $170^{\circ} \mathrm{C}$; (a) COSY, (b) HSQC and (c) HMBC 


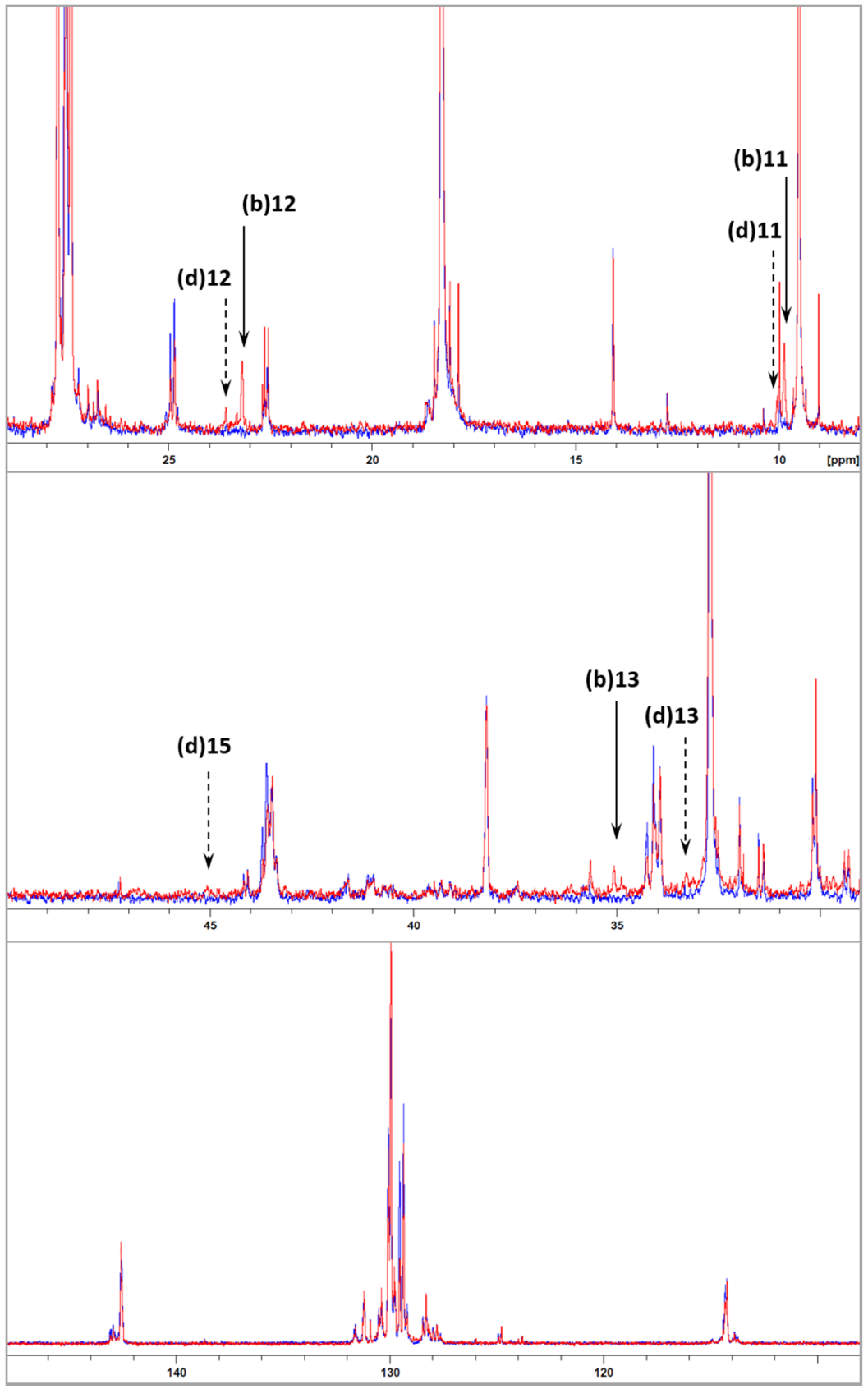

Figure 5-17: ${ }^{13} \mathrm{C}$ NMR of the Ricon 130 / Si 263 mixture (blue) before and (red) after heating at $170{ }^{\circ} \mathrm{C}$ for 60 minutes 
In the ${ }^{13} \mathrm{C}$ NMR spectrum of the Ricon 130 system, which has a lower vinyl content than Ricon 156, though the intensity is lower, the same new peaks at 9.8, 23.2, 35 - 36 ppm after heating, as already observed for Ricon 156, are visible (Figure 5-17). Therefore, it seems that the structure where Si 263 added to the vinyl double bond is produced, as well. While, as shown in Table 5-4, if the Si 263 adds to a cis double bond, the tertiary carbon from the cis double bond where the mercapto group has added seems to appear at 45 - 47 ppm (carbon No. 15 in Figure 5-18(d)). The hydrogen atom situated at this carbon atom is expected to have a chemical shift around $2.5-2.8 \mathrm{ppm}$.

Table 5-4: Chemical shift information of addition reaction products and peak assignments in the NMR spectrum of the Ricon 130 / Si 263 mixture after heating

\begin{tabular}{|c|c|c|c|c|c|c|c|}
\hline \multirow{2}{*}{$\begin{array}{c}\text { number of } \\
\text { carbon atom } \\
\text { in Figures }\end{array}$} & \multicolumn{2}{|c|}{$\begin{array}{l}\text { (C) } \mathrm{C} 3 \mathrm{H}+\mathrm{Si} 263 \text { addition } \\
\text { product (from chapter } 3 \text { ) }\end{array}$} & \multicolumn{2}{|c|}{$\begin{array}{l}\text { (d) butadiene oligomer } \\
\text { +Si } 263 \text { calculated }\end{array}$} & \multicolumn{3}{|c|}{$\begin{array}{c}\text { Ricon 130+Si } 263 \\
\text { (observed peaks in this study) }\end{array}$} \\
\hline & ${ }^{13} \mathrm{C}$ & ${ }^{1} \mathrm{H}$ & ${ }^{13} \mathrm{C}$ & ${ }^{1} \mathrm{H}$ & ${ }^{13} \mathrm{C}$ & ${ }^{1} \mathrm{H}$ (HSQC) & ${ }^{13} \mathrm{C}$ DEPT \\
\hline 11 & 10.32 & 0.73 & 9.99 & $0.66-0.71$ & 10.04 & 0.73 & $\mathrm{CH}_{2}$ \\
\hline 12 & 23.93 & 1.38 & 24.62 & $1.62-1.73$ & 23.61 & 1.67 & $\mathrm{CH}_{2}$ \\
\hline 13 & 33.76 & 2.51 & 32.61 & 2.49-2.56 & 33.25 & 2.50 & $\mathrm{CH}_{2}$ \\
\hline 15 & 47.47 & 2.51 & 45.17 & $2.70-2.77$ & 45.07 & 2.56 & $\mathrm{CH}$ or $\mathrm{CH}_{3}$ \\
\hline 16 & 37.20 & $1.45-1.56$ & 32.91 & $1.22-1.50$ & 34.83 ? & $1.52-1.59$ & $\mathrm{CH}_{2}$ \\
\hline
\end{tabular}

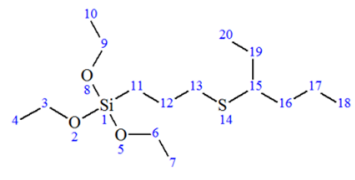

(c) $\mathrm{C} 3 \mathrm{H} / \mathrm{Si} 263$ addition product

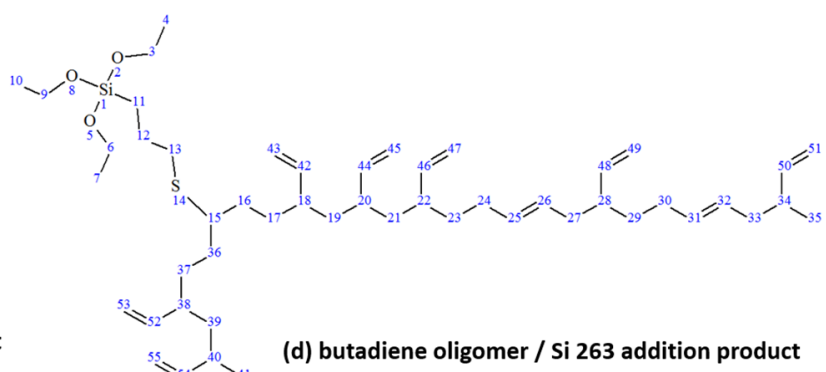

Figure 5-18: (c) Addition reaction product in the C3H / Si 263 system, (d) possible reaction structure between Si 263 and cis double bond in a butadiene oligomer

The HSQC spectrum shows the correlation between the $45.1 \mathrm{ppm}$ carbon atom and the $2.55 \mathrm{ppm}$ hydrogen atom (Figure 5-19). From the incidence of the addition product in the $\mathrm{C} 3 \mathrm{H}$ system and also from the calculated value regarding the addition structure of Si 263 to a cis double bond of a butadiene oligomer, the methylene carbon atom from Si 263 next to 
the sulfur atom (carbon No. 13 in Figure 5-18(d)) is expected to appear at a slightly higher magnetic field compared to the one in the case of a vinyl structure. Hence, there is a high possibility that the carbon atom at $33.25 \mathrm{ppm}$ which has a correlation with the hydrogen atom at $2.5 \mathrm{ppm}$ corresponds to the methylene carbon. Therefore, the addition reaction product of mercapto-silane and the cis double bond seems to be produced here as well, though the concentration seems to be very low.

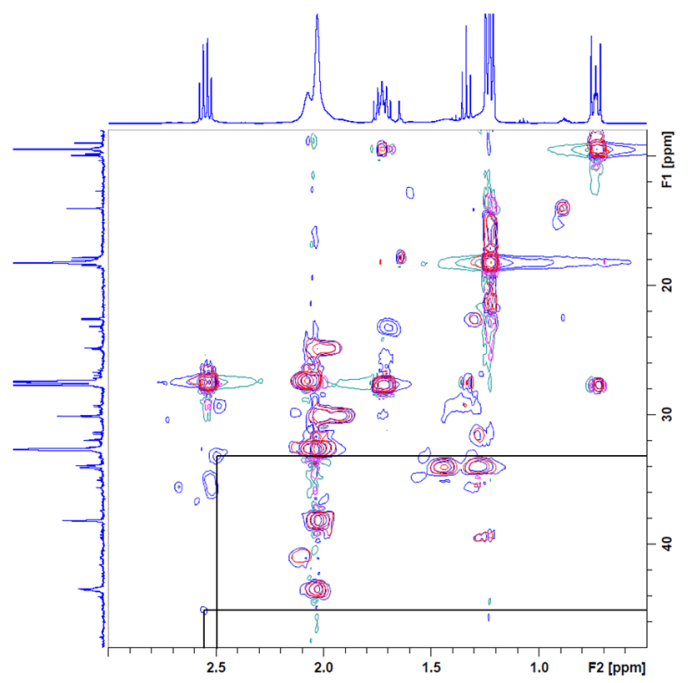

Figure 5-19: HSQC spectrum of the Ricon 130 / Si 263 mixture (red) before heating and (blue) after heating for 60 minutes at $170^{\circ} \mathrm{C}$

In the Si 69 system, the characteristic peaks of the addition reaction structure with one sulfur atom at 9.8, 23.2 and 35 - 36 ppm are not observed in both liquid BRs systems. And also, no indication of another type of addition reaction products (for example, addition reaction product of a dissociated $S_{2}$ part from $\mathrm{Si} 69$ ) was seen in the NMR spectra. Therefore, it can be concluded that the direct reactivity of Si 263 to the double bond of the butadiene polymer is much higher than that in Si 69, and that the reaction of Si 69 is very limited in the absence of further additives like sulfur and accelerator.

Next, the decreased total amount of the remaining cis, trans and vinyl double bonds and the isomerization degree after heating at $170{ }^{\circ} \mathrm{C}$ for 60 minutes were calculated by the combination of the ${ }^{1} \mathrm{H}$ and ${ }^{13} \mathrm{C}$ NMR spectra. Since an internal standard was not added to track the concentration of each component in these systems, the following method was 
used for the calculation. Taking into consideration that the methylene $\left(-\mathrm{CH}_{2}-\right)$ hydrogen atom of the ethoxy group of the silane as well as of the addition reaction product has almost the same chemical shift in the ${ }^{1} \mathrm{H}$ NMR spectrum, the total peak area of this methylene group which is between 3.5 and $4.1 \mathrm{ppm}$ was used as an internal standard. Doing this, it is possible to calculate the vinyl / 1,4 unit (cis + trans) ratio in the ${ }^{1} \mathrm{H}$ NMR using the equation below;

$$
\begin{gathered}
1,4 \text { unit ratio }(\text { mol\% })=(B-A / 2) /(B+A / 2) \\
\text { vinyl unit ratio }(m o l \%)=A /(B+A / 2)
\end{gathered}
$$

where $A$ is the total peak area between 4.8 and $5.0 \mathrm{ppm}$ which corresponds to the peak area of $=\mathrm{CH}_{2}$ in the vinyl structure, $B$ is the total peak area between 5.2 and 5.8 ppm which corresponds to the total peak area of $-\mathrm{CH}=$ in the cis, trans and vinyl structure.

Although it is not possible to calculate the cis / trans ratio by ${ }^{1} \mathrm{H}$ NMR because of an overlapping of these peaks, it becomes possible by using the already reported list of signals in the ${ }^{13} \mathrm{C}$ NMR spectrum, as shown in Table 5-5.

\begin{tabular}{|c|c|c|c|c|c|}
\hline \multirow[b]{2}{*}{ sequence } & \multirow[b]{2}{*}{ carbon $^{a}$} & \multirow[b]{2}{*}{ signal } & \multicolumn{3}{|c|}{ chemical shift, ppm } \\
\hline & & & calcd & obsd & Bywater $^{15}$ \\
\hline C-v & 4 & 1 & $24.52-24.91$ & $24.98-25.10$ & 24.9 \\
\hline C-1,4 & 4 & 2 & 27.44 & $27.42-27.57$ & 27.35 \\
\hline $1,4-\mathrm{C}$ & 1 & & 27.44 & & \\
\hline $\mathrm{T}-\mathrm{v}$ & 4 & 3 & $29.96-30.22$ & 30.16 & 30.1 \\
\hline $\mathrm{v}-\mathrm{v}-\mathrm{C}(\mathrm{m})$ & 1 & 4 & 31.88 & $31.60-32.13$ & 32.1 \\
\hline $1,4-v-C$ & 1 & 5 & 32.67 & 32.72 & 32.65 \\
\hline $\mathrm{T}-1,4$ & 4 & & 32.75 & & \\
\hline $1,4-\mathrm{T}$ & 1 & & 32.75 & & \\
\hline $\mathrm{v}-\mathrm{v}-\mathrm{C}(\mathrm{r})$ & 1 & 6 & 33.39 & $33.35-33.53$ & 33.35 \\
\hline $1,4-\mathrm{V}-1,4$ & 1 & 7 & $34.97-35.08$ & $33.99-34.16$ & 34.15 \\
\hline $1,4-V-v(m)$ & 1 & 8 & $34.37-34.49$ & 34.31 & 34.40 \\
\hline $1,4-V-v(r)$ & 1 & 9 & $35.88-36.00$ & $35.63-36.00$ & 35.75 \\
\hline $\mathrm{v} \cdot \mathrm{v} \cdot \mathrm{T}(\mathrm{m})$ & 1 & 10 & 37.32 & $37.24-37.48$ & 37.4 \\
\hline $1,4-v-T$ & 1 & 11 & 38.26 & 38.18 & 38.1 \\
\hline$v-V-v$ & 2 & 12 & $38.68-39.16$ & $38.57-39.13$ & $\begin{array}{l}38.1 \\
38.6\end{array}$ \\
\hline$v-v-T(r)$ & 1 & 13 & 38.82 & $38.96-39.13$ & \\
\hline $\mathrm{v}-\mathrm{V}$ & 1 & 14 & $39.82-41.33$ & $39.43-41.72$ & $38.9,40.2,41.8^{b}$ \\
\hline $1,4-V-v$ & 2 & 15 & $40.67-41.44$ & $40.55-41.00$ & 40.7 \\
\hline $\mathrm{v}-\mathrm{V}-1,4$ & 2 & 16 & $41.13-41.73$ & $41.00-41.66$ & 41.4 \\
\hline $1,4-V-1,4$ & 2 & 17 & $43.60-44.05$ & $43.47-43.70$ & 43.5 \\
\hline
\end{tabular}

Table 5-5: list of signal of butadiene rubber [10]

Table 5-6 shows the summary of the calculation including the cis / trans ratio. However, since some peaks from silanes and the addition reaction products overlap with those from liquid BRs, the cis / trans ratio may not be so accurate.

$24 \%$ decrease of double bonds is visible in the Ricon 156 (high vinyl content) / Si 263 system, most of them due to a decrease of vinyl double bonds. The amount of cis double 
bonds decreases as well, but as the increase of trans double bonds is seen as well, the cis / trans isomerization can be indicated.

Table 5-6: Concentration change of double bonds

\begin{tabular}{|c|c|c|c|c|c|c|c|c|}
\hline & \multicolumn{8}{|c|}{ Ricon 156} \\
\hline & \multicolumn{4}{|c|}{ Si 263} & \multicolumn{4}{|c|}{ Si 69} \\
\hline & $\begin{array}{c}\text { before } \\
\text { heating }\end{array}$ & & $\begin{array}{c}\text { after } \\
\text { heating }\end{array}$ & difference & $\begin{array}{c}\begin{array}{c}\text { before } \\
\text { heating }\end{array} \\
\end{array}$ & & $\begin{array}{c}\text { after } \\
\text { heating }\end{array}$ & difference \\
\hline Total double bond (\%) & 100 & $\rightarrow$ & 76.4 & -23.6 & 100 & $\rightarrow$ & 89.6 & -10.4 \\
\hline vinyl (mol \%) & 67.7 & $\rightarrow$ & 45.6 & -22.1 & 67.3 & $\rightarrow$ & 60.6 & -6.7 \\
\hline cis (mol \%) & 12.5 & $\rightarrow$ & 8.0 & -4.5 & 12.5 & $\rightarrow$ & 7.2 & -5.3 \\
\hline \multirow[t]{4}{*}{ trans (mol \%) } & 19.8 & $\rightarrow$ & 22.8 & 3.0 & 20.2 & $\rightarrow$ & 21.8 & 1.6 \\
\hline & \multicolumn{8}{|c|}{ Ricon 130} \\
\hline & \multicolumn{4}{|c|}{ Si 263} & \multicolumn{4}{|c|}{ Si 69} \\
\hline & $\begin{array}{l}\text { before } \\
\text { heating }\end{array}$ & & $\begin{array}{c}\text { after } \\
\text { heating }\end{array}$ & difference & $\begin{array}{l}\text { before } \\
\text { heating }\end{array}$ & & $\begin{array}{c}\text { after } \\
\text { heating }\end{array}$ & difference \\
\hline Total double bond (\%) & 100 & $\rightarrow$ & 93.3 & -6.7 & 100 & $\rightarrow$ & 92.2 & -7.8 \\
\hline vinyl (mol \%) & 22.2 & $\rightarrow$ & 18.8 & -3.4 & 22.8 & $\rightarrow$ & 21.1 & -1.7 \\
\hline cis (mol \%) & 35.4 & $\rightarrow$ & 24.7 & -10.7 & 35.6 & $\rightarrow$ & 23.8 & -11.8 \\
\hline trans (mol \%) & 42.4 & $\rightarrow$ & 49.8 & 7.4 & 41.7 & $\rightarrow$ & 47.4 & 5.7 \\
\hline
\end{tabular}

In the Ricon 156 / Si 69 system the decreased amount of total double bonds is only about $10 \%$, which is considerably lower than that in the Si 263 system. The amount of vinyl and cis double bonds decreases similarly, but the rise of the trans double bond is small. This indicates that the cis / trans isomerization in the Si 69 system occurs to a lower degree compared to the Si 263 system.

In the Ricon 130 system, the reduction of the total double bonds is on a comparable level in both silane systems. However, the reduction of the vinyl and the increase of the trans double bonds are higher in the Si 263 system, which is the same tendency as observed in the Ricon 156 systems.

These trends confirm what was expected from the results of the model olefin study. The increased restriction of the molecular rotation because of the longer molecular chain may have led to a decrease of the possibility to undergo an isomerization reaction. However, the main conclusion is still that the addition reaction by silane or sulfur to the double bond and also the generation of polymer radicals by the allylic hydrogen abstraction, which would lead to the isomerization reaction, has occurred in a larger amount in the Si 263 system compared to the Si 69 system. 


\section{5-4 Conclusions}

The reactions of Si 263 or Si 69 with squalene or liquid butadiene rubbers were investigated. The usage of these model substances which have more than one double bond in a molecule and higher molecular weight should simulate the phenomena in the actual tire tread compound more precisely compared to the case by using model olefins.

In the model olefin study, which was described in Chapter 3, on the one hand, a large amount of addition reaction products of mercapto-silane radicals with the vinyl and cis double bond and also a higher degree of isomerization were observed in the Si 263 system compared to the Si 69 system. On the other hand, although the detected amount of addition reaction products and isomerization degree were smaller, a decrease of double bonds could be observed in the Si 69 systems which seems to be because of the influence of active sulfur species released from Si 69.

The phenomena observed in the liquid butadiene and squalene systems were basically consistent with the one observed in the above mentioned model olefin systems. In the liquid butadiene systems, the addition reaction of Si 263 to vinyl and 1,4- cis double bonds was verified. Additionally, a higher degree of cis to trans isomerization was observed compared to that in the Si 69 system. The higher amount of isomerization was detected in the Si 263 model system as well as in the squalene system. The reversible addition reaction of mercapto-silane radicals to the double bonds as well as the allylic hydrogen abstraction accompanied with the radical transfer reaction can be considered as the isomerization mechanisms. As the addition reaction is reversible, the detectable structures in the system after heating should depend on the stability balance between the starting structure, the addition reaction product and isomers, which means that the system should move to the equilibrium condition. In the squalene system, the addition reaction product was observed only in the GC-MS measurement. This can be explained by the fact that the trans and cis double bond structures derived in squalene have a higher stability than the addition reaction product. A large amount of isomerization indicates that large amounts of addition reaction and/or allylic hydrogen abstraction have occurred, which indicates that there is a high possibility that large amounts of carbon radicals in the liquid polybutadiene and squalene molecules can be produced.

In the Si 69 system, the isomerization degree was lower, but the accelerated reduction of a total double bond with increasing heating time was observed. As the ${ }^{13} \mathrm{C} N \mathrm{NM}$ spectrum 
in the Si 69 sample after heating shows a very similar signal pattern as the one observed in the squalene / sulfur system without any accelerators after heating, the formation of crosslinked products also in the Si 69 system is strongly indicated. This corresponds to the result in the model olefin TME system. Therefore, it can be concluded that Si 69 has been acting as a sulfur donor without additional sulfur or accelerators, as already reported in many papers.[6-9]

These different mechanisms between silanes for polymers would be expected to bring big differences in the internal structure including the reinforcing state of the silica filled compounds during a mixing stage. 


\section{5-5 References}

[1] E.C. Gregg, R.P. Lattimer, Rubber Chem. Technol., 57 (1984) 1056-1097

[2] S. Borrós, N. Agulló, Kautsch. Gummi Kunstst., 53 (2000) 131-136

[3] M. Gros, S. Borro's, D.B. Amabilino, J. Veciana, I. Folch, Journal of Mass Spectrometry, 36 (2001) 294-300

[4] E. Vidal-Escales, S. Borrós, Talanta, 62 (2004) 539-547

[5] J. Hahn, M. Runk, M. Schollmeyer, U. Theimer, E. Walter, Kautsch. Gummi Kunstst, 51 (1998) 206-211

[6] S.C. Debnath, R.N. Data, J.W.M. Noordermeer, Rubber Chem. Technol., 76 (2003) 13111328

[7] U. Görl, J. Münzenberg, H.D. Luginsland, A. Müller, Kautsch. Gummi Kunstst., 52 (1999) 588-598

[8] S. Mihara, "Reactive processing of silica-reinforced tire rubber: new insight into the time and temperature-dependence of silica rubber interaction", PhD. Thesis: 2009, Dept. of Elastomer Technology and Engineering, Univ. of Twente, Enschede, the Netherlands.

[9] L.A.E.M Reuvekamp, "Reactive mixing of silica and rubber for tires and engine mounts", Thesis: 2003, Dept. Rubber Technol., Univ. of Twente: Enschede, the Netherlands [10] H. Sato, K. Takebayashi, Y. Tanaka, Macromolecules, 20 (1987) 2418-2423 


\section{Chapter 6}

\section{Rubber compound study-1; \\ Working mechanism of mercapto-silane or sulfide-silane in silica filled SBR/BR compounds}

\section{6-1 Introduction}

In this chapter, the mixing of the typical passenger car tire tread compounds which are SBR / BR blend silica filled compounds in combination with a mercapto-silane or a sulfidesilane is carried out, and the resulting compound properties of two silane systems are compared. The findings in the model study described in the previous chapters will be used in order to explain the obtained different properties in the compounds.

Since there was a difference in terms of the resulting reaction products depending on the double bond structure of the olefins even in the same silane system, especially in the mercapto-silane system, three SBRs having a different micro structure were investigated. For designing the compound, the choice of the polymer is one of the most crucial points, which must be done by considering not only the required tire performance but as well as a sufficient processability. For this point, the influence of the micro structure of the polymer has a strong influence. The best suited micro structure of SBR for the mercapto-silane compounds will be considered from the compound physical properties as well as processability point of view.

In the model study, as one of the main findings, a larger amount of the direct reaction product between a silane and an olefin was obtained in the mercapto-silane system compared to that in the sulfide-silane system, and this reaction product was connected to the silica surface to a high degree as well. Hence, it can be assumed that there should be also a difference regarding the chemically bound rubber content between both silane systems in their rubber compounds. Therefore, in this chapter, the amount of chemically bound rubber will be measured by applying the ammonia treatment. According to the literature, the ammonia treatment can cleave hydrogen bonds and van der waals bonds in 
the bound rubber sample.[1,2] An increasing chemical bound rubber content leads to an extend of the distance between adjacent filler clusters, therefore, it has been reported that this content correlates to the tan $\delta$ value and the Payne effect value in the sulfide-sulane system.[2] In this chapter, the effect of the chemically bound rubber content on the tan $\delta$ and Payne effect values and the mechanism behind it will be discussed by comparing the mercapto-silane and sulfide -silane systems.

Finally, a different reinforcing internal structure of mercapto-silane and sulfide-silane silica filled compounds will be proposed.

\section{6-2 Experimental}

\section{6-2-1 Material and Compound Formulations}

Six different compounds which contain two types of silanes (Si 69 and Si 263) and three types of SBRs having a different micro-structure were prepared. The characteristics of the SBRs used are listed in Table 6-1, the compound formulation in Table 6-2. The added amount of both silanes was fixed as $8 \mathrm{wt} \%$ relative to the used amount of silica. Although $\mathrm{Si}$ 69 has two triethoxy-silyl units in the molecule, Si 263 only one, both silanes have a similar amount of ethoxy groups in the compound by adding $8 \mathrm{wt} \%$ because the molecular weight of Si 69 is nearly twice as high as that of Si 263. Therefore, a similar amount of silanization effect in both silane systems can be expected. In this experiment, no adjustment regarding the net sulfur amount was conducted. Furthermore, since it is expected that Si 263 containing rubber compounds have a faster cure rate as described in chapter 2 , the secondary accelerator DPG was removed from these compounds.

Table 6-1: Specifications of SBRs

\begin{tabular}{|c|c|c|c|c|c|c|c|c|}
\hline & Supplier & $\begin{array}{c}\text { Oil content } \\
{[\mathrm{phr}]}\end{array}$ & $\begin{array}{c}\text { Styrene } \\
(\text { wt.\%) }\end{array}$ & $\begin{array}{c}\text { Cis } \\
(\text { wt.\%) }\end{array}$ & $\begin{array}{c}\text { Trans } \\
\text { (wt.\%) }\end{array}$ & $\begin{array}{c}\text { Vinyl } \\
(\text { wt.\%) }\end{array}$ & $\begin{array}{c}\text { Mooney visc. } \\
\text { ML1+4 [MU] }\end{array}$ & $\begin{array}{c}\text { Tg } \\
\left({ }^{\circ} \mathrm{C}\right)\end{array}$ \\
\hline BUNA VSL5025-2 & Arlanxeo & 37.5 & 28 & 6 & 16 & 50 & 50 & -32 \\
\hline HP755B & JSR & 37.5 & 40 & 13 & 23 & 24 & 65 & -28 \\
\hline SLR6430 & Trinseo & 37.5 & 41 & 16 & 28 & 15 & 72 & -36 \\
\hline
\end{tabular}


Table 6-2: Compound formulation

\begin{tabular}{|l|c|l|}
\hline 1st stage & (phr) & \\
\hline S-SBR & 110 & S-SBR \\
\hline Nipol BR 1220 & 20 & Cis-1,4-BR \\
\hline ULTRASIL 7000 GR & 80 & HD silica \\
\hline Stearic Acid & 2 & Activator \\
\hline Nocrac 6C & 2 & Anti aging agent \\
\hline Si 69 or Si 263 & 6.4 & Silane coupling agent \\
\hline TDAE Oil & 15 & Oil \\
\hline 2nd stage & & \\
\hline ZnO & 2.5 & Activator \\
\hline 3rd stage & & \\
\hline Sulfur & 1.5 & \\
CBS & 1.7 & Accelerator \\
\hline DPG & 2 or 0 & Secondary Accelerator \\
\hline
\end{tabular}

\section{6-2-2 Mixing}

In order to gain understanding of compound property changes depending on the mixing temperature and time, each compound formulation was mixed in the 1st mixing step with six different mixing times according to Table 6-3. All compounds were mixed in total in three steps, the 1st and 2 nd steps are carried out in an $1.6 \mathrm{~L}$ intermeshing type of banbury mixer, the final mixing stage was conducted on an open roll.

Table 6-3: Mixing protocol

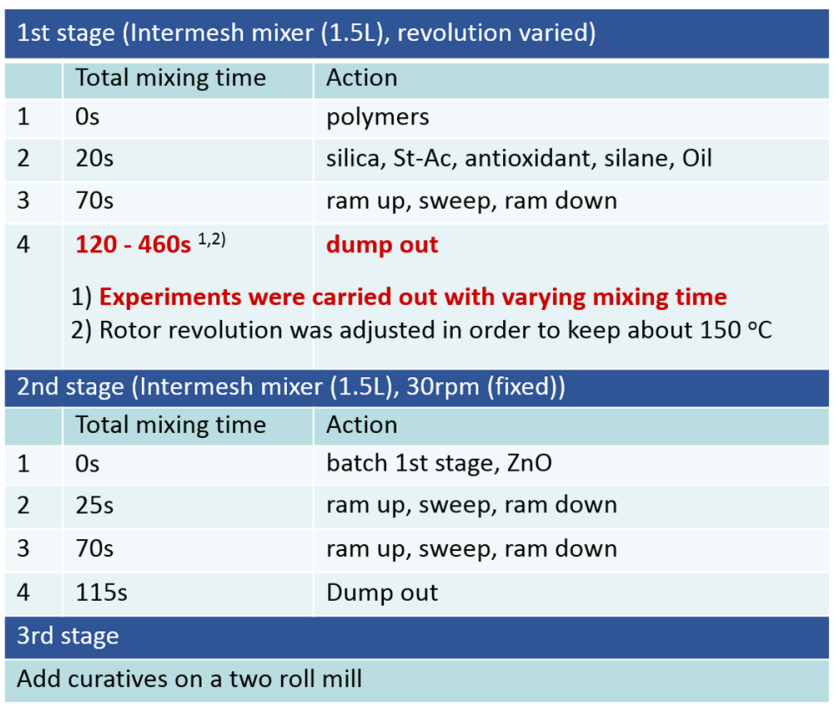




\section{6-2-3 Characterization methods}

The physical properties of these rubber compounds were evaluated as following:

\section{6-2-3-1 Mooney viscosity}

The Mooney viscosity $\mathrm{ML}(1+4)$ measurement of uncured rubber compounds was carried out at $100^{\circ} \mathrm{C}$ by using an L-shaped rotor in a Mooney Viscometer, SMV-300 from Shimadzu. The sample was preheated for 1 minute at $100^{\circ} \mathrm{C}$, and after keeping 4 minutes at the same temperature with a rotor speed of $2 \mathrm{rpm}$, the Mooney viscosity value was measured.

\section{6-2-3-2 Bound rubber measurement}

The bound rubber measurements were carried out both with and without ammonia treatment. The ammonia treatment was performed in order to cleave all hydrogen bonds and van der Waals bonds inside the rubber samples, so that the chemically bound rubber can be determined.[1]. The procedure adopted for the bound rubber measurement was as follows:

- $0.25 \mathrm{~g}$ of compound obtained after 1 st step mixing $\left(W_{b e}\right)$ was placed in a metal cage and immersed in toluene for $72 \mathrm{~h}$ at room temperature. Toluene was renewed every $24 \mathrm{~h}$.

- The sample was removed from the toluene, dried for $72 \mathrm{~h}$ at room temperature and weighed $\left(W_{a f-1}\right)$.

- The sample was immersed again in toluene for $72 \mathrm{~h}$ at room temperature in an ammonia atmosphere in order to cleave the hydrogen bonds and van der Waals bonds. Toluene was renewed every $24 \mathrm{~h}$.

- The sample was dried for $72 \mathrm{~h}$ at room temperature and weighed $\left(W_{a f-2}\right)$.

The overall or chemically bound rubber contents are calculated as a ratio of the amount of the rubber which was insoluble in toluene ( $\fallingdotseq$ the rubber attached to silica) relative to the original amount of the rubber in the sample using the following equation (1):

$$
\text { Bound rubber content (\%) }=\frac{W_{a f-10 \mathrm{r} 2}-W_{f}}{W_{p}} \times 100
$$

where $W_{a f-1}$ and $W_{a f-2}$ are the weight of samples after 1 st extraction and 2 nd extraction with an ammonia, respectively. $W_{f}$ is the weight of silica and $W_{p}$ is the weight of polymer in the original sample (before immersed in toluene), which are calculated by the following equations (2), (3) with reference to the compound formulation and initial sample weight $\left(W_{b e}\right)$ : 


$$
W_{p}=W_{b e} \times \frac{100 p h r}{\text { total phr }} \quad \text { (2), } \quad W_{f}=W_{b e} \times \frac{\text { silica } p h r}{\text { total phr }}
$$

\section{6-2-3-3 Swelling degree of bound rubber by toluene}

In order to obtain information of the structure of the chemical bound rubber, its swelling degree in toluene was measured. The resulting rubber sample after the ammonia treatment, placed in a metal cage was immersed in toluene for $72 \mathrm{~h}$ at room temperature. The sample was taken out from toluene and weighed after wiping out outside the cage to get rid of the outer attached toluene. The swelling degree was calculated by the following equations (4):

$$
\text { Swelling degree }(\%)=\frac{V-V_{0}}{V_{0}} \times 100
$$

where $V_{0}$ and $V$ are the volume of chemical bound rubber samples before and after immersed in toluene, respectively.

\section{6-2-3-4 Payne effect}

Payne effect measurements were carried out by using a Rubber Process Analyzer, RPA2000 from Alpha Technologies, to characterize uncured and cured compounds. The cured compounds were heated for 10 minutes at $170^{\circ} \mathrm{C}$. In order to assess the Payne effect value, the storage moduli was measured from low strain to high strain at $110{ }^{\circ} \mathrm{C}$ and a frequency of $60 \mathrm{cpm}$. The difference of storage moduli at low and high strain was calculated as the Payne effect value for uncured and cured compound by using the following equations, respectively:

$$
\begin{gathered}
\text { Payne effect for uncured compound }(\mathrm{MPa})=G^{\prime}(0.28 \%)-G^{\prime}(450 \%) \\
\text { Payne effect for cured compound }(\mathrm{MPa})=G^{\prime}(0.28 \%)-G^{\prime}(30 \%)
\end{gathered}
$$

\section{6-2-3-5 Flocculation measurement}

In order to investigate the flocculation behavior, the $G^{\prime}$ value at $1 \%$ shear strain was monitored for $10 \mathrm{~min}$ at $130{ }^{\circ} \mathrm{C}$, at a frequency of $60 \mathrm{cpm}$ using a RPA2000.

\section{6-2-3-6 Viscoelasticity (tan $\delta)$}

The $\tan \delta$ at $60^{\circ} \mathrm{C}$ value was measured for vulcanized compounds using a RheolographSolid from Toyo Seiki under the conditions of a frequency of $20 \mathrm{~Hz}$, an initial static strain of $10 \%$ and an amplitude of $\pm 2 \%$. 


\section{6-3 Results and Discussion}

\section{6-3-1 Bound rubber content before and after ammonia treatment}

Figure 6-1 shows the overall bound rubber content of each sample after the 1st mixing step with varying mixing times. As expected, though the bound rubber content increases with increasing mixing time in both silane systems, the bound rubber content inside the compounds with Si 263 shows always higher values. On the one hand the bound rubber content of the Si 263 compounds which were dumped at $120^{\circ} \mathrm{C}$ (leftmost points in Figure $6-1$ ) is already beyond $60 \%$, on the other hand that of the Si 69 compounds is only between 20 and $30 \%$.

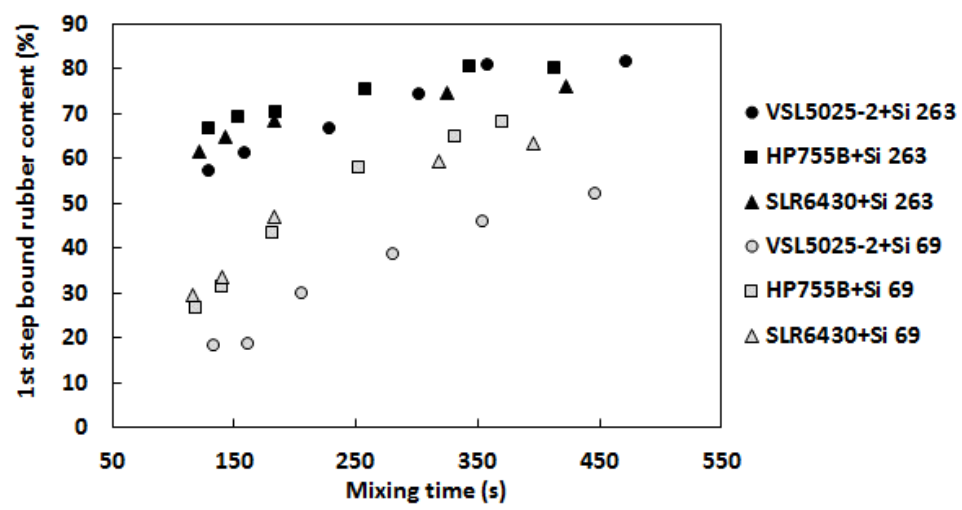

Figure 6-1: Overall bound rubber content of 1st step mixing compounds with different mixing time

In order to remove the physical linked rubber with the silica, an ammonia treatment was applied. Since the hydrogen bonds between silica surfaces are also broken by the treatment, certain amount of occluded rubber could be released as well. The resulting detached and remaining amount of bound rubber contents which are termed as physically and chemically bound rubber respectively are shown in Figure 6-2.[1,2] The bound rubber in the all SBR / Si 263 systems is mostly chemical nature, and the amount of it is significantly higher than that in Si 69 compounds, while the physical bound rubber content is higher in Si 69 compounds. Therefore, the reason for the higher overall bound rubber content in the (short time mixing) Si 263 compounds cannot be the higher physical interaction between the rubber and the silica surface. 


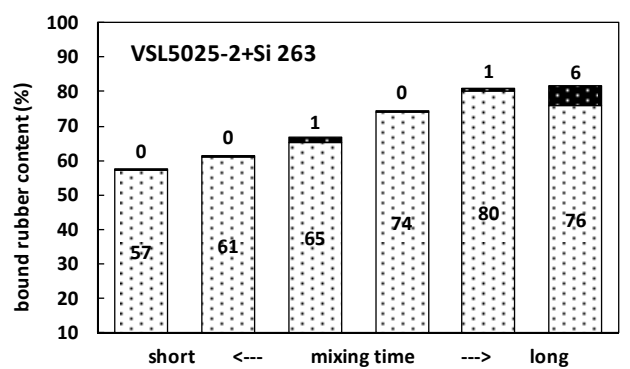

$\square$ Chemically bound rubber (\%) Physically bound rubber (\%)

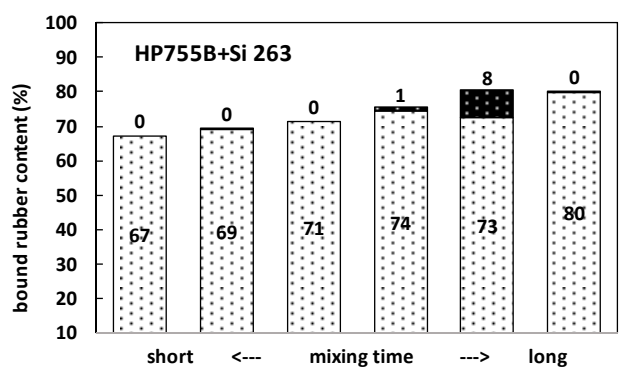

$\square$ Chemically bound rubber (\%) D Physically bound rubber (\%)

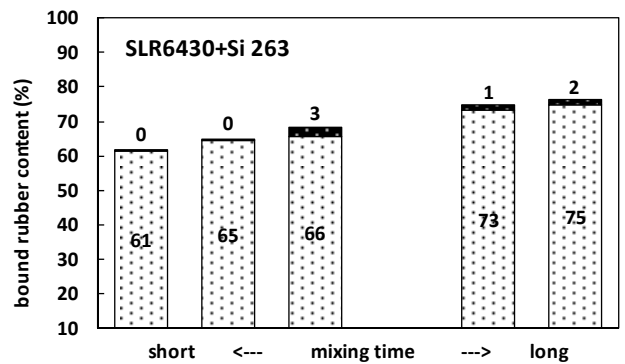

$\square$ Chemically bound rubber (\%) Dhysically bound rubber (\%)

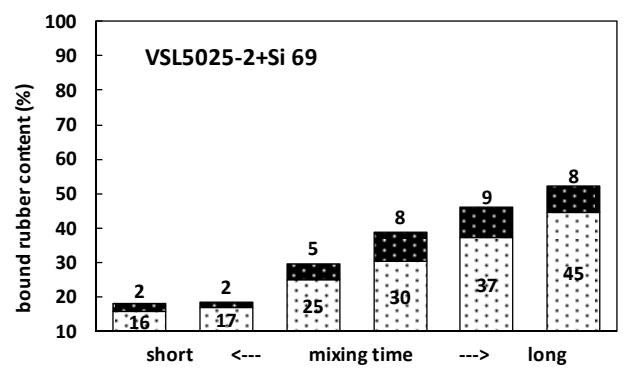

$\square$ Chemically bound rubber (\%) Physically bound rubber (\%)

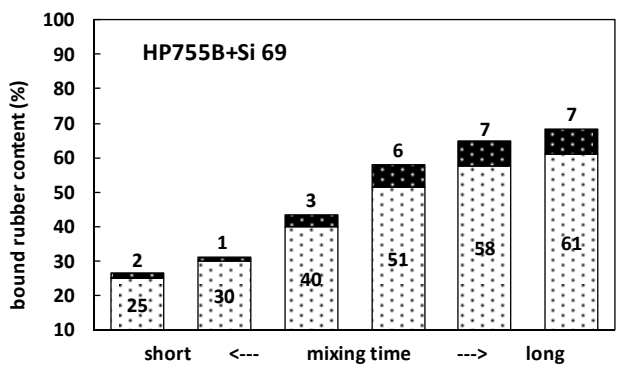

$\square$ Chemically bound rubber (\%) Physically bound rubber (\%)

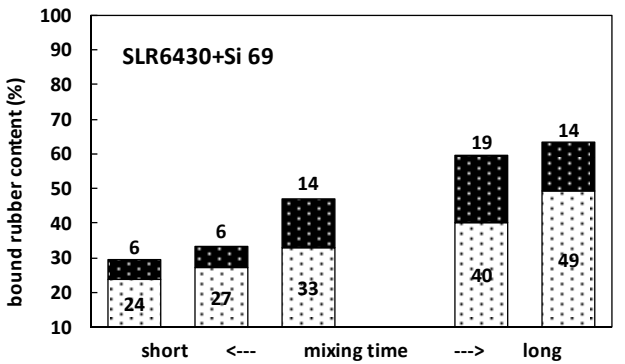

$\square$ Chemically bound rubber (\%) Physically bound rubber (\%)

Figure 6-2: Physically and chemically bound rubber content of 1st step mixing compounds

\section{6-3-2 Swelling degree of chemical bound rubber by Toluene}

The observed facts so far imply that there are differences in terms of the mechanism to create the chemically bound rubber on the silica surface between two silane systems. In order to obtain further information regarding the structure of the chemically bound rubber, the swelling degree by toluene of it was measured (Figure 6-3). The amount of toluene which the bound rubber can absorb increases with increasing bound rubber content, which 
results in a higher degree of swelling. The degree of swelling in Si 263 compounds is considerably lower than that in Si 69 compounds even when compounds with an identical chemically bound rubber content of $60 \%$ are compared. Based on the assumption that the bound rubber in each compound has a homogeneous structure, it can be judged that a significantly denser polymer network has been formed in Si 263 compounds from the initial period of the mixing sequence compared to Si 69 compounds (Figure 6-4).

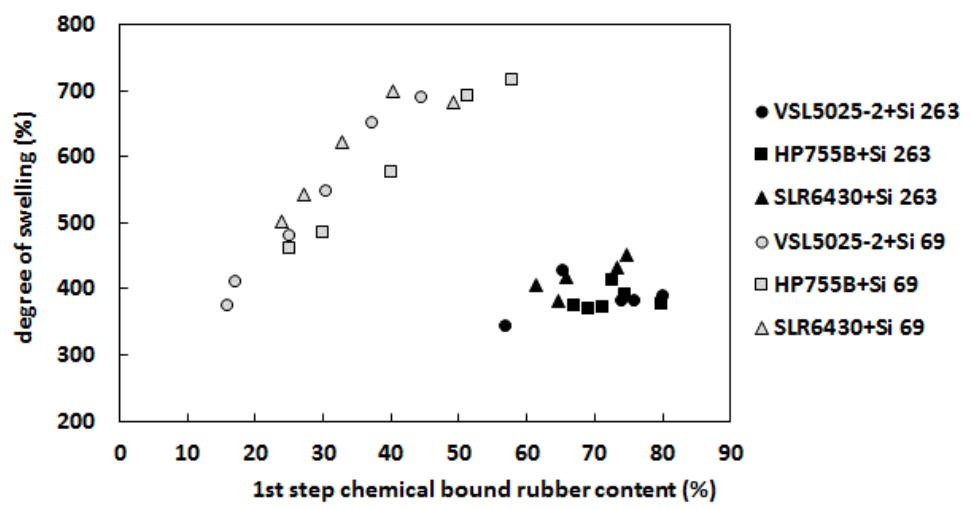

Figure 6-3: Degree of swelling of chemically bound rubber by toluene

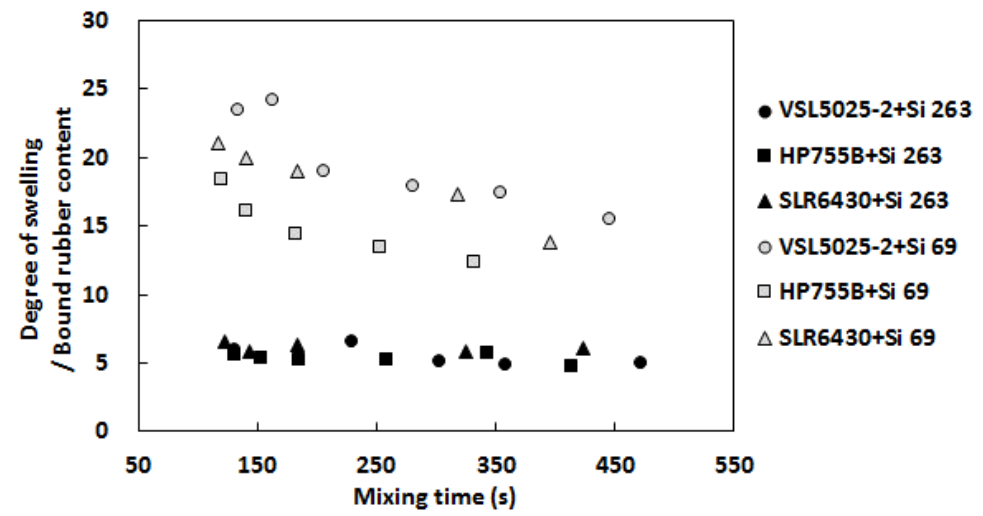

Figure 6-4: Degree of swelling divided by the chemically bound rubber content vs. mixing time

These results can be explained by the characteristics in Si 263 compounds observed in the model study as following. Firstly, since the mercapto-radical can react with the double bond of the rubber directly during the mixing process, especially with the vinyl structure, 
this could raise the possibility to increase amount of the chemically bound rubber drastically. It was confirmed in further experiments that a temperature of $110^{\circ} \mathrm{C}$ is high enough for the addition reaction with the vinyl double bond. Considering a possibility that polymer radicals are generated also due to a polymer main chain scission by the mechanical shear forces during mixing in the rubber compound, it is also supposed that the generation of mercaptoradicals by releasing a hydrogen radical could be accelerated by this process as well. Therefore, the addition reaction could also occur even at lower temperatures than $110{ }^{\circ} \mathrm{C}$. Another point is that Si 263 causes a larger amount of isomerization in the model system compared to Si 69, as described in Chapter 3. The following mechanisms can be considered for the isomerization process. In the process of the reversible addition reaction of the mercapto-silane radical to the double bond, the intermediate (thioether and carbon centered) radical can be isomerized. Furthermore, the olefin or polymer radicals created by the abstraction of the allylic hydrogen by the mercapto-silanes and the above mentioned intermediate carbon centered radicals can be delocalized. This can occur due to the neighbored double bond which results in an isomerization of them. In the literature, it has been proposed that the intermediate carbon radicals which are produced by the addition reaction of the mercapto-silane radical to the vinyl double bond abstract the neighbored intramolecular hydrogen, which results in varying polymer radicals (Figure 6-5).[3] As already concluded in Chapter 3 and 5, a larger generation of polymer radicals can be expected in the mercapto-silane system compared to the sulfide-silane system. Since SBR is generally known to undergo chain transfer, $[4,5]$ it can be expected that SBR starts transferring a generated radical inside the polymer chain or transferring it to another polymer chain, which results in forming a carbon-carbon covalent bond. In fact, it has been reported that in the peroxide vulcanization system, which proceeds in the radical mechanism same as considering now in the mercapto-silane system, one peroxide molecule creates 10 or more crosslinks in polybutadiene system.[6,7] The repetition of this reaction is considered to result in the formation of a denser polymer network and also in an increase in the total amount of bound rubber, as depicted in Figure 6-6. If one part of the polymer network reacts with silica via a silane, the rapid and large generation of the bound rubber in the short mixing time could be explained by the above described mechanism. Furthermore, the dense polymer network which cannot be dissolved in toluene may trap silica clusters like "occluded silica". As a consequence, even in the absence of a direct chemical linkage between the polymer and the silica, the polymer part could be counted as a chemically bound rubber. 


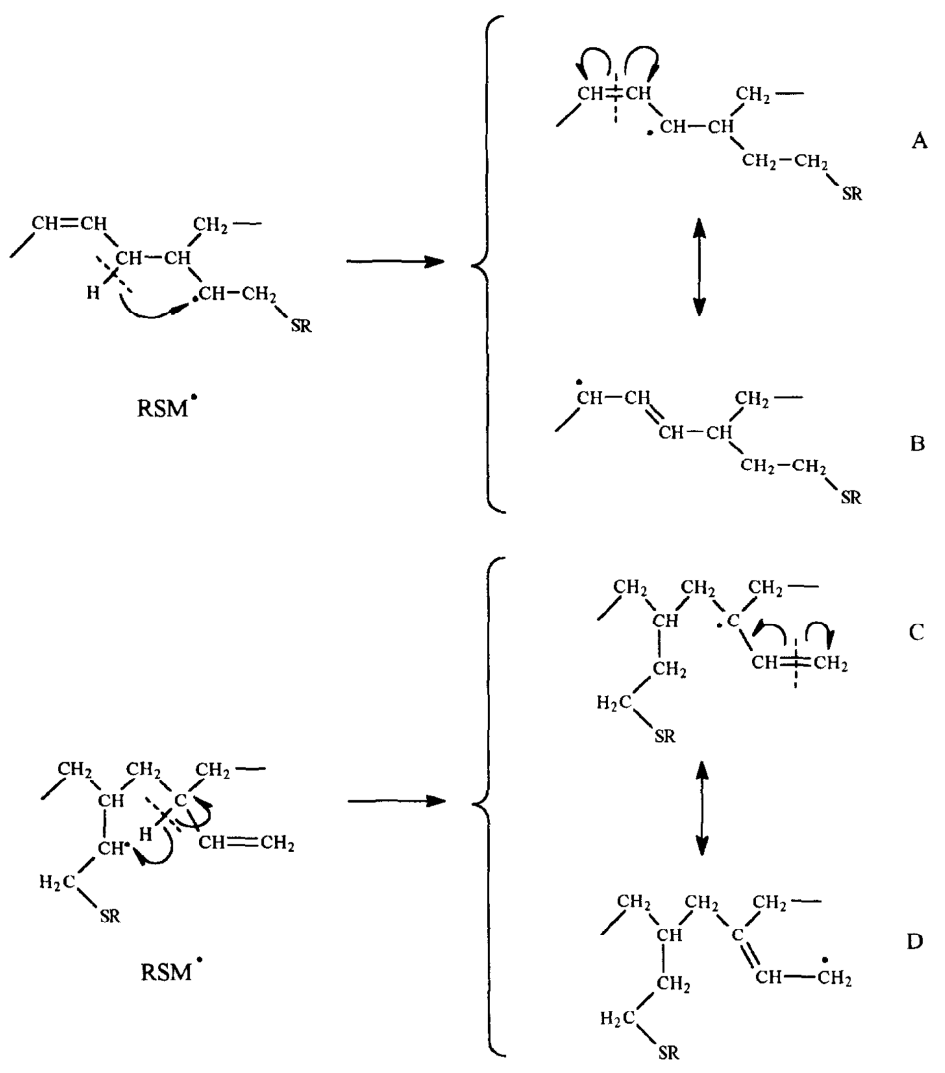

Figure 6-5: Proposed intramolecular rearrangement of the mercapto-silane added carbon radicals [3]

This denser chemically bound rubber structure may give another interpretation for the fact that the physically bound rubber content in the SBR / Si 263 system showed lower value compared to other systems. As mentioned before, it can be supposed that the ammonia treatment cleavages the hydrogen bond between the surface of silica clusters, which leads to release of the occluded rubber. However, the occluded rubber in the SBR / Si 263 system may have been kept trapping due to the denser and thick chemically bound rubber network inside the system, which results in the lower detectable amount of physically bound rubber in the system. 


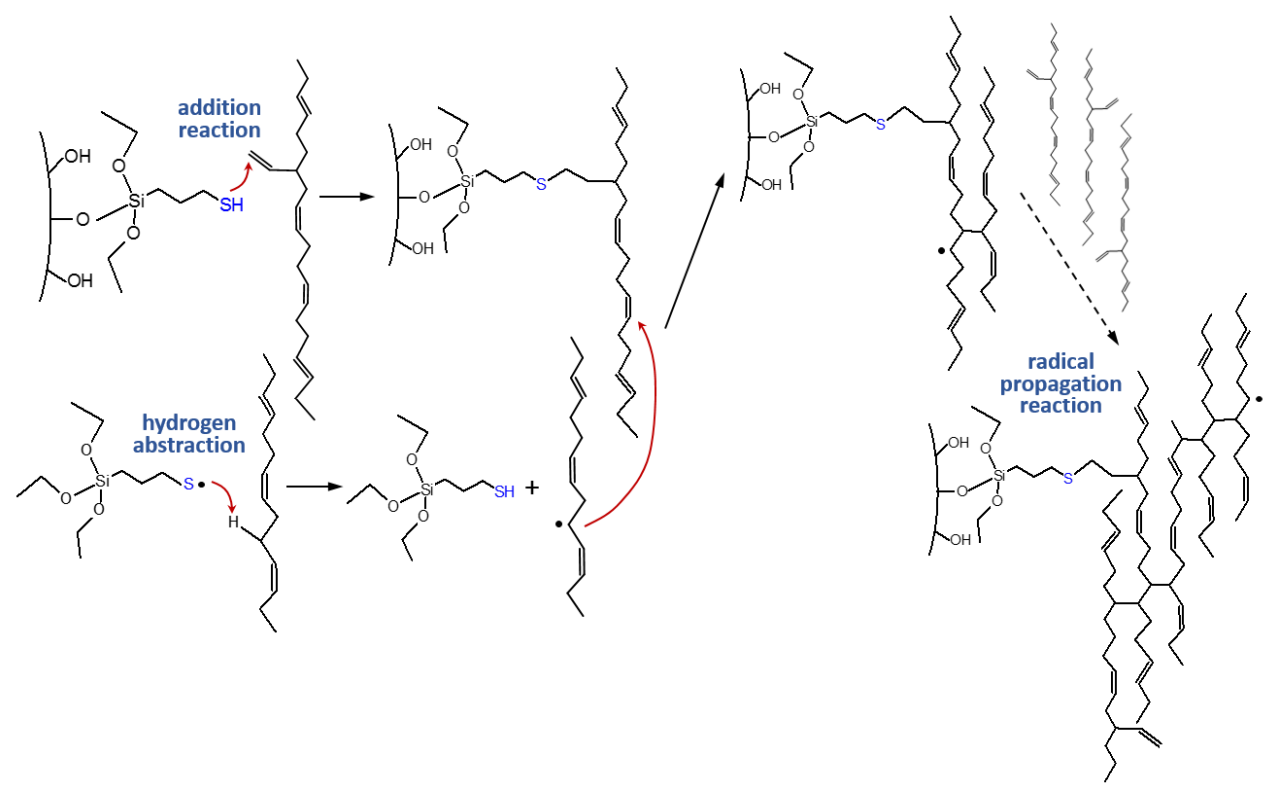

Figure 6-6: Possible mechanism for a quick generation of large amounts of dense chemically bound rubber in the Si 263 system

\section{6-3-3 $\tan \delta$ at $60^{\circ} \mathrm{C}$, Payne effect and Mooney viscosity}

Another important point is, how these differences regarding the generation rate and structure of the chemically bound rubber affect the physical properties of the silica filled compound.

Figure 6-7(a) shows tan $\delta$ values at $60^{\circ} \mathrm{C}$ as a function of the mixing time of the 1 st mixing stage. Only small differences can be observed between Si 263 and Si 69 containing compounds which dumped at short mixing times (less than $150 \mathrm{~s}$ ) which are related to temperatures lower than $135^{\circ} \mathrm{C}$ except the VSL5025-2 / Si 69 system. However, lower tan $\delta$ values are detected for the Si 263 compounds with longer mixing times compared to those of the Si 69 compounds. The shortage of the silanization reaction in both systems can be the reason for that almost no differences are observed in short time mixing compounds. Moreover, the lower average molecular weight of VSL5025-2 compared to others could be suggested as one of the possible reasons for the highest tan $\delta$ value in the VSL5025-2 / Si 69 system. The lower molecular weight means the large number of chain ends in the system, which result in the higher energy loss. However, this also implies that since the VSL5025-2 
/ Si 263 system doesn't show such a high tan $\delta$ value compared to the other Si 263 systems, a different mechanism seems to be working in the system to compensate the influence of the low molecular weight. The specialty in this combination will be described later.

(a)

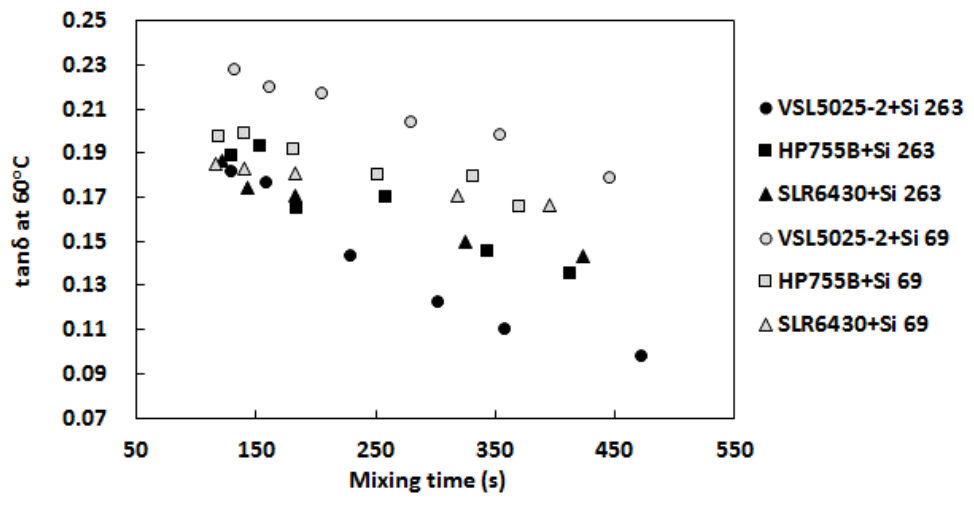

(b)

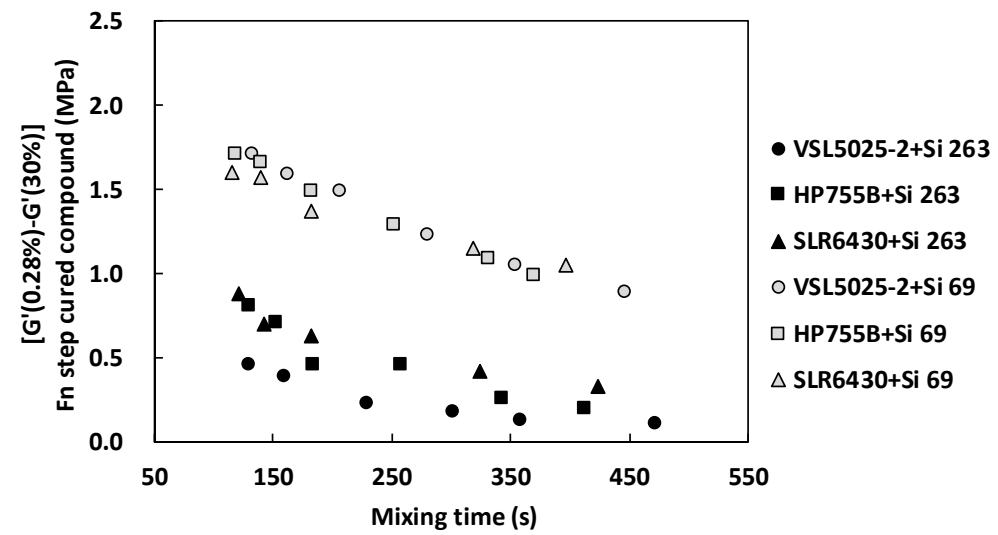

Figure 6-7: (a) Payne effect values of cured compounds and (b) tan $\delta$ values at $60{ }^{\circ} \mathrm{C}$ as a function of the mixing time at the 1st mixing step

The Payne effect values of the cured final compounds, which are an indicator for the fillerfiller interaction [7] are plotted in Figure 6-8 versus the tan $\delta$ values at $60{ }^{\circ} \mathrm{C}$. 


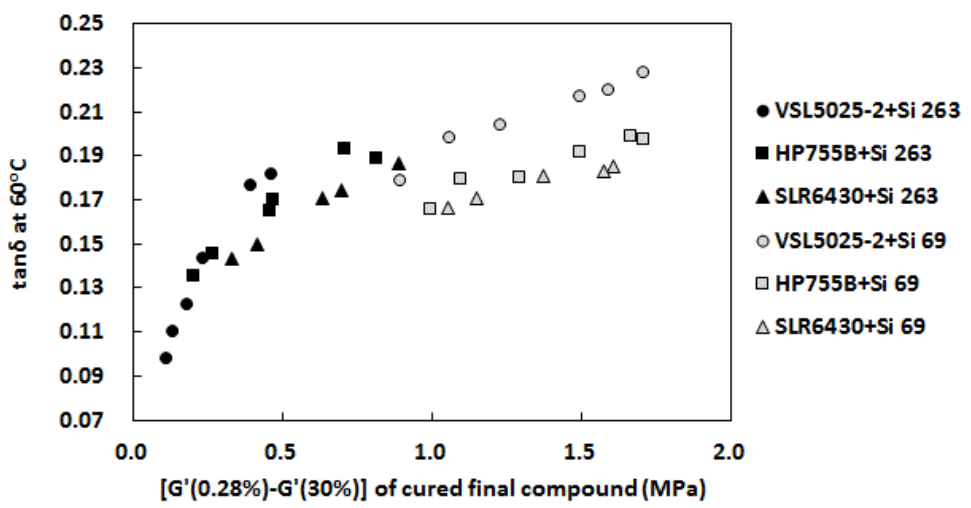

Figure 6-8: Payne effect values of cured compounds vs. tan $\delta$ at $60^{\circ} \mathrm{C}$

The expected tendency that the compound which has a lower Payne effect value has also a lower $\tan \delta$ value at $60^{\circ} \mathrm{C}$ can be observed. Since there are several factors which determine the tan $\delta$ value, though it cannot be explained completely only by the Payne effect values, it is confirmed that at least the filler-filler interaction is one of the main influences which causes an energy loss in the compound. The lower Payne effect values are observed in $\mathrm{Si}$ 263 compounds, consequently, as well as lower tan $\delta$ values at $60^{\circ} \mathrm{C}$.

However, this advantage for lower Payne effect values in Si 263 compounds are not observed when the Payne effect values for the 1st step uncured compounds are plotted instead of those for the cured final compounds, as shown in Figure 6-9.

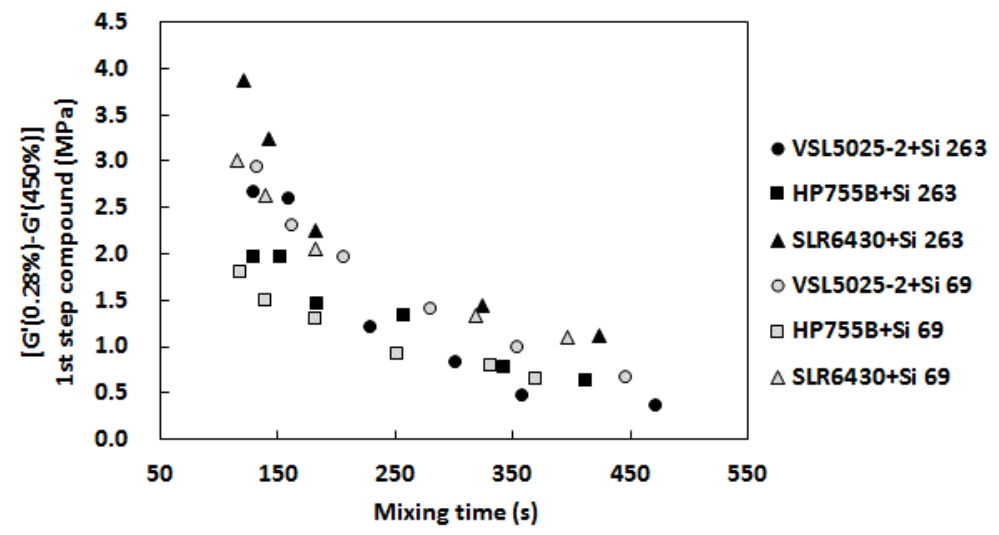

Figure 6-9: Payne effect values for the 1st step uncured compounds as a function of the mixing time at the 1 st mixing step 
This difference can be explained by observing the silica flocculation behavior during heating. Figure 6-10 gives one example for the flocculation test at $130{ }^{\circ} \mathrm{C}$. G' at $1 \%$ shear strain is measured at frequency $60 \mathrm{cpm}$ for $10 \mathrm{~min}$, and the value continuously increases during the test. The silica flocculation is known as a physical phenomenon, [8,2] thus the reason of increasing $G^{\prime}$ during the test is because the mobility of silica clusters to try to reagglomerate is boosted by heating. However, the increasing behavior of the $\mathrm{G}^{\prime}$ value is not constant for all samples. As shown in Figure 6-11, the $\Delta \mathrm{G}^{\prime}$ value between the 10 and 0.2 min in the test decreases with increasing mixing time at the 1st mixing step. Moreover, totally the lower $\Delta \mathrm{G}^{\prime}$ values are observed in Si 263 compounds compared to Si 69 compounds.

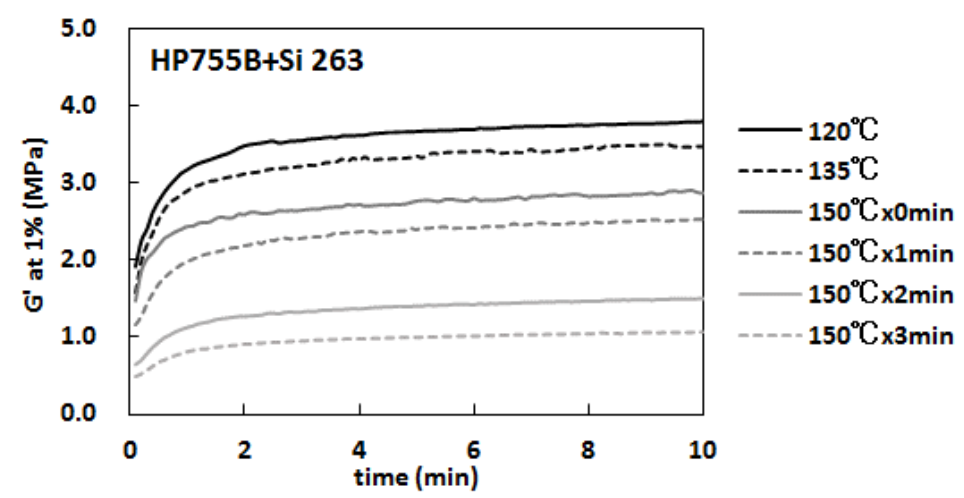

Figure 6-10: Example of $\mathrm{G}^{\prime}$ evolution during a flocculation test at $130{ }^{\circ} \mathrm{C}$

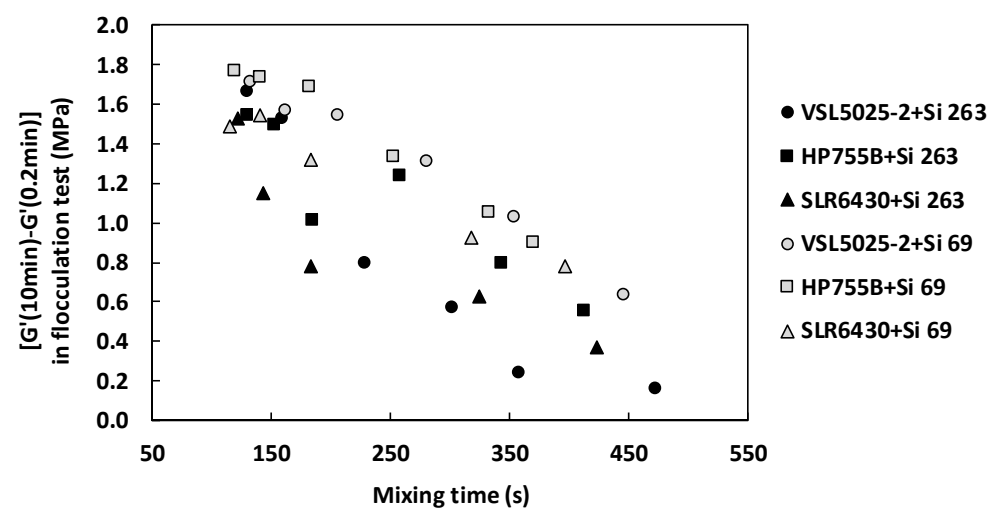

Figure 6-11: The relationship between $\Delta \mathrm{G}^{\prime}$ (at $10 \mathrm{~min}-0.2 \mathrm{~min}$ ) in flocculation test and mixing time at the 1st mixing step. 
When the Payne effect of the 1st step compounds is measured again after heating at 170 ${ }^{\circ} \mathrm{C}$ for $10 \mathrm{~min}$, the same tendency can be obtained with a clearer difference between silanes because of the higher temperature. In this case, the advantage of the Payne effect values in Si 263 compounds, which was not obtained in the compounds before heating, can be observed, as shown in Figure 6-12.

In order to hinder the silica flocculation, which is a physical phenomenon, reducing the attractive force between silica clusters and changing the silica cluster's surrounding to the state which is difficult for the silica to move would be the effective ways.

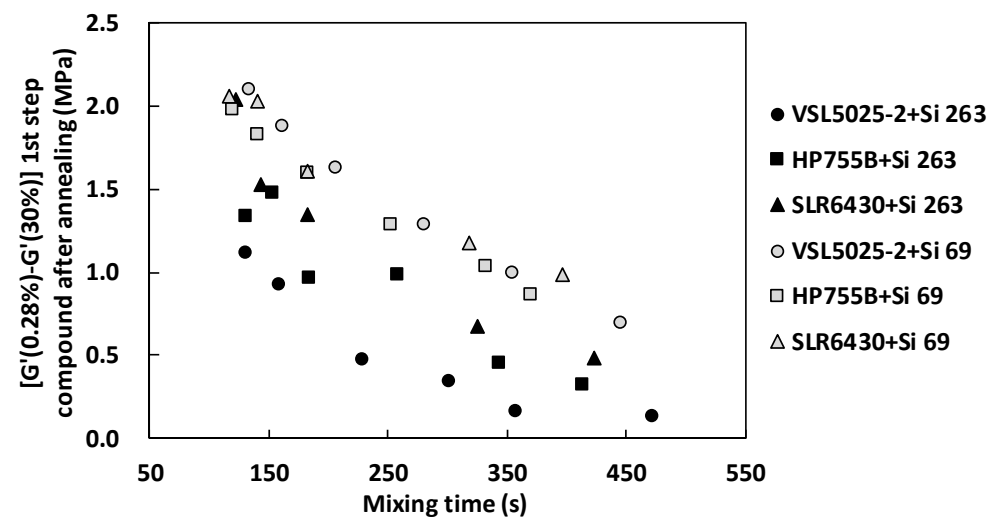

Figure 6-12: The Payne effect of 1st mixing step compounds after annealing

As known well, increasing silanization reaction surely works to reduce the attractive force between silica clusters, which is called hydrophobation effect. At the same time, with increasing the bound rubber content, the possibility to cover the silica surface by the polymer molecule also increases, which also contributes to reduce the attractive force between silica clusters (shielding effect) (Figure 6-13). Furthermore, if the bound rubber network between silica clusters is dense and developed enough, the silica clusters are restricted to approach each other. These effects should be more enhanced in Si 263 systems, which result in a higher suppression effect of silica flocculation during heating (see Figure 6-14). As a consequence, the silica clusters can maintain in a better dispersed state. 


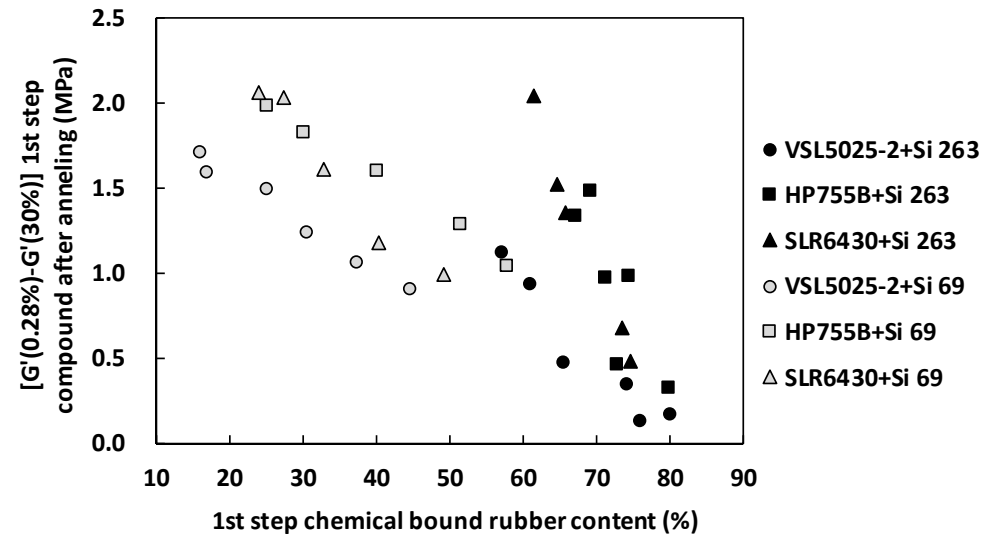

Figure 6-13: The relationship between the Payne effect of 1st mixing step compounds after annealing and 1st step chemical bound rubber content

Si 69 system bound rubber structure

Low amount and loose structure

Si 263 system bound rubber structure

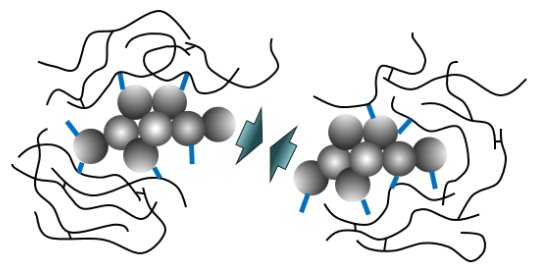

High amount:

1. Large silica surface area covered by polymer

$\Rightarrow$ Reduction of attractive force between silica clusters

2. Formation of bound rubber network

$\Rightarrow$ Restriction of movement potential of silica clusters

$\downarrow$

Dense network and more filler / polymer couplings enhance these effects.

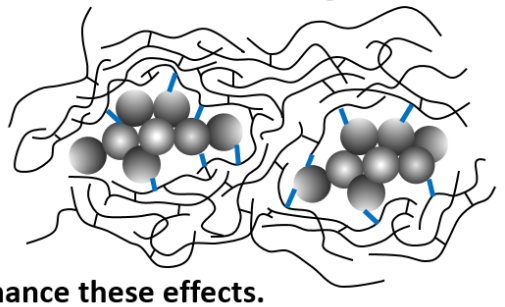

Figure 6-14: Depicted possible explanation for different flocculation behavior between $\mathrm{Si}$ 263 and Si 69 systems

The short mixing times compounds in the Si 263 system gives a lower flocculation suppression ability compared to the longer mixing times compounds in the Si 69 system, even though the Si 263 compounds have already 60 to $70 \%$ of chemical bound rubber content (Figure 6-13). This shows the importance of the silanization reaction. In the mechanism for the SBR / Si 263 system which has been explained so far, it can be assumed that even if the amount of silane which has reacted with the silica surface is not so much, the bound rubber (more exactly, the insoluble part in toluene) can be increased till quite 
high degree in the system because of the radical chain reaction between polymers. However, the high amount of insoluble part of polymer doesn't necessarily guarantee the enough suppression of the silica flocculation. The reaction of the polymer with the silane which coupled to the silica surface is the key point to give the effective shielding effect by the polymer to the silica surface. Therefore, it can be concluded that the important point is that the denser bound rubber is formed on the wide range of the silica surface based on the enough amount of the silanization reaction.

Another discussion point is that the Si 263 / VSL5025-2 system shows the lowest degree of the flocculation, although the chemically bound rubber content in the system was not especially higher compared to other SBR systems (Figure 6-1). This could be explained like the following: Since the number of the points where rubber couples to the silica surface via silane can become higher in the presence of a higher amount of vinyl groups, these chemical couplings increase the above described shielding effect of the silica surface and also the restriction of the silica movement potential. Also, the fact that this polymer has a less bulky structure because of lower content of styrene groups may contribute to heighten the reaction possibility between the polymer and the silane on the silica surface due to a higher accessibility of the vinyl groups. As mentioned before, the VSL5025-2 / Si 69 system showed lower chemical bound rubber content compared to other SBR / Si 69 systems, however the value in the VSL5025-2 / Si 263 system was similar value to other SBR / Si 263 systems. This fact seems to support indirectly the effect of the direct reaction between a mercapto group and a vinyl group. By this reaction which doesn't occur in the Si 69 system, even the VSL5025-2 system which has lowest molecular weight can create similar level of a chemical bound rubber content.

Moreover, the VSL5025-2 / Si 263 system shows also slightly lower the Payne effect value of the 1st step uncured compound in the longer mixing time compared to other systems. Since this is the result of the measurement before vulcanization, this difference should correspond to the silica dispersion level obtained in the mixing step. This may be because the polymer coupled with a silane acted as a main chain modified polymer leading to the acceleration of the silica dispersion. $[9,10]$. In other words, if the polymer couples with the silica cluster, the polymer for which the mechanical shear force are applied during mixing can deform with taking the silica clusters, which helps to break the silica cluster to the smaller size. 
These could be considered as the main reason for getting a better silica dispersion state in the SBR / mercapto-silane compounds. However, the developed bound rubber network can also cause a lower flexibility (or plasticity) of the uncured compound, which leads to the observed worse processability. In fact, the Mooney viscosity of the 1st mixing stage shows the following behavior: After reaching a mixing temperature of $150{ }^{\circ} \mathrm{C}$, the Mooney viscosities in the Si 263 compounds basically increase with increasing mixing time but those in the Si 69 compounds decreases with increasing mixing time (Figure 6-15). These differences can be explained by the above described the bound rubber developing mechanism in the Si 263 system. Si 263 can increase the coupling points between silica and rubber by the direct addition reaction to the double bond and also accelerate the SBR radical chain reaction by working as a subtracter of an allylic hydrogen from the polymer, which results in the formation of the denser polymer network in the system. With increasing mixing time at a high temperature, this tendency should be enhanced. Although the Mooney viscosity only in the VSL5025-2 / Si 263 system decreases after reaching a mixing time of $250 \mathrm{~s}$, the following reasons for this behavior can be considered. The dump appearance of the longer mixing time compounds in the system shows already a powderlike condition, as shown in Figure 6-16. Therefore, the Mooney viscosity may have not been able to be measured properly anymore. This powder-like condition may be caused by the combination of large amounts of chemical linkages between the polymer and silica via silane, the dense bound rubber network and also the lowest molecular weight of the polymer. The lower molecular weight of the polymer should have a lower entanglement ability to other polymer molecules due to the shorter length of the molecular chain. Therefore, when it is attached at the silica surface, it behaves more like a filler by losing the viscous behavior. Taking all three possible explanations into consideration, the observed increasing Mooney viscosity behavior at longer mixing time supports the presented idea of the development of a denser bound rubber network in the Si 263 system. 


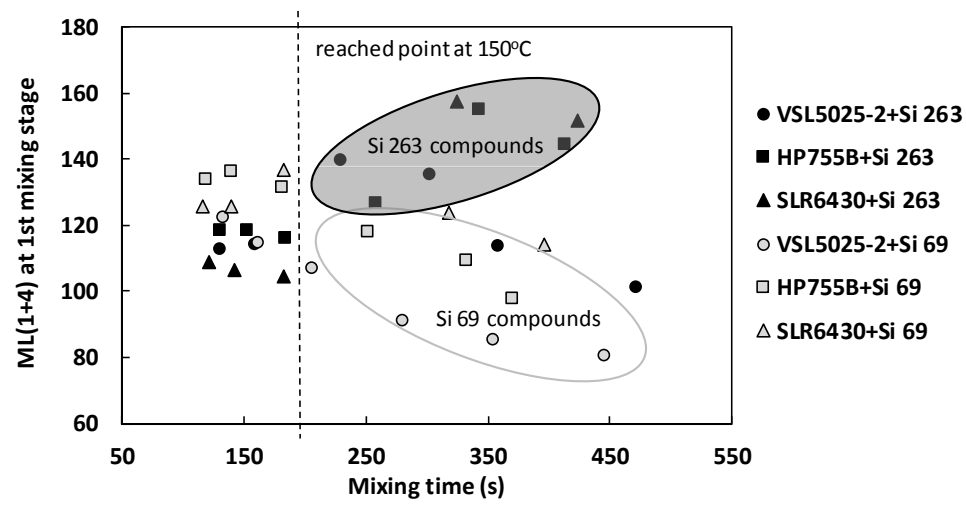

Figure 6-15: Mooney Viscosity of 1st mixing step compounds as a function of mixing time

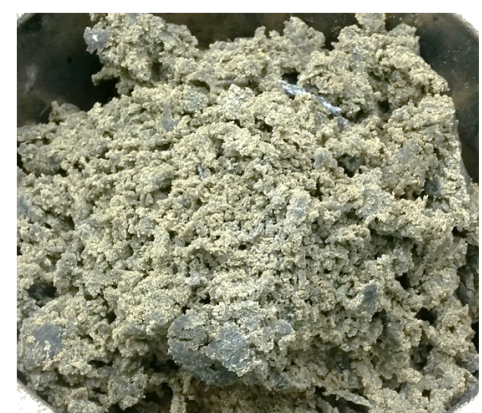

Figure 6-16: Dump appearance of the VSL5025-2 / Si 263 compound with the longest mixing time at the $1^{\text {st }}$ mixing stage

\section{6-4 Conclusions}

The evaluation of the SBR-BR / Si 263 or Si 69 compounds confirmed that the Si 263 compounds create larger amounts of bound rubber even with shorter mixing time compared to the Si 69 compounds, where most of the bound rubber consists of chemically bound rubber type. In addition, the swelling measurement reveals that the chemically bound rubber in the Si 263 compound has formed a significantly denser polymer network. Considering the mechanism based on the findings in the model study, it seems to be because of the very high addition reactivity of the mercapto-silane radical to the rubber polymer double bond, especially to vinyl and cis double bonds. This is combined with the 
high radical chain reactivity of the polymer radicals generated by the reversible addition reaction and the abstraction of the allylic hydrogen by the mercapto-silane radicals. The continuation of the radical chain reaction leads to the creation of large amount of the $\mathrm{C}-\mathrm{C}$ bonds resulting in a dense polymer network.

The Si 263 compounds provided at the same mixing time lower tan $\delta$ at $60{ }^{\circ} \mathrm{C}$ compared to the Si 69 compounds. Additionally, the values seem to correlate with the Payne effect values of the cured compounds. This indicates that the filler-filler interaction is one of the dominant factors to determine the tan $\delta$ at $60{ }^{\circ} \mathrm{C}$. However, almost no difference was observed in the Payne effect values of the uncured compounds between the Si 263 and the Si 69 compounds. By the experiments to monitor the flocculation behavior of the uncured compounds, it was confirmed that the Si 263 compounds flocculate less, which result in lower Payne effect values as well as lower tan $\delta$ values at $60^{\circ} \mathrm{C}$ after the vulcanization. This difference seems to be because of the large amount of chemically bound rubber content together with its dense and developed structure in the mercapto-silane compounds. The bound rubber shields the silica surface, moreover, the formed dense and developed structure reduces the the reagglomeration tendency of silica clusters. By this mechanism, the flocculation of the silica clusters are suppressed resulting in the maintained better silica dispersion state during the vulcanization. These could be considered as the main reason for getting a better silica dispersion state in SBR / mercapto-silane compounds compared to SBR / sulfide-silane compounds.

The suppression of the silica flocculation was observed most clearly in the high-vinyl type of SBR / Si 263 system. This seems to be because of the largest amount of bonding points between silica and polymer via silane. But this system did not show the highest amount of chemically bound rubber. Considering the dominant factors to determine the amount of the chemically bound rubber, the following three reasons should be taken into account. They are the number of the coupling points between the polymer and the silica via the silane, the molecular weight of the polymer and also the growth of the polymer layer surrounding the silica surface. For the first one, the high-vinyl type of SBR seems to have the highest number of polymer-silane coupling points, as mentioned before. In this experiment, the lower-vinyl type of SBR, having less polymer-silane coupling points, has a higher molecular weight. Furthermore, the swelling measurement of the chemically bound rubber shows a similar polymer network density in all three SBR systems, which means that these three SBRs have a similar level of radical chain reactivity. The combination of the above three 
points seems to lead to a similar amount of the chemically bound rubber in all three SBR / Si 263 systems.

In addition, in all SBR / Si 263 systems, the increase of the Mooney viscosity or the tendency to worsen the dump compound appearance with increasing mixing time was observed. This indicates that the large amounts of chemical linkages between polymer and silica via silane in combination with the developed dense bound rubber networks cause a lower flexibility (or plasticity) of the uncured compounds, which leads to the observed worse processability.

Taken as a whole, increasing the direct bonding between silica and polymer via silane in order to obtain a sufficient shielding effect by polymer at the silica surface together with preventing the formation of a too dense bound rubber network caused by the excess amount of $\mathrm{C}-\mathrm{C}$ bonding seem to be the better direction to balance the silica dispersion and the processability. 


\section{6-5 References}

[1] S. Wolff, M.J. Wang, E.H. Tan, Rubber Chem. Technol., 66 (1993) 163-177

[2] S. Mihara, "Reactive processing of silica-reinforced tire rubber: new insight into the time and temperature-dependence of silica rubber interaction", PhD. Thesis: 2009, Dept. of Elastomer Technology and Engineering, Univ. of Twente, Enschede, the Netherlands.

[3] F. Schapman, J. P. Couvercelle, C. Bunel, Polymer, 39 (1998) 4955-4962

[4] B. M. E. van der Hoff, Rubber Chem. Technol., 38 (1965) 560-572

[5] Y. Minoura, Journal of the Society of Rubber Science and Technology, Japan, 40 (1967) 820-855

[6] J. Kruzelak, R. Sykora, I. Hdec, Rubber Chem. Technol., 90 (2017) 60-88

[7] A. R. Payne, Rubber Plast. Age, 42 (1961) 963

[8] C.J. Lin, W.L. Hergenrother, E. Alexanian, G.G.A. Böhm, Rubber Chem. Technol., 75 (2002) 865-890

[9] S. Maghami, W.K. Dierkes, J.W.M. Noodermeer, Rubber Chem. Technol., 89 (2016) 559572

[10] M. Hayashi, H. Hama, K. Inagaki, sumitomo chemical technical report, Accessed day: 07/03/2018, https://www.sumitomo-chem.co.jp/rd/report/theses/docs/2011-1J_3.pdf 


\section{Chapter 7}

\section{Rubber compound study-2; \\ Comparison of silica filled NR or IR compounds with silica filled SBR/BR compound in both silane systems}

\section{7-1 Introduction}

Since the silica / silane technology was introduced in the tire industry, the usage and the further improvement of the technology have been targeted mainly on the passenger car (PC) tire tread compounds where SBR and BR is used as main polymers. However, with an increasing demand of tire performances, the development to apply this technology also for the truck and bus (TB) tire tread compounds which use NR as the main polymer has recently received increased attention. NR is essential for the TB tire tread compound because of the advantage of the superior mechanical properties such as tensile strength and tear strength resulting from the strain induced crystallization coming from the high regularity of the micro structure together with the substantial amount of the high molecular weight components. The high abrasion resistance is one of the most important requirements for the TB tires. Hence, a high surface area of carbon black (CB) which can expect a high reinforcing effect is generally used in order to fulfill this requirement. This principle should be the same in the case of silica, therefore, a high surface area silica which is normally more difficult to be dispersed in the non-polar rubber should be used. Recently, a very high surface of silica which has the CTAB value of $250 \mathrm{~m}^{2} / \mathrm{g}$ has become commercially available in the market and the effectiveness for improving both rolling resistance and abrasion resistance has been reported.[1]

It was observed that the improvement of properties by replacing the CB by the silica / silane system is normally lower inside the NR compounds than that for the SBR / BR compounds. The competition for the adsorption on the silica surface between silanes and proteins which are impurities in NR has been considered to lead to a weaker silica / silane / polymer coupling.[2] However, as dicussed in the previous chapter 6, by considering the fact that the type of the silane affects the chemically bound rubber content and the 
subsequent silica re-agglomeration behavior, it can be assumed that the different molecular structure between an isoprene and a butadiene type of rubber can also be one of the factors to determine the effectiveness of the silica / silane system.

As mentioned before, the technology development to improve the achievable dispersion level of the high surface area silica in the NR system by improving the coupling efficiency is significantly important to fulfill the increased requirement level. For this purpose, it is crucial to understand the reinforcing mechanism of silica in NR for both sulfide- and mercapto-silane systems. Therefore, an evaluation of the silica filled NR or IR compounds in comparison with the silica filled SBR / BR compounds was carried out in order to clarify if the obtained knowledge in the previous chapters regarding the SBR / BR systems can also be applied for the NR or IR system.

\section{7-2 Experimental}

\section{7-2-1 Material and Compound Formulations}

By combining NR or IR with Si 69 or Si 263, four different formulation compounds were prepared. The natural rubber used was Ribbed Smoked Sheet 3 (RSS3) and the isoprene rubber used was Nipol IR2200 (cis content > $98 \%$ ). The compound formulation is shown in Table 7-1, which is the same as used for the SBR study in Chapter 6 except the applied polymer.

Table 7-1: Compound formulation

\begin{tabular}{|l|c|l|}
\hline 1st stage & (phr) & \\
\hline NR or IR & 100 & Polymer \\
\hline ULTRASIL 7000 GR & 80 & HD silica \\
\hline Stearic Acid & 2 & Activator \\
\hline Nocrac 6C & 2 & Anti aging agent \\
\hline Si 69 or Si 263 & 6.4 & Silane coupling agent \\
\hline TDAE Oil & 15 & Oil \\
\hline 2nd stage & & \\
\hline ZnO & 2.5 & Activator \\
\hline 3rd stage & & \\
\hline Sulfur & 1.5 & \\
CBS & 1.7 & Accelerator \\
DPG & 2 or 0 & Secondary Accelerator \\
\hline
\end{tabular}




\section{7-2-2 Mixing}

The applied mixing conditions were also the same as those used in the SBR study (Chapter 6).

Table 7-2: Mixing protocol

\begin{tabular}{|c|c|c|}
\hline \multicolumn{3}{|c|}{ 1st stage (Intermesh mixer $(1.5 \mathrm{~L})$, revolution varied) } \\
\hline & Total mixing time & Action \\
\hline 1 & Os & polymer \\
\hline 2 & $20 \mathrm{~s}$ & silica, St-Ac, antioxidant, silane, Oil \\
\hline 3 & $70 \mathrm{~s}$ & ram up, sweep, ram down \\
\hline 4 & \multicolumn{2}{|c|}{$\begin{array}{l}\text { 1) Experiments were carried out with varying mixing time } \\
\text { 2) Rotor revolution was adjusted in order to keep about } 150^{\circ} \mathrm{C}\end{array}$} \\
\hline \multicolumn{3}{|c|}{ 2nd stage (Intermesh mixer (1.5L), 30rpm (fixed)) } \\
\hline & Total mixing time & Action \\
\hline 1 & Os & batch 1st stage, $\mathrm{ZnO}$ \\
\hline 2 & $25 s$ & ram up, sweep, ram down \\
\hline 3 & $70 \mathrm{~s}$ & ram up, sweep, ram down \\
\hline 4 & $115 s$ & Dump out \\
\hline \multicolumn{3}{|c|}{ 3rd stage } \\
\hline \multicolumn{3}{|c|}{ Add curatives on a two roll mill } \\
\hline
\end{tabular}

\section{7-2-3 Characterization methods}

The Mooney viscosity $\mathrm{ML}(1+4)$, the bound rubber, the swelling degree of chemically bound rubber by toluene, the Payne effect, the flocculation and the viscoelasticity measurements of these rubber compounds were carried out following the same setting as described already in Chapter 6.

Additionally, the swelling degree of cured compounds by toluene was measured as following: The cured sample was immersed in toluene for $72 \mathrm{~h}$ at room temperature. The sample was taken out of toluene and weighed after drying the surface of the sample with filter paper to remove the outer attached toluene. The swelling degree was calculated by the following equations (7-1):

$$
\text { Swelling degree }(\%)=\frac{V-V_{0}}{V_{0}} \times 100 \quad(7-1)
$$


where $V_{0}$ and $V$ are the volumes of the sample before and after immersed in toluene, respectively.

The sulfur content in the cured samples after acetone extraction was measured using the auto sulfur analyzer (EMIA-920V / FA, HORIBA). The samples were put in the crucible and heated under oxygen atmosphere, subsequently the released sulfur dioxide was measured by an IR detection system. The amount of sulfur was calculated relative to the weight of the sample.

\section{7-3 Results and Discussion}

\section{7-3-1 Bound rubber content before and after ammonia treatment}

The overall bound rubber content in the NR or IR systems is shown in Figure 7-1, and for comparison, the results inside the SBR systems are also shown on the right side. As already observed in the SBR systems, the bound rubber content increases with increasing mixing time also in the NR and IR systems. An exception is the NR / Si 263 system. NR systems show a higher bound rubber content than IR systems in both silane formulations, and this tendency is explicitely clear for the short mixing range. This could be associated with the following characteristics of NR which is a natural product. Firstly, it is known that NR already contains a higher gel content compared to IR. $[3,4]$ The proteins and phospholipids, which are contained in NR, behave like network points for the NR chains. It is assumed that these contribute to the gel formation and branching in NR, as shown in Figure 7-2.[3] Secondly, there might be the possibility that protein and / or phospholipids reacts with the silane coupling agent, which also contributes to the increase of the bound rubber content in NR systems.[5]

Si 263 compounds with short mixing time show higher bound rubber contents than Si 69 compounds, however, the values inside both silane systems reach a similar level when the mixing time reaches $250 \mathrm{~s}$, after that, the values stay on the same level. This is one of the different tendencies from the SBR systems, that the bound rubber content in Si 263 systems show higher values than that in Si 69 systems for all mixing times. Another noticeable difference from SBR systems is the maximum bound rubber content. The maximum value inside SBR / Si 263 compounds was about $80 \%$, whereas it is 60 to $70 \%$ inside the NR or IR 
compounds with both silanes, which is a similar level as that inside the SBR / Si 69 compounds expect for the VSL5025-2 / Si 69 compounds. The Si 263 compounds with NR or IR do not reach the same bound rubber content level as the SBR / Si 263 compounds.
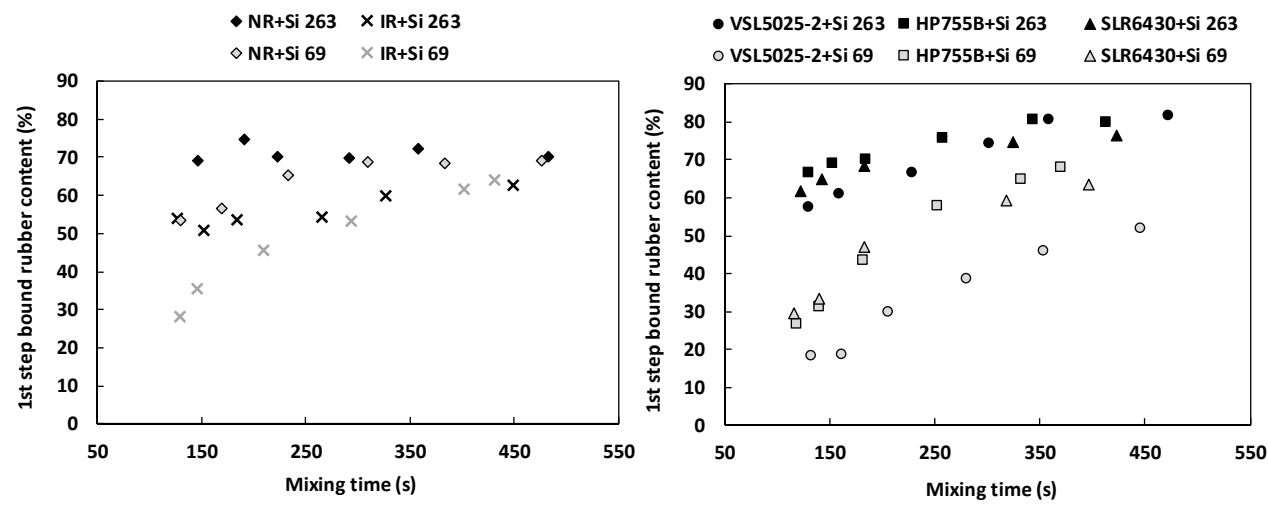

Figure 7-1: Overall bound rubber content of 1st step mixing compounds with different mixing time inside the (a) SBR systems, (b) NR or IR systems

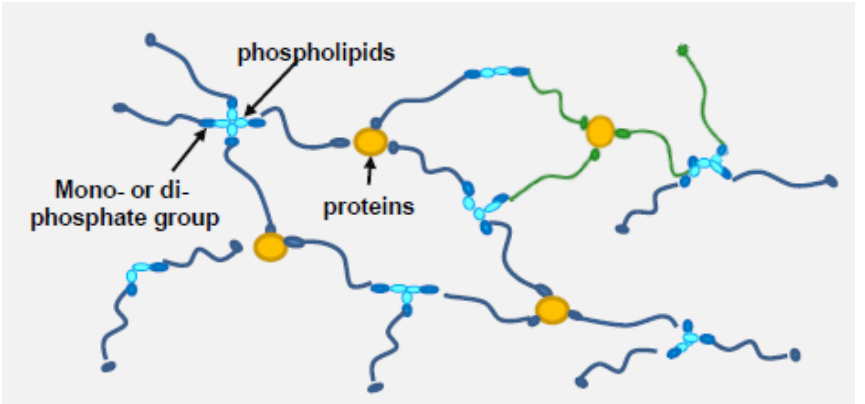

Figure 7-2: Proposed polymer chain structure in NR associated with proteins and phospholipids $[5,6]$

The physically and chemically bound rubber contents which were obtained by the ammonia treatment are shown in Figure 7-3. As a comparison, one of the results in the SBRBR systems is also shown at the bottom of the same figure. 

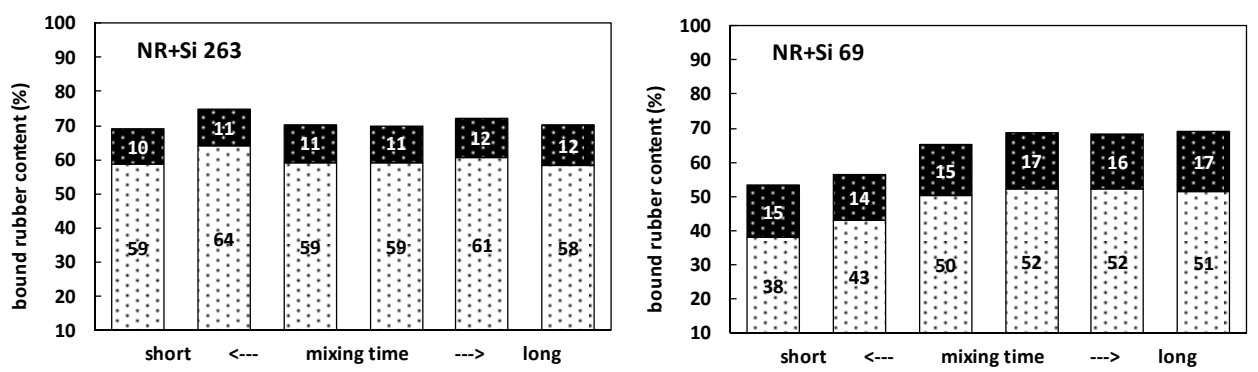

๑Chemically bound rubber (\%) D Physically bound rubber (\%)

$\square$ Chemically bound rubber (\%) Physically bound rubber (\%)
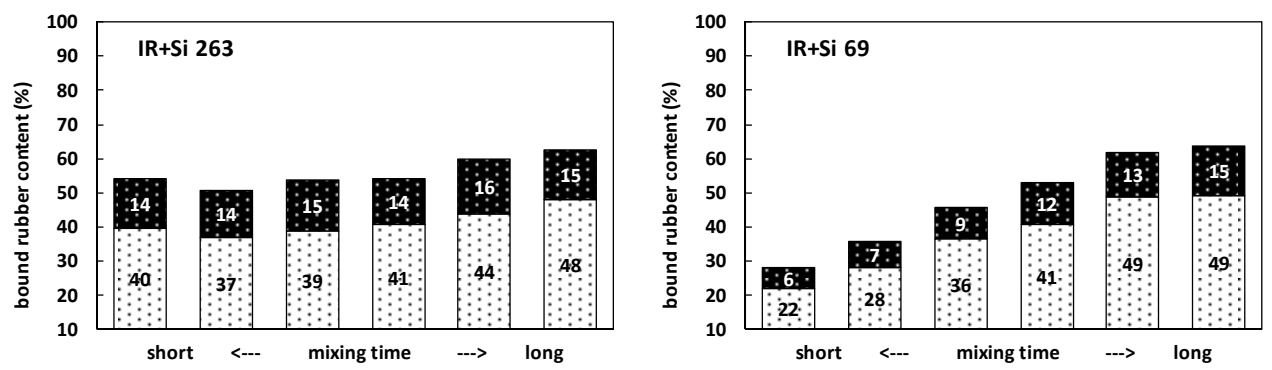

$\square$ Chemically bound rubber (\%) D Physically bound rubber (\%)

$\square$ Chemically bound rubber (\%) • Physically bound rubber (\%)
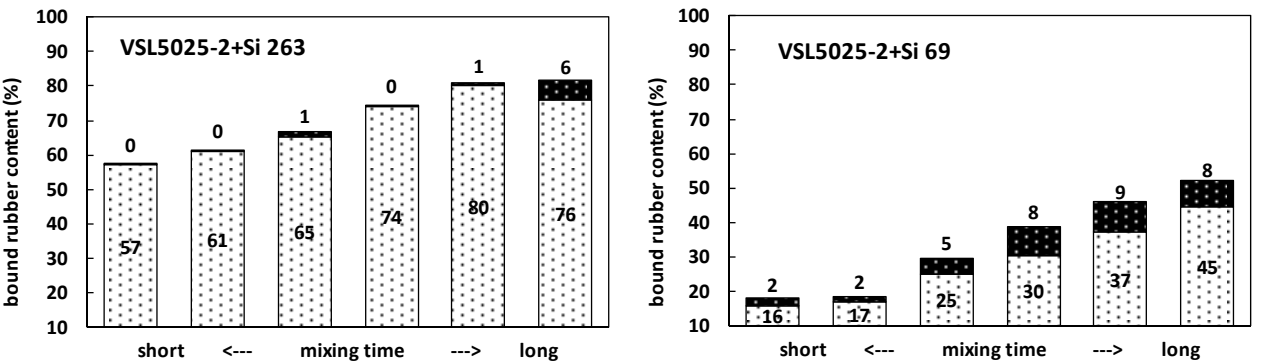

$\square$ Chemically bound rubber (\%) Physically bound rubber (\%)

$\square$ Chemically bound rubber (\%) @ Physically bound rubber (\%)

Figure 7-3: Physically and chemically bound rubber content of 1st step mixing compounds

Although only considerably small amounts of physically bound rubber was detected in the SBR / Si 263 systems, more than $10 \%$ of the value was observed in all of the NR or IR / Si 263 systems. Even when the values in the NR or IR / silane systems are comparable, only the values in the IR / Si 69 system with short mixing time are slightly lower. However, the higher overall bound rubber content inside the short mixing time Si 263 compounds compared to that inside the Si 69 compounds is because of the higher generation of chemically bound rubber, which is the same tendency as inside the SBR systems. 
The degree of swelling of chemically bound rubber including the results in the SBR systems is summarized in Figure 7-4. And, assuming that the chemically bound rubber in each compound has a homogeneous structure, the normalized degree of swelling by the chemically bound rubber content is shown in Figure 7-5.

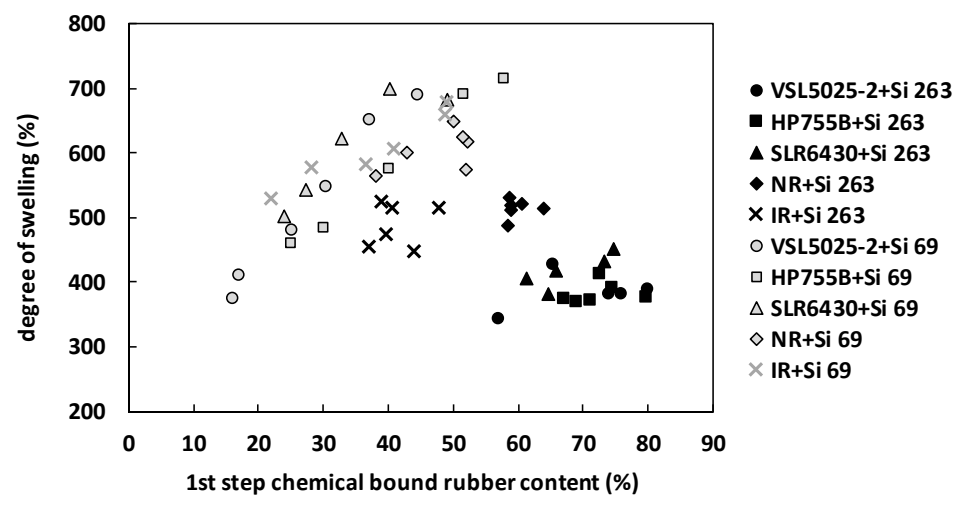

Figure 7-4: Degree of swelling of chemically bound rubber by toluene

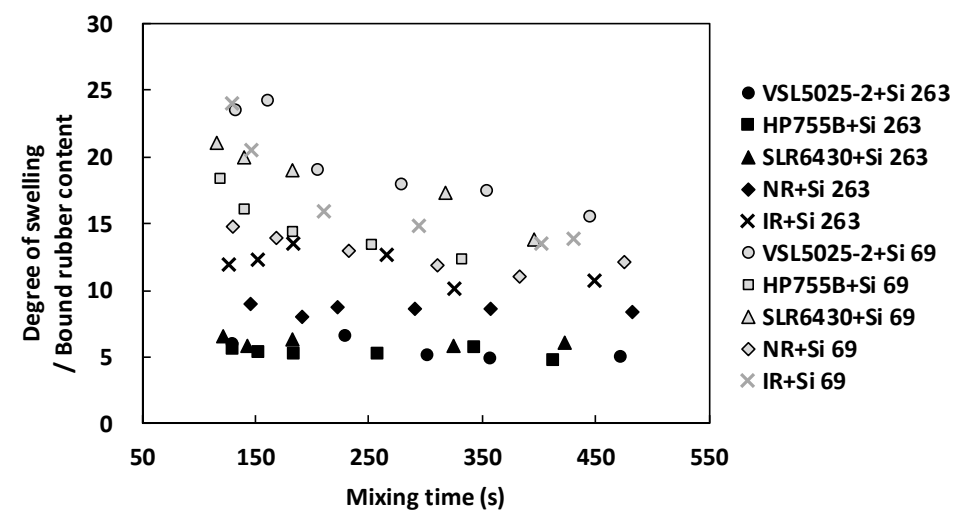

Figure 7-5: Degree of swelling divided by the chemically bound rubber content vs. mixing time

The values of NR or IR / Si 69 compounds are in the same area as those of the SBR /Si 69 compounds, while those of the NR or IR / Si 263 compounds are in the middle between all Si 69 compounds and SBR / Si 263 compounds. There is a certain measurement error, but nevertheless, it is obvious to identify the above mentioned different categories. These results indicate that the chemically bound rubber in the NR or IR / Si 263 compounds leads still to a denser network compared to the Si 69 compounds, however, it is not as dense as that in the SBR / Si 263 system. 
The reason for this difference can be explain as following: In the case of the isoprene type of rubber (NR and IR) systems, there exists three different positions of allylic hydrogen in the structure because of the additional methyl group attached to the double bond. The most labile allylic hydrogen is the number (3) hydrogen in Figure 7-6 (a), because the created radical can be transformed to a tertiary radical by the delocalization effect (Figure 7-6 (b)), which can have a relatively long life duration. [7,8] It is known that because of this longer life duration the coupling reaction between the polymer radicals is preferred in these types of polymers compared to the radical chain reaction, which results in the completion of the radical reaction.[9] Radical processes were intensive studied in peroxide crosslinking systems. Therefore, a comparison of this existing knowledge with the obtained results in this study, can lead to a deeper understanding for the mercapto-silane system. It has been reported that the cure efficiency in the peroxide vulcanization of NR using dicumyl peroxide (DCP) was one, which means that one mole of cross-links was formed by one mole of peroxide (Table 7-3).[9,10] Certain amount of polymer radical chain reaction, which is known to be preferred in the SBR and BR systems, would be able to proceed simultaneously also in the isoprene type of rubber system. However, the coupling reaction has priority in this system. This seems to be a reasonable reason for the lower amount of chemically bound rubber content in the isoprene type of rubber systems compared to the SBR systems (See Figure 7-7).

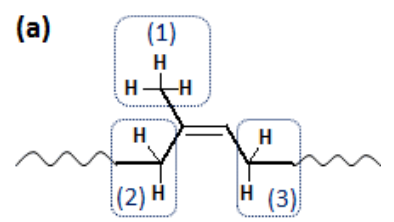

(b)

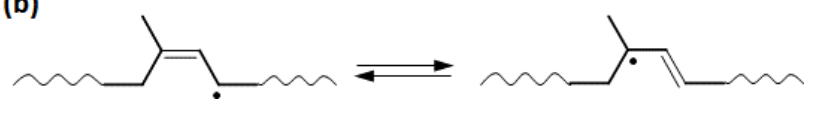

Figure 7-6 (a) allylic hydrogens in isoprene type of rubber, (b) radical resonance stabilization

Table 7-3: Relative cross-linking efficiencies of some elastomers cured with DCP $[9,11]$

\begin{tabular}{lc}
\hline Elastomer & Cross-linking efficiency \\
\hline BR & $\geq 10$ \\
SBR & $\geq 10$ \\
NBR & $\geq 1$ \\
NR & 1 \\
EPM & $0.1-0.8$ \\
CR & 0.5 \\
IIR & 0 \\
\hline
\end{tabular}



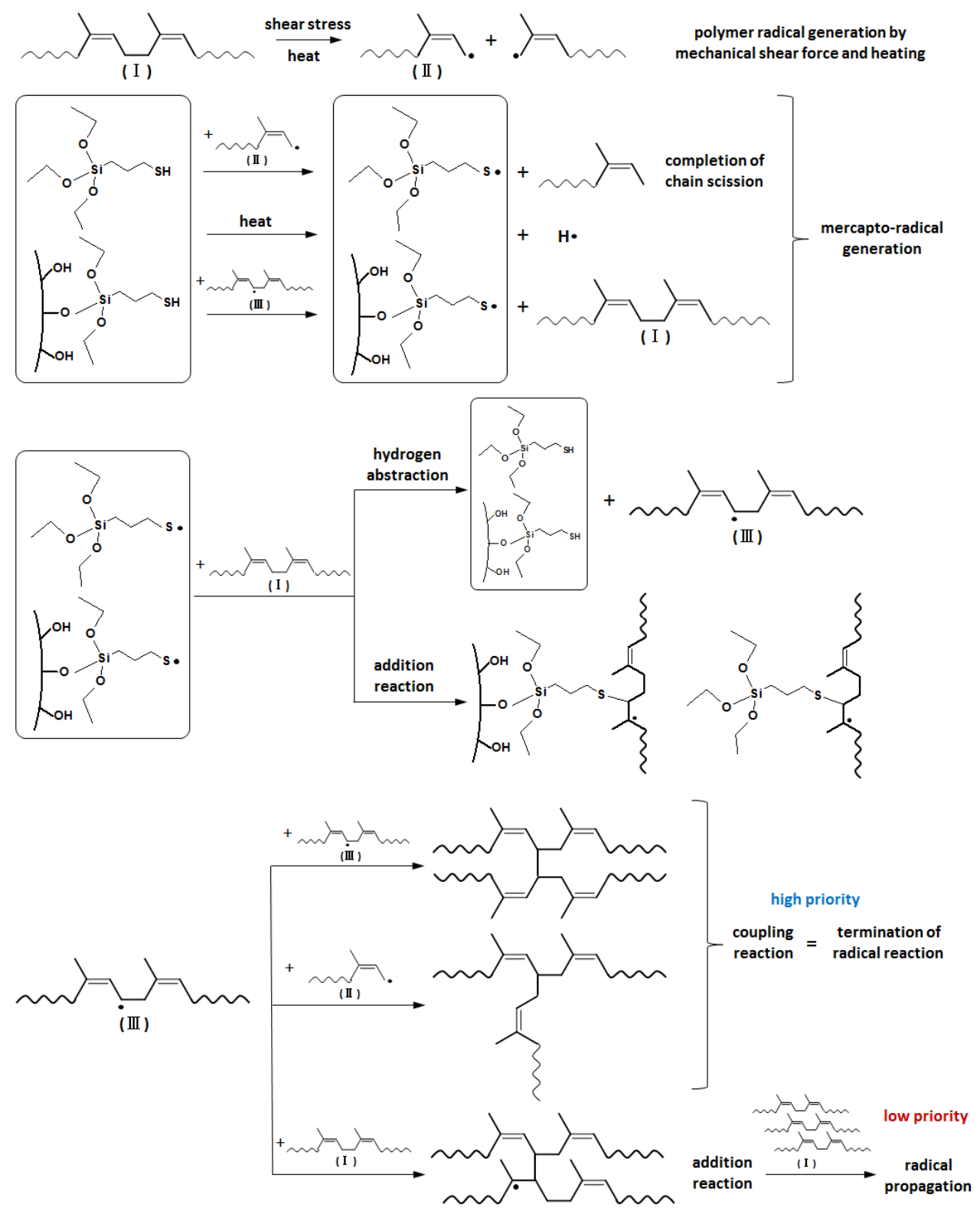

Figure 7-7: Possible reaction mechanism in the isoprene type of rubber and Si 263 system 
A higher amount of carbon radicals inside the polymer can still be produced in the IR / Si 263 system compared to the Si 69 system, because of the higher ability of the mercapto group to abstract an allylic hydrogen and also the addition reaction to the double bond. This should lead to a higher degree of the coupling reaction as well as the radical chain reaction between polymers resulting in an increase of the molecular weight. This can be one of the explanation for the higher chemically bound rubber content in the Si 263 systems with shorter mixing time.

In the model study described in Chapter 3, the addition reaction product of Si 263 to the double bond in the T3M3H was observed only in the GC/MS measurement and not in the NMR spectra, which means that the formation of this reaction product has occurred only to a very low amount. Therefore, it can be assumed that the possibility of the addition reaction of a mercapto-radical to the cis or trans double bond of the isoprene type of rubber can be lower compared to that in vinyl or cis structures in butadiene type of rubber. The degree of easiness for a hydrogen abstraction depends on the stability of the formed radical. Therefore, mercapto-silane radical prefers to abstract allylic hydrogen compared to the addition reaction in this system because of the higher stability of the tertiary radical. Steric hindrance by methyl group to the double bond can also be the reason for the difficulty of the addition reaction. However, it is demonstrated that in the sulfur-sulfenamide vulcanization system a benzothiazol radical (represented as $\mathrm{Bt}-\mathrm{S} \bullet$ ) which is produced by the thermal scission of the sulfonamide accelerator can react with the double bond of the isoprene units as shown in Figure 7-8.[12] Therefore, considering the fact that the rubber system contains a higher ratio of double bonds relative to the amount of mercapto groups compared to the model system, it seems reasonable to suppose that the addition reaction of a mercapto silane radical to the isoprene double bond unit can occur. This also could support the quick generation of a high chemically bound rubber content only in the Si 263 systems.

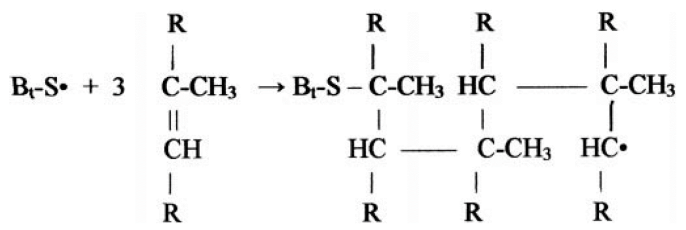

Figure 7-8: Addition reaction of benzothiazol radical with isoprene type of rubber followed by radical chain reaction 
The chemically bound rubber content in both silane systems reaches almost the same level when the dump temperature reaches $150{ }^{\circ} \mathrm{C}$. This may be because of an increasing possibility of a main chain scission reaction with or without oxygen at higher temperature. For example, although the crosslinking and scission are competing in the peroxide vulcanization, it is reported that the scission predominates at higher temperature.[13] The scission of the elastomer backbone is usually promoted by tertiary radicals. $[9,14]$ Therefore, the influence of the main scission reaction in the isoprene type of rubber system should be higher compared to the SBR systems.

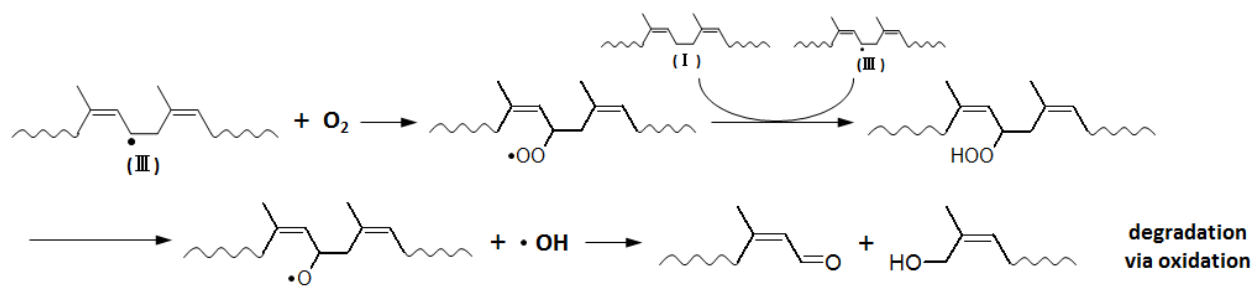

Figure 7-9: Oxidation mechanism of isoprene type of rubber

\section{7-3-2 Payne effect and Mooney viscosity}

Figure 7-10 shows the Payne effect values in the 1st mixing of the uncured compounds. In this case, as already the same tendency was observed in the SBR systems, there is almost no difference of the values between two silanes, and there is even the case that the $\mathrm{Si} 69$ compounds gives a lower value than in the NR systems.

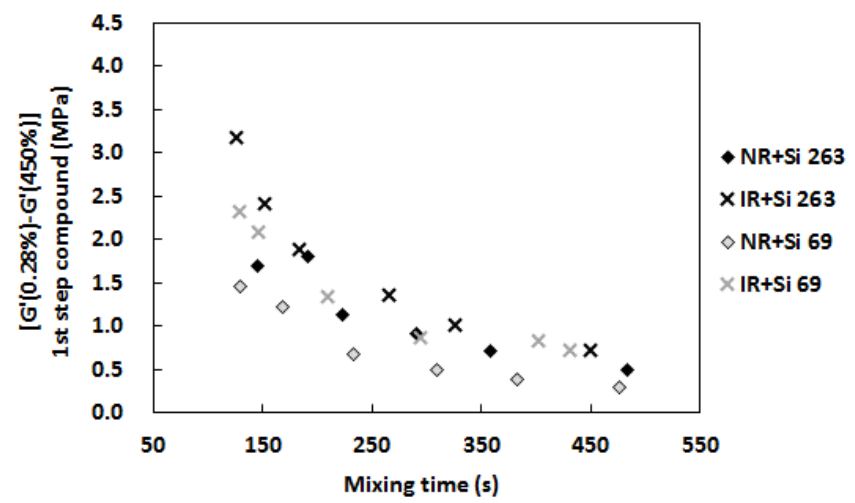

Figure 7-10: Payne effect values for the 1st step uncured compounds as a function of the mixing time at the 1st mixing step 
However, when these compounds are measured again after heating at $170^{\circ} \mathrm{C}$ for $10 \mathrm{~min}$, the Si 263 system shows the lowest Payne effect values in both polymer systems (Figure 711). Since the chemically bound rubber content inside both silane systems is almost on the same value at the longer mixing times, the slightly denser bound rubber structure (See Figure 7-4 and 7-5) and the supposed addition reaction effect between the mercapto-silane and the rubber seems to be responsible for the suppression of the silica re-agglomeration behavior.

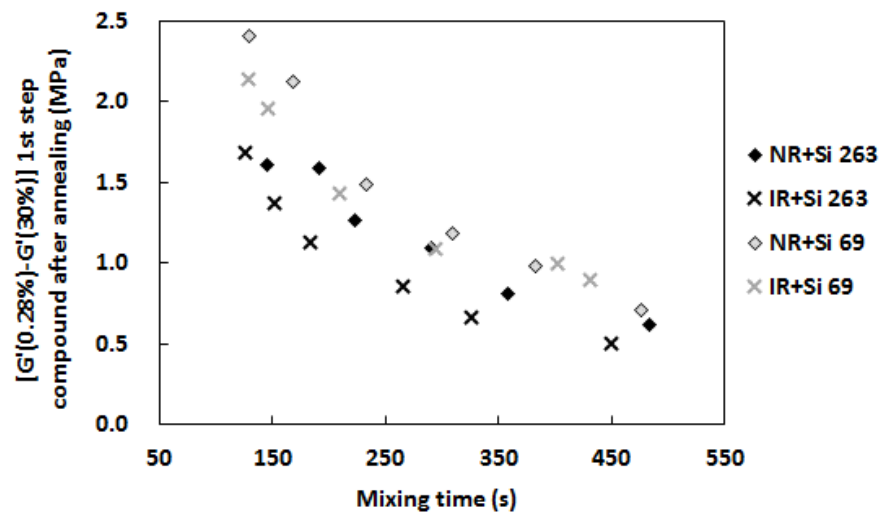

Figure 7-11: The Payne effect of 1st mixing step compounds after annealing

Figure 7-12 shows the Payne effect value of the final step cured compounds in all polymer systems. Here, the advantage in the Si 263 compounds can be seen also in the NR or IR systems.
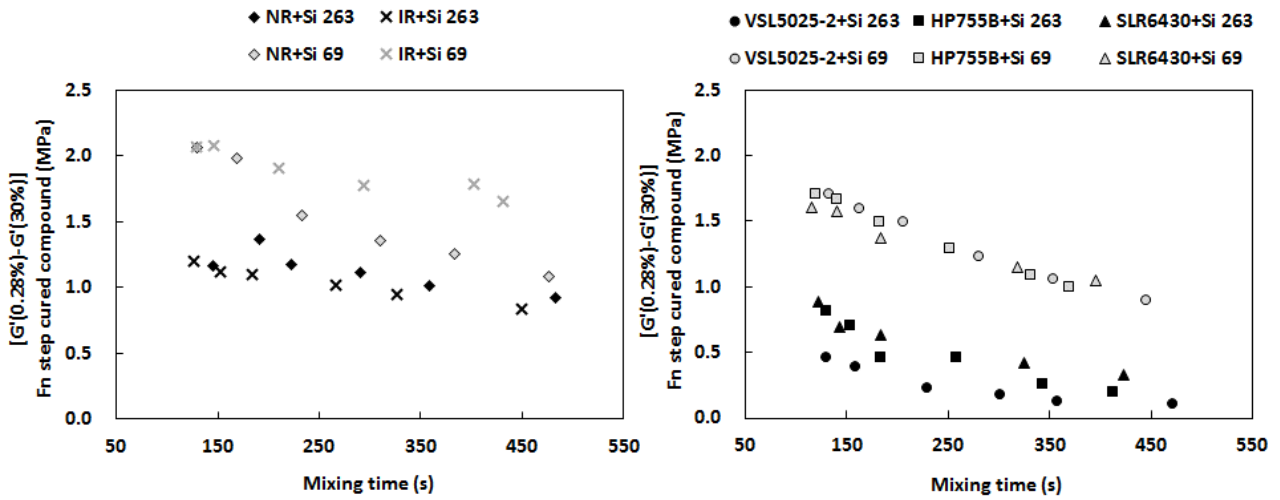

Figure 7-12: Payne effect values for the final step cured compounds as a function of the mixing time at the 1st mixing step 
However, even in the Si 263 compounds, the minimum Payne effect value of NR and IR systems is only on the same level as the value in the SBR / Si 69 systems. As it is possible to expect by the data of chemically bound rubber content, the silica flocculation is not suppressed to the same level as for the SBR / Si 263.

Regarding the Mooney viscosity values (Figure 7-13), although the values in the Si 263 compounds show slightly higher values than those in the Si 69 compounds at the 1st mixing stage, the tendency that the values increase with increasing mixing time shown in the SBR / Si 263 systems is not observed. Although the gap of the Mooney viscosity values between Si 263 and Si 69 compounds widens in the final mixing stage, the trend to increase the value with increasing mixing time is not observed (Figure 7-14). Interestingly, the phenomena that the overall bound rubber content at the final mixing stage decreases from those at the $1^{\text {st }}$ mixing stage is observed as shown in Figure 7-15, though the mechanism has not been clear yet. Consequently, the isoprene type of rubber / Si 263 compounds do not show the same level of silica flocculation suppression effect, but have the better processability compared to the SBR / Si 263 compounds.

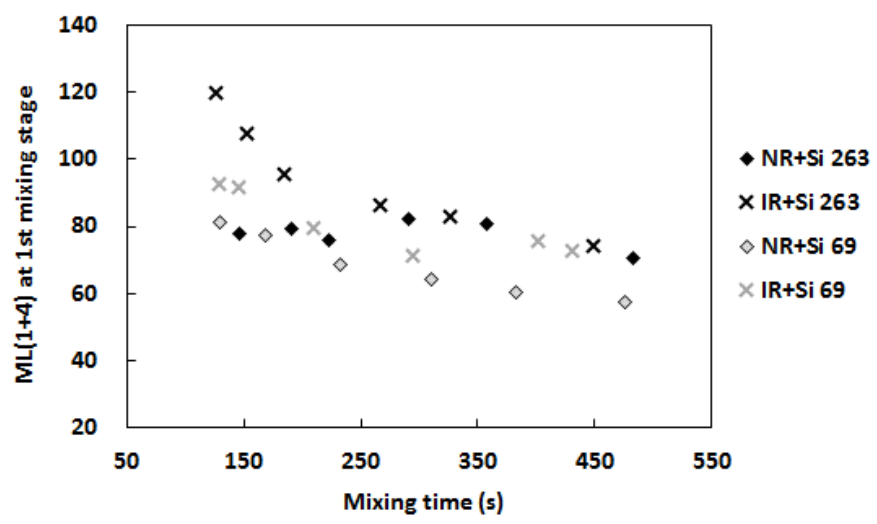

Figure 7-13: Mooney Viscosity of 1st mixing step compounds as a function of mixing time 


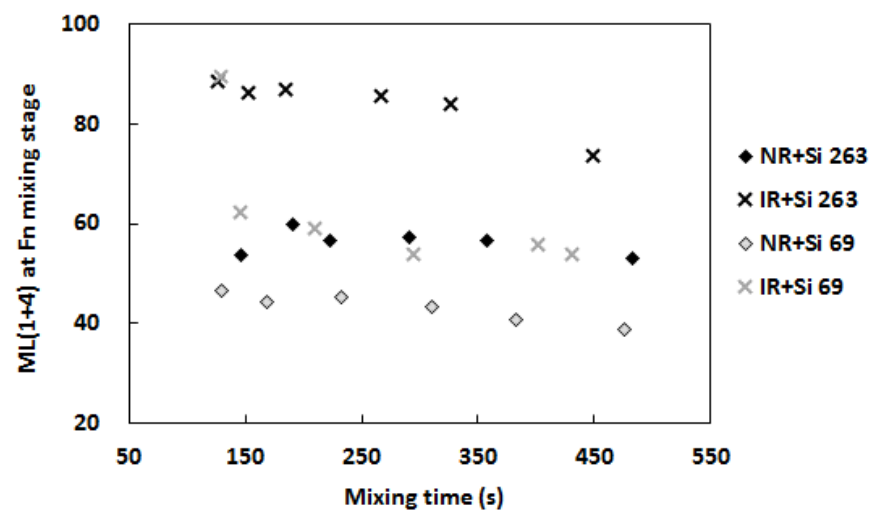

Figure 7-14: Mooney Viscosity of final mixing step compounds as a function of mixing time

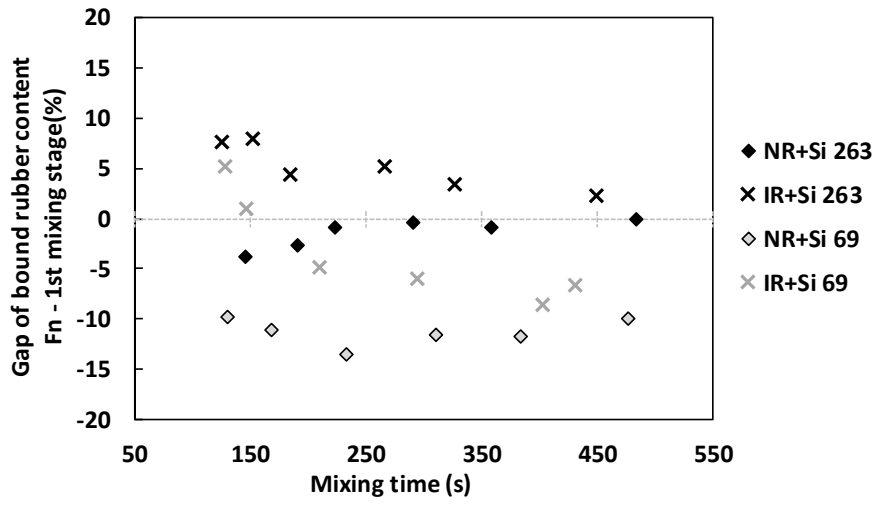

Figure 7-15: The gap of the overall bound rubber content between final mixing stage and $1^{\text {st }}$ mixing stage

\section{7-3-3 Curing behavior}

The cure characteristics of the compounds were measured to obtain the optimum vulcanization time for the measurements of mechanical and viscoelastic properties. However, the surprising phenomenon was observed only in the Si 263 systems that the torque did not rise up during the cure curve measurement at $160^{\circ} \mathrm{C}$, as shown in Figure 716. This was not observed in the SBR / Si 263 systems, while this occurs in all NR or IR / Si 263 compounds regardless the mixing time. 

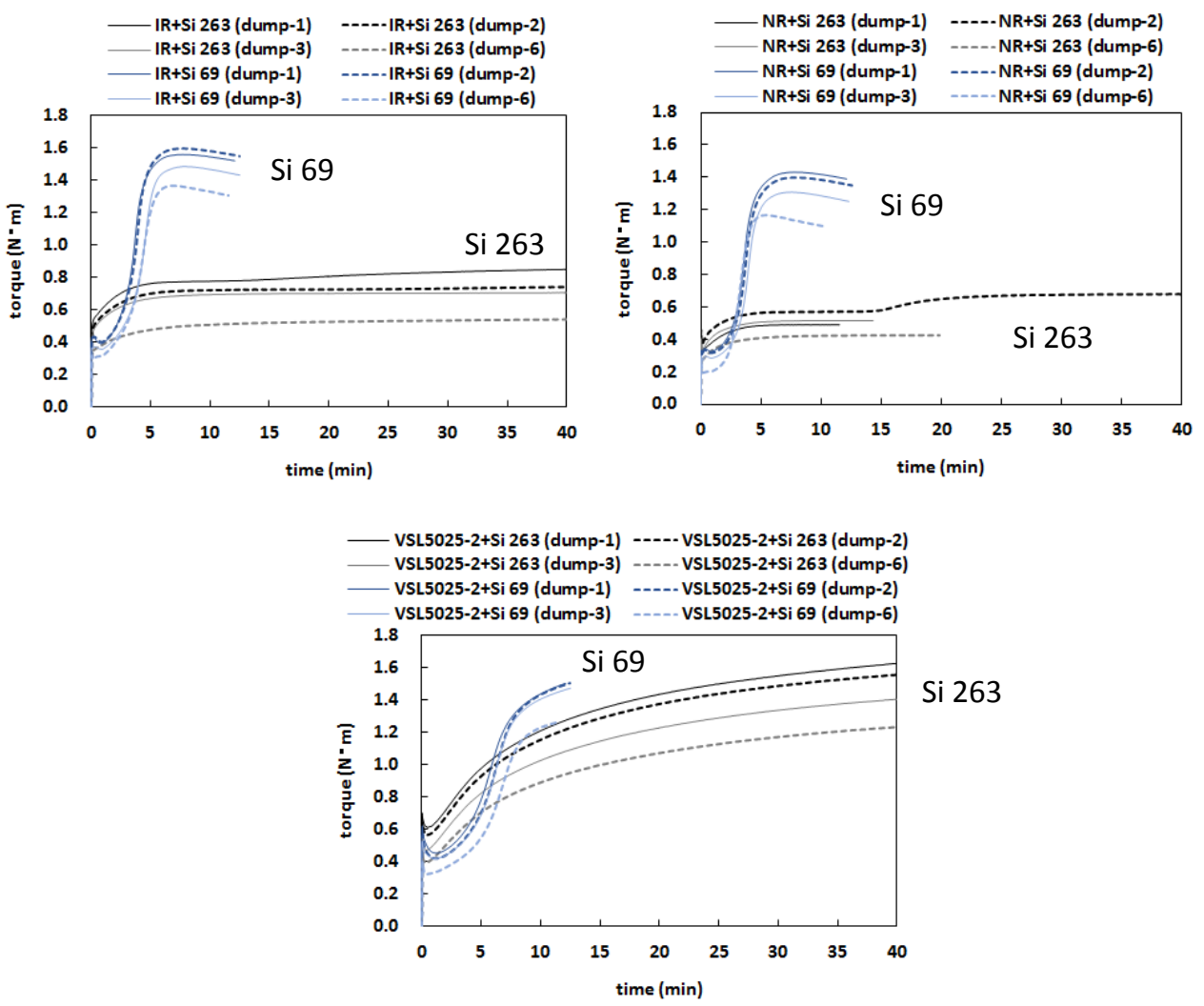

Si 263

Figure 7-16: Cure curves of the NR, IR or VSL5025-2 compounds

In table 7-4, the bonded sulfur content and the degree of swelling of the IR, VLS5025-2 and SLR6430 system's samples after conducting the normal vulcanization procedure at $160^{\circ} \mathrm{C}$ for $40 \mathrm{~min}$ are summarized. For comparison, the expected total sulfur content calculated from the formulation is also mentioned.

The adjustment of the net sulfur content between two silane systems was not carried out in this study. Hence, the total sulfur content in the Si 263 system is 0.23 wt.\% lower than that in the Si 69 system. Indeed, the measured bonded sulfur content in the IR / Si 263 systems is 0.34 wt.\% lower than that in the IR / Si 69 systems. This value is slightly higher than the calculated value from the formulation, however, exactly the same value is obtained in the VSL5025-2 systems. The actual bonded sulfur content is also quite similar in both IR or VSL5025-2 / Si 263 systems. Despite the facts, that the degree of swelling in the IR / Si 
263 system shows significantly higher values compared to other systems, which indicate that effective crosslinks were not formed in the IR / Si 263 system.

Since this tendency was observed not only in the NR / Si 263 system but as well in the IR / Si 263 system, the influence of the impurities inside the NR cannot be the reason for this unpredictable phenomenon. It is more likely that the combination of the isoprene micro structure and Si 263 leads to this peculiarity.

Table 7-4: Bonded sulfur content and degree of swelling

\begin{tabular}{|c|c|c|c|c|c|c|c|c|}
\hline \multirow{2}{*}{$\begin{array}{l}\text { IR system } \\
\text { Dump condition }\end{array}$} & \multicolumn{4}{|c|}{ Si 263 system } & \multicolumn{4}{|c|}{ Si 69 system } \\
\hline & $\begin{array}{c}150^{\circ} \mathrm{C} \\
\text { for } 3 \mathrm{~min}\end{array}$ & $150^{\circ} \mathrm{C}$ & $135^{\circ} \mathrm{C}$ & $120^{\circ} \mathrm{C}$ & $\begin{array}{c}150^{\circ} \mathrm{C} \\
\text { for } 3 \mathrm{~min}\end{array}$ & $150^{\circ} \mathrm{C}$ & $135^{\circ} \mathrm{C}$ & $120^{\circ} \mathrm{C}$ \\
\hline Bonded sulfur content (\%) & 1.01 & 0.98 & 1.00 & 1.01 & 1.33 & 1.37 & 1.33 & 1.34 \\
\hline Average of difference between two systems (\%) & \multicolumn{8}{|c|}{0.34} \\
\hline Degree of swelling (\%) & 329 & 333 & 352 & 375 & 221 & 200 & 196 & 190 \\
\hline VSL5025-2 / BR system & \multicolumn{4}{|c|}{ Si 263 system } & \multicolumn{4}{|c|}{ Si 69 system } \\
\hline Dump condition & \begin{tabular}{|c|}
$150^{\circ} \mathrm{C}$ \\
for $3 \mathrm{~min}$
\end{tabular} & $150^{\circ} \mathrm{C}$ & $135^{\circ} \mathrm{C}$ & $120^{\circ} \mathrm{C}$ & $\begin{array}{c}150^{\circ} \mathrm{C} \\
\text { for } 3 \mathrm{~min}\end{array}$ & $150^{\circ} \mathrm{C}$ & $135^{\circ} \mathrm{C}$ & $120^{\circ} \mathrm{C}$ \\
\hline Bonded sulfur content (\%) & 1.05 & 1.04 & 1.06 & 1.07 & 1.39 & 1.41 & 1.41 & 1.36 \\
\hline Average of difference between two systems (\%) & \multicolumn{8}{|c|}{0.34} \\
\hline Degree of swelling (\%) & 139 & 146 & 148 & 144 & 185 & 179 & 175 & 174 \\
\hline SLR6430 / BR system & \multicolumn{4}{|c|}{ Si 263 system } & \multicolumn{4}{|c|}{ Si 69 system } \\
\hline Dump condition & $\begin{array}{c}150^{\circ} \mathrm{C} \\
\text { for } 3 \mathrm{~min}\end{array}$ & $150^{\circ} \mathrm{C}$ & $135^{\circ} \mathrm{C}$ & $120^{\circ} \mathrm{C}$ & $\begin{array}{c}150^{\circ} \mathrm{C} \\
\text { for } 3 \mathrm{~min}\end{array}$ & $150^{\circ} \mathrm{C}$ & $135^{\circ} \mathrm{C}$ & $120^{\circ} \mathrm{C}$ \\
\hline Degree of swelling (\%) & 164 & 158 & 155 & 160 & 169 & 165 & 164 & 162 \\
\hline From formulation & \multicolumn{4}{|c|}{ Si 263 system } & \multicolumn{4}{|c|}{ Si 69 system } \\
\hline Total sulfur content (\%) & \multicolumn{4}{|c|}{1.15} & \multicolumn{4}{|c|}{1.38} \\
\hline Difference between two systems (\%) & \multicolumn{8}{|c|}{0.23} \\
\hline
\end{tabular}

The influence of the cure package was checked simply by changing it as shown in Table 7-5, however, rising up the torque in the cure curve could again not be obtained.

Table 7-5: Tested new cure package including TBzTD (tetrabenzylthiuram disulfide)

\begin{tabular}{|c|c|c|}
\hline & $\begin{array}{c}\text { cure system-1 } \\
\text { (phr) }\end{array}$ & $\begin{array}{c}\text { cure system-2 } \\
\text { (phr) }\end{array}$ \\
\hline sulfur & 1.50 & 2.00 \\
CBS & 1.70 & 2.00 \\
TBzTD & - & 1.00 \\
\hline
\end{tabular}

Based on the consideration so far, the following seems to be suggested as the reason; 
1. Polymer degradation occurred significantly resulting in losing the crosslinking efficiency. 2. Significant amount of mercapto-silanes were transformed into a disulfide-silane, and they are coupled to the silica surface.

3. Curatives can create a lot of pendant and / or cyclic sulfur groups.

In order to confirm the first idea, the molecular weight of sol fraction, which means the soluble part in THF after the $1^{\text {st }}$ mixing step, was measured. The results are shown in Figure 7-17. The molecular weight of the sol fraction in the IR / Si 263 compounds aligns on the same line as that in the IR / Si 69 compounds. And, the absolute value of the molecular weight is higher than the value of the SBR systems. Therefore, it is reasonable to assume that the rubber matrix still has a sufficient level of molecular weight in order to create effective crosslinkings in the IR / Si 263 systems. This leads to the conclusion, that an excess degradation of polymers in the system cannot be the reason for not rising up the torque in the rheogram.

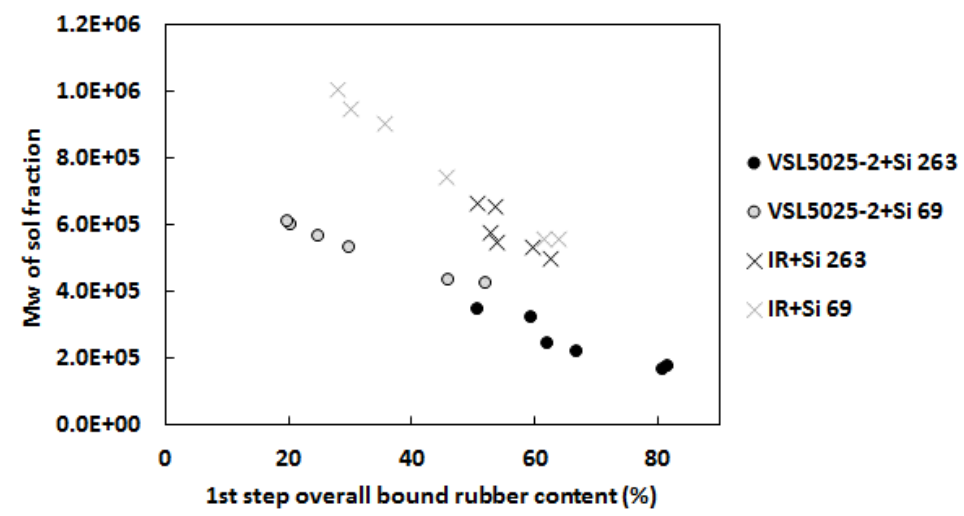

Figure 7-17: Molecular weight of sol fraction after 1st mixing step samples

The mechanism of the second possibility is as following; The mercapto-silane radicals can undergo the addition reaction to the isoprene type of double bonds to a certain degree, however, as the reaction is a reversible one, the resulting amount of the addition reaction product could be small. As a result, large amounts of mercapto-silane radicals can be present in the system, which subsequently combines together to become disulfide-silanes. As the isolated disulfide-silanes can be extracted by acetone resulting in the lower bonded sulfur content, those disulfide-silanes should be coupled to the silica surface. If silanes have remained as disulfide-silanes, the elemental sulfur added at the final mixing stage can be 
used to be incorporated inside the S-S bond in the silanes. Consequently, the remaining amount of elemental sulfur which can be used for the matrix crosslinking becomes less. Then, because of no DPG in the system, the vulcanization rate could be very slow. However, it seems that this mechanism may not be true because the problem could not be solved also in the cure system-2 shown in Table 7-5, which seems to have a reasonable cure rate due to the additional presence of TBzTD.

After all, the exact mechanism for this phenomena has not been clarified yet, therefore, further experiments such as the crosslink structure analysis including the pendant and cyclic structures are necessary.

\section{7-4 Conclusions}

The NR or IR / Si 263 or Si 69 compounds were mixed with six different mixing times and compared with the SBR / BR blend compounds.

The observed same tendencies as in the SBR-BR compounds were that the Si 263 compounds created the higher chemically bound rubber from the short mixing time and the polymer network had denser structure compared to the Si 69 compounds. However, the overall bound rubber contents in both silane systems came to a similar level when the mixing time reaches at $250 \mathrm{~s}$ and then kept on the same level. Furthermore, the maximum bound rubber content in the NR or IR compounds were limited to the same level as that in the SBR-BR / Si 69 compounds and did not reach the maximum value which is achieved in the SBR-BR / Si 263 compounds. The polymer network of the chemically bound rubber inside the NR or IR / Si 263 compounds showed a denser structure as that of all Si 69 compounds including the SBR-BR compounds, but was less dense structure compared to that in the SBR-BR / Si 263 compounds.

The addition reactivity of mercapto-silane radicals to the isoprene type of double bonds seems to be lower than that to the vinyl and cis double bonds in the butadiene structure. This can be considered as one of the main reasons for a lower maximum bound rubber content in the NR or IR / Si 263 compounds compared to the SBR-BR / Si 263 compounds. However, considering the fact that the Si 263 system showed higher chemically bound 
rubber content than the Si 69 compounds at the short mixing time, certain amount of the addition reaction seemed to occur also in the NR or IR compounds. In the case of the isoprene type of polymers, the allylic hydrogen abstraction and the subsequent delocalization effect of the radical lead to the creation of the tertiary radicals which have a relatively higher stability. These radicals are known to prefer the coupling reaction between the radicals to the radical chain reaction with the other double bonds, thus, this difference also seemed to be the main reason for reaching a lower chemically bound rubber content compared to the SBR systems. Additionally, the easiness of the main chain scission in the isoprene type of rubber could also be the reason for not showing the drastically increase of the bound rubber content with increasing mixing time. This influence should be more pronounced in the Si 263 compounds because of the potential to create the larger amount of polymer radicals.

The values of the Payne effect before and after annealing at $170{ }^{\circ} \mathrm{C}$ indicated that the NR or IR / Si 263 compounds have the higher silica flocculation suppression effect compared to the Si 69 compounds, though the degree was not as high as that in the SBR-BR / Si 263 compounds. As there was almost no difference regarding the bound rubber content between silanes in the longer mixing time compounds, the higher effect for the flocculation suppression seemed to be because of the slightly denser bound rubber network together with the shielding effect on the silica surface brought by the polymers which are directly bonded to the silica via silane. On one hand, the degree to hinder the silica flocculation is not high as that in the SBR-BR / Si 263 compounds, on the other hand, the tendency that the Mooney viscosity increases with increasing mixing time was not observed and thus the processaiblity of the compounds seemed to be better than that in the SBR-BR / Si 263 compounds.

When checking the cure characteristics, the problem that the torque did not rise up during the cure curve measurement at $160^{\circ} \mathrm{C}$ in the NR or IR / Si 263 compounds came into focus. The sulfur content measurement of the residue samples after the extraction by acetone showed similar values as those in the SBR-BR compounds, therefore, it can be assumed that the curatives have been bonded to the polymer or the silica, but cannot create the effective crosslinking in the rubber matrix. The exact mechanism has not been clarified yet, therefore, further experiments are necessary. 
Overall, it was revealed that the combination with micro structure of the polymer significantly influences the internal reinforcing structure in the mercapto-silane systems. The usage of the mercapto-silane together with the isoprene type of polymers did not leads to the same level of the silica dispersion state after the vulcanization step as the butadiene type of polymers, though the processability seemed to be better. Moreover, the combination of the isoprene type of polymers and silanes which could generate a large amount of polymer radicals such as mercapto-silanes cannot avoid the degradation of the polymer, and the longer mixing time at higher temperature in order to boost the silanization reaction is also not desirable for these polymers. Therefore, it is concluded that the development of a coupling agent which can combine the silica and the polymer without causing degradation of the polymer is necessary. 


\section{7-5 References}

[1] S. Cayeux, L. Guy, C. Boivin, T. Chaussée et al, Tire Tech 2017, Hannover, February 14-16 (2017)

[2] S.S. Sarkawai, W.K. Dierkes, J.W.M. Noordermeer, European Polymer Journal, 49 (2013) 3199-3209

[3] K. Nawamawat, J. Sakdapipanich, D. Mekkriengkrai, Y. Tanaka, Kautsch. Gummi Kunstst., $61(2008) 518$

[4] L. Tarachiwin, J. Sakdapipanich, K. Ute, T. Kitayama, T. Bamba, E. Fukusaki, A. Kobayashi, Y. Tanaka, Biomacromolecules., 6 (2005) 1851

[5] S. Amnuaypornsri, J. Sakdapipanich, S. Toki, B.S. Hsiao, N. Ichikawa, Y. Tanaka, Rubber Chem. Technol., 81 (2008) 753-766

[6] K. Nawamawat, J. Sakdapipanich, D. Mekkriengkrai, Y. Tanaka, Kautsch. Gummi Kunstst., (2008) 518-522

[7] J. Kruzelak, R. Sykora, I. Hdec, Rubber Chem. Technol., 90 (2017) 60-88

[8] M. Akiba, A. S. Hashim, Prog. Polym. Sci., 22 (1997) 475-521

[9] S. Yamashita, Journal of the Society of Rubber Science and Technology, 76 (2003) 186191

[10] S. Yamashita, Journal of the Society of Rubber Science and Technology, 41 (1968) 380399

[11] L. Gonza'lez, A. Rodr'ıguez, A. Marcos, C. Chamorro, Rubber Chem. Technol., 69 (1996) 203-214

[12] L. Gonzalez, A. Rodriguez, J. LValentin, A. Marcos-Fernandez, P. Posads, Kautsch. Gummi Kunstst., 58 (2005) 638-643

[13] P. Gosh, S. Katare, P. Palkar, J.M. Carethers, V. Venkatasuramaniam, K.A. Walker, Rubber Chem. Technol., 76 (2003) 592-693

[14] J.L. Valentin, P. Posadas, A. Fernandez-Torres, M.A. Malmierca, L. Gonzalez, W. Chasse, K. Saalwachter, Macromolecule, 43 (2010) 4210-4222 


\section{Chapter 8}

\section{Summary}

Tire performances have to be improved further in order to fulfill the increasing requirements. Low rolling resistance tires are essential for the reduction of fuel consumption resulting in the contribution of a preservation of petroleum resources, and even for a reduction of the $\mathrm{CO}_{2}$ emission. At the same time, the higher wet grip performance has to be obtained from the safety point of view with maintaining the abrasion resistance. Over the last few decades, the silica / silane technology has become standard in the tire industry as an effective approach to improve these tire properties. The usage of a bifunctional silane coupling agent enables a good dispersion of the polar silica in the nonpolar rubber matrix by hydrophobizing the surface. Furthermore, a sufficient reinforcing effect is established by directly coupling from the silane-modified silica to the rubber. The usage of higher surface area silica seems to have a further potential to increase the achievable performance balance between the required low rolling resistance, the high wet grip performance and the high abrasion resistance because of the higher reinforcing potential of it. But, the higher the surface area of silica is, the more difficult it is to disperse this silica in the rubber matrix because of the higher self-cohesive forces. To overcome this point, the use of mercapto-silanes has become more and more into focus over the last years. The use of them leads to an increase of the silica dispersion level compared to the conventional sulfide-silanes. However, they cause also processability problems such as a high Mooney viscosity and a premature scorch. The mechanism behind them has not been clarified yet.

Therefore, the first objective of the present thesis was set on the understanding of the reactivity and the reaction mechanism of mercapto-silanes with rubber in comparison with those of traditionally used sulfide-silanes. Additionally, the relationship between the resulting reinforcing structure and the physical properties of these rubber compounds was in focus of this study. The reactivity and the mechanism between a silane and a polymer are expected to vary depending on the structure of both reactants. Therefore, various combinations between different structural varied (model) polymers and silanes are 
investigated. These evaluations were expected to be the basis to find a solution to overcome the limitations in the use of mercapto-silanes in tire tread compounds as well as to evaluate the ideal internal structure to balance all properties including the processability on the highest possible level.

For a good dispersion of silica into a rubber matrix resulting in an effective reinforcement, the characteristics of the silica itself, the silanization reaction and the coupling reaction of silane with rubber play an important role. To establish a good basis for all planned investigations a detailed literature study about these points was carried out (Chapter 2). The evaluation methods of the resulting reinforcing chemical structures in the compound and the relationship of them with the physical properties were reviewed as well. Moreover, based on the fact that the silane - polymer reactions are sulfur related reactions, the sulfur vulcanization mechanisms and the representative model studies to clarify the mechanism were also summarized.

The experimental part was divided into two parts: the investigation of the silane-polymer coupling in a model study and in a real rubber compound. In the model study, the reactions of a mercapto-silane (Si 263) or a sulfide-silane (Si 69) with various types of double bond structures were investigated by using six different model olefins (Chapter 3 ). The model olefin studies are effective to elucidate the reactivity and the exact coupling mechanism because the study can be carried out in solution which enables the use of different analytical methods. Furthermore, the structure of the reaction products is less complex compared to the long polymer molecules. As a result of this study, it was confirmed that the above mentioned silanes following different mechanisms in their reaction with the used olefins. The preferred reaction depends on the olefin structure even when the same silane is used. Mercapto-silane radicals were generated without adding a radical initiator which results partly in an addition reaction to the double bond of the olefin. The addition reaction products were obtained by using 3-methyl-1-pentene $(3 \mathrm{~m} 1 \mathrm{p})$, which has a vinyl double bond, in both cis and trans-3-hexenes $(\mathrm{C} 3 \mathrm{H}$ and $\mathrm{T} 3 \mathrm{H})$ and in trans-3-methyl-3-hexene (T3M3H) systems, however, the detectable amounts were very different in each system. The highest amount was obtained with the vinyl structure $(3 \mathrm{~m} 1 \mathrm{p})$, but only very small amounts with the isoprene double bond in the T3M3H system. Additionally, large amounts of isomers of the olefin were detected in the $\mathrm{C} 3 \mathrm{H}$ and $\mathrm{T3M} 3 \mathrm{H}$ systems. As the addition reaction of thiol radicals to double bonds is known to be a reversible reaction, isomers can be produced during this process. In addition, as double bond shifted isomers were also 
obtained, the abstraction of the allylic hydrogen and the subsequent radical delocalization can also be considered as the explanation for the observed isomerization process. The easiness of the addition reaction depends on the stability of the double bond of the olefin. The resulting ratio of the addition reaction product to the original olefin and the isomers seems to be determined by the stability balance of those structures. It is considered that when the stability of the double bond in the original olefin or the generated radical by the allylic hydrogen abstraction was high, the abstraction could easily and with higher priority occur compared to the addition reaction.

Similar to the Si 263 system, an isomerization was observed in the Si 69 / C3H or T3M3H system. However, the isomerization rate was slower and the number of isomers was lower than those in the Si 263 systems. This indicated that the ability of the addition reaction as well as the allylic hydrogen abstraction from double bonds were much lower inside the Si 69 system compared to those in the Si 263 system. Therefore, it can be assumed that Si 69 has a lower ability to create reactive radical species from olefins compared to $\mathrm{Si} 263$. As another characteristic in the Si 69 / tetra-methyl-ethylene (TME) system, a cross-linked product where two molecules of olefin were combined by sulfur coupled the allylic carbons was observed. This sulfur was released from the Si 69 molecule which acted as a sulfur donor. Furthermore, a decrease of the double bond after heating was observed in the $\mathrm{C} 3 \mathrm{H}$ and $\mathrm{T} 3 \mathrm{M} 3 \mathrm{H} / \mathrm{Si} 69$ systems. In this case, a sulfur containing structure might be considered as the resulting structure.

As explained so far, it was clarified that the influencing mechanism of mercapto-silanes and sulfide-silanes on olefins are completely different. The amount of the silane fragment directly added to an olefin and also the generated olefin radicals seem to be considerably higher in the Si 263 system compared to those in the Si 69 system.

The influence of further ingredients like silica, $\mathrm{ZnO}, \mathrm{CBS}$ and sulfur on the addition reaction was investigated using the $3 \mathrm{~m} 1 \mathrm{p} / \mathrm{Si} 263$ system in Chapter 4. It was confirmed that the observed direct addition reaction product between 3m1p and Si 263 in Chapter 3 is also produced in the presence of silica and that most of it is coupled to the silica surface as well. The addition of $\mathrm{ZnO}, \mathrm{CBS}$ or sulfur suppresses the addition reaction between $3 \mathrm{~m} 1 \mathrm{p}$ and Si 263. Instead of that, large amounts of disulfide-silanes (also trisulfide-silanes in the case of sulfur adding system) were detected as a conversion from Si 263 . This indicates that the presence of these ingredients changes the preferred reaction of mercapto-silane from 
the radical addition reaction to the coupling reaction between two silanes. Transferring this knowledge to the rubber system, the addition of these ingredients could lead to the suppression of the reaction between rubber and mercapto-silane. If the reason for worse processability in the mercapto-silane system is the excess reaction between the mercaptosilane and the rubber, these ingredients might have the possibility to overcome this.

After that, the model study was varied by using squalene or liquid butadiene rubbers as model substances (Chapter 5). As they have more than one double bond in a molecule and a higher molecular weight, the systems should simulate the phenomena in the actual tire tread compound more precisely compared to the case by using model olefins. The observed tendencies are basically in a good agreement with the results of the olefin study. The observed isomerization degree in the Si 263 systems was higher than that in the Si 69 systems. Furthermore, the addition product of Si 263 to the vinyl and cis double bonds in liquid butadiene rubbers and also to squalene were detected, though the amount in the squalene system was considerably lower. In the Si 69 systems, the isomerization amount was low and possible addition products were not detected. However, a decrease of the amount of double bonds were observed in all Si 69 systems, and as the ${ }^{13} \mathrm{C}$ NMR spectrum in the squalene / Si 69 sample after heating shows a very similar signal pattern as the one observed in the squalene / sulfur system without any accelerators after heating, the formation of cross-linked products also in the Si 69 system was strongly indicated. This corresponds to the achieved result in the model olefin TME system. Therefore, it can be concluded that Si 69 has been acting as a sulfur donor without additional sulfur or accelerators, as already reported in many papers.

All results of the model study were now used as a good basis for further investigations in the rubber compound. In Chapter 6, the mixing of the typical passenger car tire tread compounds was carried out which contains SBR / BR blend silica filled compounds in combination with a mercapto-silane or a sulfide-silane. The obtained internal structure and compound properties were explained based on the findings in the model study. The Si 263 compounds create larger amounts of chemically bound rubber even with shorter mixing time compared to the Si 69 compounds. In addition, the chemically bound rubber in the Si 263 compound was determined by swelling measurements and indicates a significantly denser polymer network. As already observed in the model study, the very high reactivity of the mercapto-silane radical to the rubber polymer double bonds, especially to vinyl and cis double bonds, increases the number of the coupling points between silica and rubber 
via silane. This is supported by the high radical chain reactivity of SBR polymer radicals generated by the reversible addition reaction and the abstraction of the allylic hydrogen by the mercapto-silane radicals and the intermediate (thioether and carbon centered) radicals. The continuation of the radical chain reaction leads to the creation of large amounts of $\mathrm{C}-\mathrm{C}$ bonds resulting in a dense polymer network.

The Si 263 compounds provided at the same mixing time lower tan $\delta$ at $60^{\circ} \mathrm{C}$ compared to the Si 69 compounds. Additionally, the values seem to correlate with the Payne effect values of the cured compounds. By the flocculation measurements of the uncured compounds, it was confirmed that the Si 263 compounds flocculate less, which results in lower Payne effect values as well as lower tan $\delta$ values at $60^{\circ} \mathrm{C}$ after the vulcanization. This difference seems to be because of the large amount of chemically bound rubber content together with its dense and developed structure in the mercapto-silane compounds. The bound rubber shields the silica surface, moreover, the formed dense and developed structure reduces the reagglomeration tendency of silica clusters. The suppression of the silica flocculation was observed most clearly in the high-vinyl type of SBR / Si 263 system. This seems to be because of the largest amount of bonding points between silica and polymer via silane. However, in all SBR / Si 263 systems, the increase of the Mooney viscosity or the tendency to worsen the dump compound appearance with increasing mixing time was observed. This indicates that the large amounts of chemical linkages between polymer and silica via silane in combination with the developed dense bound rubber networks cause a lower flexibility of the uncured compounds, which leads to the observed worse processability.

Taken as a whole, increasing the direct bonding between silica and polymer via silane in order to obtain a sufficient shielding effect by polymer on the silica surface together with preventing the formation of a too dense bound rubber network caused by the excess amount of $\mathrm{C}-\mathrm{C}$ bonding seem to be the better direction to balance the silica dispersion and the processability.

In Chapter 7, the mixing of silica filled NR or IR compounds was carried out. The results were compared to those from chapter 6, using SBR / BR blends. Although the higher chemically bound rubber content was obtained in the Si 263 compounds which is the same observation as in the SBR / BR system, the maximum bound rubber content in the NR or IR / silane compounds were limited to the same level as that in the SBR-BR / Si 69 compounds 
and did not reach the maximum value which was achieved in the SBR-BR / Si 263 compounds. The polymer network of the chemically bound rubber inside the NR or IR / Si 263 compounds shows a denser structure as that of all Si 69 compounds including the SBR / BR compounds, but is less dense compared to that in the SBR-BR / Si 263 compounds. The lower addition reactivity of mercapto-silane radicals to the isoprene type of double bonds together with the preferred coupling reaction between tertiary radicals, which can be created by the allylic hydrogen abstraction and the subsequent delocalization effect, seem to be the main reasons for reaching a lower chemically bound rubber content compared to the SBR systems. Additionally, the easiness of the main chain scission in the isoprene type of rubber could also be the reason for not showing the drastically increase of the bound rubber content with increasing mixing time.

The values of the Payne effect before and after annealing at $170{ }^{\circ} \mathrm{C}$ indicated that the NR or IR / Si 263 compounds have the higher silica flocculation suppression effect compared to the Si 69 compounds, though the degree was not as high as that in the SBR-BR / Si 263 compounds. As there was almost no difference regarding the bound rubber content between silanes in the longer mixing time compounds, the higher effect for the flocculation suppression seems to be because of the slightly denser bound rubber network together with the shielding effect on the silica surface brought by the polymers which are directly bonded to the silica via silane. On one hand, the degree to hinder the silica flocculation is not as high as that in the SBR-BR / Si 263 compounds, on the other hand, the tendency that the Mooney viscosity increases with increasing mixing time was not observed and thus the processability of the compounds seems to be better than that in the SBR-BR / Si 263 compounds.

Overall, it was revealed that the micro structure of the polymer significantly influences the internal reinforcing structure in the mercapto-silane systems. The usage of the mercapto-silane together with the isoprene type of polymers did not lead to the same level of silica dispersion after the vulcanization step as the butadiene type of polymers, though the processability seems to be improved. Moreover, the combination of the isoprene type of polymers and silanes which could generate a large amount of polymer radicals such as mercapto-silanes cannot avoid the degradation of the polymer. Additionally, the longer mixing time at higher temperature in order to boost the silanization reaction is also not desirable for these polymers. Therefore, it is concluded that the development of a coupling 
agent which can combine the silica and the polymer without causing degradation of the polymer is necessary.

The final conclusions are, although silica filled compound technologies have been global standard for passenger tire tread compounds nowadays, further performance improvements have always been demanded. To improve a dispersion level of higher surface area of silica, which is generally more difficult to disperse into the rubber matrix, seems to be one of the positive directions to increase the reinforcement level with decreasing the possible energy dissipation during a rotation of the tire. In the present thesis, by investigating the reaction mechanism of sulfide-silane and mercapto-silane the reinforcing structure which realizes a lower filler-filler interaction after vulcanization was clarified. Mainly two points seem to be the reason for enabling mercapto-silanes to achieve the lower filler-filler interaction. The first one is a higher shielding effect by a polymer attached to the silica surface, due to the higher addition reaction ability of mercapto-silanes to the double bond. The second point is the formation of a dense bound rubber structure by the radical propagation reaction between polymers started by the polymer carbon radical induced by the mercapto-silane radical. These structures suppress the silica flocculation during the vulcanization stage, resulting in the observed lower Payne effect values. However, as the effectiveness of mercapto-silanes on the above mentioned two points depends on the double bond structure of the polymer, mercapto-silanes are not applicable to all types of polymers. Furthermore, especially the second point can additionally explain the observed worse processability by the existence of the above mentioned dense polymer network already in the uncured compound. Further studies are still necessary to find the optimum way to balance the better silica dispersion level and the processability regardless of the polymer type. However, the findings presented in this thesis provide many crucial hints how to control during mixing the reactions which should occur and how to suppress the unwanted side reactions. The control could be done by the structure improvement of silanes and / or polymers or by third additives which have specific functions. The results in this thesis are expected to deliver a valuable contribution to find the optimum control which could lead to the next technical innovations in the tire industry. 


\section{Samenvatting}

De eigenschappen van banden moeten verder worden verbeterd om aan toenemende eisen te blijven voldoen. Lage rolweerstand banden zijn essentieel voor de vermindering van brandstofverbruik, resulterend in een bijdrage aan het behoud van olie-reserves en zelfs aan vermindering van $\mathrm{CO}-2$-emissie. Tegelijkertijd moet een hogere natte grip worden verkregen vanuit een veiligheids-standpunt bij handhaving van de slijtage weerstand. Gedurende de afgelopen decennia is de silica/silaan technologie de standaard geworden in de bandenindustrie als effectieve benadering om deze bandeigenschappen te verbeteren. Het gebruik van een bi-functioneel silaan koppelings-middel geeft een goede verdeling van het polaire silica in de niet-polaire rubber-matrix door hydrofobering van het oppervlak. Verder wordt een voldoende versterkend effect bereikt door directe koppeling van het silaan-gemodificeerde silica aan de rubber. Het gebruik van hogere specifieke oppervlakte silica schijnt een verdergaande mogelijkheid te bieden om de bereikbare functionele balans tussen de vereiste rolweerstand, de hoge natte grip en hoge slijtageweerstand te verbeteren vanwege het hogere versterkings-vermogen daarvan. Evenwel, hoe hoger het oppervlak van de silica, des te moeilijker is het om de silica in de rubber-matrix te dispergeren vanwege de hogere samenhangende krachten. Om dit te verhelpen is het gebruik van mercapto-silanen meer en meer in beeld gekomen over de afgelopen jaren. Gebruik daarvan leidt tot een toename van het silica dispersie niveau vergeleken met de conventionele sulfide-silanen. Zij veroorzaken echter ook verwerkingsproblemen, zoals een hoge Mooney viscositeit en vroegtijdige aanvulkanisatie (scorch). Het mechanisme daarachter is nog niet opgelost.

Daarom was als eerste doelstelling van het huidige proefschrift gesteld het begrijpen van de reactiviteit en het reactie-mechanisme van mercapto-silanen met rubber in vergelijking met die van gebruikelijke sulfide-silanen. Verder was de relatie tussen de resulterende versterkende structuur en de fysische eigenschappen van deze rubber compounds een punt van aandacht in deze studie. De reactiviteit en het mechanisme tussen een silaan en een polymeer worden verwacht te verschillen afhankelijk van de structuur van beide reactanten. Daarom worden verschillende combinaties tussen uiteenlopende, qua structuur gevarieerde (model) polymeren en silanen onderzocht. Deze evaluaties werden verwacht als basis te kunnen dienen voor het vinden van een oplossing voor de beperkingen in het 
gebruik van mercapto-silanen in banden-loopvlak mengsels. En daarnaast om de ideale interne structuur te evalueren voor het in evenwicht brengen van alle eigenschappen op het hoogst mogelijke niveau met inbegrip van verwerking.

Voor een goede verdeling van silica in een rubber matrix resulterend in effectieve versterking spelen de karakteristieken van de silica zelf, de silaniserings-reactie en de koppelings-reactie van silaan met rubber een belangrijke rol. Om een goede basis te creëren voor alle geplande onderzoeken is een gedetailleerde literatuurstudie rond deze punten uitgevoerd: Hoofdstuk 2. De evaluatie-methoden van de resulterende versterkende chemische structuren in het compound en de relatie daarvan met de fysische eigenschappen werden eveneens bekeken. Verder, gebaseerd op het feit dat de silaanpolymeer reacties zwavel-gerelateerd zijn, zijn de zwavel vulcanisatie mechanismen en representatieve model-studies om het mechanisme op te helderen samengevat.

Het experimentele deel is verdeeld in twee delen: het onderzoek van de silaan-polymeer koppeling in een model-studie en in een werkelijk rubber mengsel. In de model-studie zijn de reacties onderzocht van een mercapto-silaan (Si 263) of een sulfide-silaan (Si 69) met verschillende types dubbele binding structuren door gebruikmaking van zes verschillende model-olefinen: Hoofdstuk 3. De model-olefine studies zijn in staat de reactiviteit en het exacte koppelings-mechanisme op te helderen, omdat de studie in oplossing kan worden uitgevoerd, hetgeen de toepassing van verschillende analytische methodes mogelijk maakt. Bovendien is de structuur van de reactieproducten minder ingewikkeld vergeleken met de lange polymeer-moleculen. Als resultaat van deze studie werd bevestigd, dat de boven vermelde silanen verschillende mechanismen volgen in hun reacties met de gebruikte olefinen. De gewenste reactie hangt van de olefine-structuur af, zelfs wanneer dezelfde silaan wordt gebruikt. Mercapto-silaan radicalen werden gevormd zonder dat een radicaalinitiator was toegevoegd, hetgeen gedeeltelijk resulteert in een additie-reactie aan de dubbele binding van het olefine. De additie reactie-producten werden verkregen bij gebruikmaking van 3-methyl-1-penteen ( $3 \mathrm{~m} 1 \mathrm{p})$, wat een vinyl dubbele band heeft, in zowel cis- en trans-hexenen ( $\mathrm{C} 3 \mathrm{H}$ en $\mathrm{T} 3 \mathrm{H})$ en in trans-3-methyl-3-hexeen (T3M3H) systemen. Echter de detecteerbare hoeveelheden verschilden sterk in elk systeem. De hoogste hoeveelheid werd verkregen met de vinyl-structuur $(3 \mathrm{~m} 1 \mathrm{p})$, maar slechts heel lage hoeveelheden bij gebruik van de isopreen dubbele-binding in het $\mathrm{T} 3 \mathrm{M} 3 \mathrm{H}$ systeem. Daarbovenop werden grote hoeveelheden isomeren gedetecteerd van het olefine in de $\mathrm{C} 3 \mathrm{H}$ en $\mathrm{T3M} 3 \mathrm{H}$ systemen. Aangezien de additie-reactie van thiol-radicalen aan dubbele 
bindingen bekend is reversibel te zijn, kunnen isomeren worden geproduceerd gedurende dit proces. Bovendien, omdat isomeren met verschoven dubbele band ook werden verkregen, kan abstractie van allylische waterstof en de daaropvolgende radicalaire delocalisatie ook worden beschouwd als verklaring voor het waargenomen isomerisatie proces. Het gemak van de additie-reactie hangt af van de stabiliteit van de dubbele binding van het olefine. De resulterende verhouding van het additie reactie-product van het oorspronkelijke olefine ten opzichte van de isomeren schijnt te worden bepaald door de stabiliteits-balans van deze structuren. Er wordt in aanmerking genomen, dat wanneer de stabiliteit van de dubbele band in het oorspronkelijke olefine of van het gevormde radicaal door de allylische waterstof-abstractie hoog was, de abstractie gemakkelijk en met hoge prioriteit kon plaatsvinden vergeleken met de additie-reactie.

Vergelijkbaar met het Si 263 systeem werd isomerisatie waargenomen in het Si 69/C3H of T3M3H systeem. Echter de isomerisatie-snelheid was langzamer en het aantal isomeren lager dan in de Si 263 systemen. Dit geeft aan dat de reactiviteit van de additie-reactie en de allylische waterstof-abstractie van dubbele bindingen veel lager waren in het Si 69systeem vergeleken met het Si 263-systeem. Daarom kan worden aangenomen, dat Si 69 een lager vermogen bezit om reactieve radicaal-soorten te vormen vergeleken met Si 263. Als andere karakteristiek in het Si 69/tetra-methyl-etheen (TME) systeem werd een vernet product waargenomen, waarin twee olefine-moleculen waren gecombineerd door zwavel op de allylische koolstoffen. Deze zwavel was vrijgekomen uit het Si 69 molecuul dat als zwavel-donor fungeerde. Bovendien werd een afname waargenomen van de dubbele binding na verhitting in de $\mathrm{C} 3 \mathrm{H}$ en $\mathrm{T} 3 \mathrm{M} 3 \mathrm{H} / \mathrm{Si} 69$ systemen. In dit geval zou een zwavelbevattende structuur kunnen worden opgevat als resulterend.

Zoals tot nu toe uitgelegd, was het duidelijk dat de beïnvloedende mechanismen van mercapto-silanen en sulfide-silanen op olefinen volledig verschillend zijn. De hoeveelheden silaan-fragmenten direct toegevoegd aan een olefine en ook de gevormde olefine-radicalen schijnen aanzienlijk hoger te zijn in het Si 263 systeem vergeleken met die in het Si 69 systeem.

De invloed van overige ingrediënten zoals silica, $\mathrm{ZnO}, \mathrm{CBS}$ en zwavel op de additie-reactie werd onderzocht met gebruik van 3m1p/Si 263 in Hoofdstuk 4. Er werd bevestigd, dat het in Hoofdstuk 3 waargenomen directe additie reactie-product tussen $3 \mathrm{~m} 1 \mathrm{p}$ en Si 263 ook wordt gevormd in aanwezigheid van silica en dat het meeste daarvan ook wordt gekoppeld 
aan het silica-oppervlak. De toevoeging van ZnO, CBS of zwavel onderdrukt de additiereactie tussen $3 \mathrm{~m} 1 \mathrm{p}$ en Si263. Inplaats daarvan werden hoge hoeveelheden disulfidesilanen (ook tri-sulfide silanen in het geval van zwavel adderende systemen) waargenomen als omzetting van Si 263. Dit geeft aan, dat de aanwezigheid van deze ingrediënten de gewenste reactie van mercapto-silaan verandert van de radicalaire additie reactie naat de koppelings reactie tussen twee silanen. Wanneer men deze kennis overdraagt op het rubber systeem, dan zou de toevoeging van deze ingrediënten kunnen leiden tot onderdrukking van de reactie tussen rubber en mercapto-silaan. Indien de reden voor de slechtere verwerkbaarheid van het mercapto-silaan systeem gelegen is in de overmatige reactie tussen het mercapto-silaan en de rubber, dan zouden deze ingrediënten de mogelijkheid hiervan kunnen verhelpen.

Daarna werd de modelstudie veranderd door gebruikmaking van squaleen of vloeibare butadiëen-rubber als model-stoffen: Hoofdstuk 5. Omdat die meer dan één dubbele binding hebben in een molecuul en een hoger moelcuul-gewicht, zouden de systemen de verschijnselen in het feitelijke banden loopvlak-mengsel nauwkeuriger moeten simuleren in vergelijking met het geval waarin model-olefinen worden gebruikt. De waargenomen verschuivingen zijn feitelijk in goede overeenstemming met de resultaten van het olefinenonderzoek. De waargenomen mate van isomerisatie in de Si 263 systemen was hoger dan in de Si 69 systemen. Bovendien werd het additie-product van Si 263 aan de vinyl en cis dubbele bindingen in vloeibaar butadiëen rubber en aan squaleen waargenomen, ofschoon de hoeveelheid in het squaleen systeem aanzienlijk lager was. In het Si 69 systeem was de hoeveelheid isomerisatie laag en mogelijke additie-producten werden niet waargenomen. Echter, een afname van de hoeveelheid dubbele bindingen werd waargenomen in alle Si 69 systemen. Omdat het 13C-NMR spectrum in het squaleen/Si 69 monster na verhitting een sterk vergelijkbaar signaal-patroon vertoont als degene waargenomen in het squaleen/zwavel systeem zonder enige versnellers na verhitting, werd de vorming van vernette producten ook in het Si 69 systeem sterk aangeduid. Dit correspondeert met het verkregen resultaat in het model olefine TME-systeem. Daarom kan worden geconcludeerd, dat Si 69 een rol heeft gespeeld als zwavel donor zonder additioneel zwavel of versnellers, zoals al aangegeven in vele wetenschappelijke artikelen.

Alle resultaten van de model studie werden nu gebruikt als een goede basis voor verdere onderzoeken in het rubber mengsel. In Hoofdstuk 6 werd het mengen van het typische passagiers-auto bandenloopvlak-mengsel uitgevoerd. Dit bevatte een SBR/BR mengsel met 
silica in combinatie met een mercapto-silaan of een sulfide-silaan. De verkregen interne structuur en mengsel eigenschappen werden verklaard op basis van de waarnemingen in de model-studie. De Si 263 mengsels vormen grotere hoeveelheden chemisch gebonden rubber, zelfs bij kortere mengtijd,vergeleken met de Si 69 mengsels. Bovendien werd de chemisch gebonden rubber in het Si 263 mengsel bepaald door zwellingsmetingen en gaf een aanzienlijk dichter polymeer-netwerk aan. Zoals al waargenomen in de model-studie verhoogt de zeer hoge reactiviteit van het mercapto-silaan radicaal aan de rubber-polymere dubbele bindingen, speciaal aan vinyl en cis dubbele bindingen, het aantal koppelingspunten tussen silica en rubber via silaan. Dit wordt ondersteund door de hoge radicalaire keten reactiviteit van SBR polymeer-radicalen gevormd door de omkeerbare additie-reactie en de abstractie van het allylische waterstof door de mercapto-silaan radicalen en de intermediaire (thioether en koolstof-gecentreerde) radicalen. De voortzetting van de radicalaire keten-reactie leidt tot de vorming van grote hoeveelheden $\mathrm{C}-\mathrm{C}$ bindingen resulterend in een dicht polymeer netwerk.

De Si 263 mengsels leverden bij gelijke mengtijd lagere tan $\delta$ bij $60^{\circ} \mathrm{C}$ vergeleken met de Si 69 mengsels. Bovendien schijnen de waardes te correleren met de Payne effect waardes van de gevulkaniseerde mengsels. Door de flocculatiemetingen aan de onvernette mengsels werd bevestigd, dat de Si 263 mengsels minder flocculeren, hetgeen resulteert in lagere Payne effect waardes evenals lagere tan $\delta$ waardes bij $60^{\circ} \mathrm{C}$ na vulkanisatie. Dit verschil lijkt terug te voeren op de hoge hoeveelheid chemisch gebonden rubber samen met de dichte en ontwikkelde structuur in de mercapto-silaan mengsels. De gebonden rubber schermt het silica oppervlak af en bovendien vermindert de gevormde dichte en ontwikkelde structuur de re-agglomeratie neiging van silica-clusters. De onderdrukking van de silica-flocculatie werd het duidelijkst waargenomen in het hoog-vinyl type SBR/Si 263 systeem. Dit schijnt terug te voeren op de hoogste hoeveelheid bindings-punten tussen silica en polymeer via silaan. Echter, in alle SBR/Si 263 systemen werd verhoging van de Mooney viscositeit of neiging tot verslechtering van het uitzien van het geloste mengsel uit de menging waargenomen met toenemende mengtijd. Dit geeft aan, dat de hogere hoeveelheden chemische bindingen tussen polymeer en silica via de silaan, in combinatie met de ontwikkelde dichte gebonden rubber netwerken, een lagere flexibiliteit veroorzaken van de ongevulkaniseerde mengsels, hetgeen tot de waargenomen slechtere verwerkbaarheid leidt. 
Als geheel genomen schijnt een toenemende directe binding tussen silica en polymeer via silaan, teneinde een voldoende afschermend effect te bereiken door polymeer op het silica oppervlak, tezamen met het voorkomen van een to dicht gebonden rubber netwerk veroorzaakt door een overmatige hoeveelheid $\mathrm{C}-\mathrm{C}$ binding, de betere richting te zijn om silica dispersie en verwerkbaarheid in balans te brengen.

In Hoofdstuk 7 werd het mengen van silica-gevulde NR of IR mengsels uitgevoerd. De resultaten werden vergeleken met die van Hoofdstuk 6, waarin SBR/BR mengsels zijn gebruikt. Ofschoon een hoger chemisch gebonden rubber gehalte werd verkregen in de $\mathrm{Si}$ 263 mengsels, wat dezelfde waarneming vertegenwoordigt als in het SBR/BR systeem, was de maximum hoeveelheid gebonden rubber gehalte in de NR of IR/silaan mengsels beperkt tot hetzelfde niveau als dat in de SBR-BR/Si 69 mengsels en bereikte niet de maximum waarde verkregen in de SBR-BR/Si 263 mengsels. Het polymere netwerk van de chemisch gebonden rubber in de NR of IR/Si 263 mengsels vertoont een dichtere structuur als die van alle Si 69 mengsels met inbegrip van de SBR/BR mengsels, maar is minder dicht vergeleken met die van de SBR-BR/Si 263 mengsels. De lagere additie-reactiviteit van de mercaptosilaan radicalen aan de isopreen-type dubbele bindingen samen met de gewenste koppelings-reactie tussen tertiaire radicalen, die kunnen worden gevormd door allylische waterstof-abstractie en het daaropvolgende delocalisatie-effect, schijnen de voornaamste redenen te zijn voor het bereiken van een lager chemisch gebonden rubber gehalte vergeleken met de SBR systemen. Bovendien zou het gemak van breuk van de hoofdketen in de isopreen-type rubber ook de reden kunnen zijn voor het niet waarnemen van de drastische toename van het gebonden rubber gehalte met toenemende mengtijd.

De waardes van het Payne effect vóór en na temperen (annealing) bij $170{ }^{\circ} \mathrm{C}$ gaven aan dat de NR of IR/Si 263 mengsels een hogere silica flocculatie-onderdrukking vertonen vergeleken met de Si 69 mengsels, ofschoon de mate niet zo hoog was als voor de SBR$\mathrm{BR} / \mathrm{Si} 263$ mengsels. Aangezien er bijna geen verschil was in gebonden rubber gehalte tussen de silanen bij de langer gemengde mengsels, schijnt het hogere effect op de flocculatieonderdrukking gelegen in een lichtelijk dichter gebonden rubber netwerk samen met het afschermings-effect van het silica oppervlak, teweeg gebracht door de polymeren, die direct aan het silica gebonden zijn via silaan. An de ene kant is de mate van hindering van silica flocculatie niet zo hoog als in de SBR-BR/Si 263 mengsels, aan de andere kant werd de neiging tot Mooney viscositeits-verhoging met toenemende mengtijd niet waargenomen 
en schijnt dus de verwerkbaarheid van de mengsels beter te zijn dan in de SBR-BR/Si 263 mengsels.

Alles tezamen werd vastgesteld, dat de microstructuur van de polymeren de interne versterkende structuur in de mercapto-silaan systemen beïnvloedt. Het gebruik van mercapto-silaan samen met de isopreen-type polymeren leidde niet tot hetzelfde niveau van silica-dispersie na de vulcanisatie-stap als voor de butadiëen-type polymeren, ofschoon de verwerkbaarheid verbeterd leek. Bovendien kan de combinatie van de isopreen-type polymeren en silanen, die een grote hoeveelheid polymere radicalen zouden kunnen vormen zoals mercapto-silanen, niet de degradatie van het polymeer voorkomen. Verder is de langere mengtijd bij hogere temperatuur teneinde de silaniserings-reactie te verhogen, ook niet altijd wenselijk voor deze polymeren. Daarom wordt geconcludeerd, dar de ontwikkeling van een koppelings-agens nodig is, dat de silica en het polymeer kan combineren zonder degradatie te veroorzaken.

De uiteindelijke conclusies zijn dat, waar silica gevulde mengsel technologieën vandaag de dag de wereldwijde standaard zijn voor passagiers-auto banden loopvlak-mengsels, verdere vebeteringen van de functionailteit altijd gewenst blijven. Het verbeteren van het dispersie-niveau van hogere specifieke oppervlakte silica, wat algemeen moeilijker te dispergeren is in de rubber matrix, lijkt een van de positieve richtingen te zijn om het versterkingsniveau te verhogen ten behoeve van verlaging van de mogelijke energiedissipatie gedurende de omwenteling van de band. In het huidige proefschrift werd de versterkende structuur opgehelderd welke een lagere vulstof-vulstof interactie na vulcanisatie realiseert, door onderzoek naar het reactie-mechanisme van sulfide-silaan en mercapto-silaan. Voornamelijk twee punten schijnen de reden te zijn waardoor mercaptosilanen een lagere vulstof-vulstof interactie geven. De eerste is een hogere afschermende werking van een polymeer gehecht aan het silica-oppervlak, ten gevolge van het hogere vermogen van mercapto-silanen om te hechten aan de dubbele binding. Het tweede punt is de vorming van een dichte gebonden rubber structuur door de radicalaire propagatiereactie tussen polymeren, aangezet door het polymere koolstof-radicaal geïnduceerd door het mercapto-silaan radicaal. Deze structuren onderdrukken de silica flocculatie gedurende de vulcanisatie-stap, resulterend in de waargenomen lagere Payne effect waardes. Echter, aangezien de effectiviteit van de mercapto-silanen op de boven vermelde twee punten afhangt van de dubbele binding structuur van het polymeer, zijn de mercapto-silanen niet toepasbaar voor alle types polymeren. Bovendien kan het tweede punt vooral de 
waargenomen slechtere verwerkbaarheid verklaren door de aanwezigheid van het boven genoemde dichte polymeer netwerk, reeds in het ongevulcaniseerde mengsel. Verdere onderzoeken zijn nog nodig om de optimale wijze te vinden waarop het betere silica dispersie-niveau en de verwerkbaarheid kunnen worden uitgebalanceerd, ongeacht het polymeer type. Echter, de waarnemingen gepresenteerd in het onderhavige proefschrift geven vele cruciale aanwijzingen hoe gedurende de menging de reacties te controleren, die op moeten treden, en hoe ongewenste zij-reacties te onderdrukken. De controle zou kunnen worden bereikt door de structuur van de silanen en/of de polymeren te verbeteren, of door derde soort additieven die specifieke functies bezitten. De resultaten in dit proefschrift worden verwacht een waardevolle bijdrage te leveren teneinde de optimale controle te vinden, welke zou kunnen leiden tot volgende technische innovaties in de banden industrie. 


\section{Acknowledgements}

This project was carried out for three years under the contract between the University of Twente (UT) and the Yokohama Rubber Co., Ltd. Support from countless number of people has enabled me to carry out and achieve this project. I would like to express my appreciation to all of those who supported during this time.

First of all, I would like to express my sincere gratitude to my promotor and supervisor Prof. Dr. Anke Blume. I am not sure whether I could manage to complete this project without your constant motivation. You have always helped and encouraged me very kindly, I cannot thank you enough. I don't know how many times I asked you to cut out on sleeping time especially in the writing period. A lot of other students after me would also ask you, but, please take good care of yourself.

I want to extend my immense my gratitude to Prof. Dr. Jacques Noordermeer (Thank you very much for the Dutch summary), Assoc. Prof. Dr. Wilma Dierkes and Assist. Prof. W. kaewsakul for all the help, encouragement and insightful discussions. And, thank you, Ceciel ter Horst-Strootman, for all of your kind support. When I faced a problem with Dutch rules and regulations, you always tried to find a solution for me. Thanks to your organization, I could enjoy the stay in the Netherlands without worries.

I would like to extend my appreciation to the Yokohama Rubber Co., Ltd. for the financial support during this project. To Dr. Amino, I sincerely appreciate that you gave me the opportunity to do the PhD project and being a part of my graduation committee. To Dr. Mihara, your warm encouragements always helped me a lot to proceed with this project. Now when I finally reach this moment, I am feeling deep respect for you again that you completed your PhD project as the $1^{\text {st }} \mathrm{PhD}$ student from our company. I would like to extend my appreciation to members in the Amino Laboratory, Mr. Ashiura and members in the analytical development group in the Yokohama Rubber. Co., Ltd. Your support with experiments and various meaningful discussions were very informative for me to finish writing this thesis. To members in raw materials for compound group 1, I thank all of you for the support, encouragement and giving me the time to work for this project despite your busy schedules. I'm looking forward to working with you in the same group soon. 
I would like to appreciate and express my regards to Prof. Dr. U. Giese, Prof. Dr. M. S. Galimberti, Prof. Dr. A. J. A. Winnubst, Dr. M. A. Hempenius, Mr. A. Hasse for accepting to be a part of my graduation committee.

I would also like to express my gratitude to people in Evonik, Wesseling, for joining meetings with me and many valuable discussions. And also, I really appreciate $\mathrm{Mr}$. $\mathrm{H}$. Rahnenführer and Mr. K. Slickers for teaching me analytical measurement procedures.

To all of my colleagues in ETE group; André, Kumar, Nadia, Hans, Antonio, Jacob, Jin, Chesidi, Marzieh, Neven, Ayush, Indria, Dries, Gina, Marcel, Katarzyna, Chai, Rafal, Amir, Xiaozhen, Arqam, Zuzanna, Carmela, Anna, Łukasz, Sunkeun, Marcin and Priyanka. My encounter with all of you is my treasure. Thank you very much for your sincere friendship and cheerfulness that you all gave me all the time. My special thanks to Akansha and Fabian for being my paranymphs. Dear Akansha, you are the friend who I talked to the most when I stayed in Enschede and the one who has understood me the most. Arigatou for your support and kindness. When you worked in our company as an intern was also interesting and nice memory for me. Dear Fabian, I feel very lucky that we have done our PhD work almost in the same timing and could visit various places together. Your hometown, Würzburg, where you showed me around was such a beautiful place. Next time, I will show you Japan. And Chigusa-san, conversations with you in Japanese made me so relax when I was struggling with writing this thesis. Thank you very much.

To Japanese people who we could meet in Enschede; thanks to your support, we could manage to live in a foreign country and also enjoy this special period. Thank you very much.

Lastly to my family; my mother and parents-in-law, thanks to your help, we could manage to accomplish this project. Thank you very much. To my dearest son Keigo, your presence encouragede me and Saya always. I'm sorry for making you feel sad and asking you to be patient during the time that I had to live away from you in the Netherlands. Let me try to do my best to compensate for it in the days ahead. Dear Hayato, I'm so happy to be able to see you during this project. You always heal me a lot. When you grow up, I'd like to take you to the Netherlands and tell you our unforgettable memories there. Finally, Dear Saya, any words are not enough to express my thanks to you. I'm sorry for putting a lot of burden on you, and thank you always for understanding my situation and supporting me. Without your support, I could not have completed this project. The days in the Netherlands will remain as our precious memories. Let's come someday to Enschede again together. 
